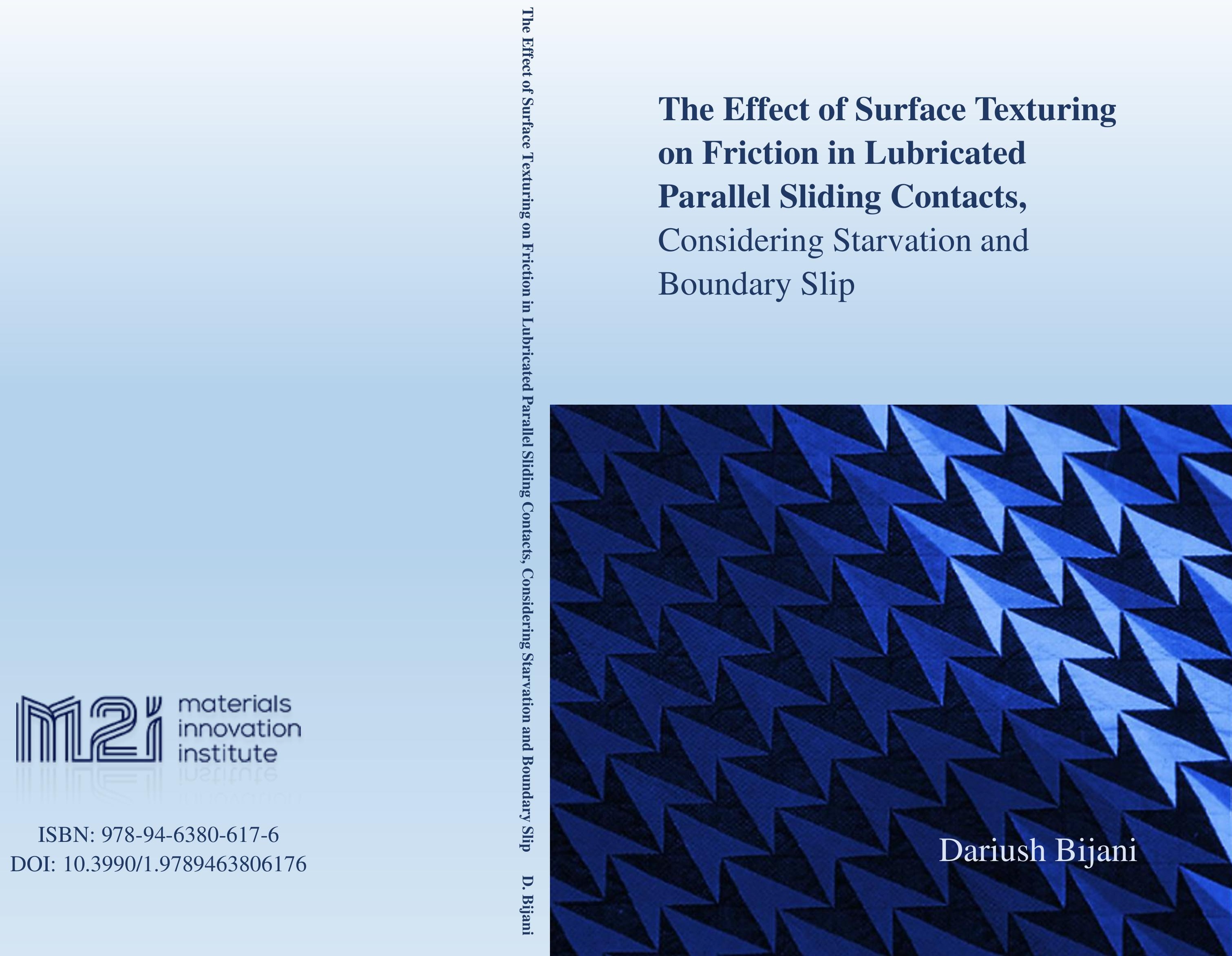




\section{THE EFFECT OF SURFACE TEXTURING ON FRICTION IN LUBRICATED PARALLEL SLIDING CONTACTS, CONSIDERING STARVATION AND BOUNDARY SLIP}

\section{Dariush Bijani}

\section{Faculty of Engineering Technology,}

Laboratory of Surface Technology and Tribology,

University of Twente 
This dissertation has been approved by Supervisor: Prof.dr.ir. D.J. Schipper Co-supervisor: Prof.dr.ir. M.B. de Rooij 


\title{
THE EFFECT OF SURFACE TEXTURING ON FRICTION IN LUBRICATED PARALLEL SLIDING CONTACTS, CONSIDERING STARVATION AND BOUNDARY SLIP
}

\section{DISSERTATION}

\author{
to obtain \\ the degree of doctor at the University of Twente, \\ on the authority of the rector magnificus, \\ Prof.dr. T.T.M. Palstra, \\ on account of the decision of the Doctorate Board, \\ to be publicly defended \\ on Friday 6th of December 2019 at 14.45 hours
}

By:

Dariush Bijani

born on 21st of September 1982

in Bojnord, Iran 
The research was carried out under project number M21.1.1148 in the framework of the research programme of the Materials innovation institute (M2i) in the Netherlands (www.m2i.nl).

De promotiecommissie is als volgt samengesteld:

Prof. dr. G.P.M.R. Dewulf

Prof. dr.ir. D.J. Schipper

Prof. dr.ir. M.B. de Rooij

Prof. dr.ir. J-P. Celis

Prof. dr. J.G.E. Gardeniers

Prof. dr.ir. G.B.R.E. Romer

Prof. dr. J. Sep
Universiteit Twente, voorzitter en secretaris

Universiteit Twente, promotor

Universiteit Twente, co-promotor

KU Leuven

Universiteit Twente

Universiteit Twente

Rzeszow University

Dariush Bijani

The Effect of Surface Texturing on Friction in Lubricated Parallel Sliding Contacts, Considering Starvation and Boundary Slip.

PhD Thesis, University of Twente, Enschede, the Netherlands, December, 2019

ISBN: 978-94-6380-617-6

DOI: $10.3990 / 1.9789463806176$

Copyright (C) 2019 Dariush Bijani, Enschede, the Netherlands, All rights reserved. No parts of this thesis may be reproduced, stored in a retrieval system or transmitted in any form or by any means without permission of the author.

Alle rechten voorbehouden. Niets uit deze uitgave mag worden vermenigvuldigd, in enige vorm of op enige wijze, zonder voorafgaande schriftelijke toestemming van de auteur. 
to Firouzeh and Mahmoud 


\section{Summary}

In practice, techniques are initiated to tune friction into the desired level in mechanically interacting surfaces. One of them is lubrication. However, in lubricated parallel sliding contacts without any geometrical features on the surface, no film thickness is generated and the two interacting surfaces will stick to each other and high friction occurs. Another challenge in lubricated contacts is that in industrial applications insufficient lubricant is supplied to contacts which results in a friction increase of the interacting components; this starved lubrication situation can cause significant damage in mechanical systems.

In industrial applications, when the operating conditions are fixed and the contact and lubricant characteristics are also fixed, the most important element to control the friction is the modification of interacting surfaces in contact. Over the past decades, surface engineers developed several methods to modify the physical and mechanical parameters of the surface techniques to have a better control on frictional behaviour. In parallel sliding contacts, by using surface texturing it is possible to create a lubricant film in contact and to trap wear debris, and cavities can act as oil reservoirs. In surface texturing, the geometrical properties of the texturing features can play an important role to minimize the friction in contact; therefore, investigating the influence of these properties is of interest. Another method to reduce friction is modifying the surface to create boundary slip.

In this thesis, the effect of surface texturing and boundary slip on lubricant film formation and friction is studied. The effect of different patterns on film thickness and different texturing properties, e.g. the size, depth and number of cavities and their distance from each other, is investigated numerically. To study the effect of texturing and boundary slip, the Reynolds equation is modified based on boundary conditions of a slippery surface. To consider the effect of cavitation, the Elrod cavitation algorithm is applied and the Reynolds equation is therefore modified.

In order to find the effect of texturing on friction, the mixed lubrication regime is studied. In the case of mixed and boundary lubrication due to the asperity contact, the solid on solid contact needs to be considered. Therefore, a deterministic asperity contact model is employed. By combining this approach with the film thickness algorithm, the coefficient of friction for different lubrication regimes is determined. Another important element that dominates the frictional behaviour of contacts is the amount of lubricant supplied to the contacts. In this study, the film thickness and friction for starved lubricated contacts are studied as a function of different texturing parameters.

To validate the results from the numerical study, several experimental measurements are performed. The challenge in the experimental study of parallel sliding contacts is making a perfect flat on flat contact, since the existence of even a micro-scale misalignment results in film formation that is not due to surface texturing. Therefore, a setup is designed to modify the tribometer to encounter misalignments. By employing the new setup, several experiments are performed and the results showed a close agreement between the experimental measurements and numerical results. 


\section{Samenvatting}

In de praktijk worden er verschillende technieken toegepast om wrijving af te stellen op het verwachte niveau wanneer verschillende oppervlakken mechanisch interageren. Eén van deze technieken is lubricatie. Echter, als de oppervlakken geen oneffenheden vertonen, wordt er geen film gecreëerd, waardoor de oppervlakken aan elkaar zullen kleven en er een hoge wrijving ontstaat. Een andere uitdaging in gelubriceerde contacten is dat er regelmatig onvoldoende lubricant gebruikt wordt, wat resulteert in een stijging van de wrijving, welke significante schade aan de mechanische onderdelen kan toebrengen. In industriële toepassingen, waar omstandigheden vast staan, net zoals de karakteristieken van de lubiricant en het contact, zij de oppervlakken die elkaar raken de belangrijkste factor die de wrijving beïnvloedt. De laatste tientallen jaren hebben oppervlakte ingenieurs verschillende methodes ontwikkeld die fysische en mechanische parameters kunnen aanpassen van de oppervlakken om zo een beter controle te krijgen over hun wrijvingsgedrag. Bij parallel glijden kan men door het gebruik van surface texturing er voor zorgen dat er wel een film ontstaan, waar vuil gevangen wordt in de openingen, welke ook optreden als olie reservoir. Bij dit surface texturing zijn de geometrische eigenschappen van de aangebrachte textuur van groot belang bij het minimaliseren van de wrijving; dit maakt het onderzoeken van deze eigenschappen erg interessant. Een andere methode om wrijving te minimaliseren is het aanpassen van het oppervlak zodat er boundary slip ontstaat.

In deze thesis wordt het effect van surface texturing en boundary slip op het vormen van een lubricatie film en wrijving onderzocht. Het effect van verschillende patronen op de dikte van de film en verschillende eigenschappen van de textuur, e.g. de diepte, grootte en aantal caviteiten en hun onderlinge afstand worden numeriek onderzocht. Om deze relatie te onderzoeken, bekijken we de Reynolds vergelijking en passen deze aan aan de randvoorwaarden van het oppervlak. Om cavitatie te onderzoeken, wordt het Elrod cavitatie algoritme gebruikt en maken we aanpassingen aan de Reynolds vergelijking.

On het effect van texturing op de wrijving te onderzoeken, wordt het mixed lubrication regime bestudeerd. Vanwege het asperity contact is het nodig bij mixed en boundary lubrication altijd het contact in rekening te nemen. Om deze reden gebruiken we een asparity contact model. Door het combineren van deze aanpak met het film thickness algoritme wordt de wrijving voor verschillende lubricatie methoden bepaald. Een ander aspect dat een grote invloed heeft of het wrijvingsgedrag van de contacten is hoeveelheid lubricant die gebruikt wordt. In deze studie wordt de film thickness wn wrijving onderzocht in situaties met lubricant armoede, bij verschillende textuurparameters.

Om de resultaten van de numerieke studie te valideren worden er verschillende experimentele metingen uitgevoerd. De uitdaging van deze experimentele studie van parallel glijdende contacten is het creëeren van een perfect glad contact, aangezien het bestaan van zelfs een micro oneffenheid ervoor zorgt dat er een film gecreëerd word, ongerelateerd aan surface texturing. Hiertoe wordt een opstelling gedefinieerd waarbij de tribometer kan aangepast worden aan deze oneffenheden. Door deze nieuwe opstelling te gebruiken, worden er verschillende nieuwe experimenten uitgevoerd. De resultaten tonen een dichte samenhang tussen de experimentele metingen en de numerieke resultaten. 


\section{Acknowledgements}

I would like to thank those who made this thesis possible. My deepest appreciation to my promoter, Prof.dr.ir. D.J. Schipper. Dik, thank you for your patience and your support in completing this PhD study. Your guidance helped me throughout this research and writing of this thesis. Moreover, I would like to thank Prof.dr.ir. Matthijn de Rooij for his useful hint and insightful comments.

During this research, I had the honour to work with Piet Lugt, Rob Bosman, Marc van Drogen and Aydar Akchurin. I would like to thank you all for your support during our monthly meetings. Moreover, I would like to express my gratitude to Loredana Deladi for all her support and friendship during this research. I would like to thank Erik, Walter for their assistance during the experimental works. The project funder M2i and partners Bosch Transmission Technology and SKF are gratefully acknowledged. To my colleagues with whom I had a nice time in the university of Twente: Martijn, Matthijs, Febin, Agnieshka, Yibo, Adeel, Adriana, Dinesh, Jincan, Belinda, Debbie, Emile, Sheng and Mohammad thanks to all of you.

I wish to thank my parents, Firouzeh and Mahmoud and my lovely sister Oldouz for the support and love they gave me through every moment of my life; you are the most amazing. My dearest Parisa, thank you for all the colour, beauty that you brought to my life and thanks for the support and the encouragement that you gave me, I am happy someone like you exist.

Mohsen, Danial, Ardavan, Dina, Atefeh, Shayan, Komal, Milad, Niki, Damoon, Saghar and my joyful friend Wasabi I am blessed with your friendship, I want to thank you for all your support, the fun and joy that your friendship gave me. I appreciate your care and how you are passionate to help. Nilgoon and Hadi, I am lucky to have you as my friends for quite a long time, I want to thank you for your support and help during this period of my life.

I also want to thank my friends in Bright Society, Leon, Stijn, Marcel and Rita for their trust, help and support during the past three years, I wish you the best and success for the society that you build. 


\section{Contents}

\section{Part I}

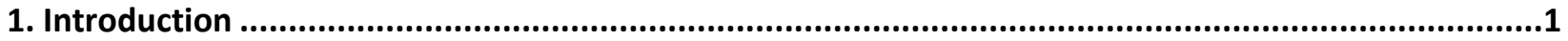

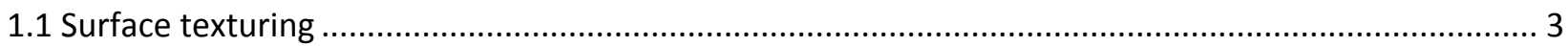

1.1.1 Parameters affecting the performance of surface texturing ….............................................. 3

1.1.1.1 Cavity shape and size effect on lubrication ....................................................................... 3

1.1.1.2 Effect of texture density on lubrication …......................................................................... 3

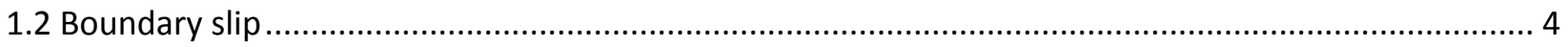

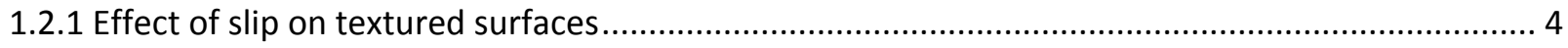

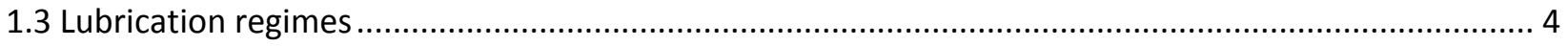

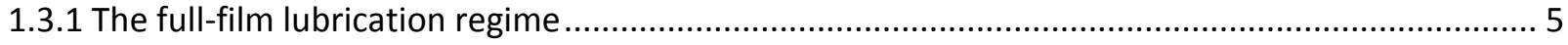

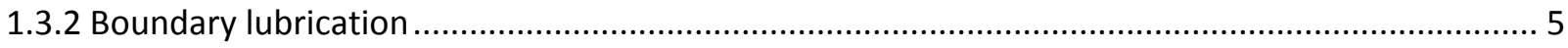

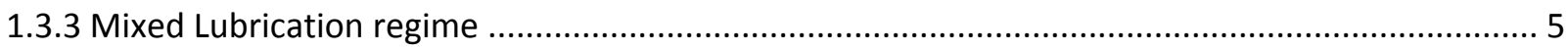

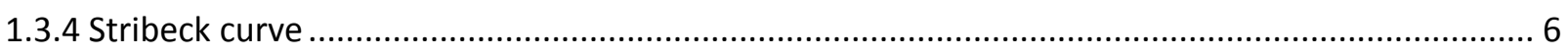

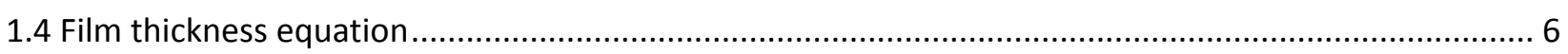

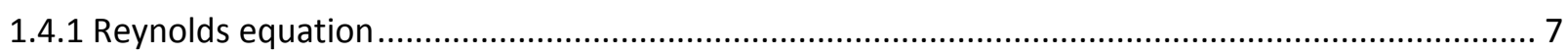

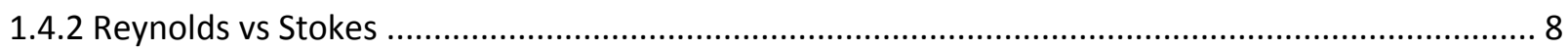

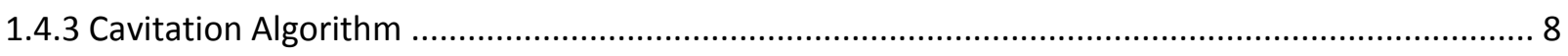

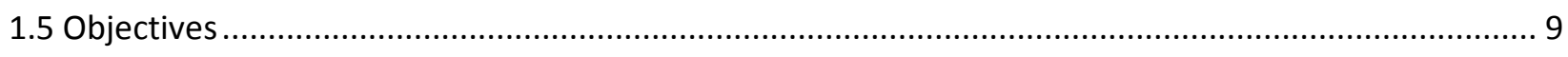

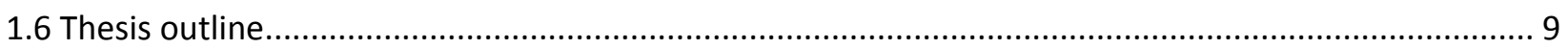

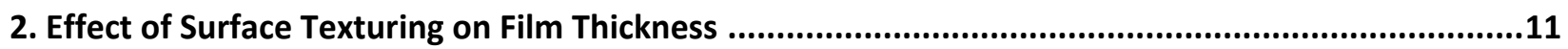

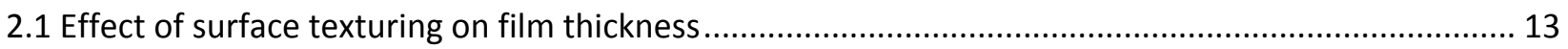

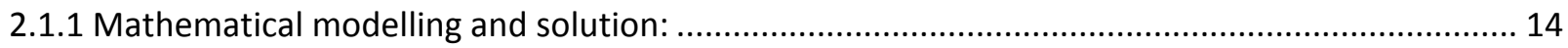

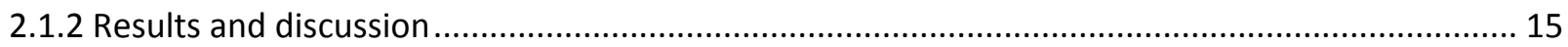

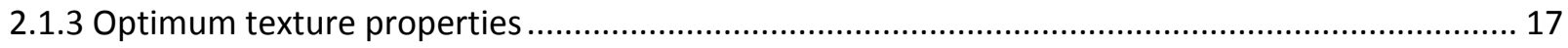

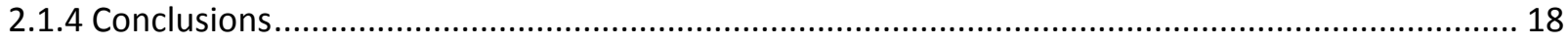

2.2 Surface texturing on film thickness in starved lubricated contacts ................................................ 19

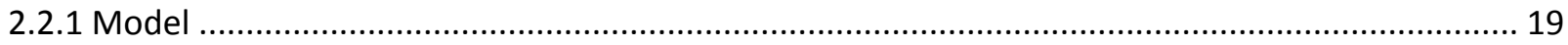

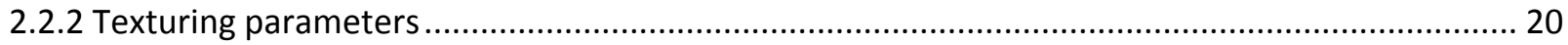

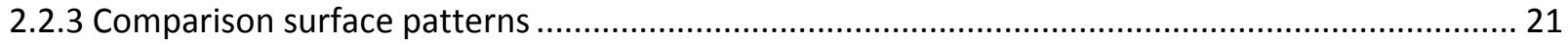

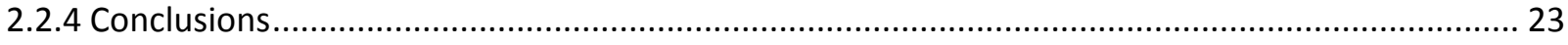

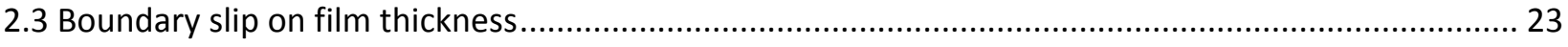

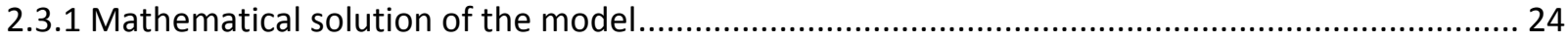




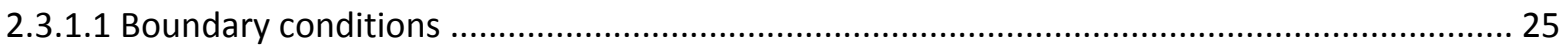

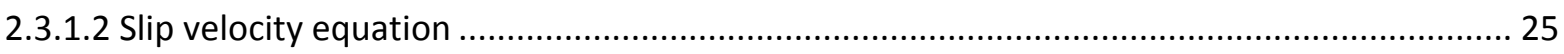

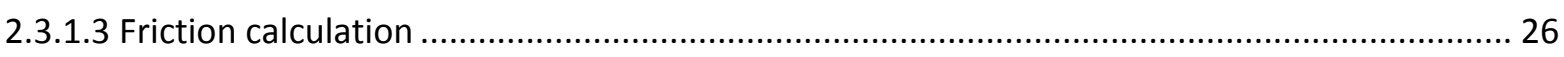

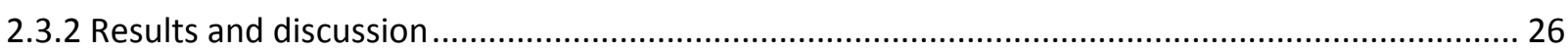

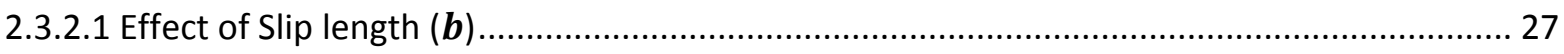

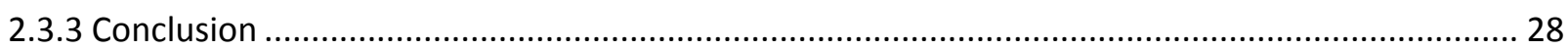

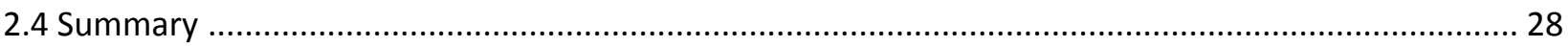

3. The Effect of Surface texturing on frictional behaviour of Sliding Contacts...................................29

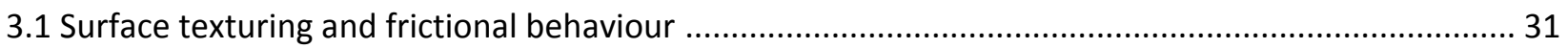

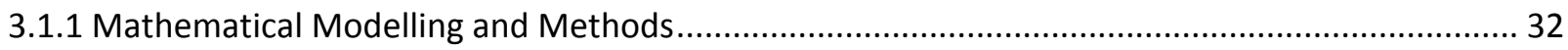

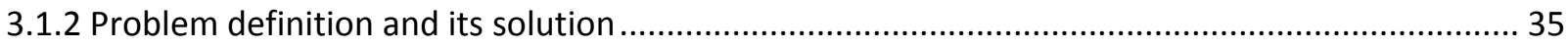

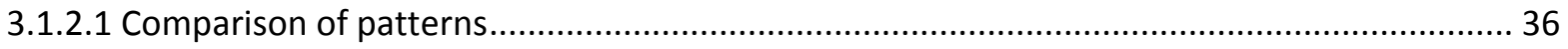

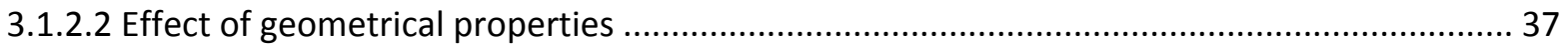

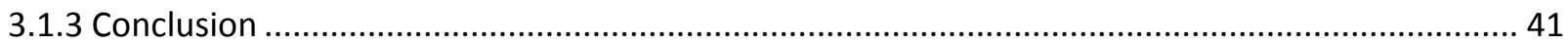

3.2 Surface texturing on frictional behaviour under starved lubrication condition ................................ 41

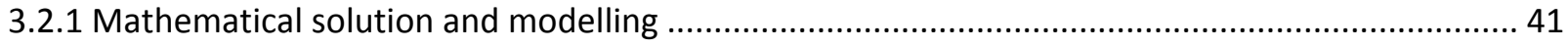

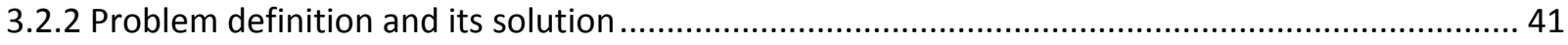

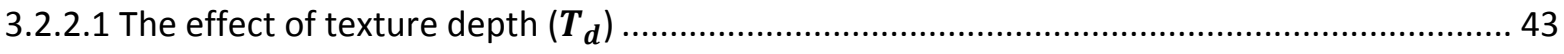

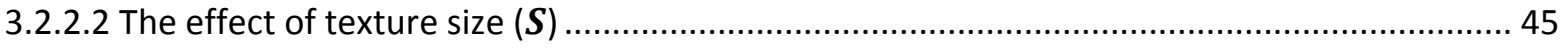

3.2.2.3 Effect of input film thickness on coefficient of friction ................................................... 47

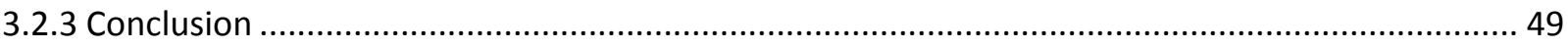

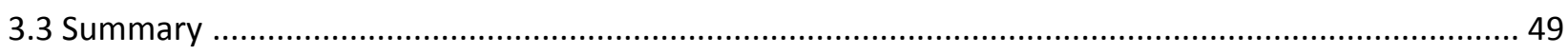

4. Experimental Validation of the Mixed Lubrication Model ............................................................51

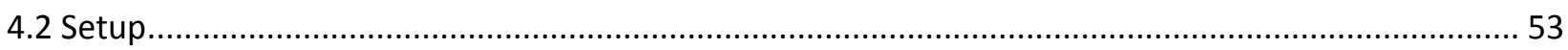

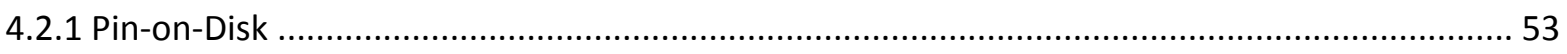

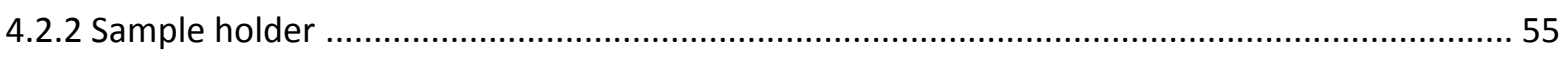

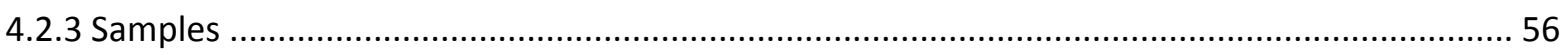

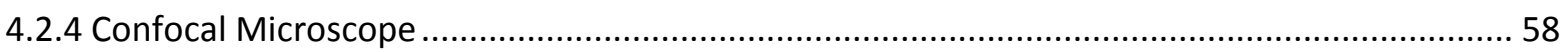

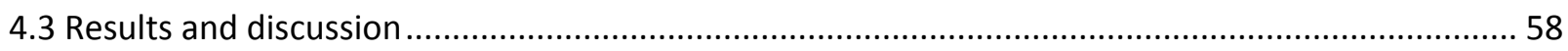

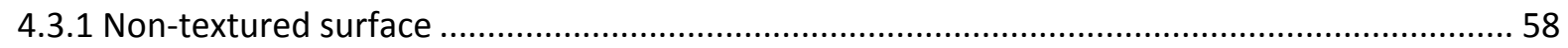

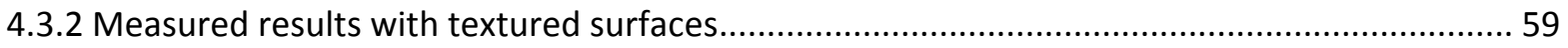

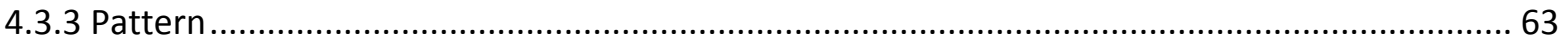

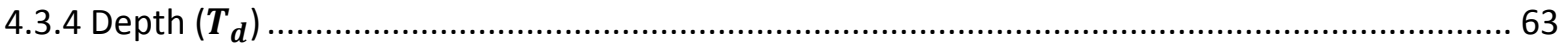

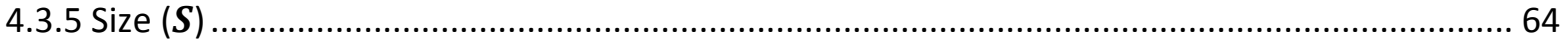

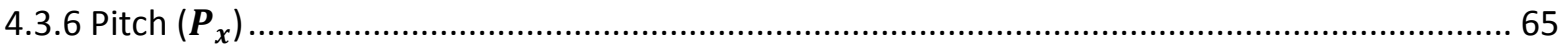




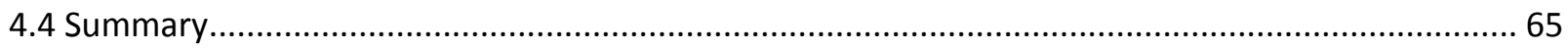

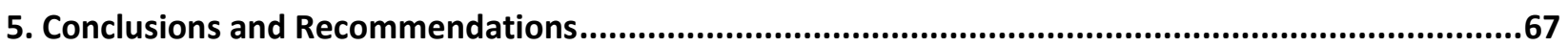

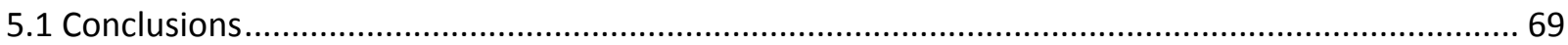

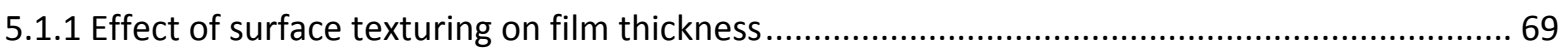

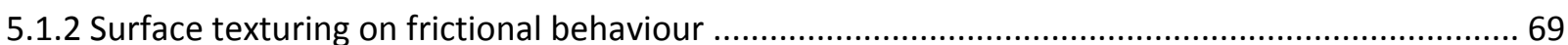

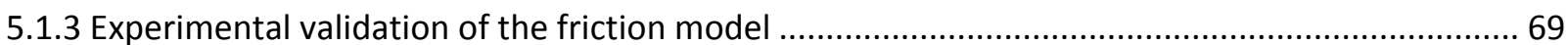

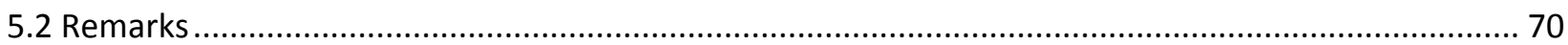

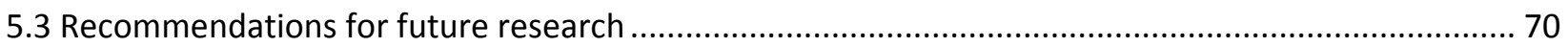

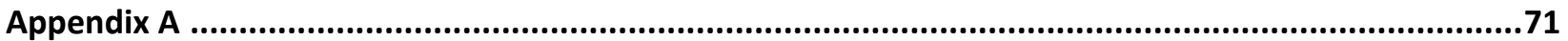

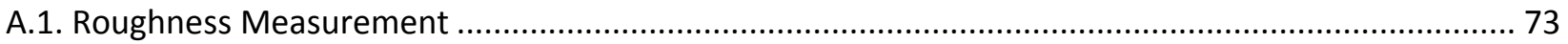

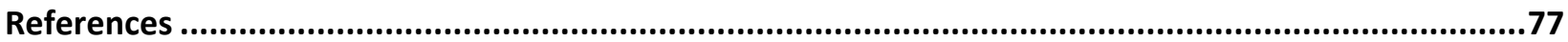

\section{Part II}

Paper A: The Influence of Surface Texturing on the Film Thickness in Parallel Sliding Surfaces.

Paper B: The Influence of Surface Texturing on the Film Thickness in Starved Lubricated Parallel Sliding Contacts.

Paper C: The Influence of Surface Texturing on the Frictional Behaviour of Parallel Sliding Lubricated Surfaces under Conditions of Mixed Lubrication.

Paper D: The influence of surface texturing on the frictional behaviour in starved lubricated parallel sliding contacts.

Paper E: The influence of surface texturing and boundary slip on the film thickness in parallel sliding surfaces.

Paper F: An experimental study on the influence of surface texturing in flat on flat lubricated contacts. 



\section{Nomenclature}

\begin{tabular}{|c|c|c|}
\hline Parameters & Description & Unit \\
\hline$\overline{A_{c}}$ & Real area of asperity contact & $m^{2}$ \\
\hline$a_{i}$ & Fit parameter $(i=1,2,3,4)$ & - \\
\hline$b$ & Slip length & $m$ \\
\hline $\mathrm{d}$ & Separation & $m$ \\
\hline E & Elasticity modulus & $\mathrm{GPa}$ \\
\hline$F$ & Elrod cavitation algorithm switch function & - \\
\hline$F_{C}$ & Load carried by the asperities & $N$ \\
\hline$F_{H}$ & Load carried by the hydrodynamic component & $N$ \\
\hline$F_{T}$ & Normal load on the contact & $N$ \\
\hline$F_{3 / 2}$ & Integral identity & - \\
\hline$F_{f}$ & Friction force & $N$ \\
\hline$F_{f H}$ & Hydrodynamic friction force & $N$ \\
\hline G & Geometric parameter & - \\
\hline$h$ & Film thickness & $m$ \\
\hline$h_{0}$ & Contact separation & $m$ \\
\hline$H$ & Dimensionless local depth of textured surface & - \\
\hline$h_{o i l}$ & Limited value of input film thickness & $m$ \\
\hline$L_{S}$ & Slip area length & $m$ \\
\hline$L_{x}$ & Textured area in $\mathrm{x}$-direction & $m$ \\
\hline$L_{y}$ & Textured area in y-direction & $m$ \\
\hline $\bar{n}$ & Density of asperities & - \\
\hline$P$ & Dimensionless pressure & - \\
\hline$p$ & Pressure & $P a$ \\
\hline$p_{a}$ & Ambient pressure & $P a$ \\
\hline$p_{C}$ & Cavitation pressure & $P a$ \\
\hline$p_{c}$ & Asperity pressure & $P a$ \\
\hline$p_{H}$ & Hydrodynamic pressure & $P a$ \\
\hline$p_{T}$ & Total pressure & $P a$ \\
\hline$r$ & Texture cell length in $x$ and $y$-direction, in the case of dimples & $m$ \\
\hline$r_{p}$ & Cavity characteristic width & $m$ \\
\hline$S$ & Cavity length $=2 r_{p}$ & $m$ \\
\hline$S_{p}$ & Textured area fraction & - \\
\hline$T_{d}$ & Texture depth & $m$ \\
\hline$u_{0}$ & Sum velocity & $m \cdot s^{-1}$ \\
\hline$u_{s}$ & Slip velocity in $\mathrm{x}$-direction & $m \cdot s^{-1}$ \\
\hline$v_{s}$ & Slip velocity in y-direction & $m \cdot s^{-1}$ \\
\hline$w_{i}$ & compliance of an asperity & $m$ \\
\hline$X$ & Dimensionless Cartesian coordination $=x / r_{p}$ & - \\
\hline$Y$ & Dimensionless Cartesian coordination $=y / r_{p}$ & - \\
\hline$X_{S}$ & Dimensionless Cartesian slip length & - \\
\hline$Y_{S}$ & Dimensionless Cartesian slip width & - \\
\hline$z_{i}$ & Asperity height & $m$ \\
\hline
\end{tabular}


Greek symbols:

\begin{tabular}{llc}
\hline Parameters & Description & Unit \\
\hline$\beta_{i}$ & Combined summit radius & $\mathrm{m}$ \\
$\dot{\gamma}$ & Shear rate & $\mathrm{S}^{-1}$ \\
$\eta$ & Dynamic viscosity & - \\
$\gamma_{1}$ & Adaption parameter for hydrodynamic component in $\mathrm{ML}$ & - \\
$\gamma_{2}$ & Adaption parameter for asperity contact component in $\mathrm{ML}$ & - \\
$\mu$ & Coefficient of friction & - \\
$\mu_{c}$ & Coefficient of friction in BL regime & - \\
$\mu_{r e f}$ & Coefficient of friction for reference condition & - \\
$\mu^{*}$ & Normalized coefficient of friction for slip length & - \\
$v$ & Poisson's ratio & - \\
$\xi$ & Cavity geometrical ratio & $\mathrm{kg} . \mathrm{m}^{-3}$ \\
$\rho$ & Lubricant density & $\mathrm{kg} . \mathrm{m}^{-3}$ \\
$\rho_{c}$ & Lubricant density in full film region & $\mathrm{m}$ \\
$\sigma_{s}$ & Standard deviation of the asperity height distribution & - \\
$\bar{\sigma}_{s}$ & Dimensionless roughness & $P a$ \\
$\tau_{C}$ & Shear stress at asperity contact & $P a$ \\
$\tau_{c o}$ & Critical shear stress & $P a$ \\
$\tau_{c x}$ & Shear stress x-component when slip takes place at solid surface & $P a$ \\
$\tau_{x z}$ & Shear stress & - \\
\hline
\end{tabular}


Part I 



\section{Chapter 1}

\section{Introduction}
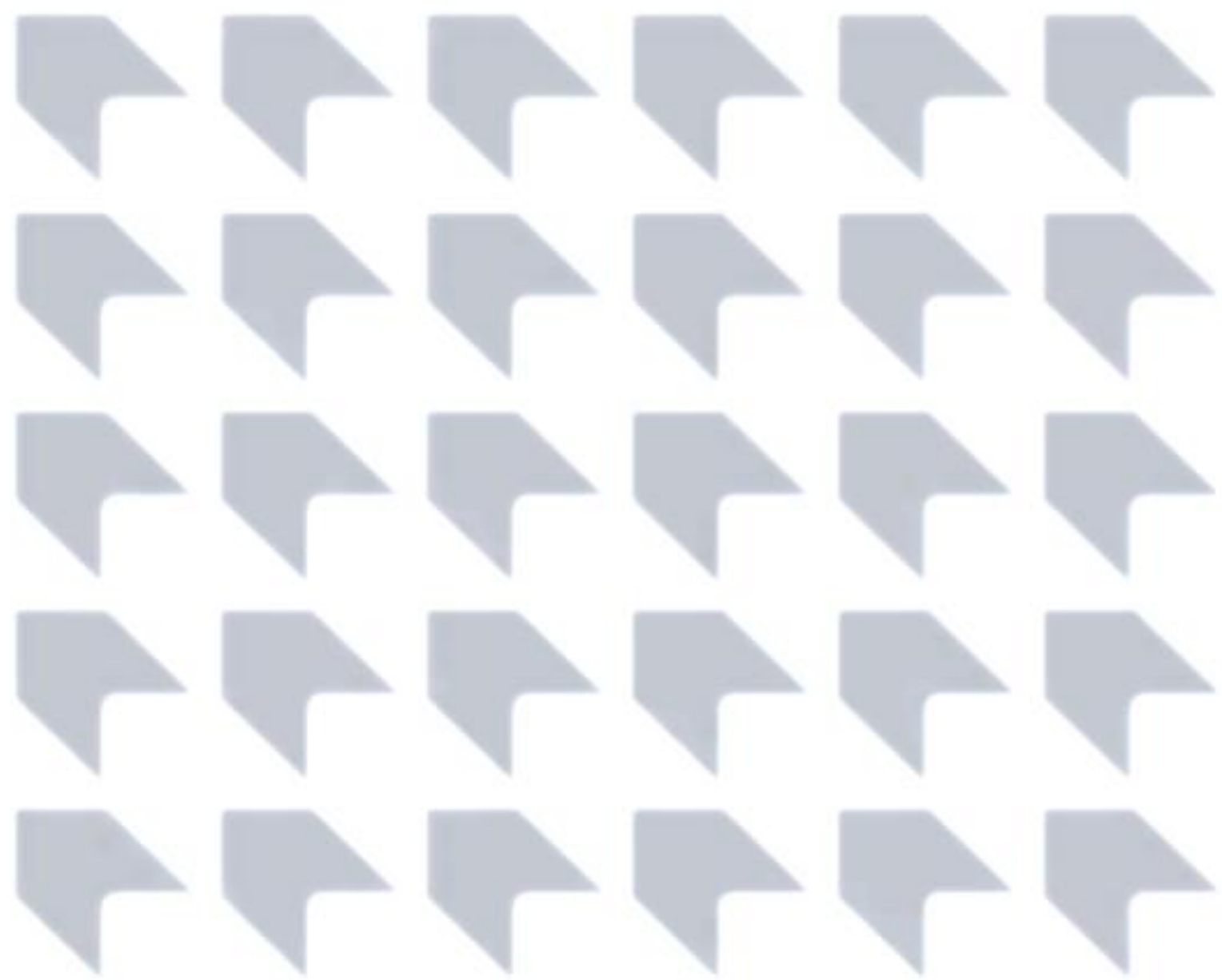


\section{Introduction}

Since the birth of civilization, it has been a challenge for man to use and control friction, a challenge that still continues. Friction is the force that resists the movement of objects. To control and minimize friction, it is common to introduce a lubricant between the contacting surfaces. In the automotive industry, for instance, the study of frictional behaviour and tribological performances of contacts plays a vital role in increasing efficiency and reducing fuel consumption. In order to decrease friction and wear between two contacting surfaces, it is common to separate the two surfaces with a lubricant film. In recent decades, thanks to improvements in modern industrial technology, new methods have been introduced to enhance and have more control over the frictional behaviour of contacts. This study focuses on two methods of achieving this goal: surface texturing and boundary slip. Furthermore, attention is paid to parameters that may have influence on friction by applying surface texturing and/or boundary slip.

\subsection{Surface texturing}

Surface texturing has emerged in the last decade as a reliable option in surface engineering, resulting in significant improvement in the load-carrying capacity, wear resistance and friction of tribological mechanical components. The cavities in textured areas may result in an improvement in tribological performance due to local converging and diverging gaps, generating pressure even in sliding flat-on-flat contacts. Three main effects are expected to occur to enhance the tribological performance of textured surfaces. The first effect is the improvement of hydrodynamic pressure in contacts due to the converging wedges constituted by the pockets [1]. The second is the absorbing of wear debris inside the cavities, which can be beneficial by removing the wear particles between the two surfaces, which could cause abrasive wear [2-4]. The third effect is the existence of pockets containing a lubricant which could be particularly useful in conditions involving high deformation processes [5].

Various techniques are available for surface texturing but Laser Surface Texturing (LST) is probably the most advanced so far. LST produces a very large number of micro-cavities in the surface and each of these micro-cavities can serve either as a micro-hydrodynamic bearing in cases of full or mixed lubrication, a micro-reservoir for lubricant in cases of starved lubrication, or a micro-trap for wear debris in either lubricated or dry sliding.

It is necessary to identify the effect of each geometry parameter that defines the texturing pattern. Therefore, it is important to study the most important parameters influencing lubricated contacts.

\subsubsection{Parameters affecting the performance of surface texturing}

\subsubsection{Cavity shape and size effect on lubrication}

Well-defined micro-cavities can act as lubricant reservoirs capable of feeding lubricant directly into the contact, initiating hydrodynamic lubrication as well as traps for wear debris.

Several studies showed that the beneficial effect of cavities becomes greater with an increase in depth [611]. The geometric shape and orientation of the cavity have obvious influence on the load-carrying capacity of contacting surfaces.

\subsubsection{Effect of texture density on lubrication}

Analysis showed that the textured area density is an important parameter in improving the frictional behaviour of surfaces [12-14]. Before passing the specific texture density (optimum value), increasing the texture density is more beneficial for lubrication regime transitions from mixed lubrication to hydrodynamic lubrication and after passing the optimum value of texture density, increasing the textured area density has a negative effect over the transition $[15,16]$. 


\subsection{Boundary slip}

Slip of a liquid at a solid wall is especially important to engineering contacts involving liquid-solid interfacial phenomena, such as lubrication, flows through porous media and liquid coatings. Many surfaces in nature are highly hydrophobic. The best-known example of a hydrophobic self-cleaning surface is the leaves of the lotus plant.

Although the classic fluid-dynamic assumption of a no-slip boundary condition (i.e. relative zero flow velocity at a solid wall) is quite satisfactory in dealing with most viscous flow problems of continuum fluids. Molecular dynamics simulations have shown that microscopic slip is possible, depending on several fluidsolid interfacial parameters. Boundary slippage allows the breakdown of the solid/liquid bonding such that the shear resisting force is reduced through the slip at the solid/liquid interface. Therefore, boundary slippage can be presented as an alternative for friction reduction, whereby the first layer of lubricant molecules moves with a different velocity from that of the adjacent solid surface. For the no-slip boundary condition, the first layer of fluid molecules has exactly the same velocity as its contacting solid surface and has been widely accepted in the field of fluid mechanics.

In the study of Tauviqirrahman et al. [17] the effect of boundary slip on friction reduction is investigated; they achieved an optimized complex slip surface and an optimized slope incline ratio simultaneously. It was shown that surface optimization of a parallel sliding gap with a slip surface can double the hydrodynamic load-carrying capacity and reduce the friction force by half of what the Reynolds theory predicts for an optimal slope inclination of a traditional slider contact. For a two-dimensional (finite length) journal bearing, Ma et al. [18] showed that the optimization of the shape and size of the surface may give advanced properties.

\subsubsection{Effect of slip on textured surfaces}

Work by Tauviqirrahman et al. [19] showed that like surface texturing, well-chosen slip/no-slip surface patterning can considerably improve the performance of fluid bearings. In their study, Tauviqirrahman et al. [20] investigated the combined effect of texturing and boundary slippage in lubricated sliding contacts. They confirmed that the textured surface employing boundary slip has a much lower coefficient of friction than a non-textured surface, as well as a solely textured surface. In their study, the load-carrying capacity is determined by integrating the calculated hydrodynamic pressure field along the contact surface. In their calculations, the stationary surface is designated as a textured surface and the entire contact is under the full fluid lubrication conditions (See Fig. 1.1).

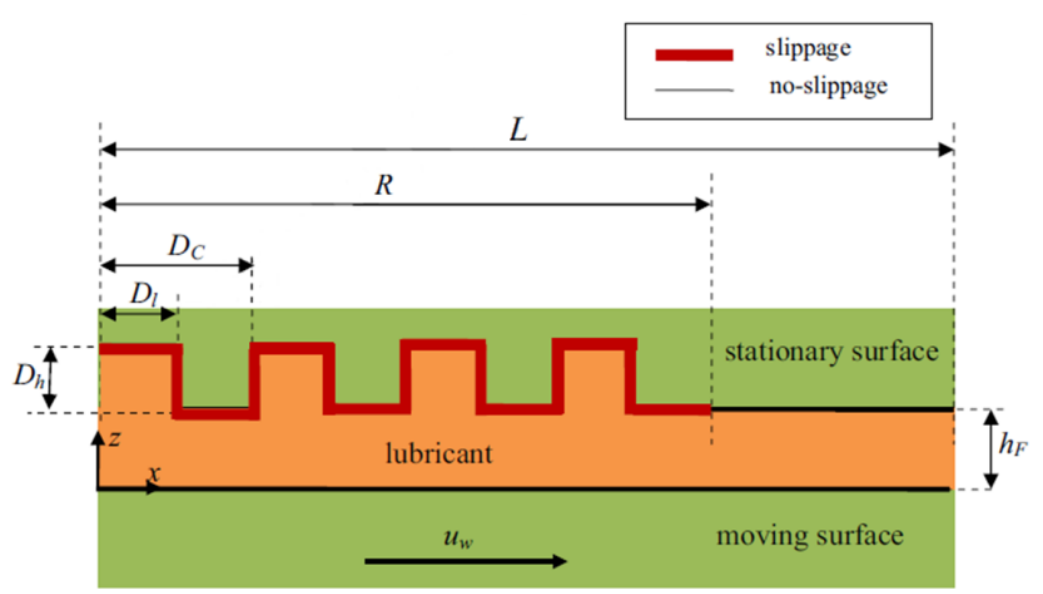

Figure 1.1. Schematic of a lubricated parallel sliding contact with a partially textured stationary surface combined with slippage at all sides of the texture cell [20].

\subsection{Lubrication regimes}


Depending on the application and the operating conditions it is common to characterize the tribological contact by its lubrication regime. The lubrication regimes are often divided into Boundary Lubrication (BL), Mixed Lubrication (ML) and Full Film Lubrication (FL). The three main states of lubrication are illustrated schematically in (Fig. 1.2).

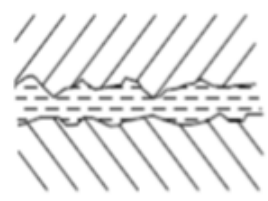

FL

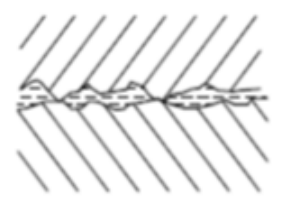

ML

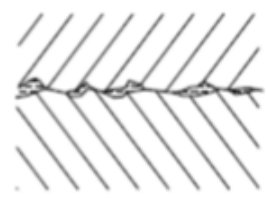

BL

Figure 1.2. Lubrication regimes.

\subsubsection{The full-film lubrication regime}

When the hydrodynamic action of the lubricant separates the surfaces fully and the load is carried solely by the lubricant film, the contact operates in the full film lubrication (FL) regime. In the FL regime, traction may be reduced by carefully chosen topographies. In the hydrodynamic lubrication regime the film is generally thick, so the opposing solid surfaces are prevented from coming into contact. This condition is often referred to as "the ideal form of lubrication" since it provides low friction and prevents wear. The lubrication of the solid surfaces is governed by the operating conditions, such as velocity, geometry and the bulk physical properties of the lubricant, notably the viscosity. The frictional characteristics arise purely from the shearing of the viscous lubricant. The minimum film thickness in hydrodynamically lubricated contacts is a function of the applied load $F_{N}$, velocity $U$, lubricant viscosity $\eta$, and geometry. The film thickness for a hydrodynamic lubricated contact is in general given by (Eq. 1.1):

$$
(h)_{H L} \propto G\left(\frac{\eta u_{0}}{p}\right)^{1 / 2}
$$

in which $\mathrm{G}$ is a geometry parameter depending on the shape of the lubricated contact, $\eta$ is the viscosity, $u_{0}$ the sum velocity and $p$ the average contact pressure.

\subsubsection{Boundary lubrication}

When the solid surfaces are so close together asperities come into contact and others are separated by a thin layer of lubricant (see Fig. 1.3), the friction and wear in boundary lubrication are determined predominantly by the interaction between the interacting asperities. In this case, the bulk flow properties of the liquid play little or no part in the friction and wear behaviour. Boundary lubrication usually occurs under high-load and/or low-velocity conditions.

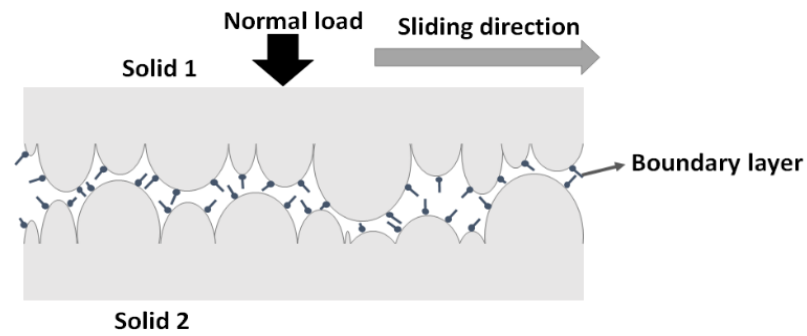

Figure 1.3. Schematic representation of two surfaces in contact.

\subsubsection{Mixed Lubrication regime}


The intermediate lubrication region between the $\mathrm{BL}$ and $\mathrm{HL}$ regimes is mixed lubrication, the load is transmitted partly by hydrodynamic pressure and partly by the contacting asperities. In the ML regime, the coefficient of friction ranges between $\mu_{H L}$ and $\mu_{B L}$. A very important parameter in lubricated tribosystems besides the operating conditions (load, velocity and temperature) and lubricant properties (i.e. viscosity) is the roughness of the surfaces, i.e. the micro geometrical irregularities of the surfaces. The roughness will influence the transition between the lubrication regimes when the surfaces carry the entire load by having direct contact (BL and ML regime).

Most machine components, such as gears and rolling bearings, are likely to operate in the mixed lubrication regime where significant asperity contacts exist. Modelling mixed lubrication is a challenging task; much effort has been devoted to this matter in recent decades. There are two types of mixed lubrication models: statistic and deterministic models. The statistic model uses selected statistic parameters to represent random characteristics of the surface roughness and concerns the averaged or statistical behaviour of the contact and lubrication. A major shortcoming of these models is their inability to provide detailed information about local affairs, which have a major influence on the mechanisms of lubrication and friction. The deterministic model, which uses deterministic information of surface roughness, is another approach to simulate the behaviour of contacting asperities in mixed lubrication.

The total normal load in ML regime is shared between the load carried by the contacting asperities and the load carried by the fluid film:

$$
F_{\mathrm{T}}=F_{\mathrm{C}}+F_{\mathrm{H}}
$$

where $F_{\mathrm{C}}$ is the load carried by asperities and $F_{\mathrm{H}}$ is the load carried by the hydrodynamic $(\mathrm{HL})$ component.

\subsubsection{Stribeck curve}

The coefficient of friction, i.e. the ratio between the friction force and the normal force of two moving surfaces, can be plotted for instance as a function of velocity using the Stribeck curve (see Fig. 1.4). The horizontal axis in (Fig. 1.4) has a logarithmic scale.

For high velocities $\mathrm{HL}$ takes place and for low velocities the $\mathrm{ML}$ and $\mathrm{BL}$ prevail. The increase in technical demands (small-sized components in combination with high loads) leads to a decrease in the film formation and therefore the contacts do not operate in the $\mathrm{HL}$ but in the $\mathrm{ML}$ or $\mathrm{BL}$ regime. Therefore the left hand side of the Stribeck curve is becoming more and more of interest. There are many factors that influence the friction curve, such as surface roughness, type of boundary layer, amount of oil supplied to the contact, etc.

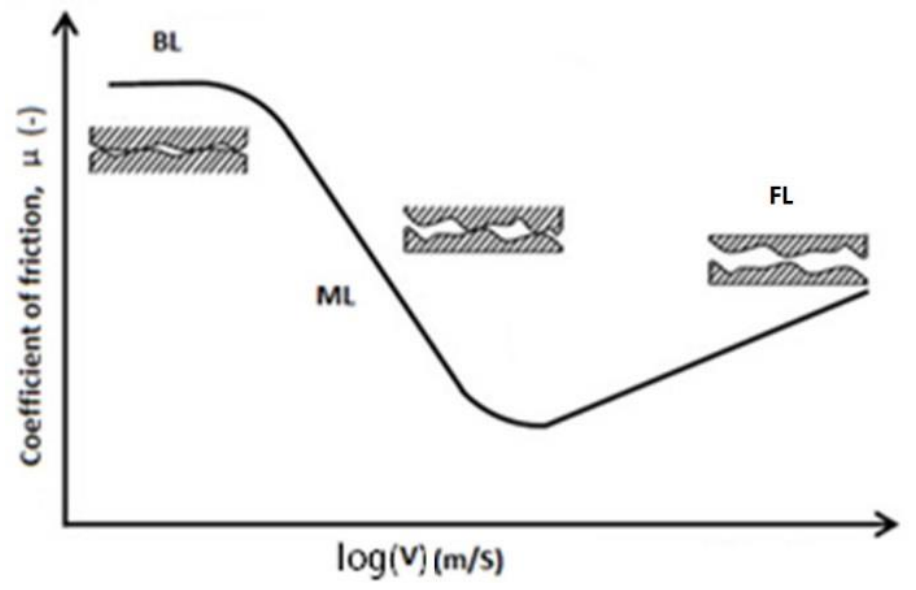

Figure 1.4. Schematic Stribeck curve.

\subsection{Film thickness equation}


The prediction of the film thickness under realistic conditions has been the focus of attention for the past five decades [21-25]. The Reynolds equation can be used to determine the pressure distribution in a lubricated contact. Some of the parameters affecting the film thickness are velocity, lubricant viscosity and contact geometry. All the aforementioned parameters have their own effect on the film thickness. Moreover, the study of starved lubrication has attracted the attention of tribologists for many years [2633]. This growing interest in the study of starvation has shown that, for example, most high-speed bearings operate under starved condition, i.e. when there is insufficient lubricant to fill the inlet of the conjunction.

\subsubsection{Reynolds equation}

The well-known Reynolds equation has been used for the past century to analyze all kinds of hydro/aerodynamic lubrication problems. In most cases, it accurately predicts the characteristics of the flow in the lubricant film. However, with decreasing film thickness in bearings, the roughness of the contacting surfaces becomes more important with respect to the flow and the Reynolds equation is less appropriate.

The Navier-Stokes (N-S) equations in Cartesian coordinates (Eq. 1.3) [5]:

$$
\frac{\partial(\rho v)}{\partial t}+\nabla \cdot(\rho \mathrm{v} \otimes \mathrm{v}+\mathrm{p} \overline{\mathrm{l}}-\bar{\tau})=\rho \mathrm{f}_{e}
$$

In addition, for $i, j=1,2,3$ and $\left(x^{1}, x^{2}, x^{3}\right)=(x, y, z) \in \Omega$ wehave:

$(\bar{l})_{i j}=\delta_{i j}$

$(\bar{\tau})_{i j}=\eta\left(\frac{\partial v^{j}}{\partial x^{i}}+\frac{\partial v^{i}}{\partial x^{j}}\right)-\frac{2}{3} \eta(\nabla \cdot v) \delta_{i j}$

$\mathrm{v}=(u, v, w)^{T}$ velocity vector

$f_{e}=$ external force field

Parameters like density and velocity have to satisfy the continuity (Eq. 1.4):

$$
\frac{\partial(\rho v)}{\partial t}+\nabla \cdot(\rho v)=0
$$

In 1886, Osborne Reynolds presented a partial differential equation to describe pressure build-up in selfacting bearings. This equation has been used successfully to determine the pressure distribution in the fluid film for a wide range of applications from bearings and seals to sheet metal forming processes. This equation is deduced from the Navier-Stokes equations. For defining the Reynolds equation, several assumptions considered:

1. Body forces are negligible.

2. The pressure is constant through the thickness of the film.

3. The curvature of surfaces is large compared with film thickness.

4. There is no slip at the boundaries.

5. The lubricant is Newtonian, i.e. stress is proportional to rate of shear.

6. The flow is laminar.

7. Fluid inertia is not considered.

8. The viscosity is constant through the film.

The Reynolds equation in Cartesian coordinates can be written as: 


$$
\frac{\partial}{\partial x}\left(\frac{\rho h^{3}}{\eta} \frac{\partial p}{\partial x}\right)+\frac{\partial}{\partial y}\left(\frac{\rho h^{3}}{\eta} \frac{\partial p}{\partial y}\right)=6\left(v^{+}\right) \frac{\partial(\rho h)}{\partial x}+6 \rho h \frac{\partial\left(v^{+}\right)}{\partial x}+12 \frac{\partial(\rho h)}{\partial t}
$$

with:

$$
\begin{array}{ll}
6\left(v^{+}\right) \frac{\partial(\rho h)}{\partial x} & \text { Wedge term, } \\
6 \rho h \frac{\partial\left(v^{+}\right)}{\partial x} & \text { Stretch term, } \\
12 \frac{\partial(\rho h)}{\partial t} & \text { Squeeze term, } \\
\frac{\partial}{\partial x}\left(\frac{\rho h^{3}}{\eta} \frac{\partial p}{\partial x}\right)+\frac{\partial}{\partial y}\left(\frac{\rho h^{3}}{\eta} \frac{\partial p}{\partial y}\right) & \text { Poisseuille terms. }
\end{array}
$$

Depending on the operating situation, several parts of the equation can be omitted.

\subsubsection{Reynolds vs Stokes}

For a Newtonian fluid, the Navier-Stokes equations describe the conservation of momentum. If the inertia terms are very small in comparison with the viscous terms, they reduce to the Stokes equations. Assuming that $h / L \ll 1$, where $h$ is the local film thickness and $L$ is a typical length scale in the contact, it follows from the Stokes equations that the pressure is independent of the cross-film coordinate. This simplifies the Stokes equations, and an expression for the velocity field as a function of the unknown pressure can be found. Substitution of the velocity field in the continuity equation and integrating it over the film thickness results in the Reynolds equation. Based on the anticipated validity of the Reynolds equation one now distinguishes two types of roughness:

Reynolds roughness versus Stokes roughness, see Elrod [25] and Van Odyck [34]. They are defined as:

- Reynolds roughness: $h / \Lambda_{C} \ll 1$,

- Stokes roughness: $h / \Lambda_{C} \gg 1$,

where $\Lambda_{C}$ represents the characteristic wavelength of the surface roughness. In the case of Reynolds roughness, the Reynolds equation appropriately describes the fluid flow in a lubricated contact, otherwise, the Stokes equations should be used.

In practice, assuming Reynolds roughness means assuming that the film geometry is such that both on a global and on a local scale its effect on film formation can be analyzed by means of the Reynolds equation. The validity of the Reynolds equation for textured surfaces is justified because the effect of texturing patterns and properties like depth and length is more dominant with respect to flow than the surface roughness, as a result the texturing pattern wavelength should be applied instead of the roughness characteristic wavelength $\Lambda_{C}$. If the length of the texture cavity is much greater than the film thickness, the Reynolds equation is valid. In a regular surface texturing, when the maximum cavity depth is in order of $10 \mu \mathrm{m}$ and the texturing wavelength is at least $100 \mu \mathrm{m}$ the ratio between texture depth and cavity length is usually less than 0.1 .

Based on work of Van Odyck [34] and Elrod [35] the narrow gap assumption and Reynolds equation are valid in the case of surface texturing in numerical modelling.

\subsubsection{Cavitation Algorithm}


At the outlet of the cavity, the lubricant is dragged through a converging region and, as a result, pressure is generated. At the entry of the cavity, the lubricant flow diverges, which results according to the Reynolds equation (Eq. 1.5) in a negative pressure. However, negative pressure is suppressed by cavitation at which vapour bubbles are formed in the lubricant.

For moderately loaded lubricated systems, the Jakobsson-Floberg-Olsson [36, 37] cavitation theory is used. It is not suitable for cases where surface tension plays an important role, such as in face seals [38]. Based on Jakobsson-Floberg-Olsson, the lubrication film is divided into two zones. The first part is with a complete lubricant film, in which the pressure varies; in this region the Reynolds equation applies. In the second part, cavitation happens and only a fraction of the lubricant film gap is occupied. Because of the intervening gas within the void fraction, the pressure throughout the cavitation area is taken as constant, [38].

According to the Payvar-Salant model $[25,39]$, the steady-state mass-conservation Reynolds equation, which takes cavitation into account, can be written in a Cartesian coordinate system as (Eq. 1.6) [40]:

$$
\frac{\partial}{\partial x}\left(\frac{h^{3}}{\eta} \frac{\partial(F \varphi)}{\partial x}\right)+\frac{\partial}{\partial x}\left(\frac{h^{3}}{\eta} \frac{\partial(F \varphi)}{\partial y}\right)=\frac{6 u_{0}}{p_{a}-p_{C}} \frac{\partial((1+(1-F) \varphi) h)}{\partial x}
$$

In this equation $h$ is the film thickness, $\eta$ is the viscosity, $u_{0}$ the sum velocity, $p_{a}$ is the ambient pressure, $p_{C}$ is the cavitation pressure, $\varphi$ is a dimensionless dependent variable and $F$ is the cavitation index. For more information the reader is referred to Wang [40].

Dobrica et al. [41] performed an investigation based on the influence of cavitation and different texturing parameters on the hydrodynamic performance of textured contacts. Their results are derived numerically, based on the Reynolds equation with the JFO formulation on the lubricated plane-parallel sliding contact and the lubricated sliding inclined contact. They studied the optimal textured region and the optimal dimple aspect ratio. In their investigation, cavitation was shown to have a significant influence on the performance of plane-parallel textured sliding contacts. In partially textured parallel sliders, cavitation had a positive effect by increasing the inlet flow (inlet suction). In convergent sliders with a high incline ratio, texturing showed a minimal effect as well as cavitation. In their study the optimal dimple depth and dimple length were determined for different plane-inclined sliders.

\subsection{Objectives}

Improving the frictional behaviour of contacts by applying the texture over the surfaces requires an indepth study of surface texturing effects and an optimization of texturing properties. In this study, the aim is to develop a theoretical friction model, which can be used to predict and reduce the coefficient of friction in lubricated plane-parallel sliding contacts. Based on this, it will provide innovative solutions for related applications and future designs.

Furthermore, the effect of starvation and boundary slip on film thickness and friction in the case of textured surfaces will be part of the analysis.

\subsection{Thesis outline}

This thesis is divided in two parts: the first part (I) of the thesis summarizes the study of surface texturing with respect to film thickness and friction (mixed lubrication), taking into account cavitation and boundary slip. In this part, an overview of formulation and the model is presented. In the second part (II) of the thesis, the publications based on different topics are presented.

In part I of this thesis:

- Chapter 2, discusses the effect of surface modification (texturing) and boundary slip on the lubricant film thickness. 
- Chapter 3, deals with the effect of surface texturing on friction.

- Chapter 4, introduces an experimental method to measure the coefficient of friction in parallel sliding contacts. Moreover, the validity of the mixed lubrication model is examined.

Part II (Papers):

A. The influence of surface texturing on the film thickness in parallel sliding surfaces.

B. The influence of surface texturing on the film thickness in starved lubricated parallel sliding contacts.

C. The influence of surface texturing on the frictional behaviour of parallel sliding lubricated surfaces under conditions of mixed lubrication.

D. The influence of surface texturing on the frictional behaviour in starved lubricated parallel sliding contacts.

E. The influence of surface texturing and boundary slip on the film thickness in parallel sliding surfaces.

F. An experimental study of the influence of surface texturing in flat on flat lubricated contacts. 


\section{Chapter 2}

Effect of Surface Texturing on Film

Thickness

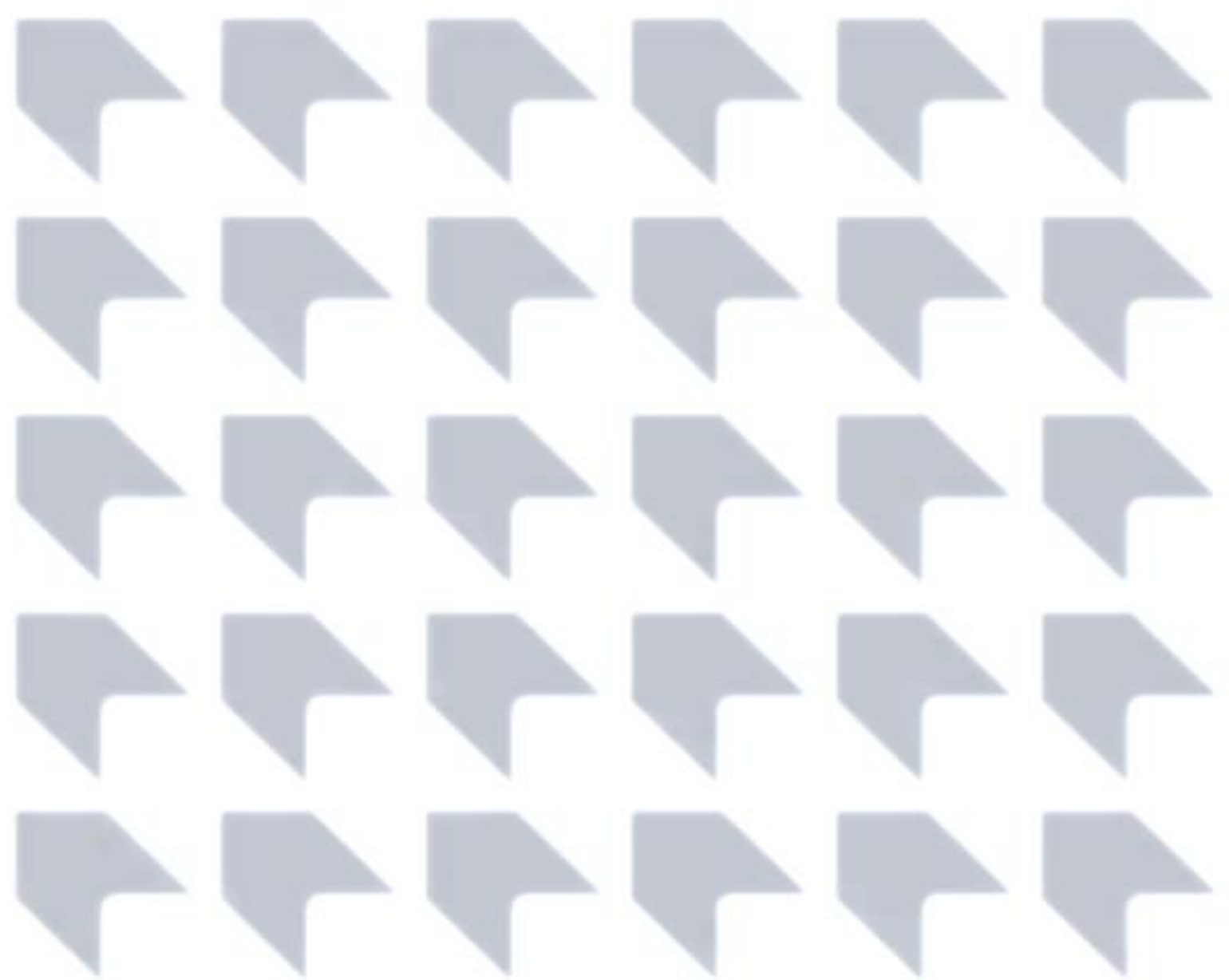




\section{Introduction}

As mentioned in the previous chapter, surface texturing and boundary slip can enhance the film thickness of lubricated sliding contacts and reduce friction.

In the first part of this chapter, the state of the art in texturing and a method to model and predict the effect of surface texturing on the film thickness will be discussed. In the second part, the effect of a limited lubricant film thickness on textured surfaces is shown. Finally, in the third part the effect of surface texturing when the slip boundary condition is present will be studied. In this chapter, it is shown that surface texturing can improve film formation. With the numerical model developed and by considering cavitation, the effect of shape, depth, size and textured area fraction on the frictional behaviour of parallel sliding lubricated contacts under conditions of mixed lubrication is studied. In addition, it is shown that by combining the surface texturing and boundary slip it is possible to enhance the film formation even more.

\subsection{Effect of surface texturing on film thickness}

Micro-geometric cavities have successfully been used to improve the lubrication between surfaces. By applying a texture at the surface, the lubricating fluid is pulled into the converging gap formed by the texture when the surfaces slide relative to each other, generating a pressure in the fluid. These micro-scale cavities can act as micro-reservoirs, can entrap wear particles, enhance the load carrying capacity and increase the film thickness, see for instance Etsion et al. [7, 9, 42]. Surface texturing also influences the transitions between the lubrication regimes [15]. As discussed in chapter 1 the use of laser texturing in the form of micro-grooves on, for instance, cylinder liners of internal combustion engines shows lower fuel consumption and wear [43-45]. The textured slider provides an effective larger film thickness than the non-textured surface, hence the slider is acting as a Rayleigh step bearing. Test results showed that the textured bearing operated with a film thickness that is about three times the film thickness of the nontextured bearing throughout the range of tested loads. In the work of Vilhena et al. [46], the performance of a reference non-textured surface and of textured discs with three different dimple depths are compared. The study is based on experiments. The results they obtained indicated that the beneficial effect of micro-dimples becomes greater with an increase in dimple depth. In the work of Yu et al. [11], a theoretical model based on a single dimple was established to investigate the geometric shape and orientation effects of circular, elliptical and triangular pockets on the hydrodynamic pressure generated between conformal contacting surfaces. Their results indicated that the geometric shape and orientation influences the load-carrying capacity of the contacting surfaces. With the same dimple area, area ratio and dimple depth the elliptical dimples with the major axis perpendicular to the sliding direction showed the best load-carrying capacity among all cases.

In a study by Kovalchenko et al. [15], the influence of texturing on the transitions between the different lubricating regimes was investigated. They observed that laser surface texturing (LST) is able to extend the range of the hydrodynamic lubrication regime in terms of load and sliding velocity. Removal of the bulges at the edges of the dimples by lapping after laser texturing is essential to optimize the positive effect of LST.

Based on the Reynolds equation, film thickness equations are developed for several configurations. From the generalized expression Eq. 1.1, the minimum film thickness in hydrodynamically lubricated contacts can be expressed a function of the applied normal load $F_{n}$ (contact pressure-p), sum velocity $u_{0}$, lubricant viscosity $\eta$ and macro geometry.

In this chapter a brief study of the effect of texturing (meso-geometry), including cavitation on the film formation for macroscopically plane-parallel sliding surfaces, is presented. For more information on this topic, an extended study is presented in paper $A$ of this thesis. 


\subsubsection{Mathematical modelling and solution:}

Four different texture patterns have been investigated in this study: circular pocket, triangular pocket, chevron and groove. Figure 2.1 shows the different cavity shapes, and the parameters characterizing their geometry. The chevron pattern is defined by two similar equilateral triangles of different size. The triangular pocket is a special case of the chevron with the inner edge length of the chevron equal to zero. For these two cases, the centre of the unit cell coincides with the midpoint of the altitude line of the triangle or chevron shape, see also [47, 48]. All patterns have a rectangular cross-sectional profile. The general film thickness formula can be written as (Eq. 2.1):

$$
h=h_{0}+h_{\text {macro }}+h_{\text {texture }}
$$

where $h_{0}, h_{\text {texture }}$ and $h_{\text {macro }}$ are shown in Fig. 2.1:

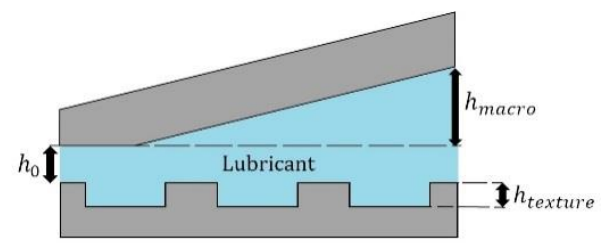

Figure 2.1. Geometrical scheme of macro and micro-scale film thickness terms.

In the case of flat-flat contact, the macro geometry is omitted and the film thickness (Eq. 2.1) reduces to (Eq. 2.2)[48]:

with:

$$
\frac{h(x, y)}{h_{0}(x, y)}=1+H(x, y)
$$

$$
H(x, y)=\frac{h_{\text {texture }}(x, y)}{h_{0}}
$$

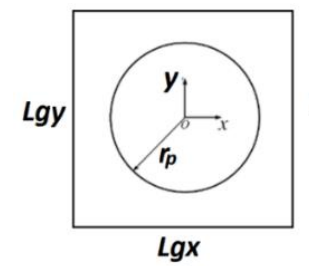

(a)

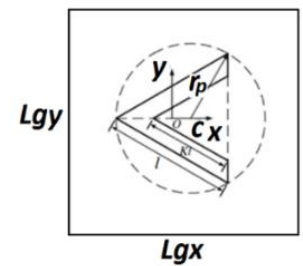

$\operatorname{Lg} x$

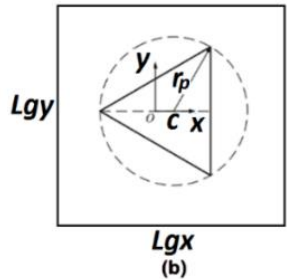

(b)

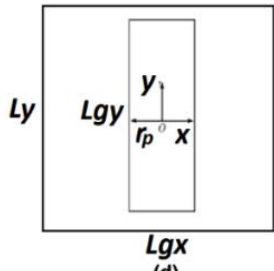

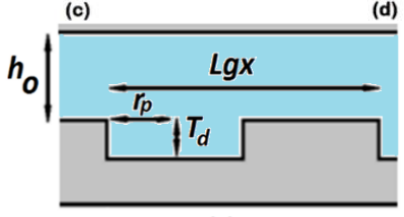

(e) 
Figure 2.2. Geometrical scheme of patterns, a) circular pocket, b) triangular pocket, c) chevron, d) groove and e) cavity profile [48].

$r_{p}$ is the characteristic radius for the triangular, circular pocket and chevron patterns and the half width of the grooves. The film thickness formula for the circular pocket is given in (Eq. 2.3):

$$
H(x, y)=\left\{\begin{array}{lc}
0, & \text { and } X^{2}+Y^{2}>1 \\
\frac{T_{d}}{h_{0}}, & X^{2}+Y^{2} \leq 1
\end{array}\right.
$$

The film thickness formula for triangular pocket can be written as (Eq. 2.4):

$$
H(x, y)=\left\{\begin{array} { c } 
{ 0 , \quad ( X , Y ) \notin \Omega } \\
{ \frac { T _ { d } } { h _ { 0 } } , \quad ( X , Y ) \in \Omega }
\end{array} , \Omega : - \frac { 3 } { 4 } \leq X \leq \frac { 3 } { 4 } \text { and } \left\{\begin{array}{c}
\frac{1}{\sqrt{3}} X-\frac{\sqrt{3}}{4} \leq Y \leq \frac{1}{\sqrt{3}} X+\frac{\sqrt{3}}{4} \\
-\frac{1}{\sqrt{3}} X-\frac{\sqrt{3}}{4} \leq Y \leq-\frac{1}{\sqrt{3}} X+\frac{\sqrt{3}}{4}
\end{array}\right.\right.
$$

The film thickness formula for the chevron can be written as (Eq. 2.5):

$$
H(x, y)=\left\{\begin{array} { c c } 
{ 0 , } & { ( X , Y ) \notin \Omega } \\
{ \frac { T _ { d } } { h _ { 0 } } , } & { ( X , Y ) \in \Omega }
\end{array} , \quad \Omega : - \frac { 3 } { 4 } \leq X \leq \frac { 3 } { 4 } \text { and } \left\{\begin{array}{c}
\frac{1}{\sqrt{3}} X+\frac{\sqrt{3}}{2}\left(K-\frac{1}{2}\right) \leq \\
Y \leq \frac{1}{\sqrt{3}} X+\frac{\sqrt{3}}{4} \\
-\frac{1}{\sqrt{3}} X-\frac{\sqrt{3}}{4} \leq \\
Y \leq-\frac{1}{\sqrt{3}} X+\frac{\sqrt{3}}{2}\left(\frac{1}{2}-K\right)
\end{array}\right.\right.
$$

where $K$ is the cavity width ratio and is equal to inner wall length over outer wall length. The film thickness formula for the grooves is given in (Eq. 2.6):

$$
H(x, y)=\left\{\begin{array}{cc}
0, & (X, Y) \notin \Omega \\
\frac{T_{d}}{h_{0}}, & (X, Y) \in \Omega
\end{array} \quad, \quad \Omega:-\frac{1}{2} \leq X \leq \frac{1}{2} \text { and } \frac{1}{2} \leq Y \leq \frac{1}{2}\right.
$$

In order to solve the Reynolds equation (Eq. 1.6), iterative solvers include nodal iteration, the tri-diagonal matrix algorithm (TMDA) is used, and the line-by-line TDMA solver (Patankar [49]) is applied so as to reduce the storage needed for calculation. When one solves a two-dimensional problem, the TDMA solution column-by-column or row-by-row becomes iterative, and sweeping is done line-by-line and column-by-column or row-by-row. For three-dimensional problems the TDMA is applied line-by-line on a selected plane and then the calculation is moved to the next plane, scanning the domain plane by plane [50].

\subsubsection{Results and discussion}

Several simulations are carried out to study the texturing patterns and to find the most efficient pattern and the effect of pattern orientation in the case of chevrons and triangular pockets. The film thickness for chevrons and triangles parallel to the moving direction and perpendicular to the moving direction is calculated. An extended study of the influence of surface texturing on film thickness is presented in paper A. The number of pockets and the distance between them are combined by introducing a new parameter, Pitch. The pitch for the chevron, triangle and circular pockets are calculated as: 
Texture size $=S=2 \times r_{p}$

Pitch in $\mathrm{x}$ direction: $P_{x}=\frac{S}{L_{g x}}$.

where the textured area $(10 \times 10 \mathrm{~mm})$ has the following geometrical data:

Table 2.1. Texturing properties.

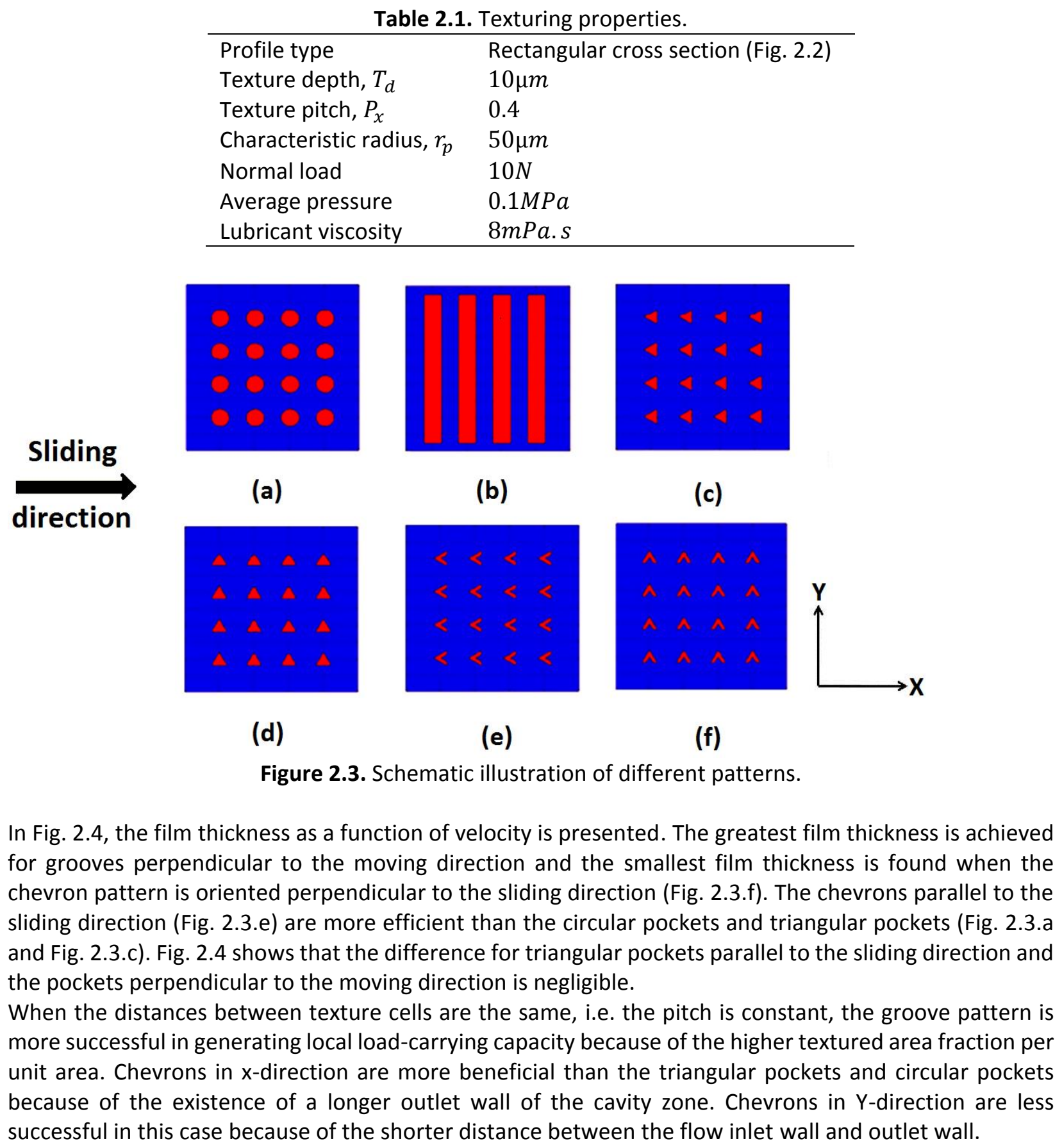




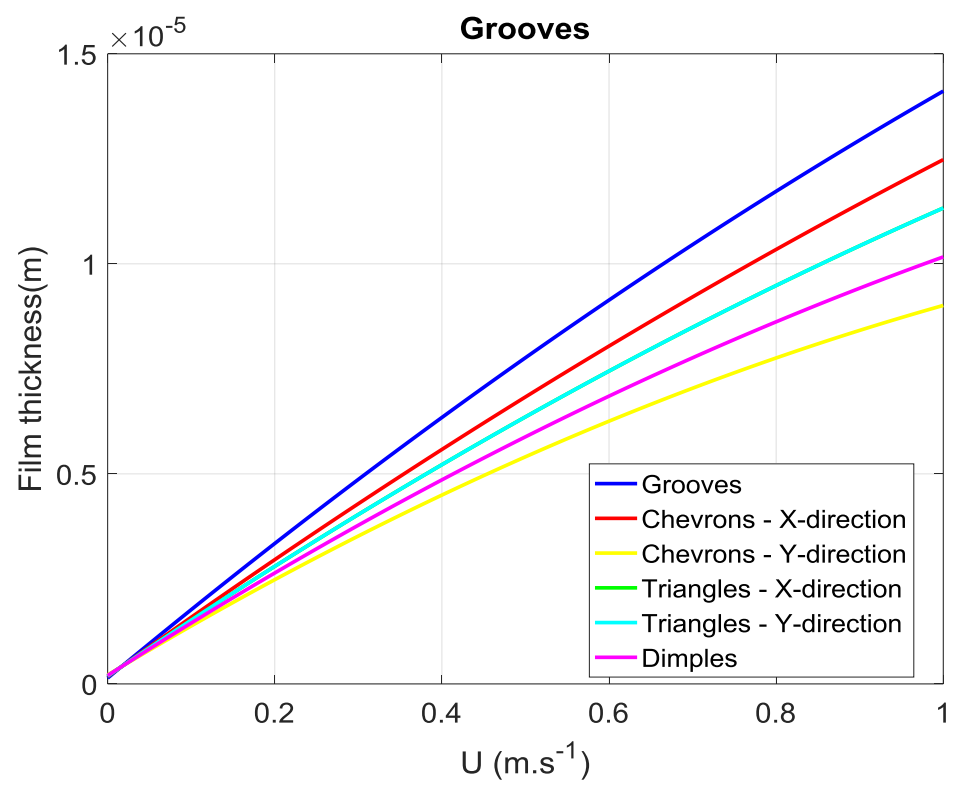

Figure 2.4. Comparison of film thickness as a function of velocity between different patterns, the triangle patterns are on top of each other.

\subsubsection{Optimum texture properties}

To gain the benefits of texturing surfaces an optimization of the geometrical parameters is performed. In this optimization, the effect of textured area fraction and cavity geometrical ratio on film thickness based on pockets with a rectangular profile (Fig. 2.2.e) is studied. The textured area operates under an average pressure $5 \mathrm{MPa}$ and the lubricant viscosity is $8 \mathrm{mPa}$. $\mathrm{s}$. In this section by using the following geometrical parameters, the effect of texturing on film thickness is studied:

$\xi: \quad$ Cavity geometrical ratio $=$ Cavity depth/Cavity length

$S_{p}: \quad$ Textured area fraction $=$ Cavity area/Cell area,

For circular pockets, these parameters can be written as (Eq. 2.7):

$$
\xi=\frac{T_{d}}{2 r_{p}}, \quad S_{p}=\frac{\pi r_{p}^{2}}{L_{g x} L_{g y}}
$$

For the triangular pockets (Eq. 2.8):

$$
\xi=\frac{T_{d}}{2 r_{p}}, \quad S_{p}=\frac{3 \sqrt{3} r_{p}^{2}}{4 L_{g x} L_{g y}}
$$

In Fig. 2.6, the simulation results show an optimum for the film thickness based on the texture area fraction $S_{p}$. When $S_{p}$ is tending to zero there is no film formation because of the absence of the wedge effect in a flat-flat contact and the lack of texturing, therefore there is no pressure generation. To analyze the effect of cavity geometrical ratio, simulations performed for the circular and the triangular pockets when $S_{p}$ is equal to 0.4 . Moreover, to study the effect of texture area fraction the cavity geometrical ratio is taken as 0.1 . 


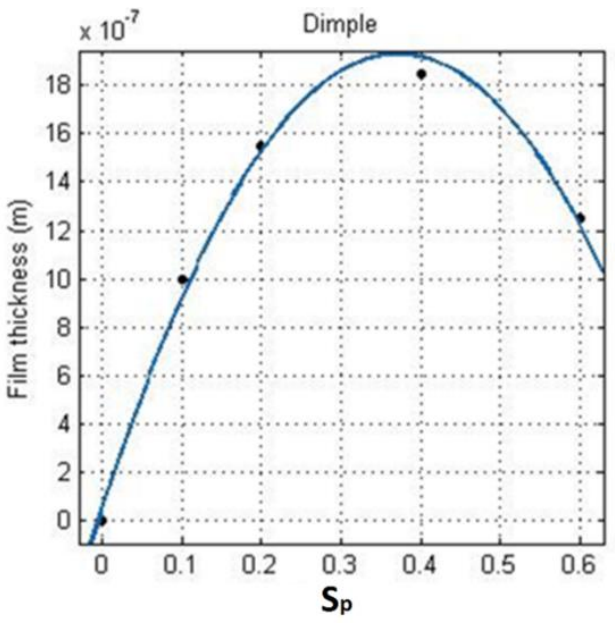

(a)

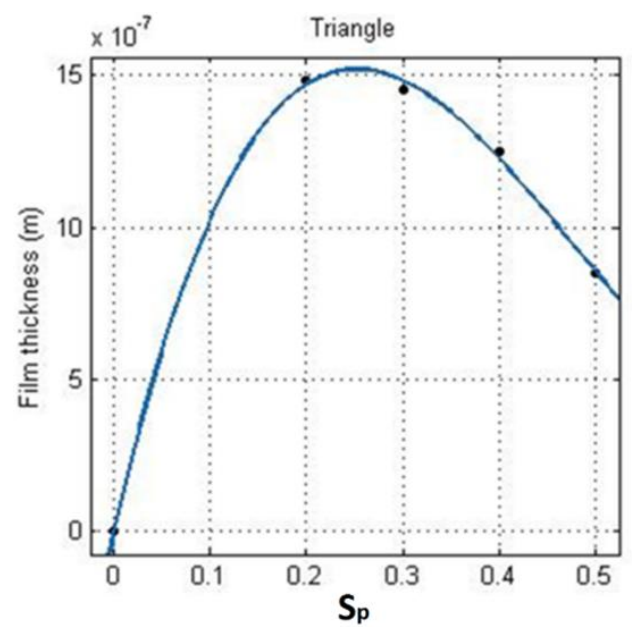

(b)

Figure 2.6. Film thickness curves as a function of textured area fraction $S_{p}$ for: a) circular pocket, b) triangular pocket.

The film thickness curves show, as a function of the variable cavity geometrical ratio ( $\xi$ ), an optimum (Fig. 2.7).

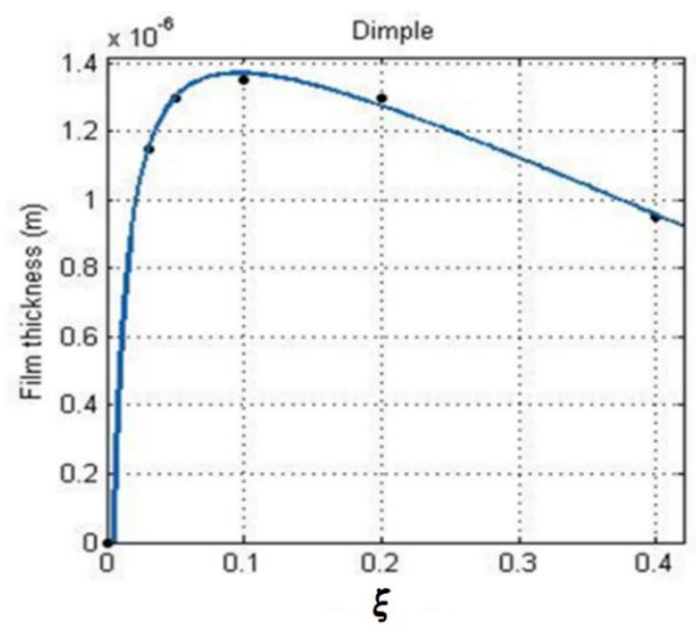

(a)

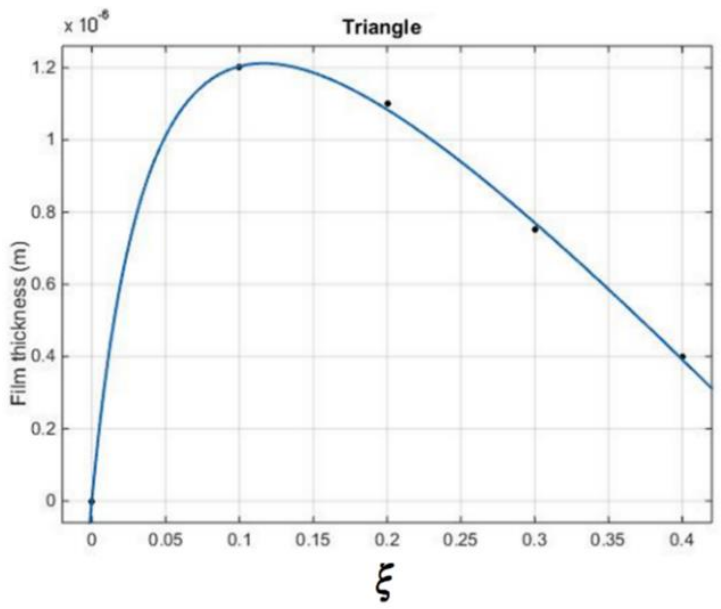

(b)

Figure 2.7. Film thickness as a function of cavity geometrical ratio $\xi$ for: a) circular pocket, b) triangular pocket.

In Fig. 2.7, a zero cavity ratio means zero depth, which resembles a flat-flat contact that results in no pressure generation and zero film thickness. After passing the optimum value of $\xi$ the higher ratio causes the pressure to drop because of the greater effect of cavitation and smaller texture size and as a result of a reduction in film thickness.

More information on the influence of surface texturing on film thickness can be found in paper A [48].

\subsubsection{Conclusions}

The aim of this section was to have a better understanding of film thickness for textured surfaces. Simulations of textured surfaces with different patterns and different texture properties are performed to calculate the lubricant film thickness in sliding contacts. The model is based on a numerical algorithm using 
the Reynolds equation with the Elrod cavitation algorithm formulation. The equations were discretized using the finite difference method and were solved using the TDMA iterative method. The effect of several parameters, such as pattern type, cavity cross-sectional shape, depth and size, on lubricant film thickness has been studied, along with the effect of lubricant leakage on the groove pattern. The groove pattern could generate a thicker lubricant film in contact than the other patterns studied in this work. The reason for this beneficial effect is the higher textured area fraction of the lubricated surface.

\subsection{Surface texturing on film thickness in starved lubricated contacts}

The study of starved lubrication, i.e. when a limited amount of lubricant is supplied to the contact, has attracted tribologists for many years [26-28, 30, 32, 33, 51].

It is observed that starvation can have a significant effect on the lubricant's film formation i.e. it can reduce the film thickness significantly. As a result, the lubricant cannot ensure a full separation of the two surfaces, leading to higher friction. As shown in the previous section, the tribological properties of the contacts are enhanced by applying a texture. Optimizing the texture dimensions makes it possible to retain the lubricant and improve the hydrodynamic effect [7, 9, 10, 15, 42, 51-55]. Starved lubrication has been investigated experimentally by several authors, such as Wedeven et al. [26], Pemberton et al. [56] and Kingsbury [27]. Further, Chiu [28] and Chevalier et al. [31], followed by Damiens et al. [32], have studied starved lubrication theoretically. These theoretical studies were based on the work of Jakobsson and Floberg [36] and Olsson [37], who introduced the concept of "fractional film content" and derived continuity relations. The influence of starvation on the lubrication of rigid cylinders was studied by Floberg [57]. Dalmaz and Godet [58] studied the influence of inlet starvation on the reduction in film thickness in the case of a sphere against a lubricated plate. In the work of Brewe and Hamrock [59], the influence of starvation was studied theoretically. In this work, by using the Reynolds boundary condition and by systematically reducing the fluid inlet level, they observed a pressure build-up for a given film thickness. In their study, the inlet meniscus boundary was taken as the start of the pressure build-up. They analyzed a wide range of geometry parameters from a ball on a plate to a ball in a conforming groove. The film thickness formula was modified to incorporate the starvation effect. Boness [60] showed experimentally the importance of the starvation effects on the bearing failure and wear.

\subsubsection{Model}

The mathematical method and governing equations that are used to model starved lubrication in sliding textured surfaces are the same as used in the previous section; this is explained comprehensively in paper $\mathrm{B}$ of this thesis [51]. To investigate the effect of limited lubricant supply $\left(h_{\text {oil }}\right)$ on the film thickness for different texturing parameters and for different patterns several simulations were performed.

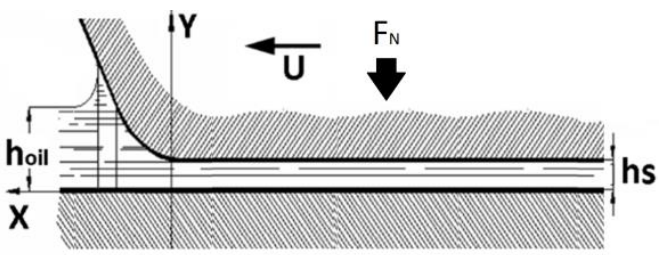

Figure 2.8. Schematic illustration of limited lubricant supply $\left(h_{\text {oil }}\right)$ and calculated film thickness $\left(h_{s}\right)$ [51].

The effect of texturing parameters i.e., pattern type, texture pitch $\left(P_{x}\right)$, texture depth $\left(T_{d}\right)$, and texture size $(S)$, on the starved film thickness $\left(h_{s}\right)$ is given in sub-section 2.3.2. These simulations were based on surfaces with grooved pockets.

The results for different values of the lubricant supply thickness $\left(h_{\text {oil }}\right)$ for different texturing patterns is given in sub-section 2.2.3. 


\subsubsection{Texturing parameters}

The starved film thickness for different values of $T_{d}$ and $S$ is presented in Fig. 2.9, these calculations are based on $h_{\text {oil }}=2.5 \mu m$, (relevant other data see table 2.1).

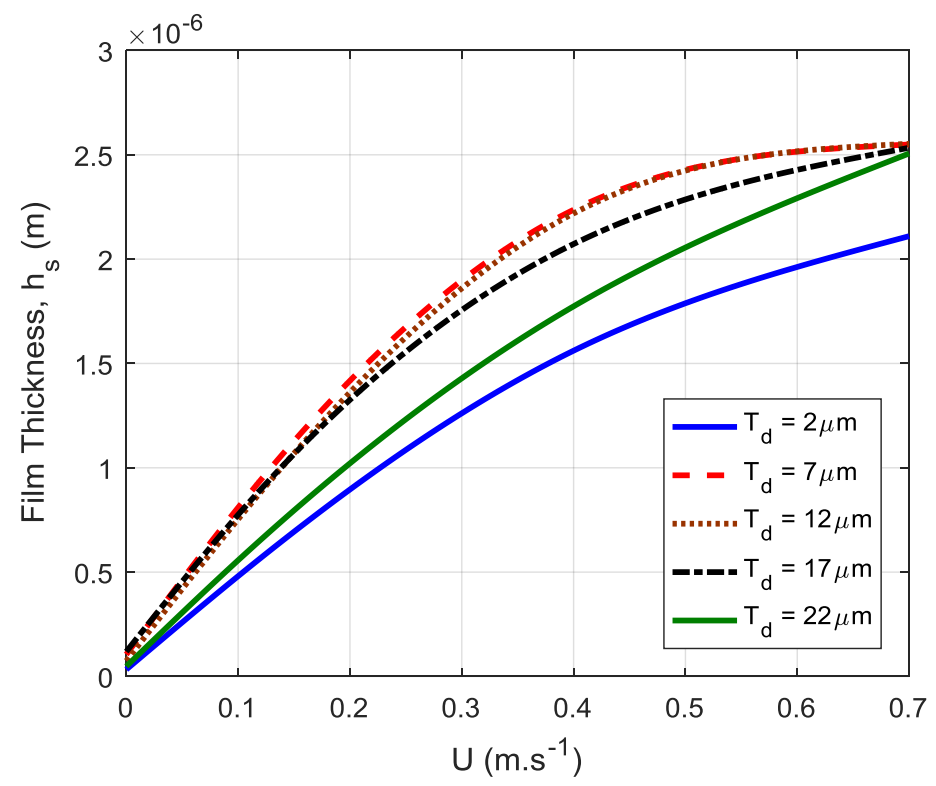

(a)

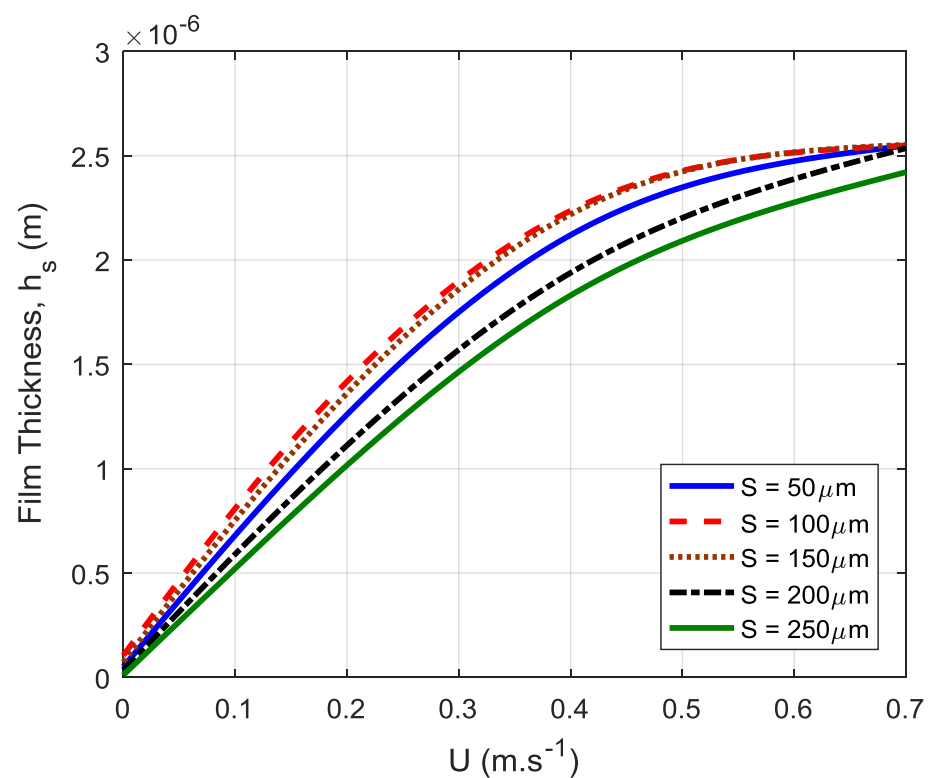

(b)

Figure 2.9. Film thickness as a function of velocity obtained for grooves with $h_{\text {oil }}=2.5 \mu \mathrm{m}$, for different values of a) grooves depth $T_{d}$ with $S=100 \mu \mathrm{m}$ and b) grooves size $S$ with $T_{d}=7 \mu \mathrm{m}$.

Figure 2.9.a shows the effect of a limited lubricant supply on starved film thickness for different values of the texture depth parameter $\left(T_{d}\right)$. When the sliding is in the lower velocity range $\left[0 \mathrm{~m} . \mathrm{s}^{-1}-0.3 \mathrm{~m} . \mathrm{s}^{-1}\right]$, the film thickness was more sensitive to increasing texture depth than when the sliding velocity was in the higher velocity range $\left[0.3 \mathrm{~m} . \mathrm{s}^{-1}-0.7 \mathrm{~m} . \mathrm{s}^{-1}\right]$. The influence of texture size $(S)$ on the starved film thickness 
is shown in Figure 2.9.b. From this calculation for starved lubricated contacts, it is shown that by increasing the velocity the influence of optimum cavity size on film formation fades and cavities with different sizes can have the same film thickness in higher velocities.

\subsubsection{Comparison surface patterns}

In Figure 2.10, the film thickness as a function of velocity is shown for different patterns with $T_{d}=7 \mu \mathrm{m}$, $S=100 \mu m$, and $P_{x}=0.4$ for different values of the lubricant supply thickness $\left(h_{\text {oil }}\right)$, i.e. $2,2.5,3$ and $3.5 \mu \mathrm{m}$.

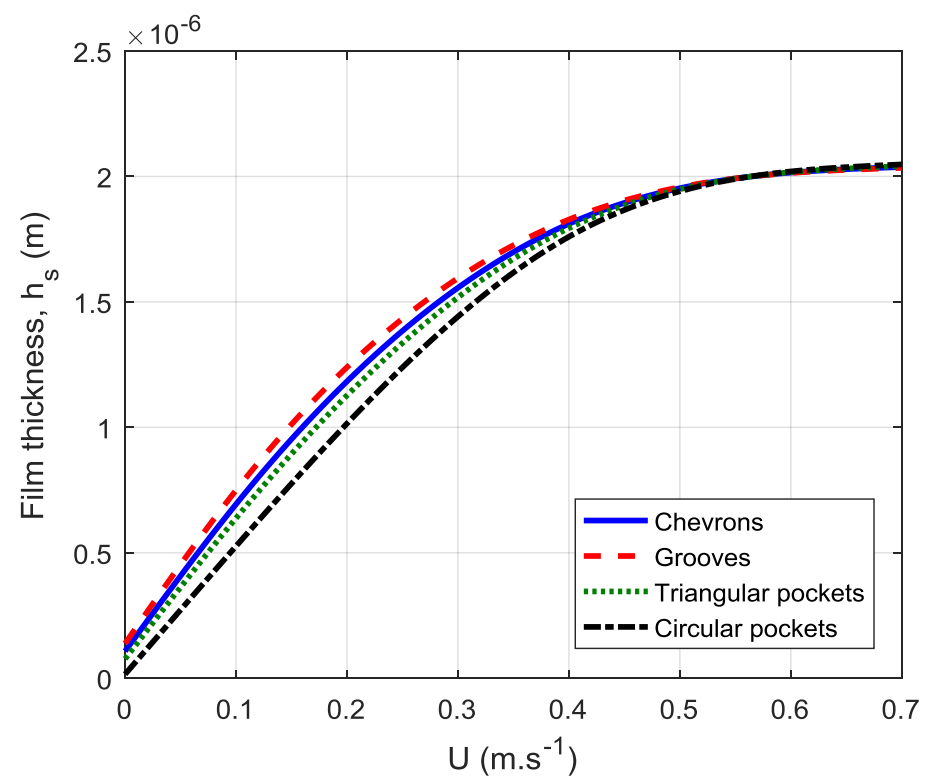

(a)

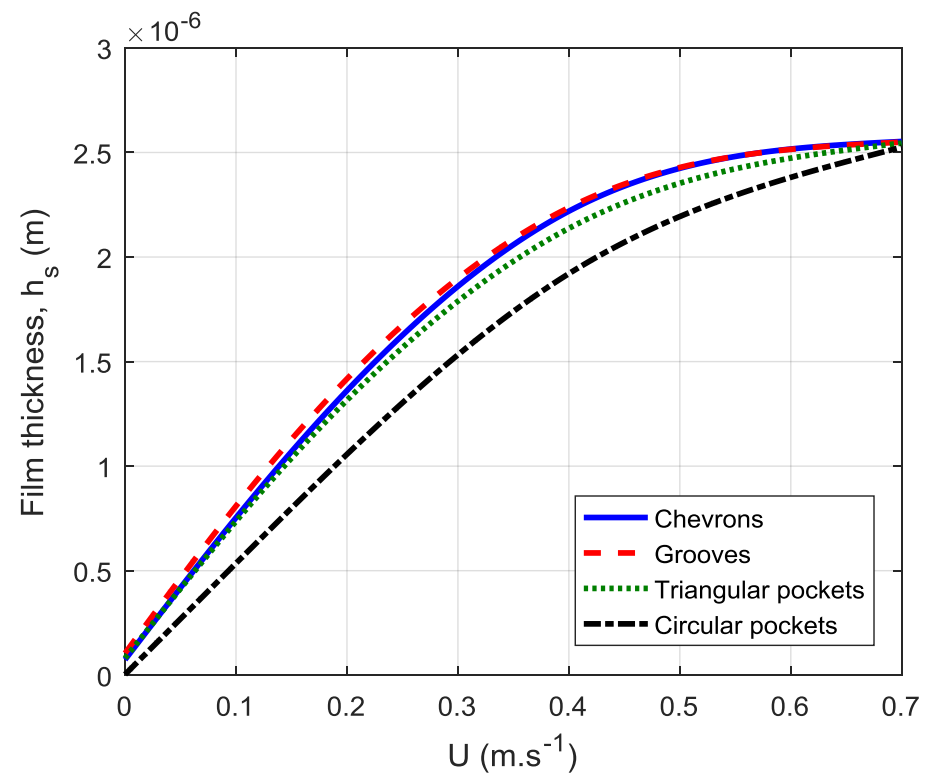

(b) 


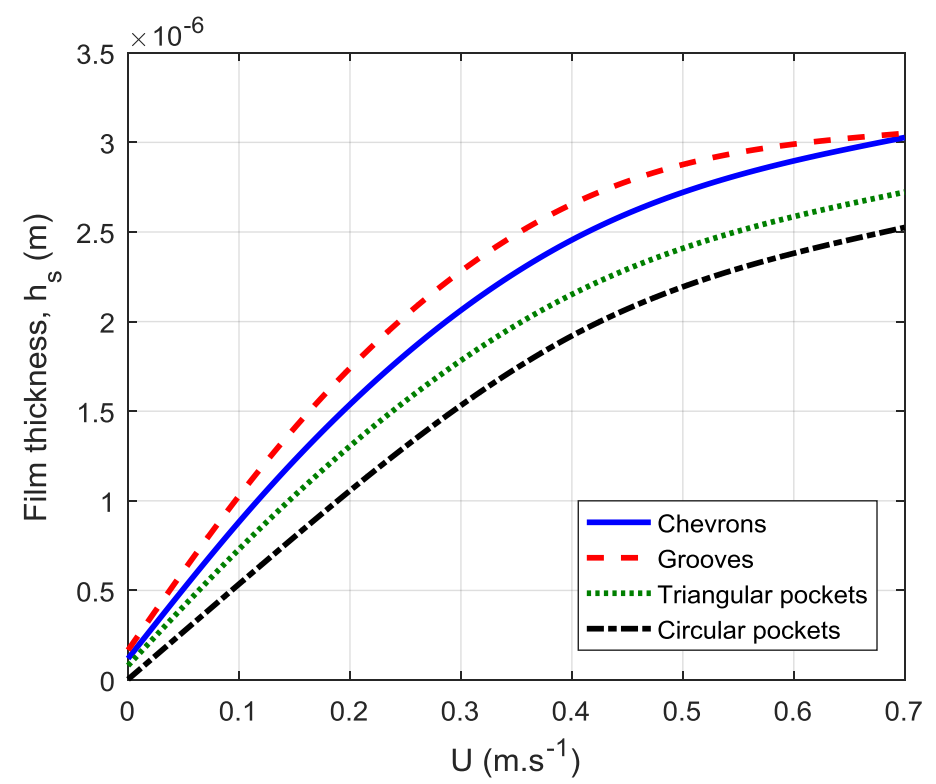

(c)

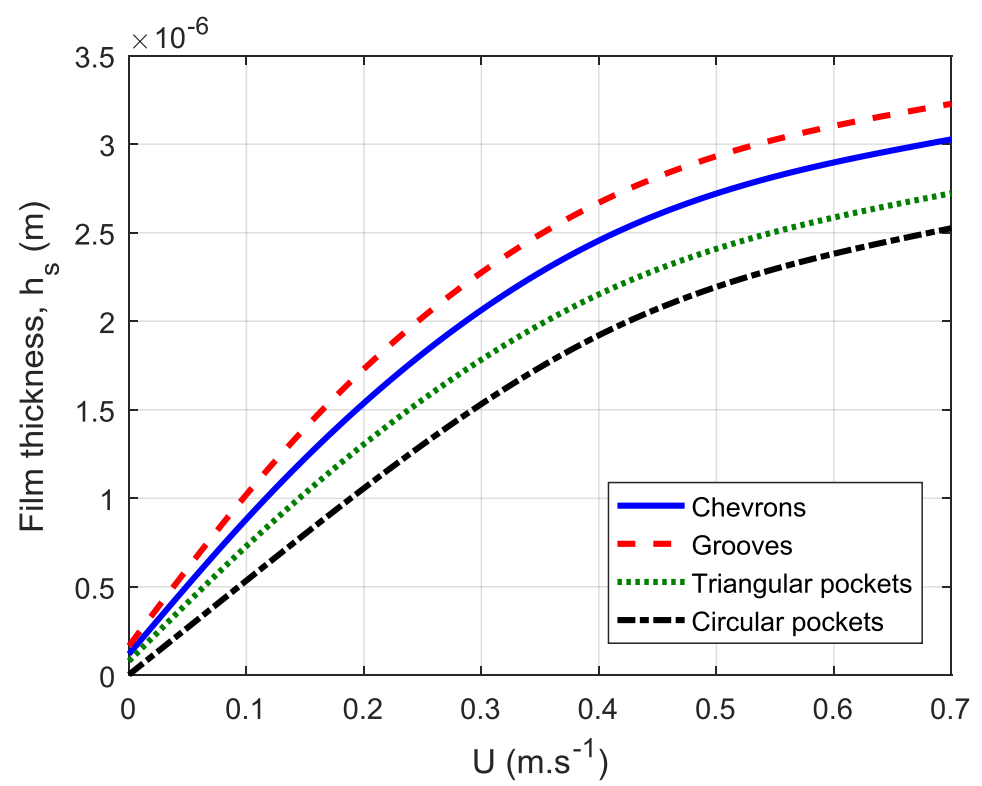

(d)

Figure 2.10. Effect of limited lubricant supply on the film thickness as a function of velocity for different texturing patterns, for: a) $\left.h_{o i l}=2 \mu \mathrm{m}, \mathrm{b}\right), h_{o i l}=2.5 \mu \mathrm{m}$ c) $h_{o i l}=3 \mu \mathrm{m}$ and d) $h_{o i l}=3.5 \mu \mathrm{m}$.

For different levels of lubricant supply, the groove pattern generates the greatest film thickness. The chevron pattern produced a thicker film than the triangular and circular pockets. The lowest film thickness was obtained by employing the circular pocket pattern. In Figure 2.10.a, when $h_{\text {oil }}$ was equal to $2 \mu m$, the effect of starvation on the calculated film thickness for the different patterns is the same.

By increasing $h_{\text {oil }}$, the effect of starvation on the film thickness decreases. For instance, when $h_{\text {oil }}=$ $3.5 \mu \mathrm{m}$ (see Figure 2.10.d), the difference in film thickness found for the different texturing patterns is clear at high and low velocities, unlike for $h_{\text {oil }}=2.0 \mu \mathrm{m}$ (Figure 2.10.a) where at higher velocities there was no difference between the film thickness generated by the different patterns. In this case, the groove pattern 
has the most promising effect for gaining a greater film thickness. When the distance between the texture cells is the same, i.e. the pitch is constant, the groove pattern is more successful in generating load-carrying capacity because of the higher textured area fraction per unit area. Chevrons are more efficient than triangular pockets and circular pockets because of the existence of a longer outlet wall in the cavity zone. In summary, the results in Figure 2.10 show that the greatest film thickness was achieved for the grooves and the smallest film thickness was found for circular pockets. The chevrons are more efficient than the triangular pockets.

\subsubsection{Conclusions}

In this section, the effect of starvation on film formation of textured contacts as well as the influence of texturing parameters like pattern type, cavity depth and cavity size on film thickness of starved lubricated contacts have been studied and the following conclusions drawn:

- For different lubricant supply values $h_{\text {oil }}$, the groove pattern shows the greatest film thickness at low velocities. The chevron pattern generates a greater film thickness than the triangular and circular pockets and the smallest film thickness has been found for the circular pocket pattern.

- For small values of the applied lubricant thickness, the effect of starvation on the film thickness is greater. Increasing the lubricant supply $\left(h_{\text {oil }}\right)$ gives the texture geometry and dimensions greater influence on the film thickness.

More information about the influence of surface texturing on the film thickness in starved lubricated parallel sliding contacts is provided in paper B of this thesis.

\subsection{Boundary slip on film thickness}

In the previous sections, the effect of surface texturing on film thickness and starved lubrication has been studied. In this section, the influence of applying boundary slip at the surface in a lubricated sliding contact will be investigated. Boundary slippage can be presented as an alternative for friction reduction.

The no-slip boundary condition assumption that the first layer of fluid molecules has the same velocity as its contacting solid surface has been widely accepted in the field of fluid mechanics, although the classical fluid-dynamic assumption of a no-slip boundary condition (i.e., relative zero flow velocity at a solid wall) is quite satisfactory in dealing with most viscous flow problems of continuum fluids, molecular dynamics simulations have shown that microscopic slip is possible, depending on several fluid-solid interfacial parameters [61-63]. Slip of a liquid at a solid wall is especially important for many engineering topics involving liquid-solid interfacial phenomena, such as lubrication, flow through porous media and liquid coatings. Many surfaces in nature are highly hydrophobic. The best-known example of a hydrophobic selfcleaning surface are the leaves of the lotus plant [64]. On such surfaces, the contact of the liquid with the solid roughness is minimal, while most of the interface is a liquid-gas one, resulting in existence of a relative velocity between the surface and lubricant and reduced friction [65-67].

In the work of Tauviqirrahman et al. [17], the effect of boundary slip on friction reduction is investigated. In that study a combined optimized complex slip surface and optimized slope incline ratio is found. It was shown that surface optimization of a lubricated parallel sliding contact with a slip surface can double the hydrodynamic load-carrying capacity and reduce the friction force by half of what the classical Reynolds theory predicts for an optimal inclined slope traditional slider. For a two-dimensional (finite length) journal bearing $\mathrm{Ma}$ et al. [18] showed that the optimization of shape and size of the surface may enhance the frictional behaviour. In this section the friction is discussed using the two-component slip model for textured surfaces. 


\subsubsection{Mathematical solution of the model}

In simulating the boundary slip phenomenon the main challenge is to choose the most realistic slip model and to apply the most accurate boundary conditions for a hydrophobic surface.

A boundary condition for partial slip was proposed by Navier in 1823. This boundary condition is the most frequently used model to characterize boundary slip. Navier introduced a linear boundary condition, which states that the liquid velocity term $u_{z=0, h}$ at the wall surfaces is proportional to the shear stress at the surface. The slip length, $b$, is defined as the distance beyond the liquid/solid interface as shown in Fig. 2.11.

$$
u_{z=h}=u_{s}=b \frac{\tau_{x}}{\eta}=\left.b \frac{d u}{d z}\right|_{z=h}
$$

where $b$ is the slip length, $u_{s}$ is the slip velocity of the fluid along the surface $x$-axis, $u$ is Cartesian velocity component in $x$-direction, $\eta$ is bulk viscosity and $\tau_{x}$ is shear stress in x-direction.

Experimental studies with wall slip make use of surfaces with different physical or chemical treatments together with different types of lubricants in order to achieve different solid/liquid interfacial properties. Another common method of changing the property of the solid/liquid interface is by adding surfactants to the liquid lubricant. The velocity of the fluid flow at the wall surface can be adjusted effectively by using different concentrations of surfactants [68].

Spikes and Granick [69] introduced a mathematical slip model for Newtonian fluids. Their model $\tau_{c}=$ $\tau_{c o}+\frac{\eta}{b} u_{s}$ incorporates both the critical shear stress and constant slip length criteria in which $\tau_{c o}$ is the critical shear stress at the onset of slip. The critical shear stress model adopted in the present study assumes that wall slip occurs only after the surface shear stress reaches the critical shear stress. The focus of this section is to show the importance of the critical shear stress choice for contacts with a textured surface combined with a slip boundary.

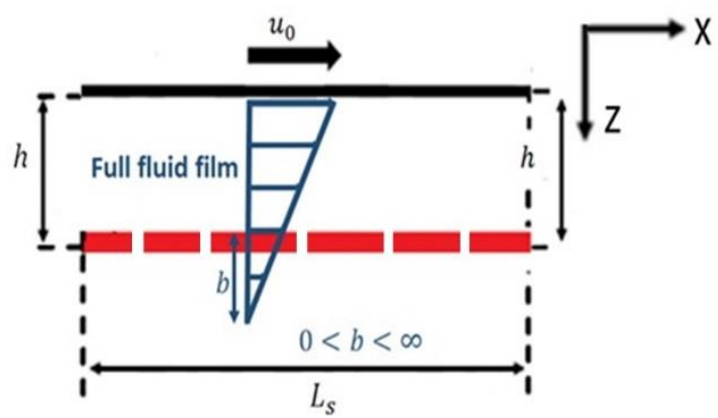

Figure 2.11. Schematic of wall slip model, boundary slip condition at $z=h$ (red dashed line) [70].

The governing equation for boundary slip condition is derived from the force equilibrium analysis of a fluid element by applying the flow continuity, as it is presented in Eq. 2.10 [70]:

$$
\frac{\partial}{\partial x}\left(h^{3} \frac{\partial p}{\partial x}\right)+\frac{\partial}{\partial y}\left(h^{3} \frac{\partial p}{\partial y}\right)=6 \eta\left(u_{0} \frac{\partial h}{\partial x}+\frac{\partial\left(h u_{s}\right)}{\partial x}+\frac{\partial\left(v_{s} h\right)}{\partial y}\right)
$$

$u_{s}$ and $v_{s}$ are the slip velocity of the surface in $x$ and $y$ direction respectively. By defining the following dimensionless parameters:

$$
X_{s}=\frac{x}{L}, Y_{S}=\frac{y}{B}, H=\frac{h}{h_{0}}, U_{s}=\frac{u_{s}}{u_{0}}, V_{s}=\frac{v_{s}}{u_{0}}, P=\frac{p-p_{C}}{p_{0}}, W=\frac{6 \eta u_{0} L^{2}}{p_{0} h_{0}^{2}}
$$


Eq. 2.10 becomes:

$$
\left(H^{3} \frac{\partial P}{\partial X_{S}}\right)+\frac{L^{2}}{B^{2}} \frac{\partial}{\partial Y_{S}}\left(H^{3} \frac{\partial P}{\partial Y_{S}}\right)=W\left(\frac{1}{L} \frac{\partial H}{\partial X_{S}}+\frac{1}{L} \frac{\partial\left(H U_{S}\right)}{\partial X_{S}}+\frac{1}{B} \frac{\partial\left(H V_{S}\right)}{\partial Y_{S}}\right)
$$

For more information about how to derive this equation the reader is referred to paper $\mathrm{E}$ of this thesis.

\subsubsection{Boundary conditions}

As mentioned before, when applying boundary slip on the stationary surface it is possible to improve the tribological behaviour $[17,19,20,70,71]$, i.e. to achieve a higher load carrying capacity for the parallel sliding surface situation (see Fig. 2.12).

In this study, to achieve a higher film thickness the optimum slip area dimensions are taken from work of Tauviqirrahman et al. [72] The starting point of the slip area is intended to be at the starting point of the textured area and the reason for this alignment is to maximize the effect of partial slip [19].

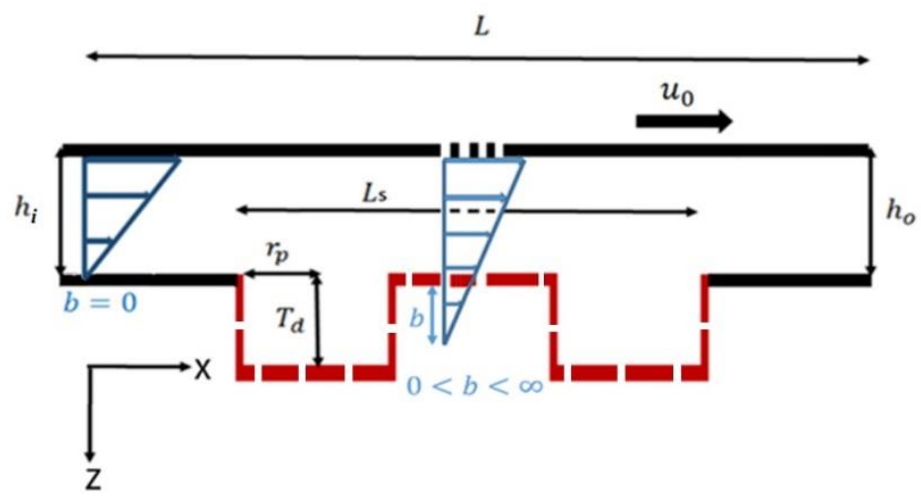

Figure 2.12. Parallel sliding contact (schematic); the red dashed line indicates part of the surface with boundary slip [70].

In the slip area, due to the existence of slip velocity at the lower surface the boundary conditions are:

$$
\left\{\begin{array}{l}
z=h \rightarrow u=u_{s}, v=v_{s} \\
z=0 \rightarrow u=u_{0}, v=0 \\
h_{i}=h_{o}=h \quad \text { (parallel sliding surfaces) }
\end{array}\right.
$$

In the no-slip region, boundary conditions are:

$$
\left\{\begin{array}{l}
z=h \rightarrow u=0, v=0 \\
z=0 \rightarrow u=u_{0}, v=0 \\
h_{i}=h_{o}=h
\end{array}\right.
$$

where $h_{i}$ and $h_{o}$ are the film thickness at the inlet and outlet of the contact respectively; these are equal for parallel sliding contacts.

\subsubsection{Slip velocity equation}

The two-component slip model reads [70]: 


$$
\tau_{c}=\tau_{c o}+\frac{\eta}{b} u_{s}
$$

By applying the boundary conditions (Eq. 2.12) for the slip area and based on the two component model of Spikes and Granick [73] (Eq. 2.14) it is possible to obtain the slip velocity $\left(u_{s}\right)$ :

$$
u_{s}=\frac{\left(u_{0}-\frac{h^{2}}{2 \eta} \frac{\partial p}{\partial x}+\operatorname{sgn}\left(u_{s}\right) \frac{h}{\eta} \tau_{c o}\right)}{1+\frac{h}{b}}
$$

\subsubsection{Friction calculation}

The friction force $\left(F_{f}\right)$ generated in a lubricated system due to shearing is calculated by integrating the shear stress over the interface surface area. To calculate the coefficient of friction, we have:

$$
\mu=\frac{F_{f}}{F_{T}}
$$

To understand the boundary slip effect on frictional behaviour of textured surfaces a normalized coefficient of friction is introduced $\left(\mu^{*}\right)[70]$ :

$$
\mu^{*}=\frac{\mu}{\mu_{\text {ref }}}
$$

$\mu$ is the calculated coefficient of friction when slip in the contact exists $(b>0)$ and $\mu_{r e f}$ is the coefficient of friction when there is no slip area in the entire contact $(b=0)$.

\subsubsection{Results and discussion}

In this sub-section, the influence of the boundary slip parameters slip length $(b)$ and critical shear stress $\left(\tau_{c o}\right)$ on the film thickness for textured surfaces is studied. The slip area length $L_{s}$ in these calculations is taken as $75 \%$ of contact length based on the study of Tauviqirrahman et al. [72] on the optimization of the boundary slip area. The calculation example given here deals with the groove pattern as shown in Fig. 2.13 and operation parameters given in table 2.2.

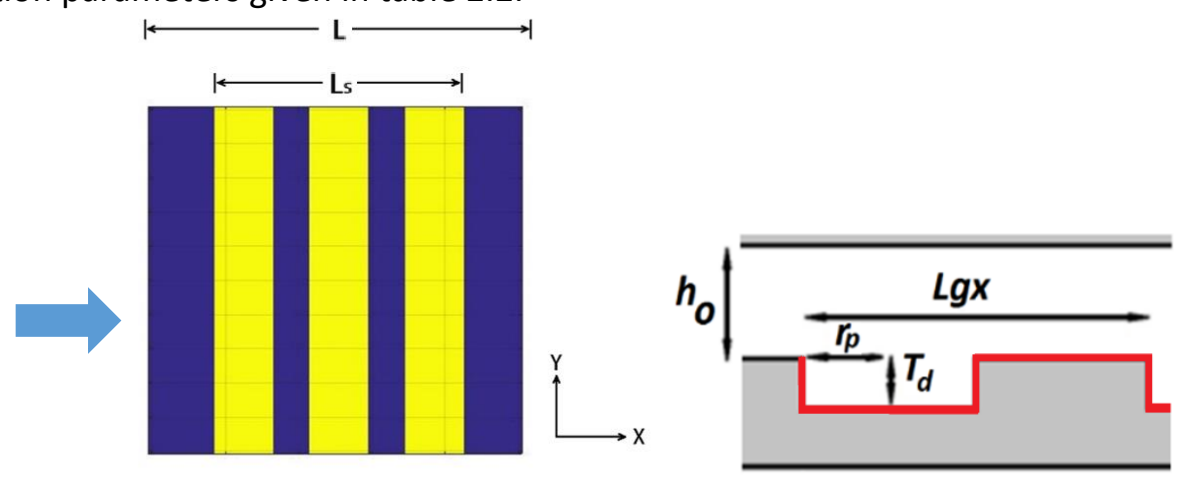

Figure 2.13. Texture pattern (open groove pattern) [70].

Table 2.2. Range of operating parameters.

Parameter Value Unit




\begin{tabular}{llc}
\hline Texture depth $\left(T_{d}\right)$ & 10 & $\mu m$ \\
Texture size $(S)$ & 150 & $\mu m$ \\
Texture pitch $\left(P_{x}\right)$ & 0.5 & - \\
Viscosity & 8 & $m P a . s$ \\
Load & 20 & $N$ \\
Slip length $(b)$ range & $0-30$ & $\mu m$ \\
Slip area length $\left(L_{s} / L\right)$ & 0.75 & - \\
Contact area & $1 \times 10^{-6}$ & $\mathrm{~m}^{2}$ \\
\hline
\end{tabular}

In table 2.2 the range of slip length and critical shear stress is based on work of Tauviqirrahman et al. [17] on parametric optimization of complex slip surfaces.

\subsubsection{Effect of Slip length $(\boldsymbol{b})$}

In order to study the influence of the slip length the texturing parameters $\left(T_{d}, S\right.$ and $\left.P_{x}\right)$ are set as constant and the slip length $(b)$ is varied from 0 to $30 \mu m$.

As can be seen in Fig. 2.14, the increase of the slip length leads to a significant improvement in the film thickness. However, after the slip length passes $20 \mu \mathrm{m}$, the increase in this parameter causes a smaller improvement in film thickness in comparison with values of slip length of less than $20 \mu \mathrm{m}$.

The existence of a greater slip length can cause a higher slip velocity on a slippery wall, which results in a larger velocity difference between the fluid velocity on areas with boundary slip and on non-slip areas. This difference in velocity will cause a pressure gradient, which results in a greater film thickness.

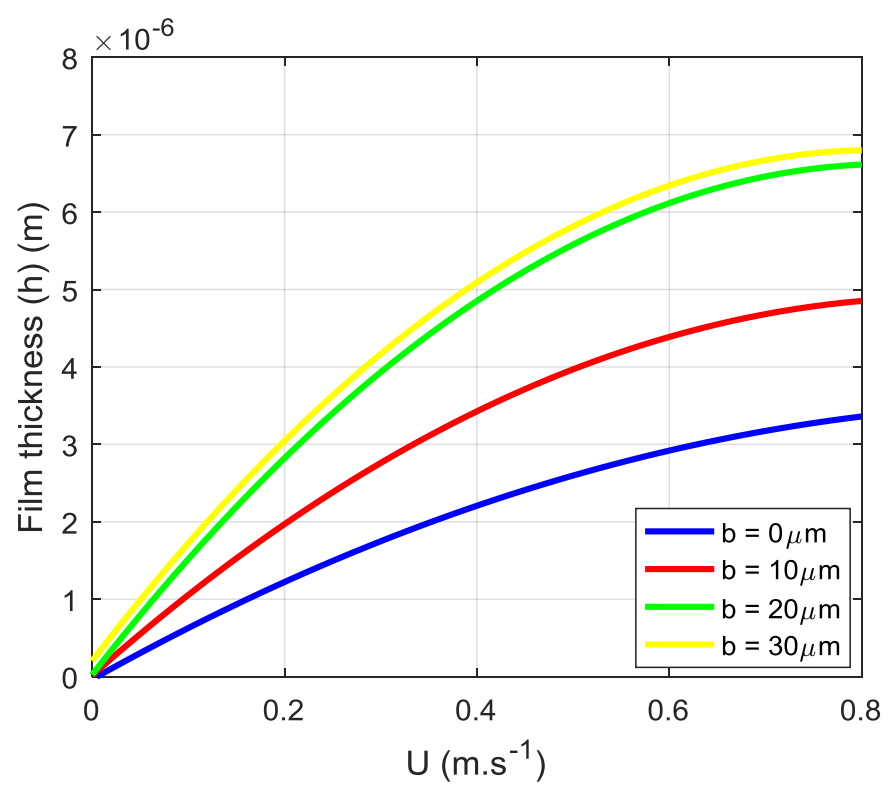

Figure 2.14. Influence of slip length $(b)$ on film thickness. 


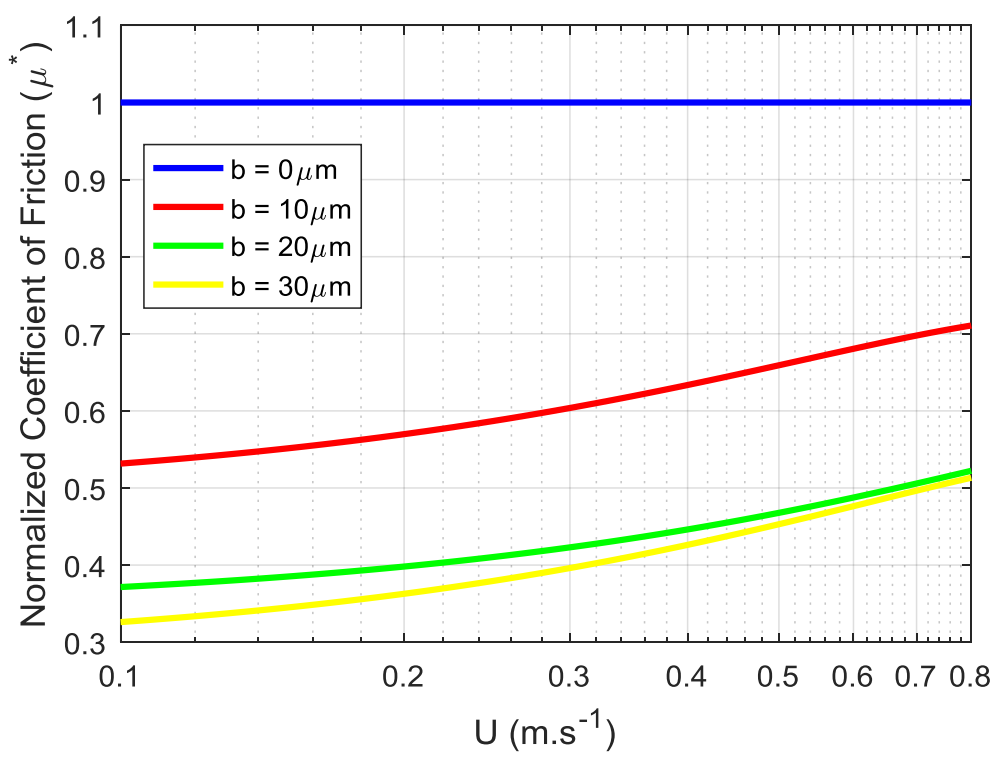

Figure 2.15. Influence of slip length $(b)$ on friction.

In Fig. 2.15, the effect of the variation of the slip length on the frictional behaviour of textured surfaces is presented. The coefficient of friction as a function of velocity is calculated for four different values of the slip length. The normalized coefficient of friction is calculated by dividing the calculated values by the coefficient of friction for the no-slip condition $\left(\mu_{r e f}=\mu_{\mathrm{b}=0}\right)$. This normalization is used to indicate the frictional behaviour of contacts at different slip lengths.

With respect to the normalized coefficient of friction $\left(\mu^{*}\right)$, the increase in the slip length leads to a decrease in this parameter $\left(\mu^{*}\right)$. It can also be observed that if the slip length decreases to zero, $\mu^{*}$ leads to its noslip value. Fig 2.15 shows that the rate of friction drop is similar to that found for the film thickness.

The reader is referred to paper $E$ for the effect of critical shear stress on the film thickness and friction.

\subsubsection{Conclusion}

From the study of the influence of slip length on the film thickness and friction, it is clear that a thicker lubricant film is achievable due to slip. With respect to the normalized coefficient of friction $\left(\mu^{*}\right)$, the increase in the slip length leads to a decrease in friction.

\subsection{Summary}

The numerical code developed so as to have a better understanding of film thickness for textured surfaces is used to study the effect of starvation on the film thickness for textured surfaces. A study is also made of the influence of boundary slip and its related properties, such as slip length and critical shear stress, by modifying the Reynolds equation and numerical code on film formation and friction. The effect of several parameters on lubricant film thickness, such as pattern type, depth and size, has been studied. The groove pattern shows the highest lubricant film formation. The reason for this beneficial effect is the higher textured area.

Furthermore, the effect of texturing parameters on the starved film thickness has been studied. For different lubricant supply values $h_{\text {oil }}$, the groove pattern shows the greatest film thickness.

From the study on influence of slip length on film thickness and friction, it is shown that a thicker lubricant film, and therefore a lower friction, is achievable when the slip length has higher values, due to the higher slip velocity. 


\section{Chapter 3}

The Effect of Surface texturing on frictional behaviour of Sliding Contacts

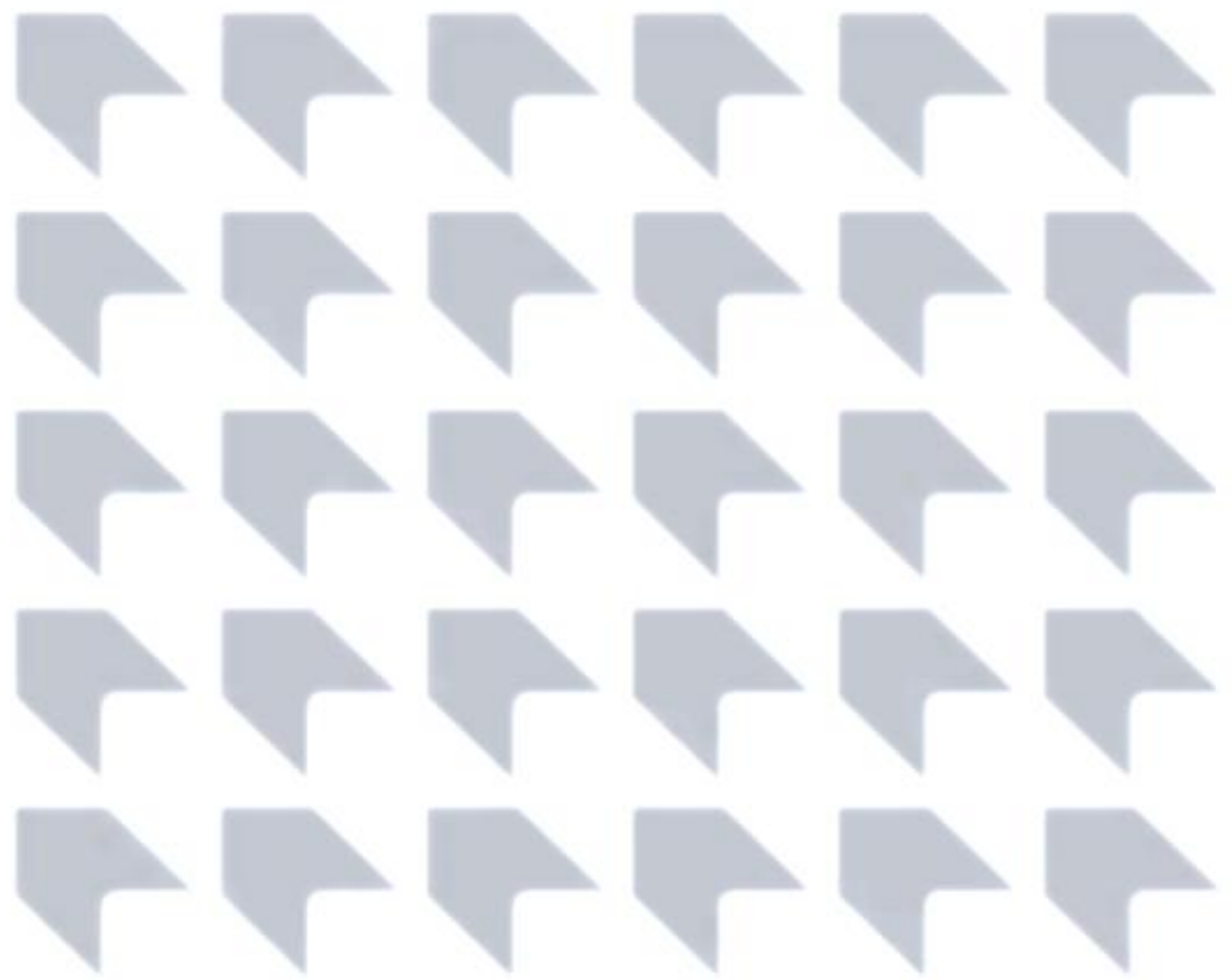




\section{Introduction}

In many industrial applications, a modification of the surface geometry can enhance the tribological behaviour of lubricated sliding contacts to a favourable condition. Therefore, there is a growing demand for improving the tribological performance (frictional behaviour) of sliding lubricated contacts. The first method of reducing the friction is by making the surfaces more smooth; however, producing a super smooth surface can be expensive and can cause failure [74]. In the case of lubricated contacts, a geometrical modification on micro-meso scale of the surfaces can be a potential solution. Surface texturing has been widely applied as a well-known method to reduce friction and wear between sliding surfaces separated by a thin lubricant film.

In this chapter, the effect of surface texturing on the coefficient of friction in parallel sliding lubricated surfaces is investigated. It is shown that surface texturing can improve film formation, and as a result the load-carrying capacity, and can also reduce the coefficient of friction. With the numerical model developed and by considering cavitation, the effect of shape, depth, size and textured area fraction on the frictional behaviour of parallel sliding lubricated contacts under conditions of mixed lubrication is studied. Furthermore, the effect of surface texturing and related parameters on the coefficient of friction for starved lubricated parallel sliding contacts is studied and the results are presented.

\subsection{Surface texturing and frictional behaviour}

In recent decades, much effort has been put into understanding the effect of surface texturing on improving the tribological performance of lubricated contacts $[1,6,16,45,54,75-79]$. The cavities can result in an improvement in tribological performance due to local convergent and divergent gaps, which can generate pressure even in lubricated parallel sliding flat-on-flat contacts. In the cavities, cavitation may occur. Therefore, three important effects can occur to enhance the tribological performance of lubricated sliding textured surfaces. The first effect is the increased hydrodynamic pressure in the contacts due to the converging wedges constituted by the cavities or pockets. The second is the containment of wear debris inside the cavities, which can be beneficial by removing the wear particles between the two surfaces so as to avoid abrasive wear. The third effect is the existence of pockets filled with a lubricant which could be particularly useful in conditions involving high plastic deformation [5].

A lot of studies related to surface texturing with respect to friction have focused on the experimental aspect, and theoretical research [7, 15, 41,54] has received more attention recently. However, it is necessary to identify the effect of each of the parameters that define the texturing pattern. Therefore, it is important to identify the most important parameters influencing the frictional behaviour of lubricated contacts. By presenting the results as a Stribeck curve, performance of the lubricated contact as a function of operating conditions such as velocity, and texturing parameters such as texture depth, size, and density can be analyzed.

In some applications, the solid surfaces are so close together that asperities come into contact (see Fig. 3.1). Under these conditions, friction is determined predominantly by the interaction between the solids at asperity level where the boundary layers are being sheared when sliding. The bulk flow properties of the liquid play little or no part in the friction force when operating in the boundary lubrication regime. Boundary lubrication usually occurs under high-load and low-velocity conditions in machine components. 


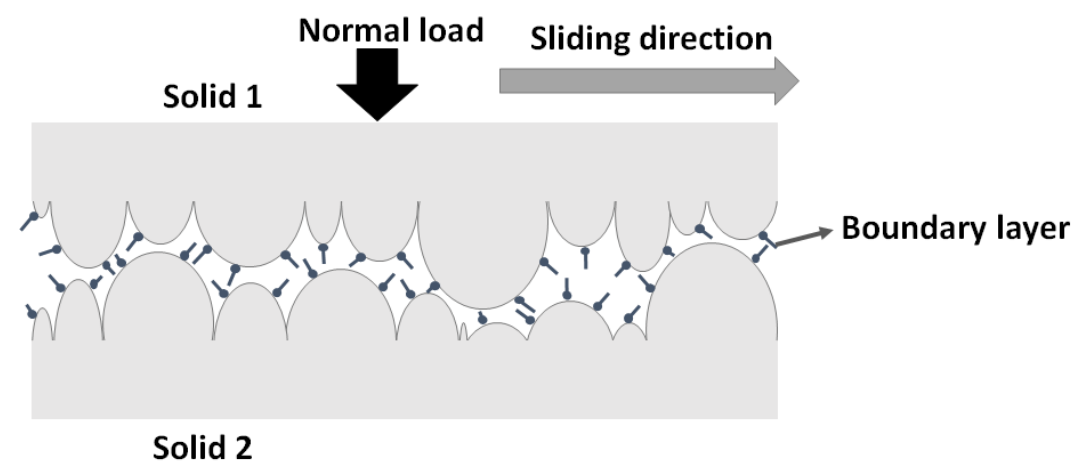

Figure 3.1. Schematic representation of two surfaces in contact [80].

Most machine components, such as gears and bearings, are likely to operate in the mixed lubrication regime where asperity contacts exist and the fluid contributes significantly to carrying the load. Modelling friction under mixed lubrication conditions is a challenging task; much effort has been devoted to this matter in the past few decades [7, 81-83]. There are two types of mixed lubrication models, based on statistic and deterministic contact models. The statistic contact model uses selected statistical parameters to represent random characteristics of surface roughness. A major shortcoming of these models is their inability to provide detailed information about local affairs, which have an influence on the mechanisms of lubrication and friction. The deterministic contact model using deterministic information of the surface roughness, is another common approach to simulate the behaviour of contacting asperities in mixed lubrication.

In this chapter, the deterministic asperity contact model is employed. Furthermore, the effect of surface texturing on the Stribeck curve, i.e. friction in lubricated contacts; in particular, the lubrication regimes hydrodynamic lubrication and mixed lubrication are studied numerically.

\subsubsection{Mathematical Modelling and Methods}

Greenwood and Williamson, in their contact model [84], assumed that the asperities are spheres with equal radius, and they employed a Gaussian distribution of the summit heights. However, in reality, the asperity radius is not constant for all asperities in contact. Also, most of the surfaces have a height distribution, which is not Gaussian. Although this model is quite reliable when the aforementioned conditions are applied, the assumption of a Gaussian distribution of equal summits is a drawback in this model. Here, in order to avoid Greenwood and Williamson's assumptions, a deterministic contact model was developed based on measured roughness data (see Fig. 3.2).

\section{mean line of the surface heights}

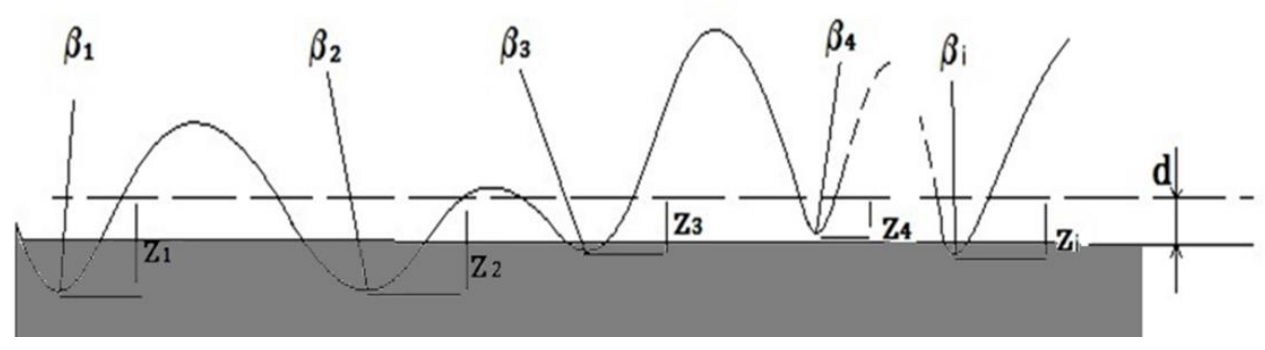

Figure 3.2. The contact between a rough surface and a flat surface [80]. 
Figure 3.2, shows a schematic illustration of the contact between a rough surface and a flat surface. By adding together the local contributions, the quantities of the real contact area and the number of asperities, the total force carried by the contact can be calculated deterministically, when the separation between the two surfaces $(d)$, is known. In this contact model, it is assumed that the asperities are deforming independently of each other, and that they have elliptical shapes. Furthermore, the radii of curvature are not equal for all asperities (the asperity radii are measured in sliding and in perpendicular direction $\left.\beta_{x i}, \beta_{y i}\right)$. The indentation of an asperity is:

$$
w_{i}=z_{i}-d
$$

In this equation, $z_{i}$ is the individual summit height and $d$ is the separation.

By determining the value of each deformed asperity $\left(w_{i}\right)$ and the total real contact area, and by adding the individual components of each asperity it is possible to calculate the load carried by the asperities $\left(F_{\mathrm{c}}\right)$. According to Johnson et al. [85], the total normal load on the contact in the ML regime is carried by the $\mathrm{BL}$ $\left(F_{\mathrm{C}}\right)$ and the $\mathrm{HL}\left(F_{H}\right)$ force component, therefore:

$$
F_{\mathrm{T}}=F_{\mathrm{C}}+F_{\mathrm{H}}
$$

Based on (Eq. 3.2), coefficients $\gamma_{1}$ and $\gamma_{2}$ are introduced:

$$
\gamma_{1}=\frac{F_{\mathrm{T}}}{F_{\mathrm{H}}}, \gamma_{2}=\frac{F_{\mathrm{T}}}{F_{\mathrm{C}}}
$$

The two coefficients, $\gamma_{1}$ and $\gamma_{2}$, refer to the $\mathrm{HL}$ component and the BL component, respectively. The two coefficients $\left(\gamma_{1}\right.$ and $\left.\gamma_{2}\right)$ are mutually dependent through the equation:

$$
1=\frac{1}{\gamma_{1}}+\frac{1}{\gamma_{2}}
$$

In the deterministic asperity contact model, by using the two coefficients $\gamma_{1}$ and $\gamma_{2}$ for the contact between a rigid flat surface against a rough one and the $\mathrm{HL}$ theory, the entire Stribeck curve can be calculated $[80,86]$.

In mixed lubricated contacts, in order to determine the frictional behaviour and to calculate the coefficient of friction of the contact, three parameters must be calculated: the load carried by the asperities, the load carried by the fluid, and the lubricant film thickness. Three equations are used to calculate the values of the three aforementioned parameters.

i- $\quad$ The first equation is Equation (3.2) i.e. $F_{T}=F_{C}+F_{H}$.

ii- $\quad$ The second equation is the film thickness relation (Eq. 1.6). In this study, the film thickness calculation is based on solving the Reynolds equation for textured surfaces [48].

iii- $\quad$ The third equation is the force carried by elliptical asperities:

$$
F_{\mathrm{c}}=\sum_{i=1}^{N} \frac{2}{3} E^{\prime} \beta_{i}^{\frac{1}{2}}\left(z_{i}-d\right)^{\frac{3}{2}}
$$


in which $E^{\prime}$ is the combined elasticity modulus and $\beta_{i}$ is the combined summit radius. The reduced elastic modulus and combined summit radius are given by:

$$
\frac{2}{E^{\prime}}=\frac{1-v_{1}^{2}}{E_{1}}+\frac{1-v_{2}^{2}}{E_{2}}, \beta_{i}=\sqrt{\beta_{x i} \cdot \beta_{y i}}
$$

where $E_{1}=E_{2}=E$ and $v_{1}=v_{2}=v$.

Finally, the total friction force $\left(F_{f}\right)$ in the $\mathrm{ML}$ regime is the sum of the friction force of the contacting asperities and the shear force of the lubricant $\left(F_{f H}\right)$ :

$$
F_{f}=\sum_{i=1}^{N} \iint_{A_{c i}} \tau_{c i} d A_{c i}+F_{f H}
$$

where the first term is the friction force due to contacting asperities; $N$ is the number of contacting asperities; $A_{c i}$ is the area of contact of a single asperity; $\tau_{c i}$ is the shear stress at asperity contact. For the $\mathrm{HL}$ component, the friction force can be calculated as:

$$
F_{f H}=\tau_{H} A_{H}
$$

where $A_{H}$ is the contact area of the hydrodynamic component and $\tau_{H}$ is the shear stress of the lubricant. For the contacting asperities, the friction is assumed to be of the Coulomb type, i.e.:

$$
\mu_{C i}=\frac{\tau_{c i}}{p_{c i}}
$$

with $p_{c i}$ being the average contact pressure on the current asperity. The coefficient of friction $\mu_{c i}$ is assumed constant for all asperities; therefore, the first term of Equation (3.10), can be written as:

$$
\sum_{i=1}^{N} \iint_{A_{c i}} \mu_{c} P_{c i} d A_{c i}=\mu_{C} F_{C}
$$

where the value of $\mu_{C}$ is experimentally determined and set in this work at 0.1 . Now, the coefficient of friction can be written as:

$$
\mu=\frac{F_{f}}{F_{N}}=\frac{\mu_{C} F_{C}+F_{f H}}{F_{N}}
$$

By solving this system of equations, the load carried by the asperities, the load carried by the fluid, and the separation are obtained and therefore the coefficient of friction in the ML regime can be calculated. The calculation of coefficient of friction in the ML regime is presented in the flow chart below, (see Fig. 3.3) 


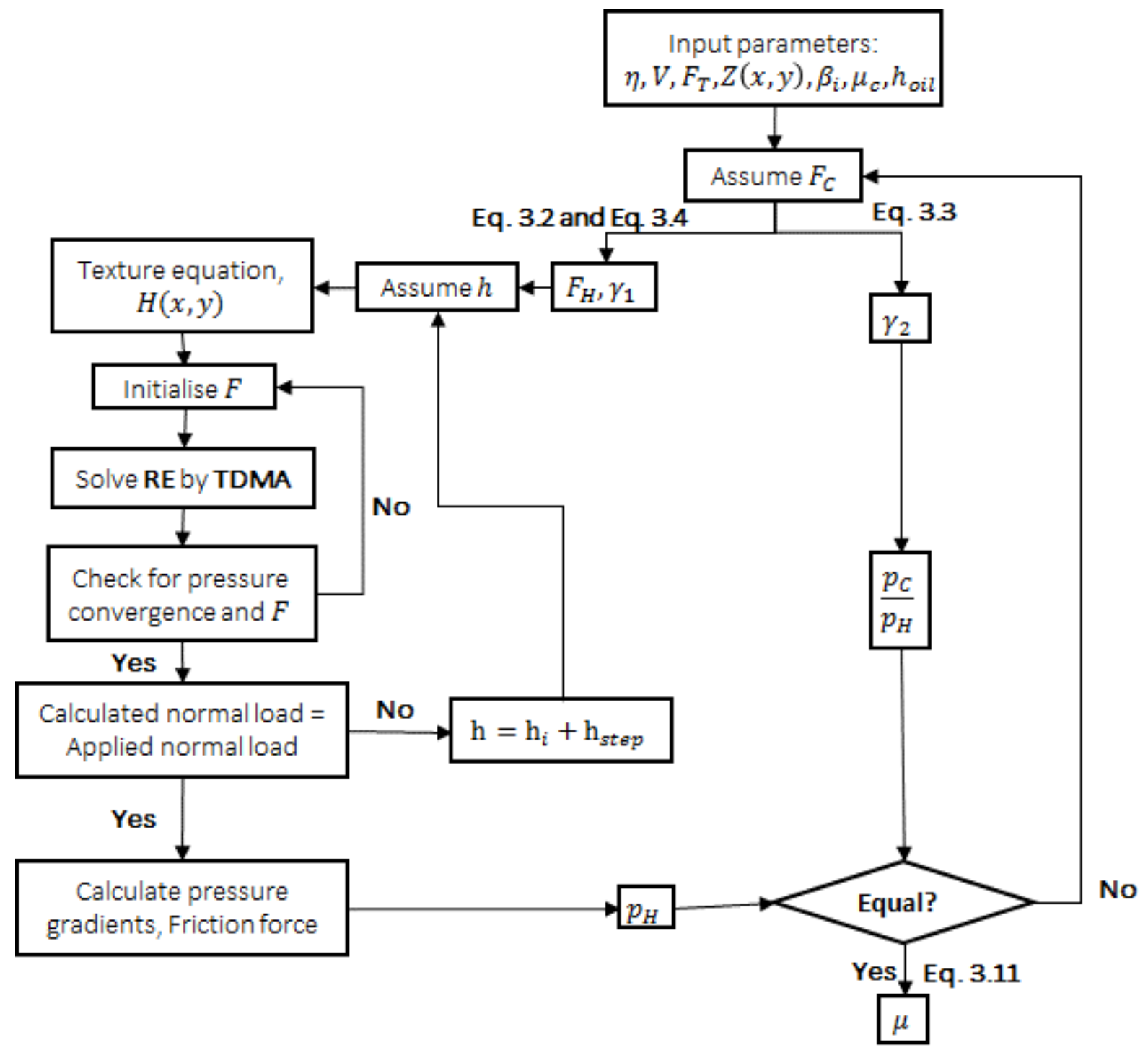

Figure 3.3. Mixed lubrication model algorithm [80].

Several simulations were carried out so as to study the effect surface and to compare the measurements results with the numerical calculations. More information on the influence of surface texturing on friction can be found in paper $\mathrm{C}$.

\subsubsection{Problem definition and its solution}

In these calculations, the number of pockets and the distance between them are combined by using the pitch parameter $\left(P_{x}\right)$ (see section 2.2.2). 


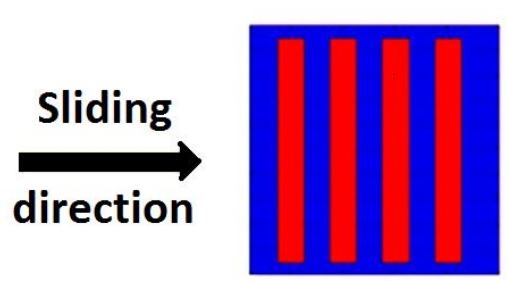

(a)

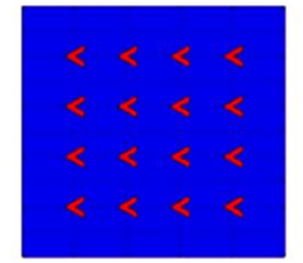

(b)
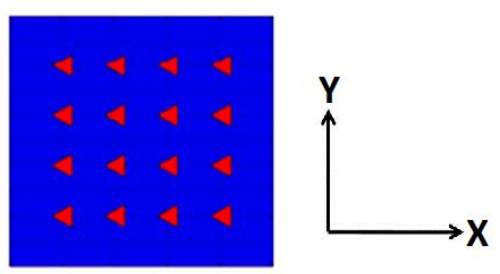

(c)

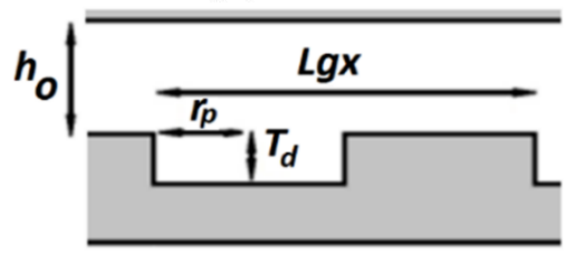

(d)

Figure 3.4. Schematic illustration of different patterns: a) grooves, b) chevrons, c) triangular pockets and d) schematic illustration of cavity profile [48].

From the previous study on the film thickness [48] it is possible to limit the investigation on texturing parameters and patterns. The effect of the cavity cross-sectional shape is studied [48] and the rectangular cross-section has been found as the more efficient pattern, therefore in the present study the calculations will be limited to cavities with a rectangular cross-section (see Fig. 3.4.d). To find the most efficient pattern the coefficient of friction calculation will be based on the linear groove, chevron and triangular pockets. The effect of different texturing parameters like cavity depth and cavity size as well as pitch is investigated. The texturing properties that are applied in this calculation are presented in (Table 3.1). The roughness measurement and surface topography analyses have been performed using a Keyence Color 3D LASER Scanning Microscope. In the appendix A surface roughness data is given and the reader is referred to this appendix for roughness details. The data in appendix $A$ is used in the calculations throughout the thesis.

Table 3.1. Texturing and roughness properties.

\begin{tabular}{ll}
\hline Parameter & Value \\
\hline Texture depth, $T_{d}$ & $10 \mu \mathrm{m}$ \\
Texture pitch, $P_{x}$ & 0.4 \\
Cavity size, $\mathrm{S}$ & $50 \mu \mathrm{m}$ \\
Normal load & $5 N$ \\
Average contact pressure & $0.05 \mathrm{MPa}$ \\
Dynamic viscosity & $8 \mathrm{mPa} \cdot \mathrm{s}$ \\
Elasticity modulus, $E$ & $210 \mathrm{GPa}$ \\
Poisson's ratio, $v$ & 0.3 \\
$R_{a}$ & $0.11 \mu \mathrm{m}$ \\
Roughness & See appendix A \\
\hline
\end{tabular}

\subsubsection{Comparison of patterns}

To determine the effect of texturing of lubricated contacts on friction, several simulations were carried out using different texturing parameters. The coefficient of friction for grooves as well as chevron and triangular pockets parallel to the moving direction has been calculated. From a previous study [48], simulations indicated that a higher lubricant pressure generation is achievable by closed grooves, because the side leakage for open grooves is higher than for closed grooves; therefore, in this study closed grooves were chosen. 


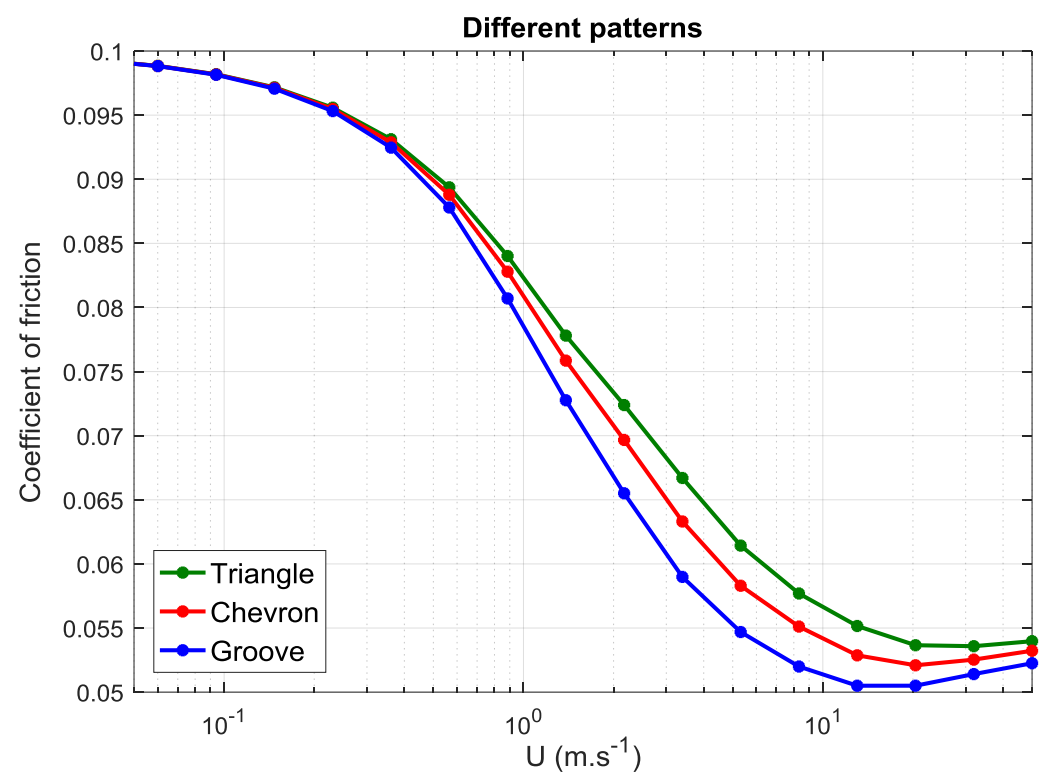

Figure 3.5. Comparison between different patterns.

In Fig. 3.5 the calculated coefficient of friction as a function of the velocity for the different patterns is presented. In the boundary lubrication region, the normal load is carried solely by the interacting asperities. In this region the coefficient of friction is high due to the roughness of the interacting surfaces, and as a result the different texturing patterns have almost the same value as there is limited hydrodynamic action.

By increasing the velocity, the wedge effect caused by the existence of textures at the surface leads to an increase in the lubricant pressure. The pressure generation in the fluid film results in a lower number of asperities in contact, and a drop in the coefficient of friction. In this region, the load over the contact is carried by the lubricant as well as solid asperities; therefore, the performance of the lubricant film in contact has an influential effect on the coefficient of friction. In the mixed lubrication regime, patterns that are more successful in film formation can cause a higher reduction in friction. The biggest drop in the coefficient of friction in this region belongs to the surfaces with a groove pattern and, after that, the chevrons are most successful to reduce friction.

In the higher velocity range, the operational lubrication regime shifts from the mixed to the hydrodynamic lubrication regime. In the hydrodynamic lubrication regime, by increasing the sliding velocity the shear stress also increases, and a higher shear stress results in a higher coefficient of friction. For patterns, that are more efficient in film formation, the transition point between the mixed and hydrodynamic lubrication regime occurs at a lower velocity.

\subsubsection{Effect of geometrical properties}

The coefficient of friction in lubricated contacts in the ML and $\mathrm{HL}$ regimes is influenced by several parameters, such as lubricant viscosity and contact pressure. When these parameters have a fixed value it is possible, by changing the geometrical parameters, to detect the influence of the size of the surface texturing on the frictional behaviour of the contact. In this subsection an optimization of the geometrical parameters is performed. In this optimization, the effect of texture pitch, cavity size, and texture depth on the coefficient of friction for cavities with a rectangular profile (Fig. 3.4) is studied. As mentioned in chapter 2 , grooves can be characterised by three geometry parameters, and in the case of chevrons, a fourth parameter is needed to define this pattern, i.e. the cavity width ratio $(K)$. 


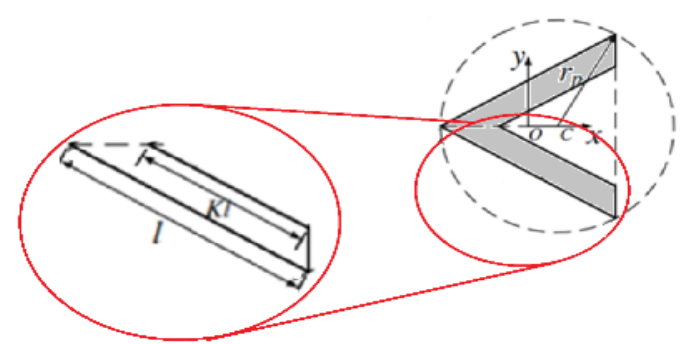

Figure 3.6. Definition of cavity width ratio, $K$, is the ratio of the inner wall length to the outer wall length [48].

In the calculations presented, cavity width ratio $(K)$ the ratio of the inner wall length to outer wall length, is constant and equal to 0.5 .

\section{- The effect of texture depth $\left(T_{d}\right)$}

In Fig. 3.7, simulation results are shown for the coefficient of friction as a function of velocity by varying the texture depth for the groove and chevron pattern. When the depth is tending to become zero, there is no film formation because of the absence of the wedge effect in the flat-flat contact and the lack of texturing.

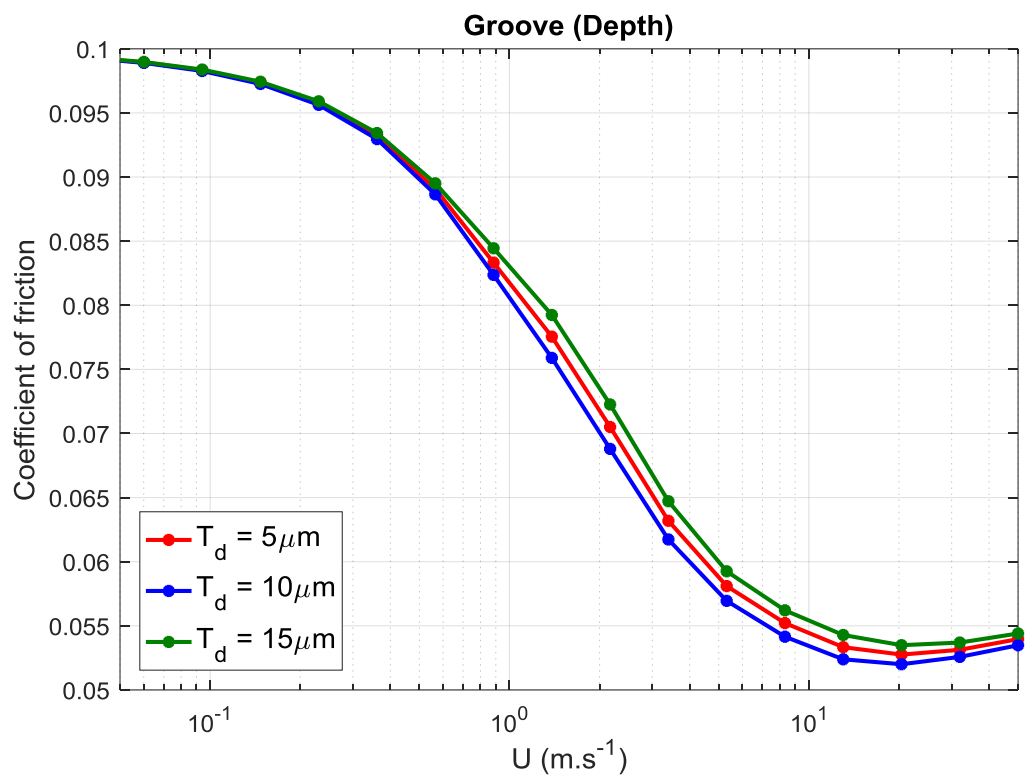

(a) 


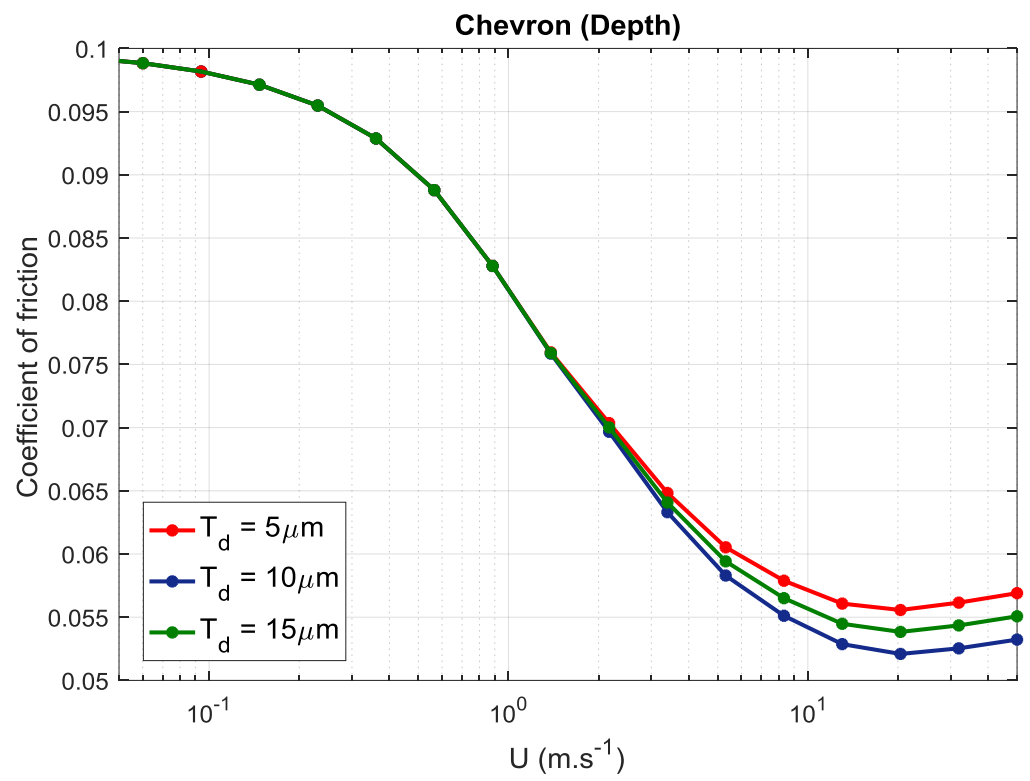

(b)

Figure 3.7. Coefficient of friction as a function of velocity for patterns with different texture depth for:

(a) grooves and (b) chevrons.

For both chevron and groove patterns, the fluctuation of texture depth affects the hydrodynamic lubrication component in mixed lubrication. A texture depth that is close to $10 \mu \mathrm{m}$ leads to a reduction in the coefficient of friction due to a film thickness increase. After the texture depth passes the optimum value for the texture depth, i.e. $10 \mu \mathrm{m}$, deeper cavities result in an increase in the coefficient of friction. This phenomenon is caused by the reduction in lubricant film thickness [48]. From Fig. 3.7, it is concluded that when the textured contact is operating near the transition $\mathrm{ML}$ and $\mathrm{HL}$ regime the texture depth has an influence on the frictional behaviour.

\section{- $\quad$ The effect of cavity size (S)}

In Fig. 3.8, the effect of the cavity size $(S)$ of the chevron and groove patterns on the coefficient of friction is shown. As found for the texturing depth, an optimum value for the cavity size $(S)$ exists. For sizes tending to zero, or which are very large, there is almost no pressure generation, due to not enough wedge effect. 


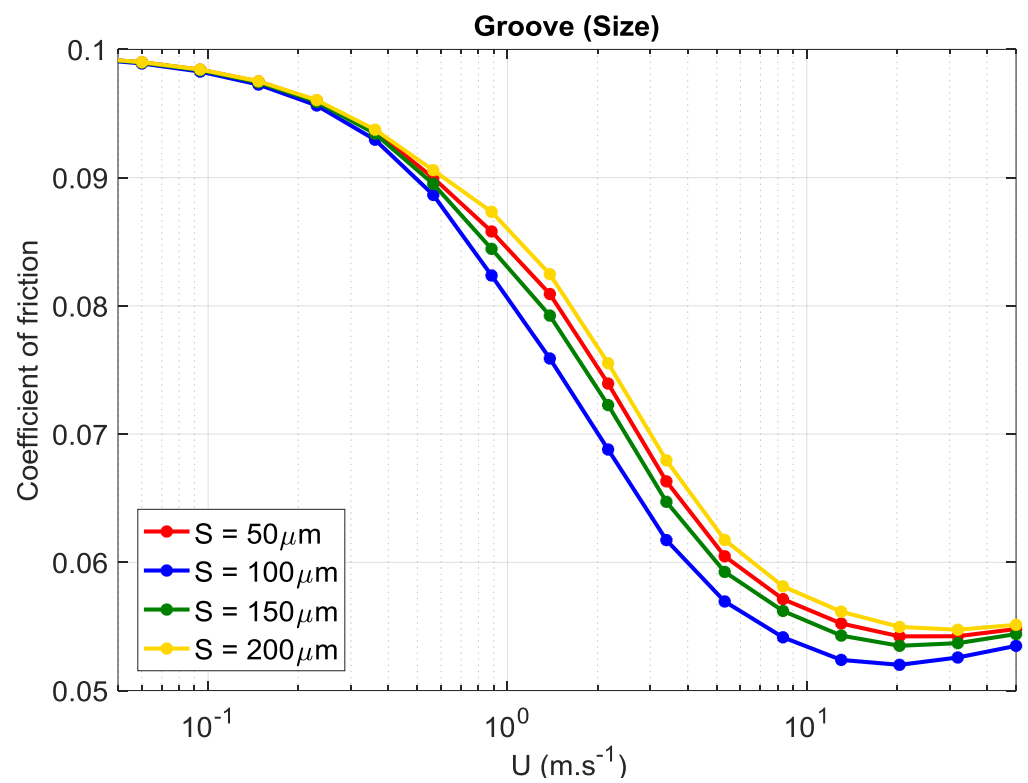

(a)

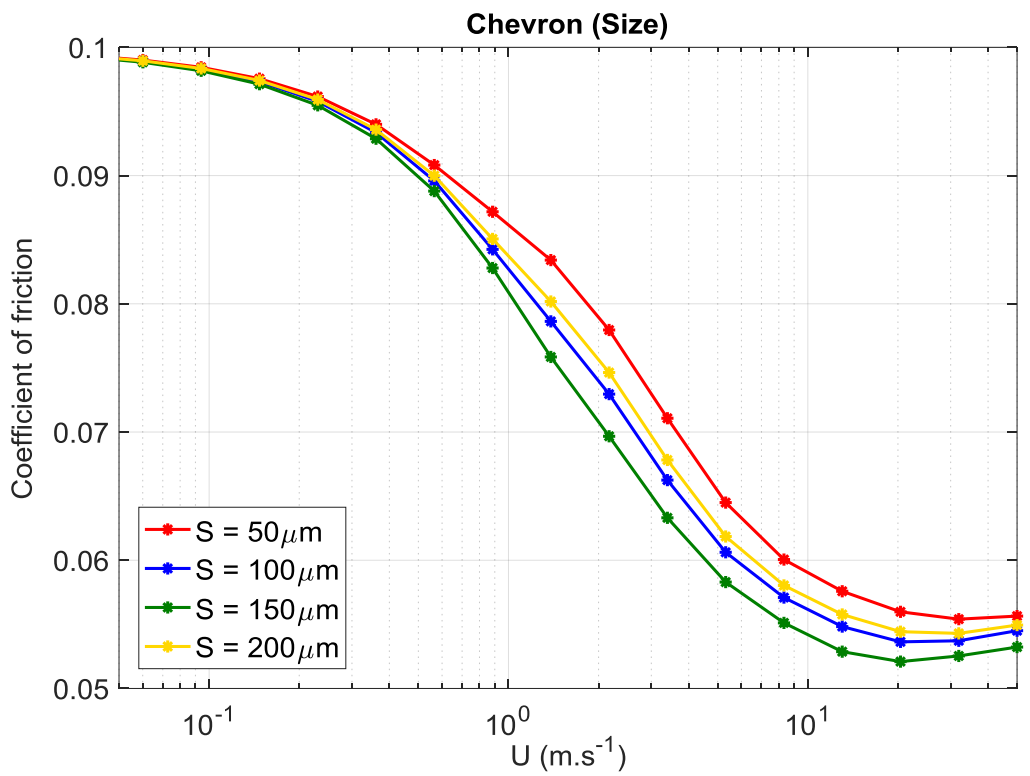

(b)

Figure 3.8. Coefficient of friction as a function of velocity for patterns with different cavity sizes $(S)$ for: (a) grooves and (b) chevrons.

Changing the cavity size will affect the hydrodynamic lubrication component. In the case of grooves (see Fig. 3.8.a), by increasing the cavity size to $100 \mu \mathrm{m}$, the coefficient of friction is reduced due to the increase in film thickness. After passing the $S=100 \mu \mathrm{m}$, the larger cavities cause a drop in film thickness, which causes an increase in the coefficient of friction. For higher velocities, the hydrodynamic lubrication is the dominant lubrication mechanism. The lowest coefficient of friction is achievable when the size is approximately $100 \mu \mathrm{m}$. In the case of chevrons (see Fig. 3.8.b), as shown in the previous study [48], the optimum value for the film thickness is achievable when the size of the chevron cavity is around $150 \mu \mathrm{m}$. Therefore, the lowest coefficient of friction can be obtained when cavities with $S=150 \mu \mathrm{m}$ are applied. 


\subsubsection{Conclusion}

The aim of these calculations was to study and predict the effect of surface texturing on friction for parallel sliding contacts. In order to obtain a better understanding of the friction phenomenon, a numerical model has been developed. The effect of several parameters on the frictional behaviour of lubricated parallel siding contacts such as pattern type, depth and size have been studied. The results can be summarized as:

- Of the patterns studied, the groove pattern shows the lowest coefficient of friction. When the distances between texture cells are the same, i.e. the pitch is constant, the groove pattern is more successful in generating load-carrying capacity, due to higher textured area fraction per unit area. Chevrons are more beneficial than the triangular pockets.

- For both chevron and groove patterns, the texturing parameters such as depth and size values have an influence on the hydrodynamic lubrication component and therefore also in the mixed lubrication regime.

- For different patterns, increase in the depth of cavities to a certain value leads to the reduction of the coefficient of friction due to the growth in film thickness.

- In case of groove pattern, the optimum cavity size is approximately $S=100 \mu \mathrm{m}$ and in the case of chevrons, the optimum cavity size is approximately $S=150 \mu m$.

- The optimum depth for the different patterns is approximately $10 \mu \mathrm{m}$.

\subsection{Surface texturing on frictional behaviour under starved lubrication condition}

As mentioned in section 2.3, the amount of lubricant supplied to the contact can be insufficient in this case, one may speak of starved lubrication. Therefore, it is of interest to identify the most important parameters influencing the frictional behaviour of the lubricated contacts under starved lubricated condition too. Although in literature a lot of attention is paid to the influence of starvation on the full film lubrication, little work is done on mixed lubrication and even fewer investigations have been made with respect to the frictional behaviour of starved lubricated textured surfaces.

In starved lubrication condition, when the supply of lubricant in the contact is limited, a correction on the film thickness formula is necessary. Therefore, in this section, by combining this modified film thickness with the mixed lubrication model, a modified mixed lubrication model for starved contacts is obtained. Moreover, the consequences of starvation on friction depicted in the Stribeck curve due to the change in film formation is discussed. To model the asperity contact pressure in mixed lubrication, the deterministic mixed lubrication model is employed. By using this model the friction as a function of the operating conditions like velocity and the texturing parameters texture depth, size and texture density can be studied.

In this section, the effect of surface texturing on the Stribeck curve is investigated numerically. The influence of starvation on the film thickness is discussed in the previous section and in paper B [51]. A more comprehensive study of starvation on the frictional behaviour of sliding contacts is presented in paper D.

\subsubsection{Mathematical solution and modelling}

To study the effect of starvation on the frictional behaviour of the textured surfaces with different texturing patterns and parameters, several simulations are carried out. The optimal depth and size for the chevron and grooved patterns are calculated, as it is presented in section 2.2.2.

\subsubsection{Problem definition and its solution}


The limited amount of lubricant in the inlet region of the contact $\left(h_{\text {oil }}\right.$, see Fig. 2.8$)$ affect the film thickness for textured surfaces as shown in section 2.3. In order to have a better understanding of starved lubrication calculations with respect to influence of input film thickness and texturing parameters like, texture pitch $\left(P_{x}\right)$, texture depth $\left(T_{d}\right)$ and texture size $(S)$ on the coefficient of friction simulations are carried out for the linear groove and chevron patterns.

From the previous study on film thickness [48], it is possible to limit the investigation on texturing parameters and patterns. Therefore, in the present section the calculations will be limited to the rectangular cross sectional cavities (see Fig. 3.4.d). Here, linear grooved and chevrons will be analyzed. The operating conditions used in the calculations are given in (Table 3.2). The roughness data is the same to the data in the previous section $R_{a}=0.11 \mu \mathrm{m}$.

Table 3.2. Operating conditions.

\begin{tabular}{ll}
\hline Normal load & $5 N$ \\
Average contact pressure & $0.05 \mathrm{MPa}$ \\
Contact area & $10^{-4} \mathrm{~m}^{2}$ \\
Lubricant viscosity & $8 \mathrm{mPa} . \mathrm{s}$ \\
\hline
\end{tabular}

In these calculations the cavity width ratio $K$ is kept constant and equal to 0.5 . The coefficient of friction in the $\mathrm{ML}$ regime for starved lubricated contacts can be calculated as expressed in the chart presented below, see Fig. 3.9.

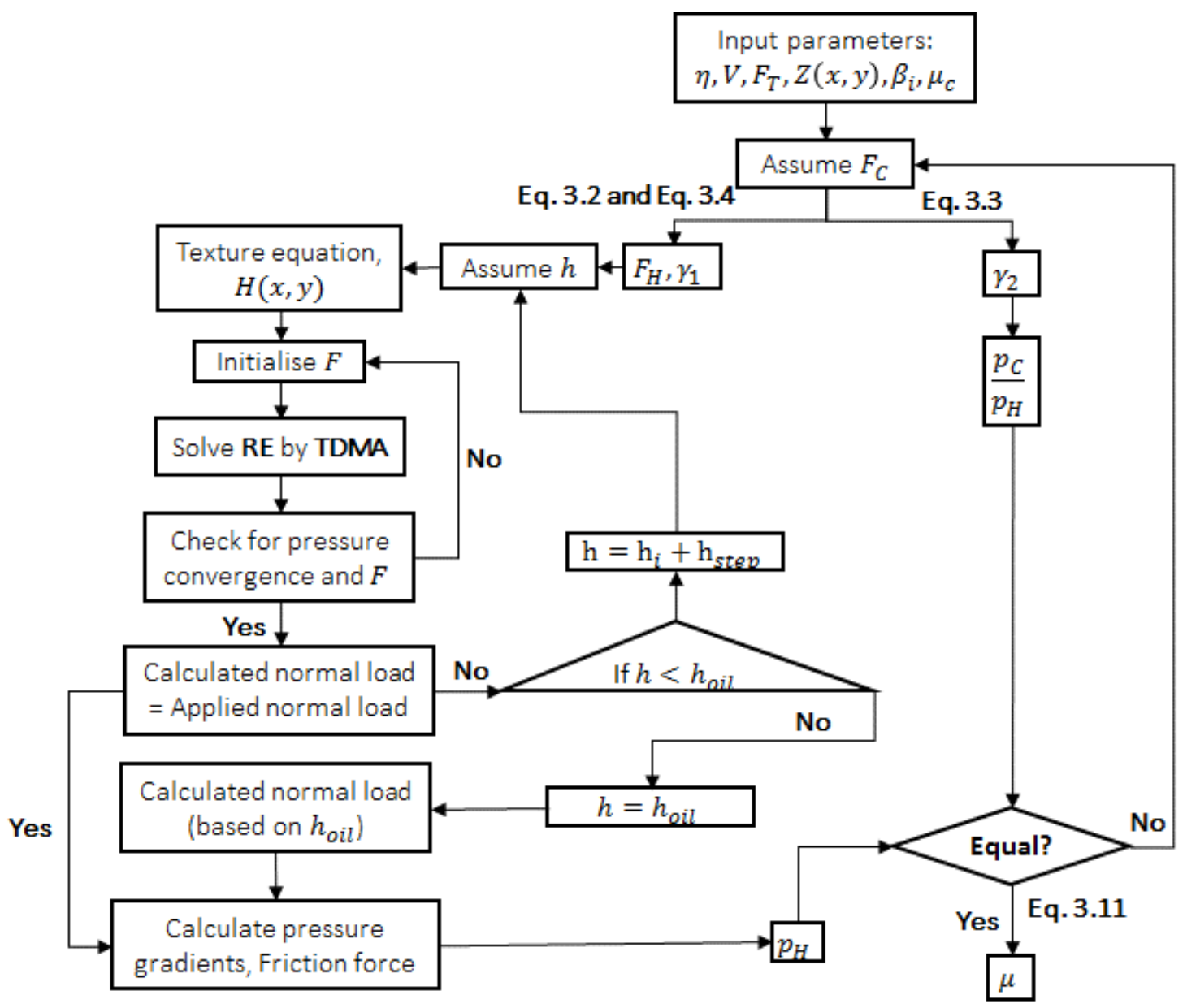

Figure 3.9. Algorithm mixed lubrication model for starved lubrication. 


\subsubsection{The effect of texture depth $\left(\boldsymbol{T}_{\boldsymbol{d}}\right)$}

The coefficient of friction is calculated based on three different values of texture depth, $5 \mu \mathrm{m}, 10 \mu \mathrm{m}$ and $15 \mu \mathrm{m}$. In Fig. 3.10 and Fig. 3.11, the simulation results show the effect of different values for texture depth based on starved and non-starved lubrication for grooves and chevrons. In Fig. 3.10.a, it can be seen from the outcome that a depth of $10 \mu \mathrm{m}$ results in the lowest coefficient of friction. In Fig. 3.10.b, a limited input film thickness $h_{\text {oil }}=10 \mu \mathrm{m}$ is applied in order to simulate starvation. The results in Fig. 3.10.b show that the calculated coefficient of friction for different values of texture depth tend to the same values, therefore the Stribeck curves are merging together.

Table 3.3. Texture properties.

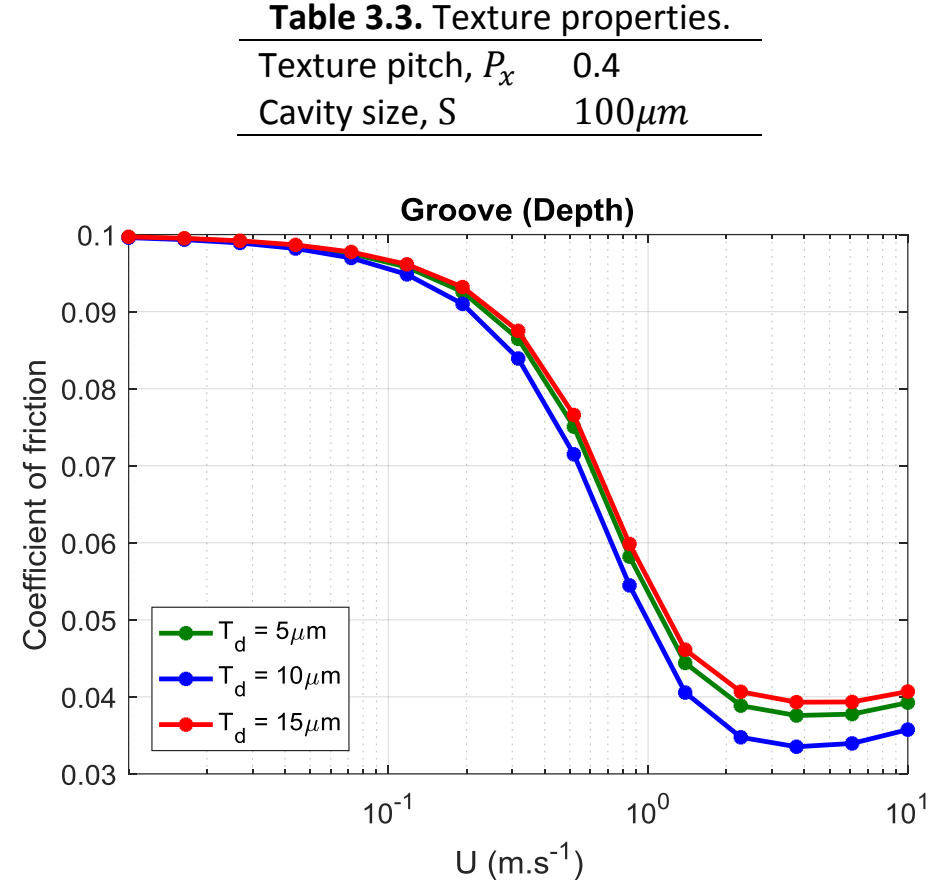

(a)

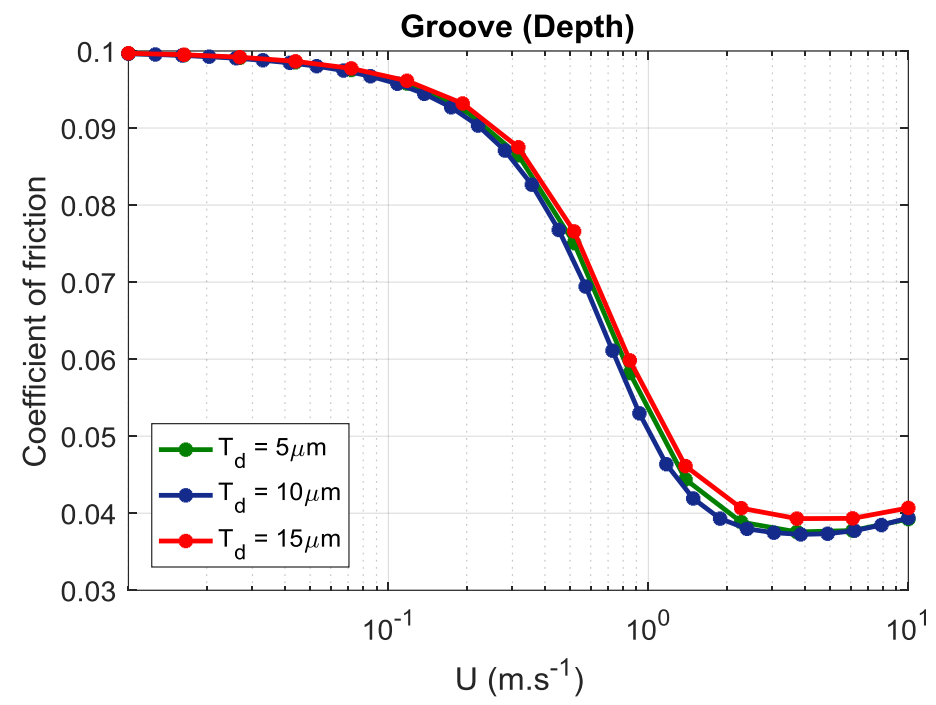

(b)

Figure 3.10. Stribeck curves as a function of texture depth for grooves when the lubrication is: a) nonstarved, b) starved and $h_{\text {oil }}=10 \mu \mathrm{m}$. 


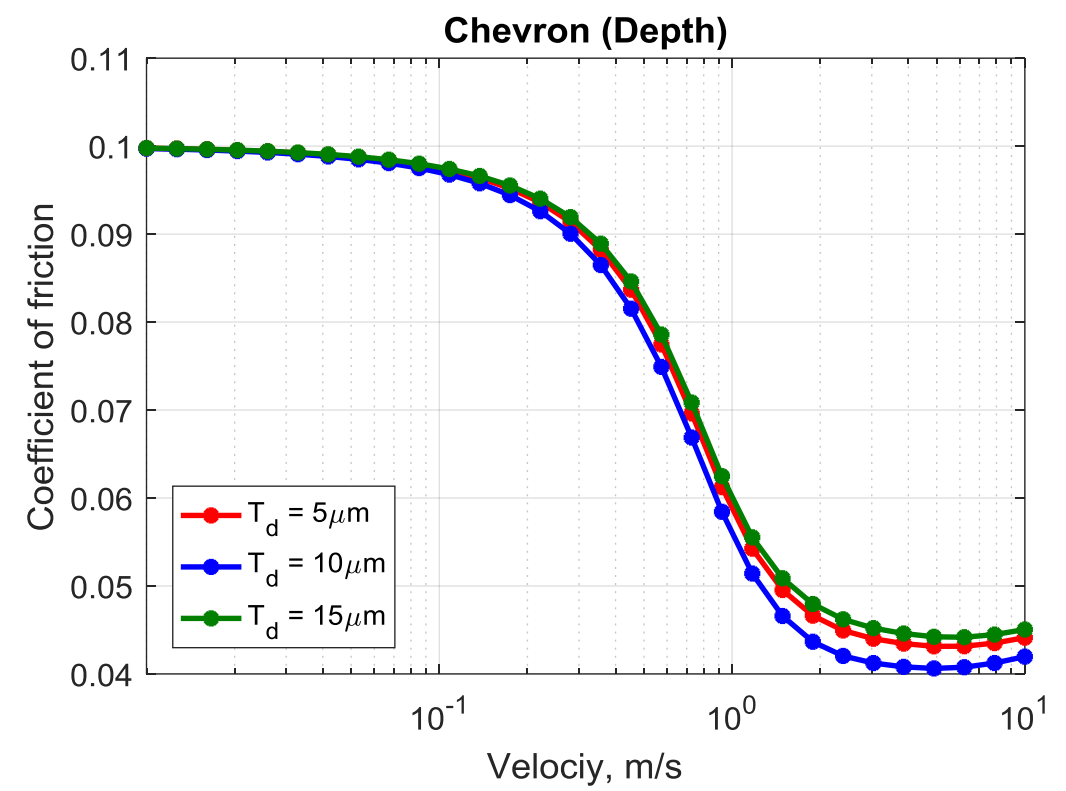

(a)

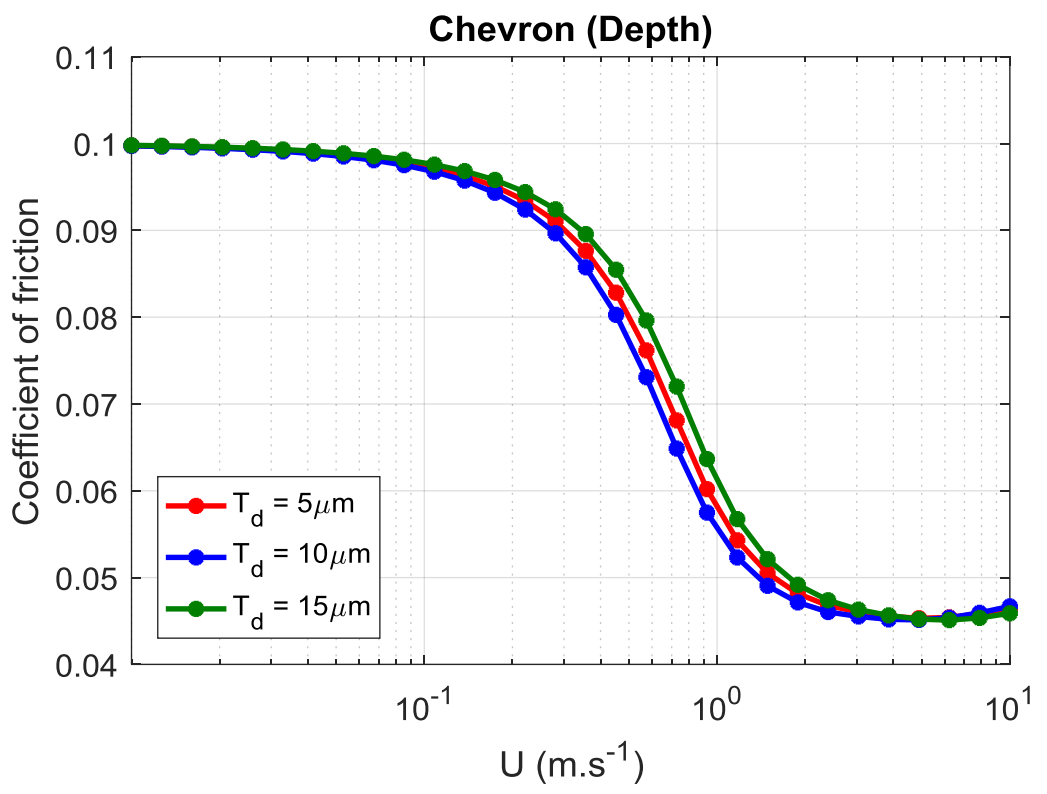

(b)

Figure 3.11. Stribeck curves as a function of texture depth for chevrons when the lubrication is: a) nonstarved, b) starved and $h_{\text {oil }}=10 \mu \mathrm{m}$.

In Fig. 3.11.a, in the case of the chevron pattern similar to the groove pattern, the lowest coefficient of friction in case of non-starved lubrication is achieved when the depth is around $10 \mu \mathrm{m}$. In Fig 3.11.b in the case of the starved lubrication, the results show that the calculated coefficients of friction for different values of texture depth are tending to the same values (Stribeck curves are merging together). By comparing the curves in Fig. 3.11, and based on the results from the study on the film thickness (paper B), which shows that a higher film thickness is achievable for grooves and chevrons when the depth is 
around $10 \mu \mathrm{m}$, it is possible to conclude that the lowest coefficient of friction is achievable when the film thickness has the highest value. This is because in case of mixed lubrication an increasing separation reduces asperity contact. Although the growth in depth of cavities to the optimum values leads to greater film thicknesses, in the case of starved lubrication this growth in lubricant film thickness is limited by the oil supply, resulting in a limitation of the effect of this parameter on the coefficient of friction, as shown in Fig. 3.11.

\subsubsection{The effect of texture size $(\boldsymbol{S})$}

In this section, to study the effect of size on the coefficient of friction for starved lubricated contacts the texture depth and pitch are set as constant and the size is changed. The coefficient of friction is calculated for three different values for the texture size parameter $(S)$. In Fig. 3.12 and Fig. 3.13, the simulation results show the effect of different values for the texture size for starved and non-starved lubrication of grooves and chevrons. In Fig. 3.12.a, in the case of the groove pattern with a size around $100 \mu m$, the friction force has the lowest value. In Fig. 3.12.b, a limited oil film thickness is applied to the contact in order to simulate starvation. The results in Fig. 3.12.b show that the calculated Stribeck curves for different values of texture size merge in case of starved lubrication for a texture depth of $10 \mu \mathrm{m}$.

Table 3.4. Texturing properties.

\begin{tabular}{ll}
\hline Texture depth, $T_{d}$ & $10 \mu \mathrm{m}$ \\
Texture pitch, $P_{x}$ & 0.4
\end{tabular}

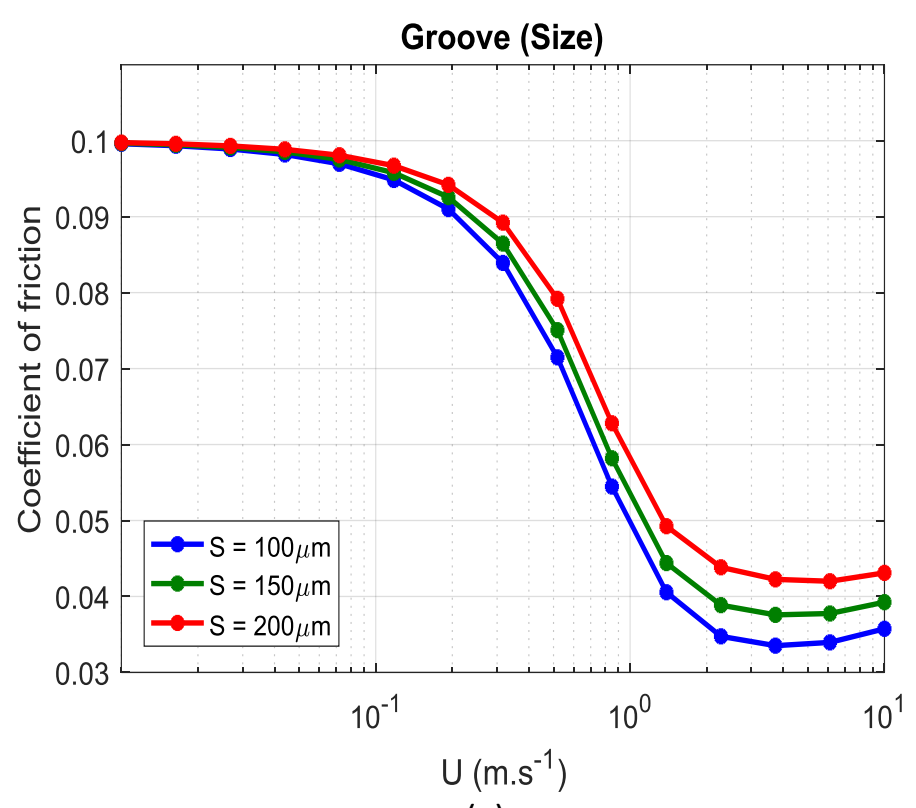

(a) 


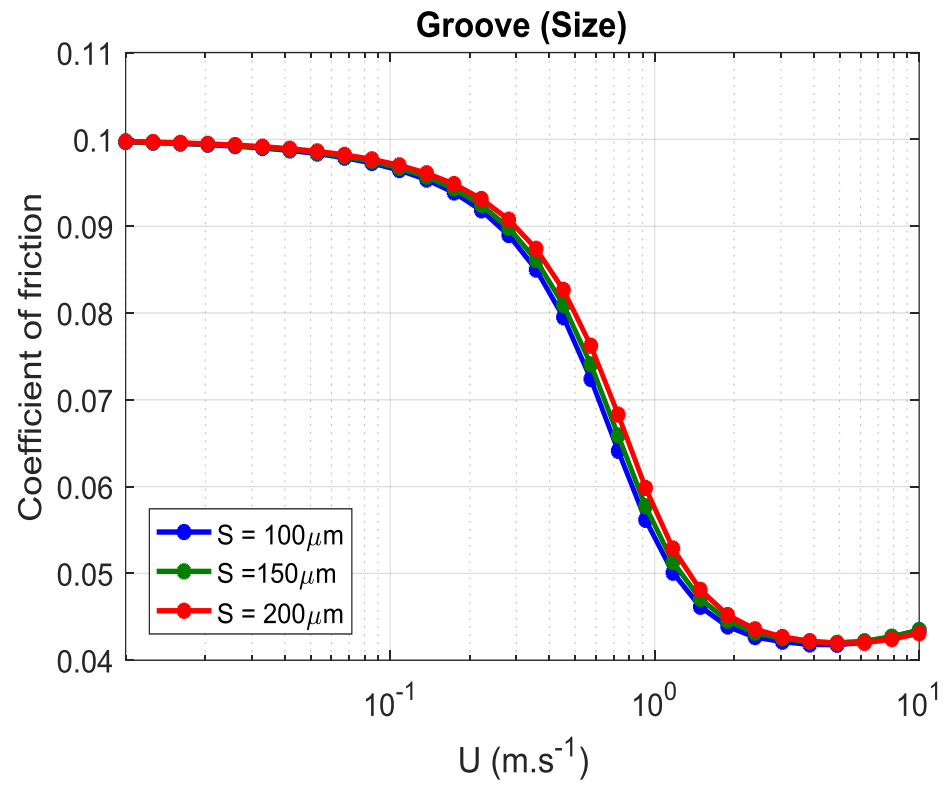

(b)

Figure 3.12. Stribeck curves as a function of cavity size $S$ for grooves when the lubrication is: a) nonstarved, b) starved and $h_{\text {oil }}=10 \mu \mathrm{m}$.

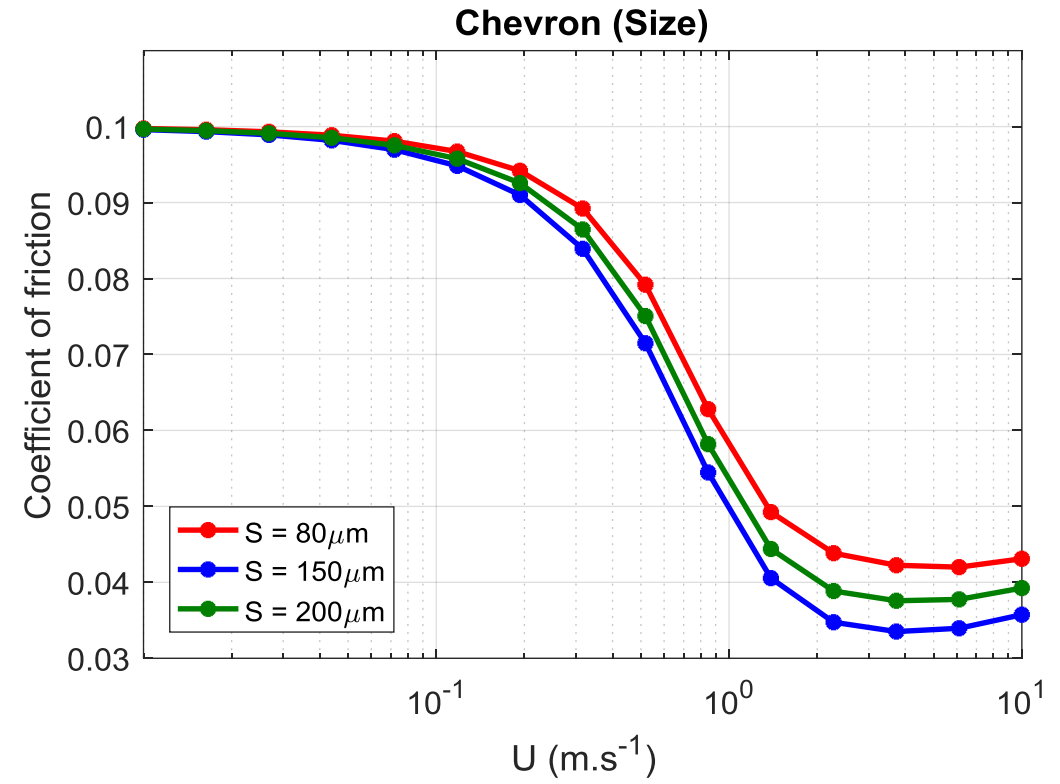

(a) 


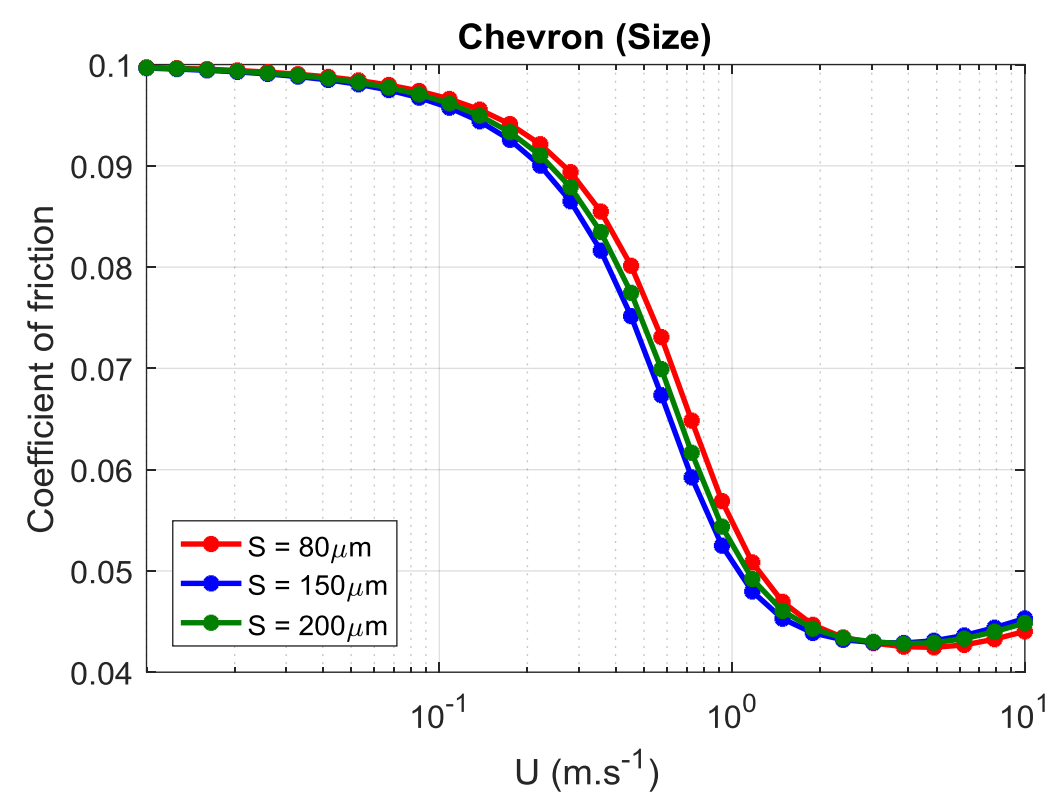

(b)

Figure 3.13. Stribeck curves as a function of cavity size for chevrons when the lubrication is: a) nonstarved, b) starved and $h_{\text {oil }}=10 \mu \mathrm{m}$.

In Fig. 3.13.a, where the chevron pattern similar to the groove pattern, the lowest coefficient of friction for non-starved lubrication is achievable when the size is around $150 \mu \mathrm{m}$. Also in this case, starved lubrication results in merging of the curves as is shown in Fig. 3.13. Changing the size parameters has an influence on coefficient of friction for the fully flooded contact but for the starved lubricated situation the effect of this parameter is limited.

\subsubsection{Effect of input film thickness on coefficient of friction}

In Fig. 3.14.a, the calculated film thickness and in Fig. 3.14.b, the calculated coefficient of friction for grooves, when $T_{d}=10 \mu \mathrm{m}, S=100 \mu \mathrm{m}$ and $P_{x}=0.4$, is shown. In these calculations, the effect of different input film thickness is studied. The coefficient of friction for four different input film thicknesses $h_{\text {oil }}=0.025,4,6$ and $8 \mu \mathrm{m}$ and the film thickness for three different input film thicknesses $h_{\text {oil }}=$ $0.5,4$ and $6 \mu \mathrm{m}$ are calculated.

Table 3.5. Texturing properties.

\begin{tabular}{ll}
\hline Texture depth, $T_{d}$ & $10 \mu \mathrm{m}$ \\
Texture pitch, $P_{x}$ & 0.4 \\
Cavity size, $\mathrm{S}$ & $100 \mu \mathrm{m}$ \\
Normal load & $5 \mathrm{~N}$ \\
Average contact pressure & $0.05 \mathrm{MPa}$ \\
Lubricant viscosity & $8 \mathrm{mPa} . \mathrm{S}$ \\
$R_{a}$ & $0.11 \mu \mathrm{m}$ \\
\hline
\end{tabular}




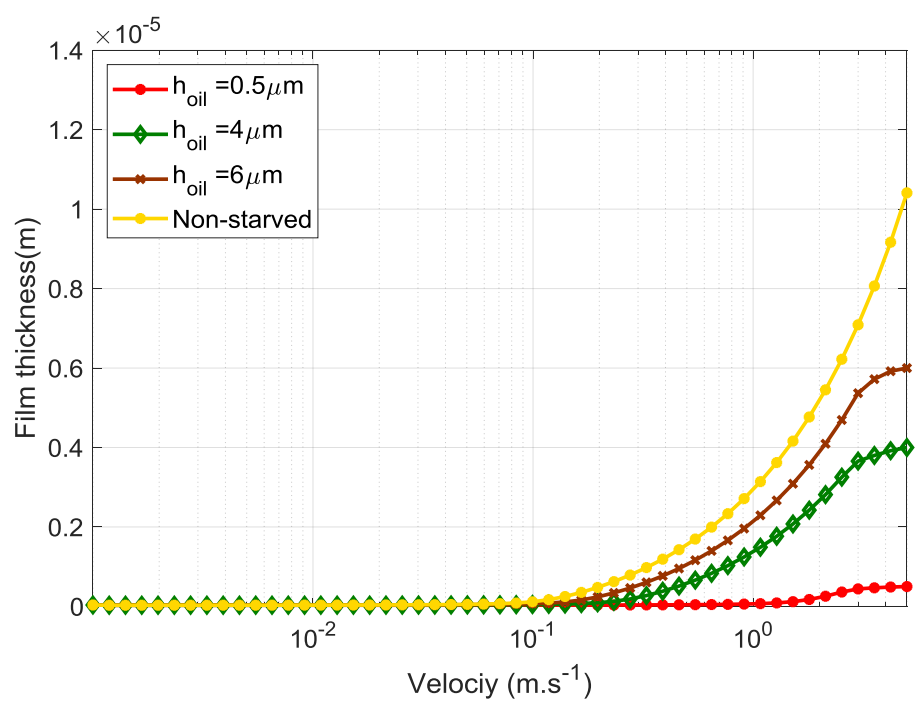

(a)

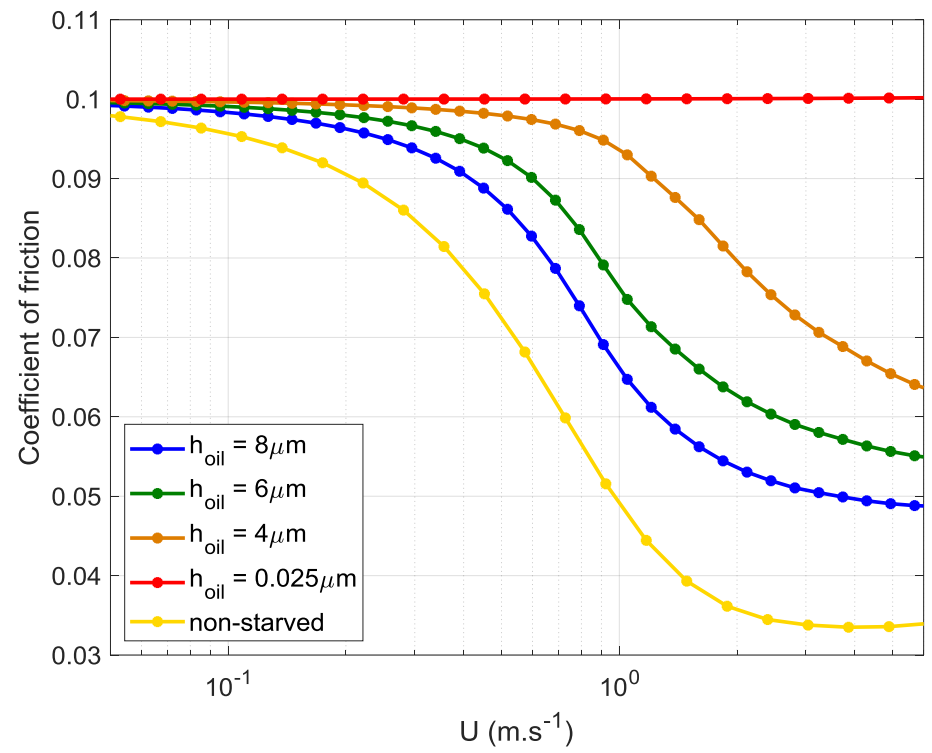

Figure 3.14. Effect of starvation on a) film thickness and b) coefficient of friction.

The results of the Stribeck curve and film thickness for different values of the oil thickness applied to the contact are presented in Fig. 3.14. The behaviour of the starved Stribeck curve and film thickness as a function of the applied oil thickness can be described as follows:

- When the input film thickness $\left(h_{\text {oil }}\right)$ is lower than $0.025 \mu m$, the Stribeck curve tends to transform into a straight line and the separation stays on the same level as in the BL regime, and the film thickness does not change in comparison with the boundary lubrication condition.

- If the $h_{o i l}$ varies between $0.025 \mu \mathrm{m}$ and $8 \mu \mathrm{m}$, the friction level in the $\mathrm{HL}$ and ML regimes starts to decrease and the film thickness increases. By reducing the value of applied oil layer thickness to the contact, the mixed lubrication regime becomes less steep in this range of applied oil film thickness. 


\subsubsection{Conclusion}

The goal was to study and predict the effect of surface texturing on the frictional behaviour for parallel sliding starved lubricated contacts. This model is a numerical model based on the Reynolds equation with the Elrod cavitation algorithm formulation. By combining the calculated film thickness with the deterministic asperity model, the coefficient of friction is calculated. The effect of several parameters on the frictional behaviour, such as depth, size, texture pitch and input oil film, has been studied.

In the case of starved lubrication, when the value of input film thickness $\left(h_{\text {oil }}\right)$ decreases the starvation effect gains a greater influence on the film thickness. As a result, the effect of variation of the texture parameters (depth and size) on the coefficient of friction also decreases.

\subsection{Summary}

In this chapter, in order to obtain a better understanding of the friction phenomenon, a numerical model is presented. The effect of several parameters on the frictional behaviour of lubricated parallel siding contacts, such as pattern type, depth and size, has been studied. Of the patterns studied, the groove pattern shows the lowest coefficient of friction. When the distances between texture cells are the same, i.e. the pitch is constant, the groove pattern is more successful than the other patterns in generating loadcarrying capacity, because of the higher textured area fraction per unit area. Chevrons are more beneficial than the triangular pockets. The growth of texture depth to the specific value of this parameter for different patterns leads to the reduction of the coefficient of friction due to an increase in film thickness. Further, the effect of starvation on the frictional behaviour of textured contacts is studied and the results are presented, as well as the effect of several parameters on starvation. 


\section{Chapter 4}

Experimental Validation of the Mixed Lubrication Model

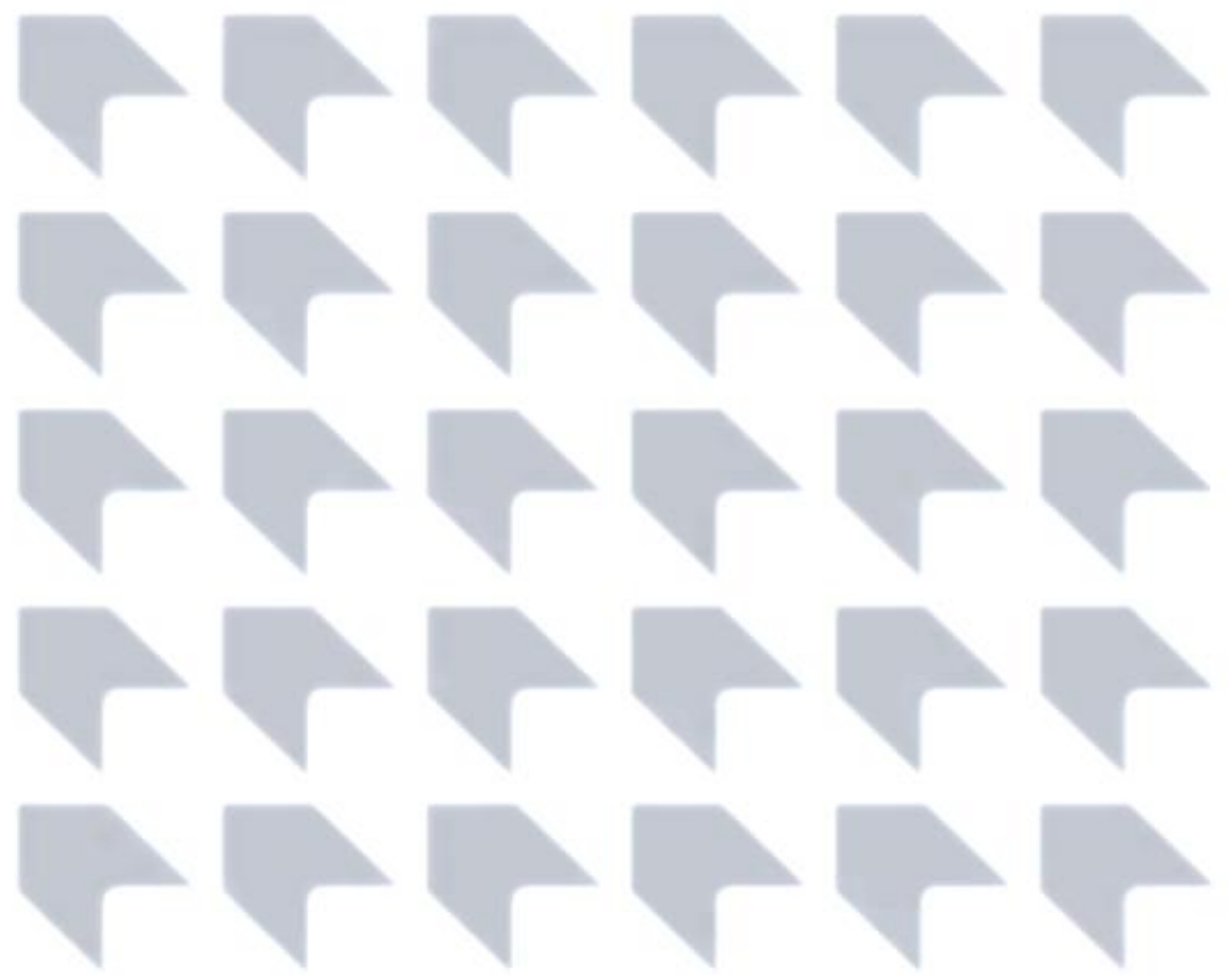




\section{Introduction}

In Chapter 3, the mixed lubrication model is presented with a deterministic representation of the rough surfaces. Further, the results of the friction calculation for different texturing parameters are presented. Chapter 4 describes the test setup on which experiments were performed in order to validate the mixed lubrication model. This setup is used to measure the friction force for different texturing patterns at different velocities in the case of parallel sliding contacts.

In section 4.1 of this chapter the test conditions are defined. In section 4.2 , the measurement setup is explained. In section 4.3, the results of the experimental measurements are presented and a comparison between numerical results and experimental measurements is explained and a parametric experimental investigation based on different texture properties is performed.

\subsection{Measurement condition}

To measure friction of lubricated plane parallel sliding textured surfaces and in order to validate the numerical model that is described in the previous chapter, a test setup and test method is used as described in this section. To study the frictional behaviour of contacts, a set of friction measurements is performed using a modified pin-on-disk tribometer. For these measurements "NS3 Shell" oil is used as lubricant. The viscosity of the oil is based on its commercial information. Furthermore, in order to study the effect of texturing on the Stribeck curve and to cover the lubrication regimes, two different loads are applied.

To measure the friction in a flat-on-flat situation, the most challenging obstacle is to prepare samples with respect to flatness and to align these flat surfaces. The existence of any misalignment or surface waviness of the samples will cause a measurement error. In Table 4.1, the operating conditions of the measurements are presented.

Table 4.1. Measurement conditions

\begin{tabular}{|l|l|}
\hline Normal load & $5,10 \mathrm{~N}$ \\
\hline Velocities & $0.05,0.1,0.2,0.3,0.4,0.5 \mathrm{~m} . \mathrm{s}^{-1}$ \\
\hline Lubricant type & NS3 Shell \\
\hline Lubricant viscosity & $32 \mathrm{mPa} . \mathrm{s}$ \\
\hline
\end{tabular}

\subsection{Setup}

\subsubsection{Pin-on-Disk}

The experiments were conducted in a pin-on-disk tribo-tester (CSEM), see Fig. 4.1; the tested specimens are flat textured steel blocks (the pin) pressed against rotating steel disks held in a cup filled with lubricant. The samples were prepared in the workshop of the University of Twente, the laser texturing of the samples was conducted at Philips and at the laser lab of the University of Twente. 


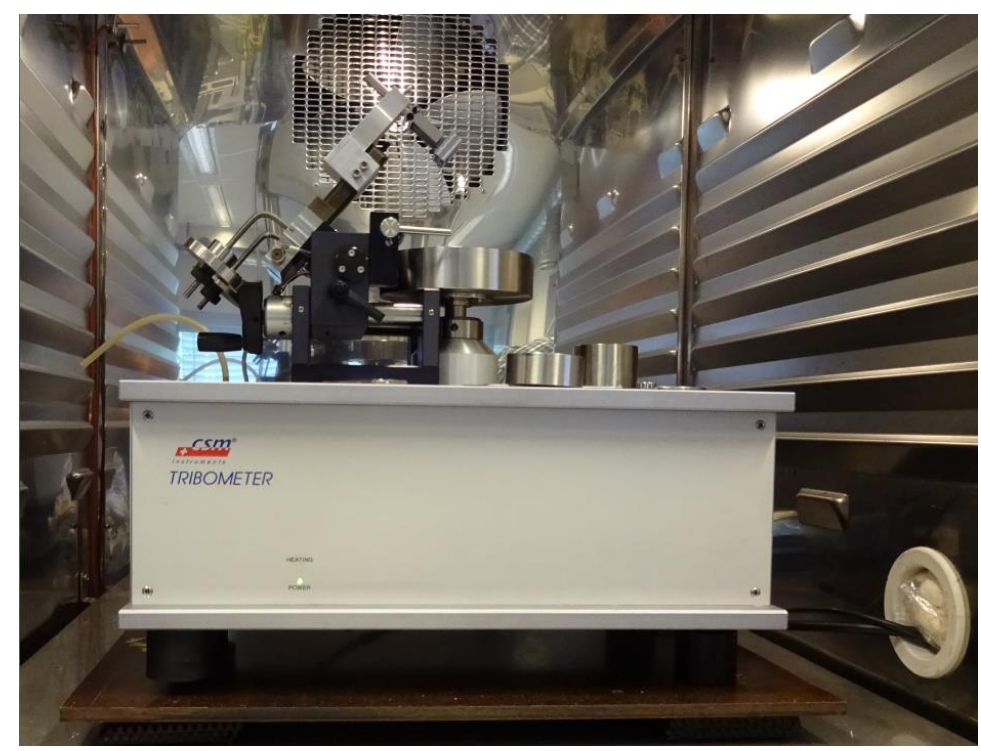

(a)

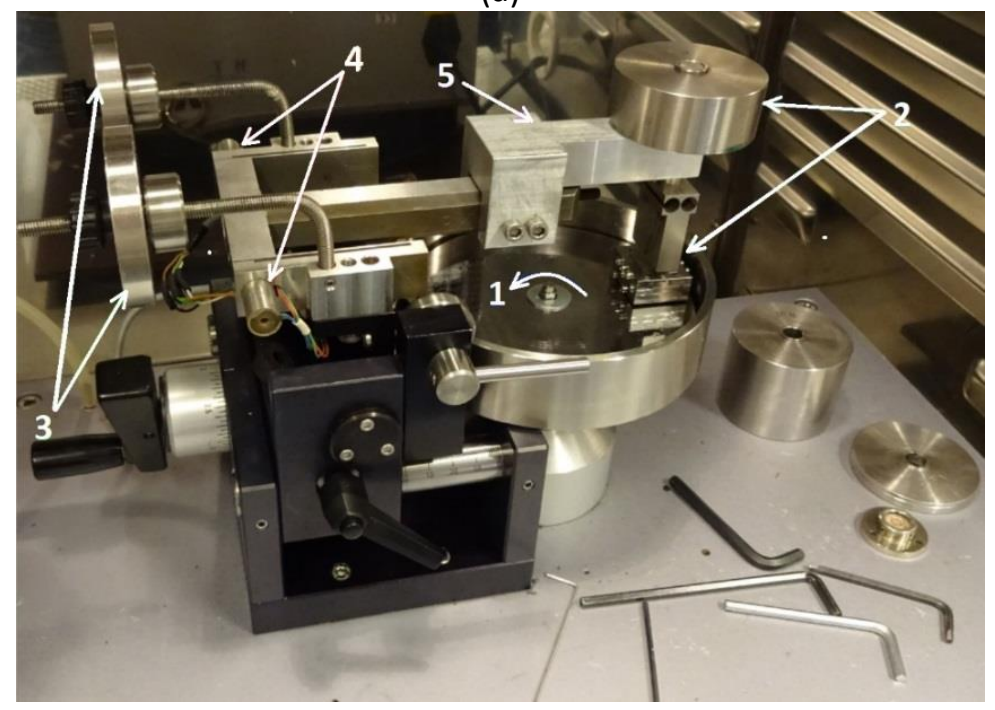

(b)

Figure 4.1. (a) pin-on-disk setup, (b) detail of the setup.

The setup is most frequently used to test the friction in a ball-on-disk configuration (point contact). Since we are interested in measuring friction in a flat-on-flat configuration, the setup has been modified to include a special holder to compensate for alignment issues. Further, a larger disk is used to make the contact inlet lubricant flow as uniform as possible (using the available setup).

Fig. 4.1.b shows the setup in more detail. The numbered parts are:

1. Smooth, stainless steel rotating disk

2. Sample holder with a dead weight on top

3. Counter weights to balance the mass of the arm (5), sample and sample holder (2)

4. Friction sensors

5. Arm extension 
The arm is mounted to the frame via flexures near the sensors. As the disk rotates the friction force will cause the arm to deflect. This deflection is measured and converted into a friction force. The coefficient of friction is obtained by dividing the friction force by the normal load (for the load caused by the weight) by software provided by CSEM Instruments.

\subsubsection{Sample holder}

To have an aligned flat-flat contact it is necessary to have a self-adjustment mechanism for the samples. By pivoting in two directions (parallel and perpendicular to the sliding direction) around the centre point of contact, it is possible for the sample to align. Therefore, by mounting such a sample holder into the pinon-disk machine the sample can self-adjust in parallel and perpendicular direction to the sliding direction. The schematic detail of the specimen holder is presented in Fig. 4.2. As can be seen, the specimen holder has three main parts. From top to bottom of the holder, the first part (1) the hinge, the second part (2) the elastic plates and the third part (3) the specimen grip (holder). The responsibility of the hinge is to adjust the sample with respect to misalignments, and irregularities on the disk in perpendicular direction (1) and moving direction (2). Moreover, by employing two thin plates as shown in Fig. 4.2 it is possible to have self-adjustment in moving direction. By employing this design, it is possible to eliminate the effect of misalignments in parallel sliding contact.

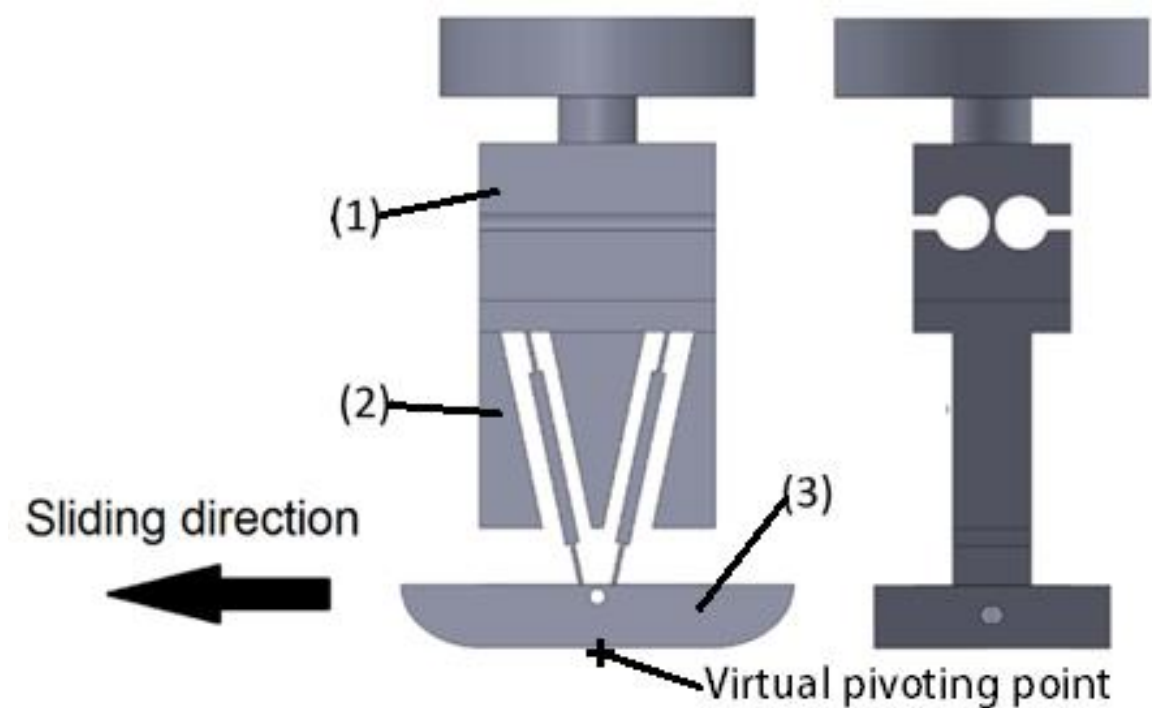

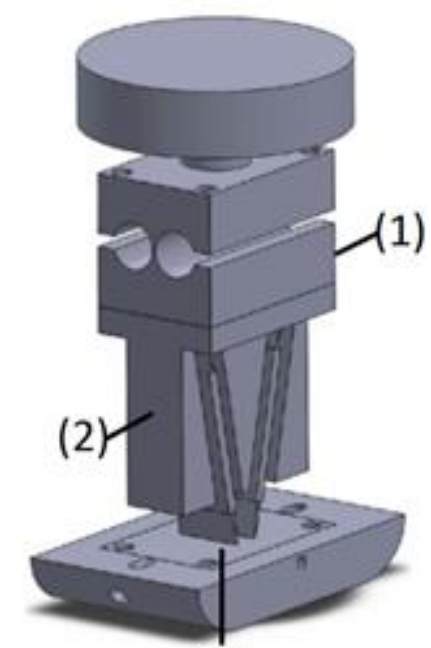

(3)

Figure 4.2. Schematic illustration of the designed specimen holder.

In Fig. 4.3, details of the designed specimen holder are shown, as well as the mounted specimen holder in the pin-on-disk tribometer. 


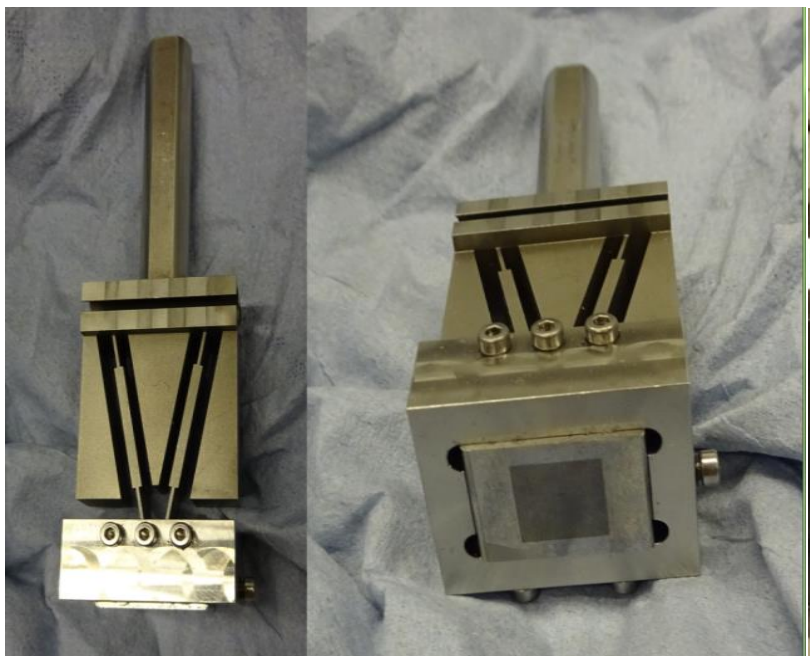

(a)

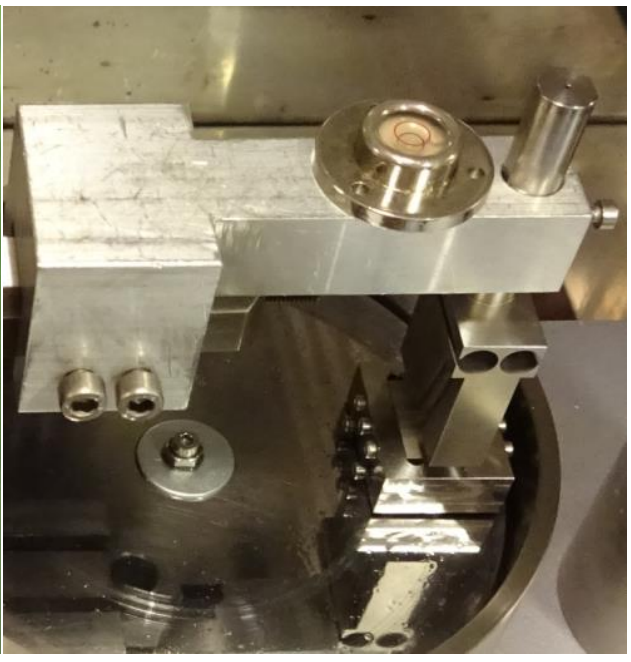

(b)

4.3. a) details of the sample holder, b) the mounted specimen holder.

\subsubsection{Samples}

As an example, the topography of a chevron cell measured with the confocal microscope (sub-section 4.2.4) is presented in Fig. 4.4.
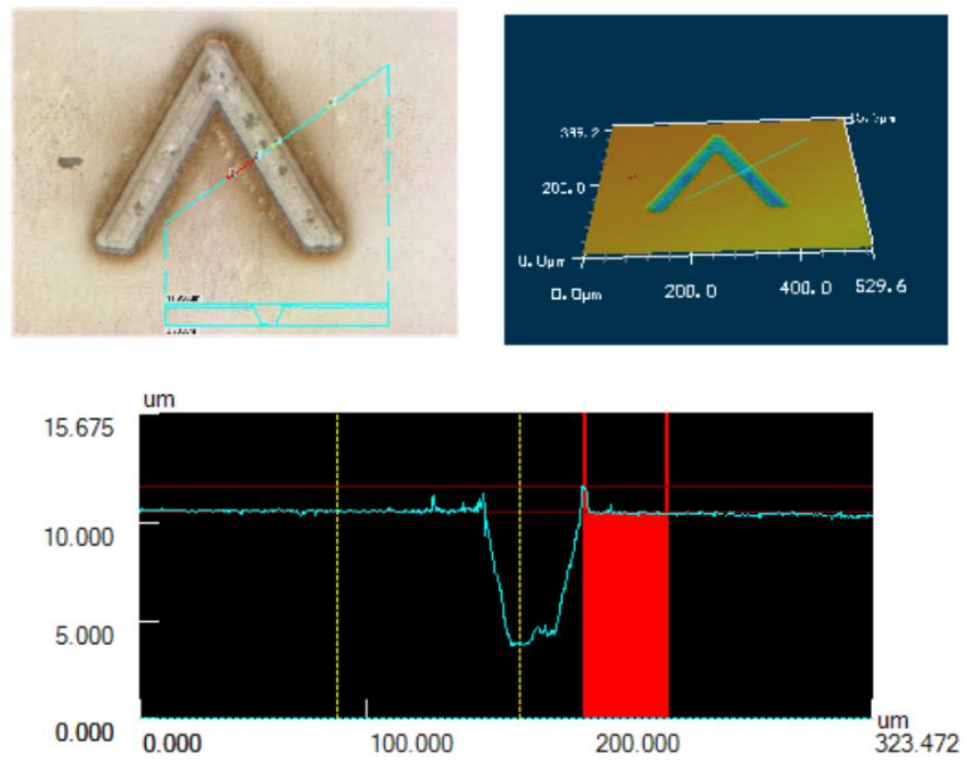

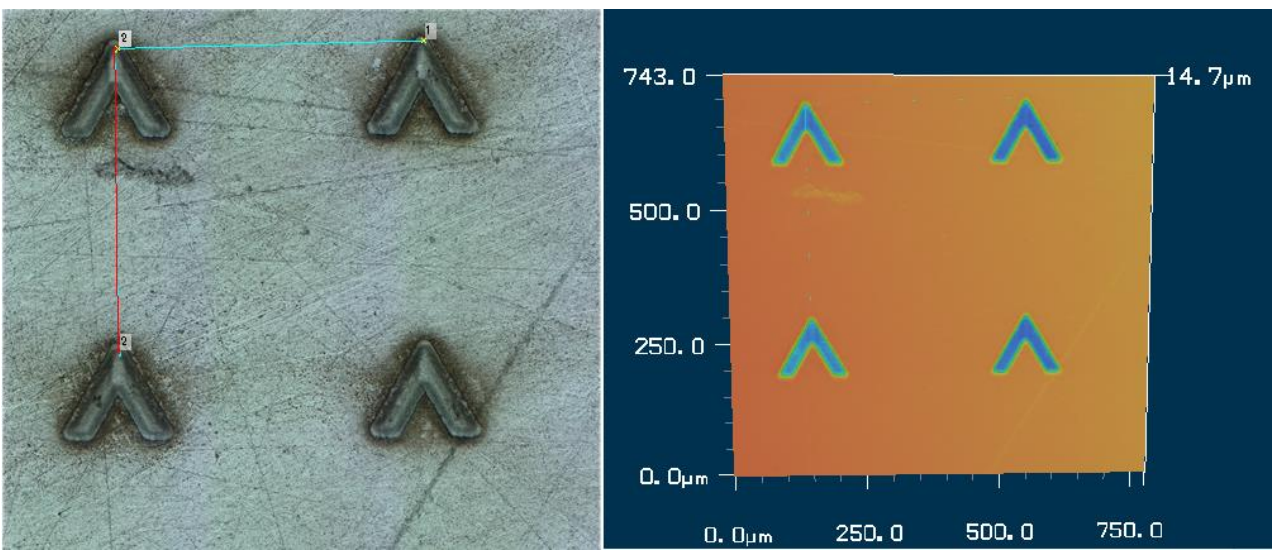

Figure 4.4. LST chevron pattern.

A schematic illustration of textured area of the samples is shown in Fig 4.5.

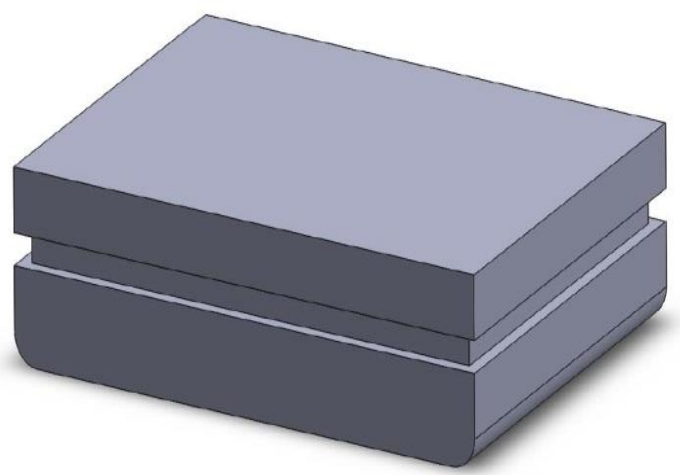

(a)

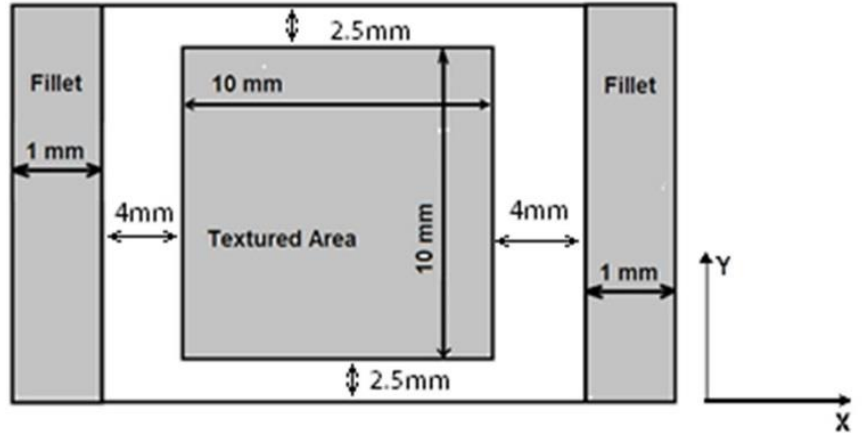

(b)

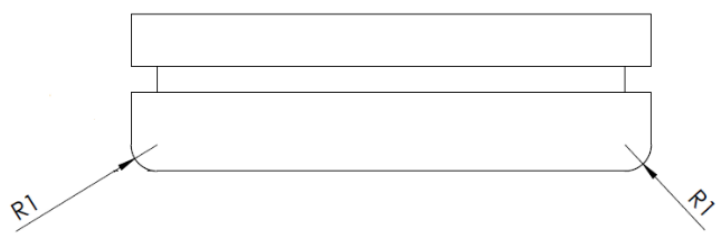

(c)

Figure 4.5. (a) 3D Schematic illustration of sample, (b) schematic illustration (bottom) and dimensions of the textured area on the samples, (c) geometry inlet and outlet of the specimen.

To stream a uniform lubricant film into the flat on flat contact area, the inlet and outlet fillets with radius of $1 \mathrm{~mm}$ are designed and the symmetrical design of samples and use of outlet fillet makes the outlet flow uniform as well. The textured area is located on the flat area of surface with $4 \mathrm{~mm}$ distance from the inlet and outlet fillets to avoid the possible influence of wedge effect caused by the curvature of fillets. Different depths, pitches and sizes of texturing are selected as presented in Table 4.2: 
Table 4.2. Textured samples characteristics.

\begin{tabular}{|c|c|c|c|c|c|c|c|}
\hline & Area of texture & Pitch & $\begin{array}{l}\text { depth } \\
(\mu m)\end{array}$ & $\begin{array}{l}\text { Cavity length } \\
\text { in X, } S(\mu \mathrm{m})\end{array}$ & $\begin{array}{l}\text { Cavity length } \\
\text { in } Y(\mu \mathrm{m})\end{array}$ & $\begin{array}{l}\text { Number of } \\
\text { arrays in } X\end{array}$ & $\begin{array}{l}\text { Number of } \\
\text { arrays in } Y\end{array}$ \\
\hline \multirow{5}{*}{ Grooves } & \multirow{5}{*}{$10 \mathrm{~mm} \times 10 \mathrm{~mm}$} & \multirow{4}{*}{0.4} & 5 & 100 & 10,000 & 40 & 1 \\
\hline & & & \multirow{3}{*}{10} & 50 & 10,000 & 80 & 1 \\
\hline & & & & 100 & 10,000 & 40 & 1 \\
\hline & & & & 200 & 10,000 & 20 & 1 \\
\hline & & 0.45 & 10 & 200 & 10,000 & 23 & 1 \\
\hline Chevrons & $10 \mathrm{~mm} \times 10 \mathrm{~mm}$ & 0.4 & 10 & 100 & 100 & 40 & 40 \\
\hline
\end{tabular}

\subsubsection{Confocal Microscope}

Surface topography analyses have been performed using a Keyence Colour 3D LASER Scanning Microscope. The control unit is a Keyence VK-9700K combined with a Keyence VK-9710K measurement unit, shown in Fig. 4.6.

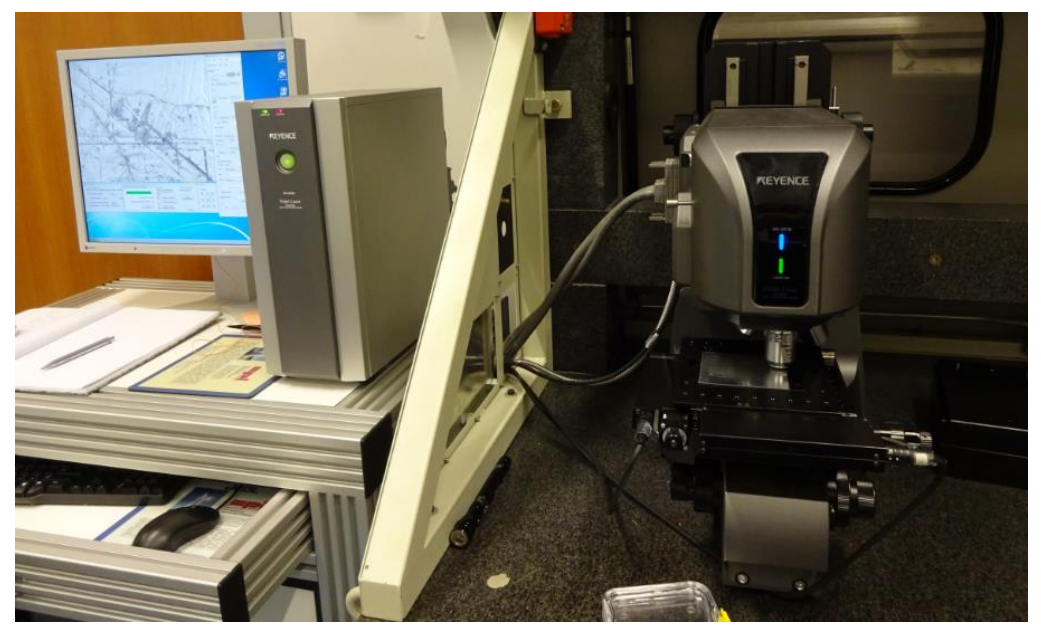

Figure 4.6. Keyence VK-9700K confocal microscope with a VK-9710K measurement unit.

\subsection{Results and discussion}

In this section the results of several friction experiments that were performed are presented.

\subsubsection{Non-textured surface}

First, initial measurements using a non-textured sample were performed. Based on the theory presented in chapter 2, in the case of non-textured samples no pressure is built up and therefore there is no lubricant film formation. Further, based on the study of the coefficient of friction and the effect of surface texturing on it, when there is no film formation the coefficient of friction is expected to be equal to the coefficient of friction under conditions of boundary lubrication.

If a misalignment exists, it is possible for the contact to operate in the boundary lubrication regime in one direction, but in the opposite direction (because of the existence of a wedge effect) the lubrication will be in a different lubrication regime and the coefficient of friction will be lower. For these reasons, friction is measured for several velocities; to check the reliability of specimen holder these measurements were also performed in the opposite sliding direction.

In Fig. 4.7 the Stribeck curve for the non-textured sample in both sliding directions is presented. The measurements were performed for four different velocities, and at least three measurements were taken 
for every value of a velocity. The error bars in these results show the maximum and minimum values measured and the line through the error bars shows the average measured value of the coefficient of friction as a function of velocity.

In Fig. 4.7 it is shown that for the non-textured samples the coefficient of friction is constant, i.e. no hydrodynamic action is found. The level for the coefficient of friction is in the range of $0.1-0.115$, and the mean value is approximately 0.11 .

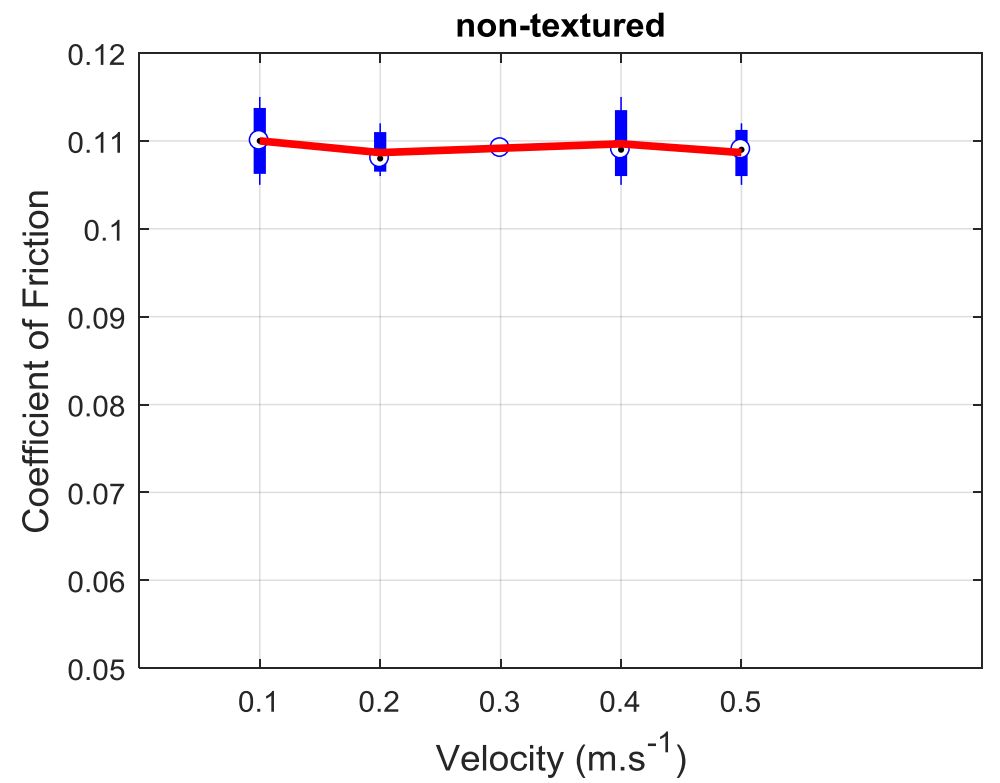

Figure 4.7. Coefficient of friction as a function of velocity for non-textured flat-flat contact $\left(F_{T}=5 \mathrm{~N}\right.$ and $\eta=32 \mathrm{mPa} . \mathrm{s}$ ).

\subsubsection{Measured results with textured surfaces}

After the validation of the sample holder's reliability, friction measurements using samples with different texturing shapes and dimensions were performed. Several measurements for grooved samples (Fig. 4.8) and one chevron sample (Fig. 4.9) were performed; the data extracted from these measurements is used to examine the numerical predictions with respect to the influence of texturing parameters, see section 4.3.3 et seq. The influence of cavity depth, size and pitch on friction was the focus of these measurements. 


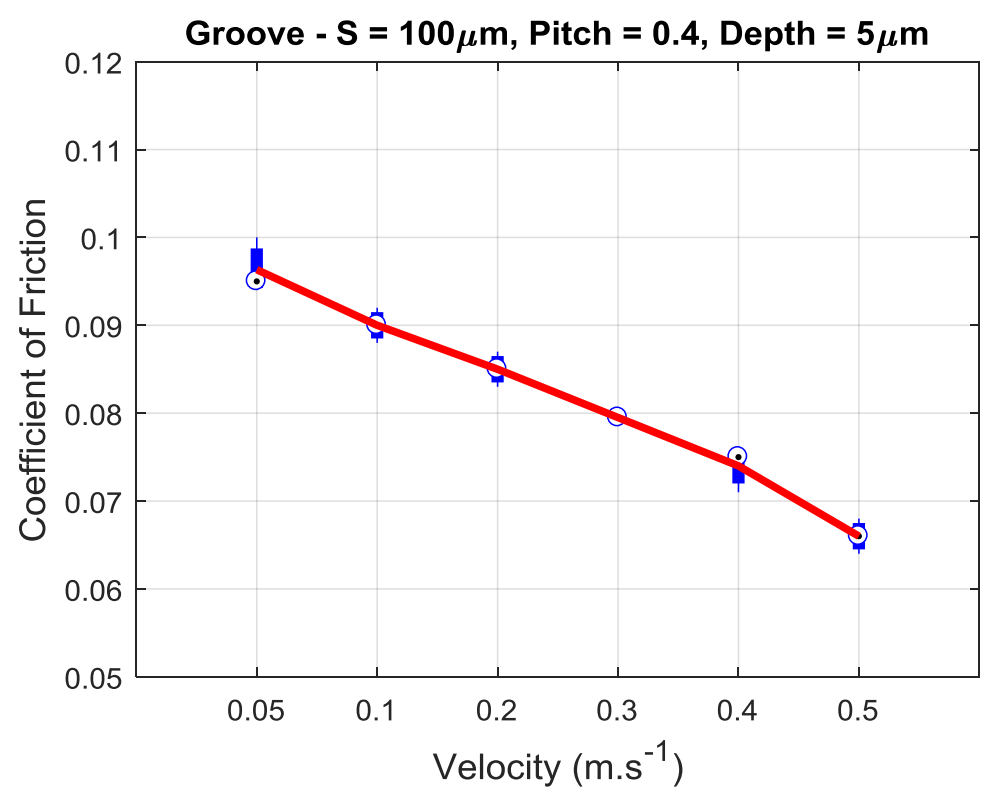

(a)

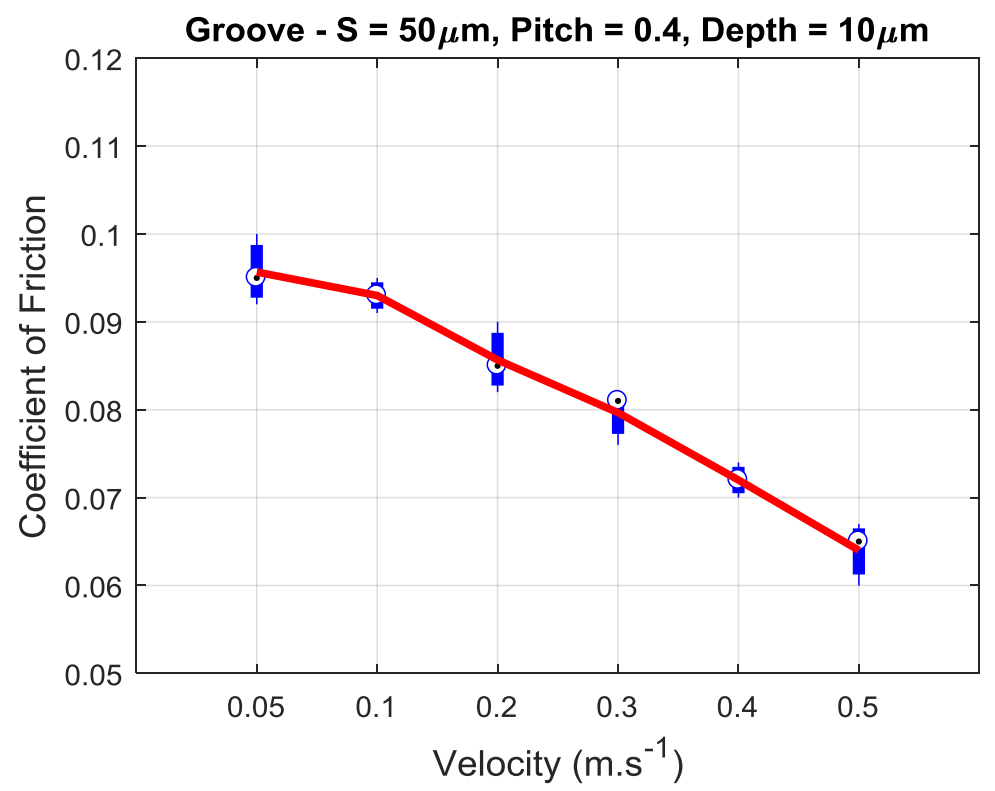

(b) 


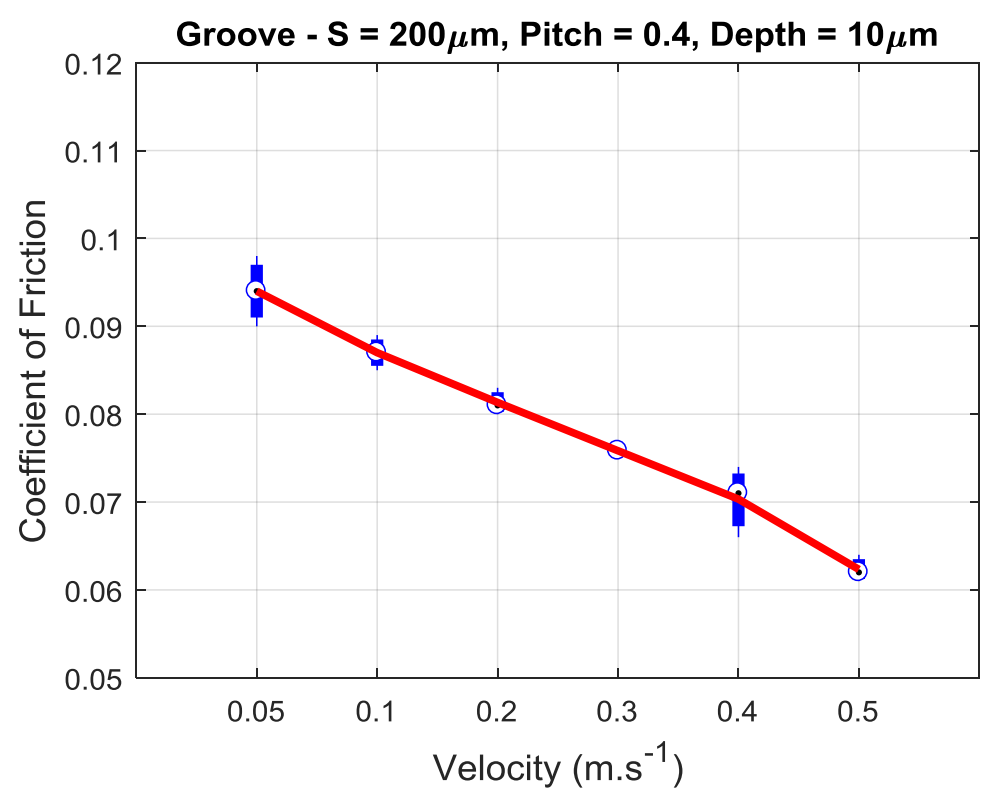

(c)

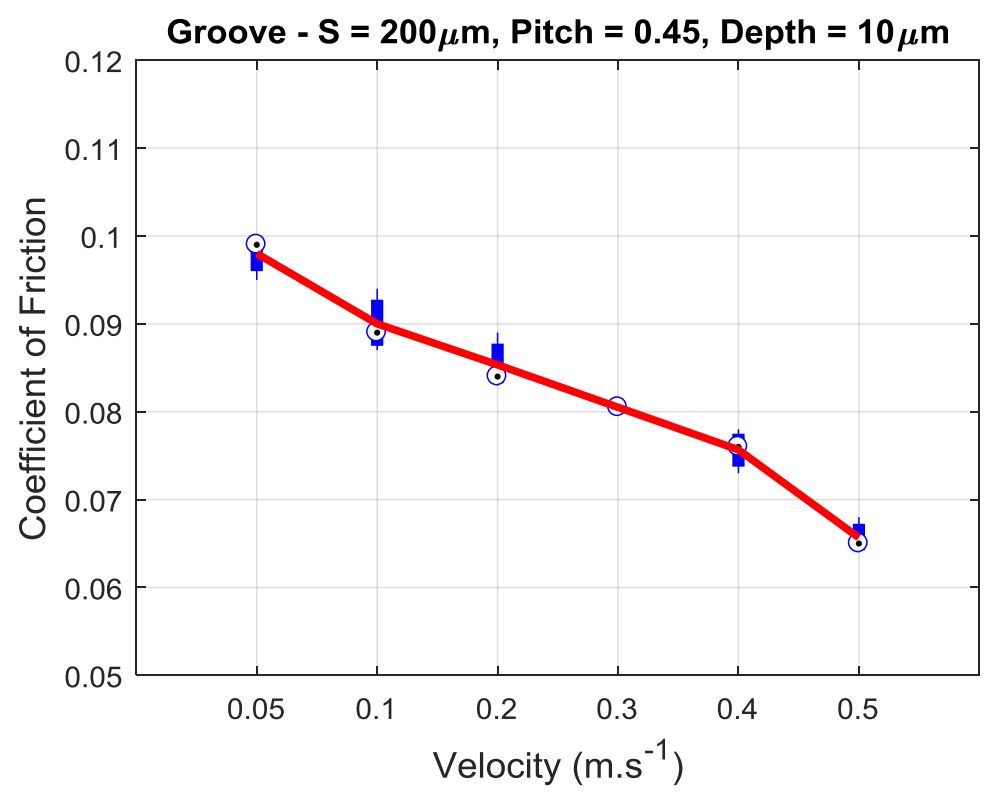

(d) 


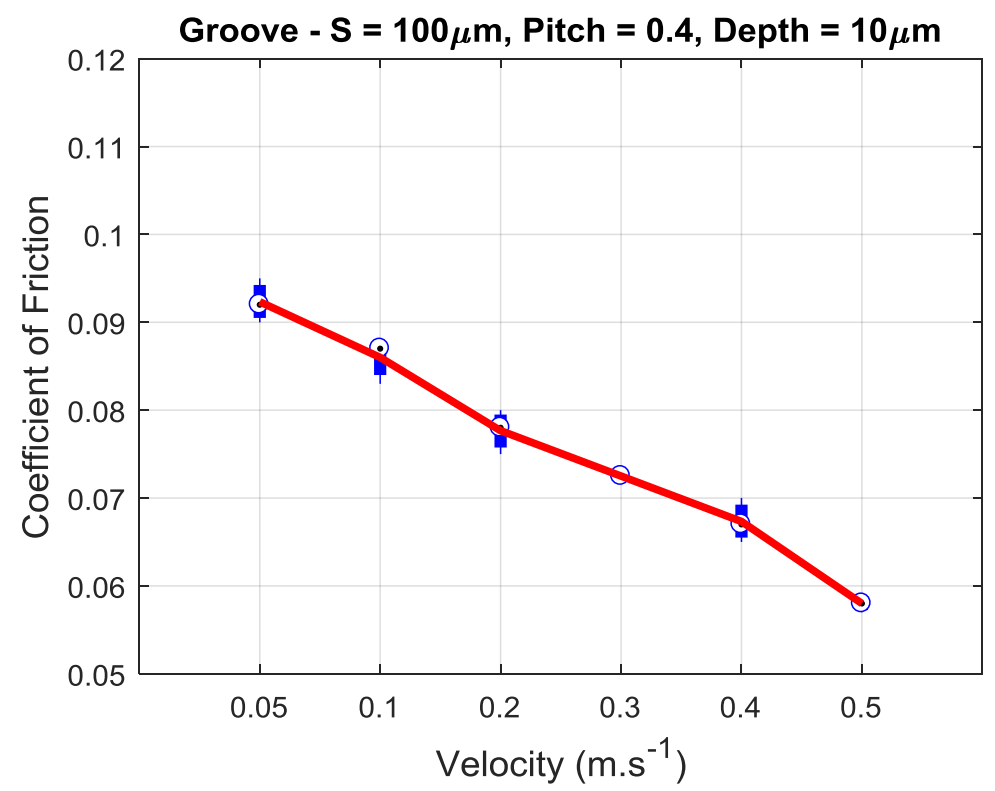

(e)

Figure 4.8. Coefficient of friction measurement results for grooved textured samples $\left(F_{T}=10 \mathrm{~N}\right.$ and $\eta=$ $32 m P a . s)$.

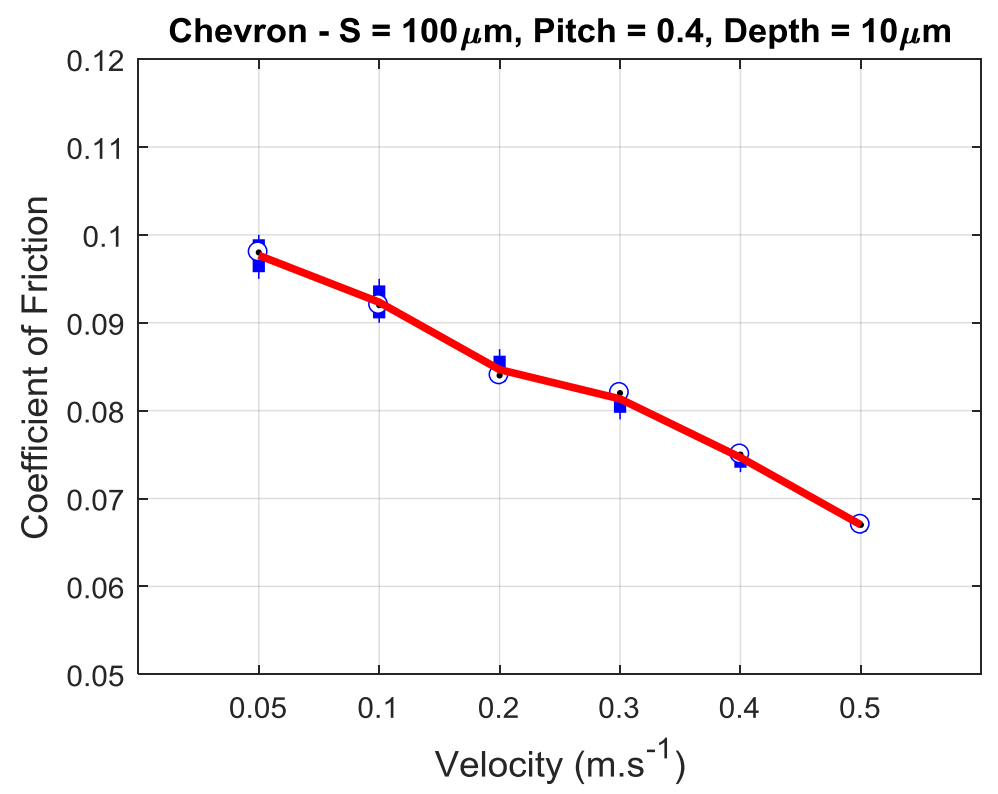

Figure 4.9. Coefficient of friction measurement results for chevron textured sample $\left(F_{T}=10 \mathrm{~N}\right.$ and $\eta=$ $32 m P a . s)$.

The figures show that the coefficient of friction in the boundary lubrication regime is around 0.10 , therefore in the subsections 4.3.3 to 4.3.6 where the calculated coefficient of friction and measured coefficient of friction are compared, this value for the $B L$ coefficient of friction is used as input for the friction model. 


\subsubsection{Pattern}

In this sub-section, the calculated coefficient of friction based on the numerical model presented in Chapter 3 is compared with the coefficient of friction measured with the pin-on-disk experiments. In Fig. 4.10 the numerical and experimental results of the coefficient of friction in the case of groove and chevron patterns are plotted. In this case the grooves have $10 \mu \mathrm{m}$ depth $\left(T_{d}\right), 100 \mu \mathrm{m}$ size $(S)$ and the pitch $\left(P_{x}\right)$ is equal to 0.4. The size of the chevrons used in this experiment is equal to the aforementioned values, i.e. $S=100 \mu m, T_{d}=10 \mu m$ and $P_{x}=0.4$.

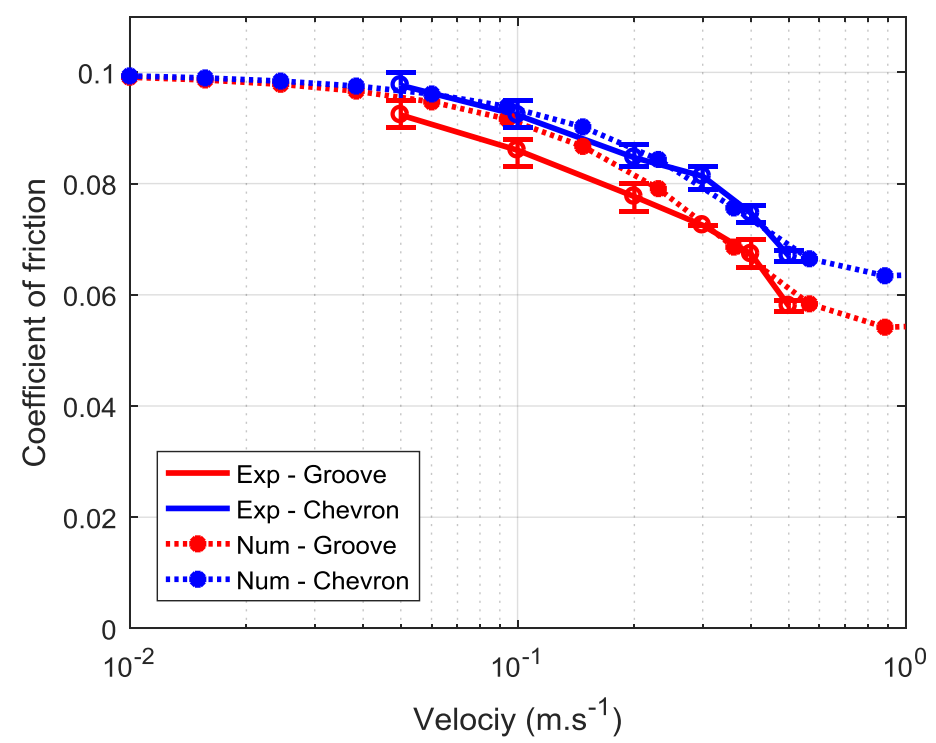

Figure 4.10. Numerical results vs experimental measurement results for groove and chevron patterns.

From Fig 4.10 it can be concluded that both the numerical and the experimental coefficient of friction show the same trend for the coefficient of friction as a function of velocity (Stribeck curve). The calculated coefficient of friction is in close agreement with the measured coefficient of friction and, finally, both the numerical and the experimental results show that the grooves are more effective than the chevrons for reducing the coefficient of friction. In both cases, the model is overestimating the measured coefficient of friction.

\subsubsection{Depth $\left(\boldsymbol{T}_{d}\right)$}

After checking the efficiency of the texture pattern, the influence of cavity depth on the frictional behaviour of the contacts was investigated. To achieve this goal, the coefficient of friction for two samples with a groove pattern is measured. The cavity depth for these two samples is respectively $5 \mu \mathrm{m}$ and $10 \mu \mathrm{m}$, the size parameter $(S)$ for both samples is equal to $100 \mu \mathrm{m}$ and the pitch parameter is equal to 0.4 . The measurements and calculations were based on the velocity range between $0.05 \mathrm{~m} . \mathrm{s}^{-1}$ and $0.5 \mathrm{~m} . \mathrm{s}^{-1}$. The numerical results and the experimental results are presented in Fig. 4.11.

The numerical results show in Fig. 4.11 that when the depth increases from $5 \mu \mathrm{m}$ to $10 \mu \mathrm{m}$, the coefficient of friction reduces and the trend in friction reduction is similar in both cases. A lower friction is achievable when the depth of the grooves is greater, due to a thicker film that is generated by applying grooves with a cavity depth close to the optimum value (see chapter 3 ). 


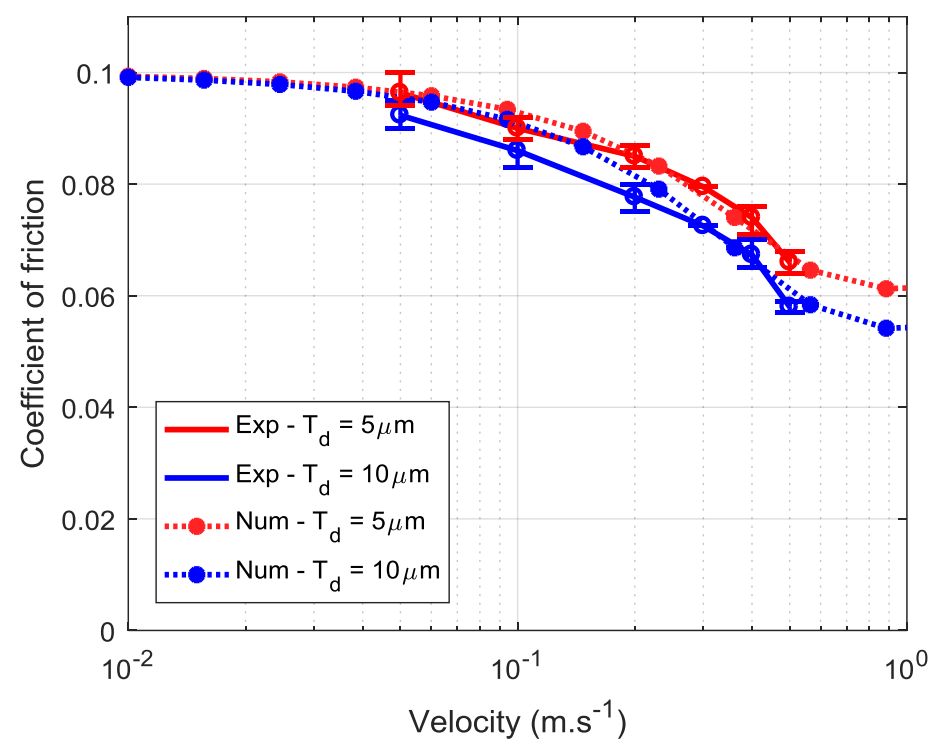

Figure 4.11. Comparison of numerical results vs experimental results for grooves with different cavity depth $\left(T_{d}\right)$.

\subsubsection{Size $(S)$}

These measurements were performed under $5 \mathrm{~N}$ normal load. The coefficient of friction for three samples with a groove pattern of a different size $(S)$ was measured. The cavity size for these three samples are $50 \mu \mathrm{m}, 100 \mu \mathrm{m}$ and $150 \mu \mathrm{m}$ respectively. The depth parameter $\left(T_{d}\right)$ for these samples is equal to $10 \mu \mathrm{m}$ and the pitch parameter $\left(P_{x}\right)$ is equal to 0.4 . The measurements and calculations were performed for a velocity range between $0.05 \mathrm{~m} / \mathrm{s}$ to $0.5 \mathrm{~m} / \mathrm{s}$. The numerical and experimental results are presented in Fig. 4.12.

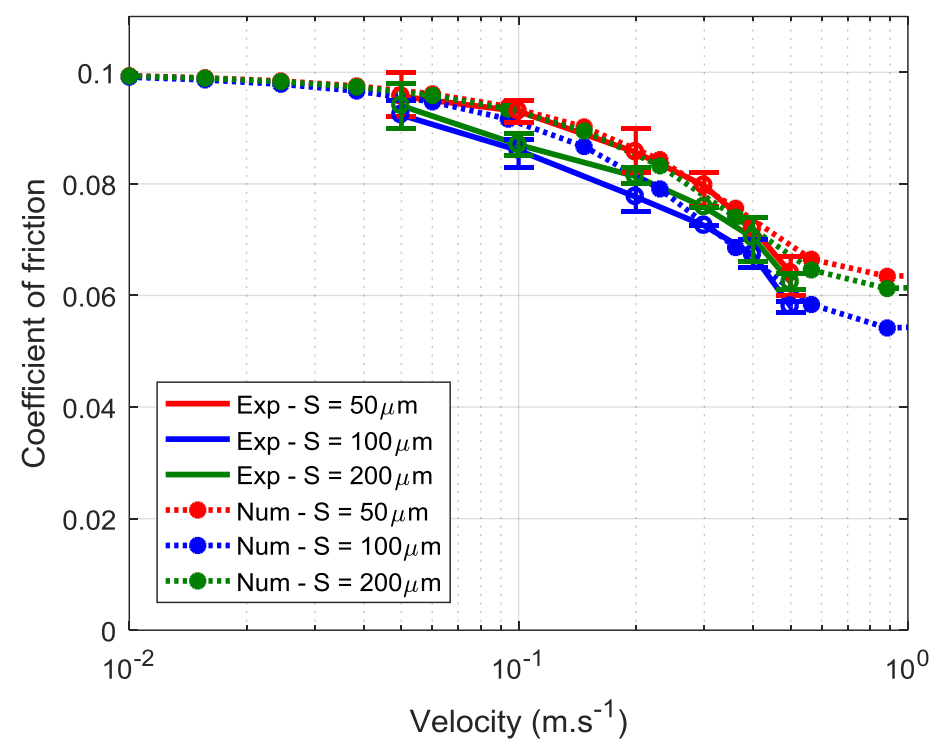

Figure 4.12. Comparison of numerical results vs experimental results for grooves with different cavity size (S). 
Fig. 4.12 shows that, like the previous numerical-experimental comparison, the trend of friction reduction is similar in both cases and they are in the same range. These results validate that, when size of grooves is around $100 \mu \mathrm{m}$ the lowest coefficient of friction is achievable, and the highest coefficient of friction for these three samples is obtained when the size of the cavities is around $50 \mu m$. This frictional behaviour of the groove pattern is due to the thicker film produced by applying grooves with the cavity size close to the optimum value.

\subsubsection{Pitch $\left(P_{x}\right)$}

The final parameter investigated is the pitch. The influence of this parameter on the frictional behaviour of sliding plane parallel contacts has been investigated experimentally and numerically. The coefficient of friction for two samples with a groove pattern is measured. The pitch parameters $\left(P_{x}\right)$ for these three samples are 0.40 and 0.45 , the depth parameter $\left(T_{d}\right)$ for these samples is equal to $10 \mu m$ and the size $(S)$ for both of them is equal to $100 \mu \mathrm{m}$. The numerical as well as the experimental results are presented in Fig. 4.13.

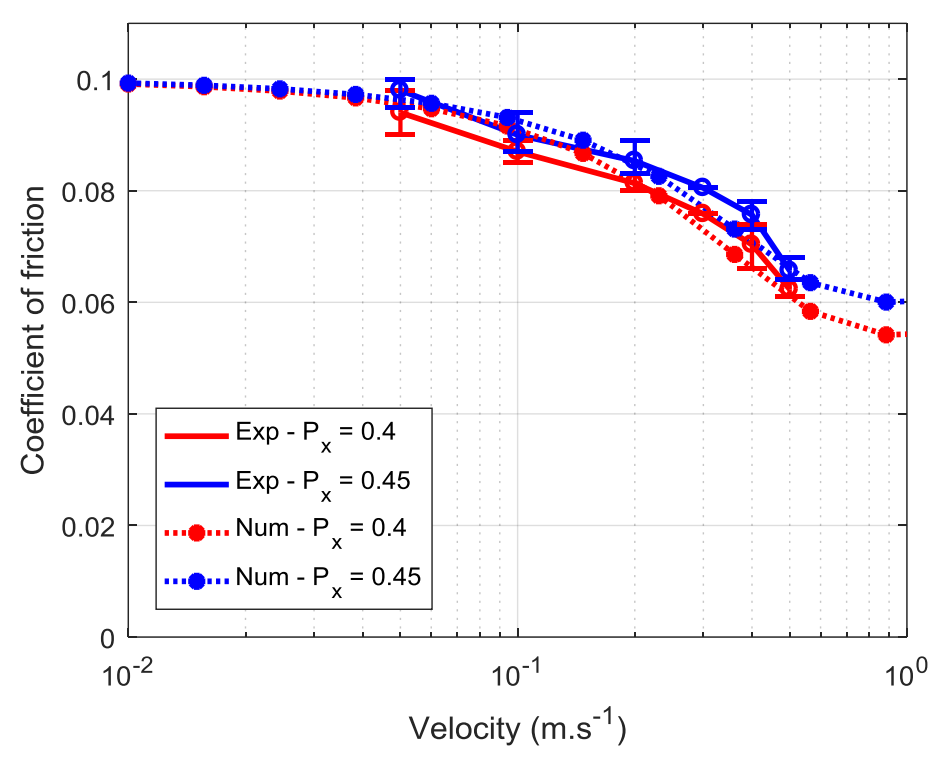

Figure 4.13. Comparison of numerical results vs experimental results for grooves with different pitch parameters $\left(P_{x}\right)$.

Fig. 4.13 shows that when $P_{x}$ is equal to 0.4 a lower coefficient of friction is obtained.

In section 4.3.1, the experimental results show that the grooves are more effective than the chevrons in reducing the coefficient of friction, which shows the dominant effect of more textured area than that created by applying chevrons. This effect was shown in the previous chapters (Chapter 2 and Chapter 3 ) too.

\subsection{Summary}

In this chapter, several experiments are performed in order to validate the mixed lubrication model with respect to texturing parameters. Firstly, measurements are performed to obtain the coefficient of friction under boundary lubrication condition by using a non-textured sample. This value is used as input in the mixed lubrication model. After that, measurements are performed to study the influence of different 
texturing parameters on friction and compared with simulations based on the model presented in this thesis. In conclusion:

- The trend of friction reduction is similar, both numerically and experimentally.

- The calculated coefficient of friction is in close agreement with the measured coefficient of friction.

- The grooves are more effective in reducing the coefficient of friction, both numerically and experimentally.

- The lowest friction is achievable when the depth of the grooves is around $10 \mu m$.

- These results validate that, when the size of grooves is around $100 \mu m$, the lowest coefficient of friction is achievable.

- In the case of a groove pattern the lowest coefficient of friction is achievable when $P_{x}=0.4$. 


\section{Chapter 5}

\section{Conclusions and Recommendations}

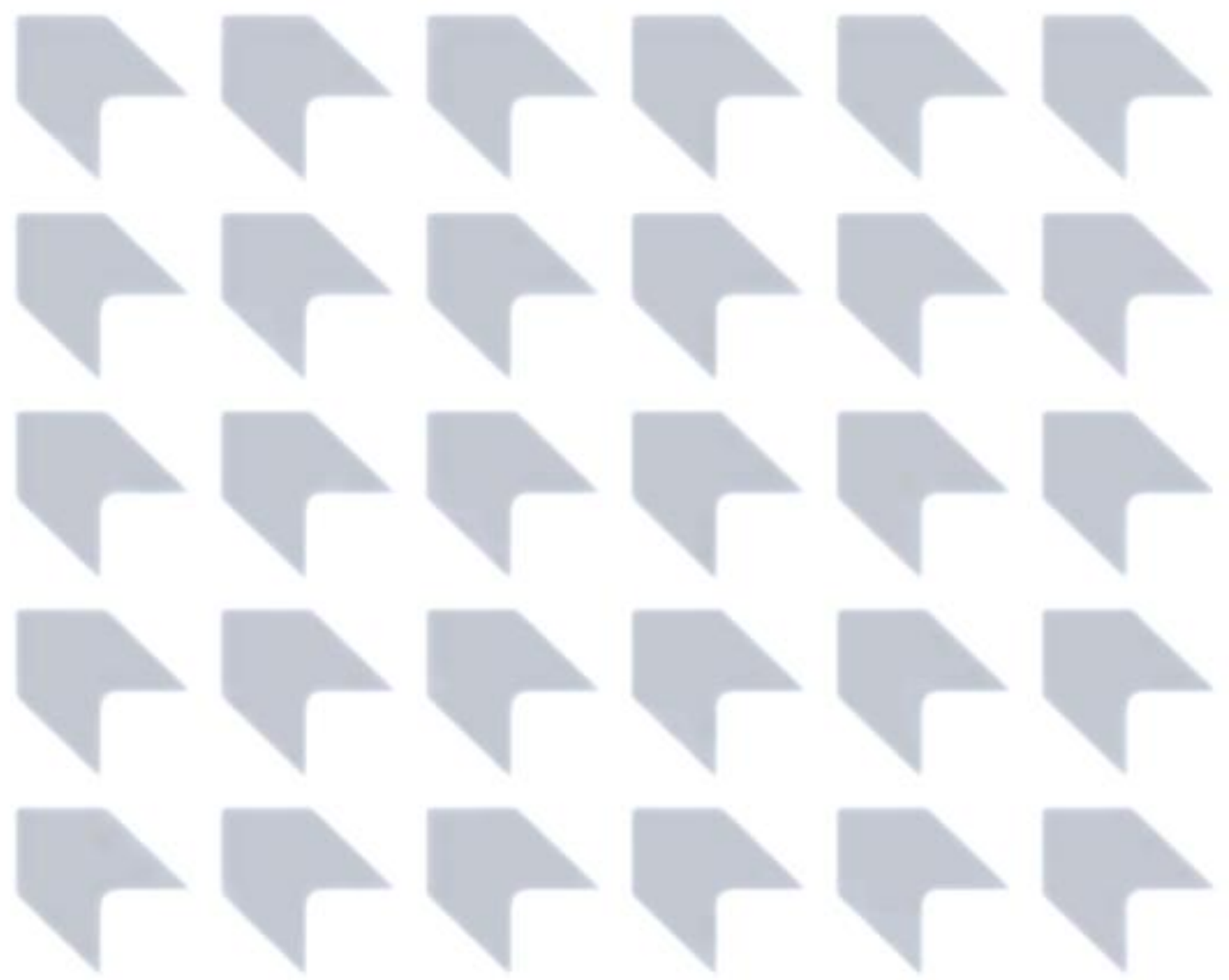




\subsection{Conclusions}

This chapter summarizes the conclusions of the previous chapters and provides recommendations for future research. In this thesis the following conclusions have been drawn:

\subsubsection{Effect of surface texturing on film thickness}

Simulations showed that the employment of surface texturing improves the lubricant film thickness of plane-parallel sliding contacts. These simulations are based on a modified Reynolds equation using the Elrod cavitation algorithm formulation and boundary slip conditions, solved by using the TDMA iterative algorithm.

The effect of several parameters, such as pattern type, cavity cross-sectional shape, depth and size on lubricant film thickness, has been studied. The effect of lubricant leakage for the groove pattern is investigated as well. Results showed that the groove pattern could form the thickest lubricant film due to higher textured area fraction.

The effect of the starvation on film formation of textured contacts is studied, along with the influence of texturing parameters on film thickness of starved lubricated contacts. This study showed that, for small values of the supplied lubricant thickness, the effect of starvation on the film thickness is greater. By increasing the lubricant supply $\left(h_{\text {oil }}\right)$, the texture geometry and dimensions gain a larger influence on the generated film thickness, while starvation reduces the effect of the texture on the film thickness.

In studying the influence of slip length on the film thickness and friction, it is clear that due to slip a thicker lubricant film is achievable, and with respect to the normalized coefficient of friction $\left(\mu^{*}\right)$ the increase in the slip length leads to a decrease in this parameter $\left(\mu^{*}\right)$.

\subsubsection{Surface texturing on frictional behaviour}

By combining the calculated film thickness with the deterministic asperity contact model, the coefficient of friction is calculated under mixed lubrication conditions. The effect of several parameters on the frictional behaviour of lubricated parallel siding contacts such as pattern type, depth, size and texture pitch has been studied. It is shown that when the distances between texture cells are the same, i.e. the pitch is constant, the groove pattern is more successful in generating load-carrying capacity, because of the larger textured area fraction per unit area. Chevrons are more beneficial than the triangular pockets. From a parametric study of different patterns the following conclusions have been drawn:

- In the case of a groove pattern, the optimum cavity size is approximately $S=100 \mu \mathrm{m}$ and in the case of chevrons, the optimum cavity size is approximately $S=150 \mu \mathrm{m}$.

- The optimum depth for the different patterns is in the range of $10 \mu \mathrm{m}$.

Further, the influence of starvation on the frictional behaviour of textured surfaces was studied. In the case of starved lubrication, when the value on input film thickness $\left(h_{\text {oil }}\right)$ decreases the starvation effect gains a larger effect on the film thickness. Therefore, the effect of variation of the texture parameters (Pitch, Depth and Size) on the coefficient of friction is less sensitive than for non-starved lubricated contacts.

\subsubsection{Experimental validation of the friction model}

In order to validate the mixed lubrication friction model with respect to texturing parameters, several experiments were performed. Firstly, measurements were performed to obtain the coefficient of friction under boundary lubrication condition by using a non-textured sample. After that, measurements were taken to study the influence of different texturing parameters on friction. The results are then compared 
with simulations based on the friction model presented in this thesis. These measurements showed that the trend of friction reduction, both numerically and experimentally, is similar in both cases and the calculated coefficient of friction is in close agreement with the measured coefficient of friction.

Furthermore, measurements proved that the grooves are more effective in reducing the coefficient of friction both numerically and experimentally and the lowest friction is obtained when the depth of the grooves is around $10 \mu \mathrm{m}$. These results validate that the lowest coefficient of friction is achievable when the size of grooves is around $100 \mu m$ and $P_{x}=0.4$.

\subsection{Remarks}

In this study, surface texturing showed a promising effect on friction reduction in plane parallel sliding lubricated contacts. By optimizing the geometrical parameters of the texture patterns it is possible to improve this effect. Boundary slip as another surface modification method could be helpful to boost the film thickness in plane parallel sliding lubricated contacts; and the effect of combining these two methods on the film thickness with an optimized texture geometry can maximize the lubricant film thickness.

As it is shown in Chapter 3, by employing surface texturing it is possible to shift the transition between the mixed lubrication and hydrodynamic lubrication regimes. Also, the coefficient of friction at lower velocities can be reduced. This effect is also measured and presented in Chapter 4 of this thesis. Although the existence of starvation in textured lubricated contacts can limit the influence of surface texturing, the film thickness and reduce friction can nevertheless be improved by surface texturing.

\subsection{Recommendations for future research}

- Although this study focuses mainly on texturing patterns, the algorithm and software developed in this study is capable of calculating the friction of more complex patterns.

- In industrial applications the effect of temperature on lubricant properties, in particular the velocity, could be worthwhile, therefore to improve the model it is suggested to add a thermal model.

- Due to a relatively low normal load in this study, elastic bulk deformation was not investigated. The addition of elastic bulk deformation could be useful to expand the application of this model.

- In this work the influence of boundary slip and its combination with surface texturing is studied. A further study on this topic and with complex texturing patterns is suggested. 
Appendix A

Determination of Roughness Parameters

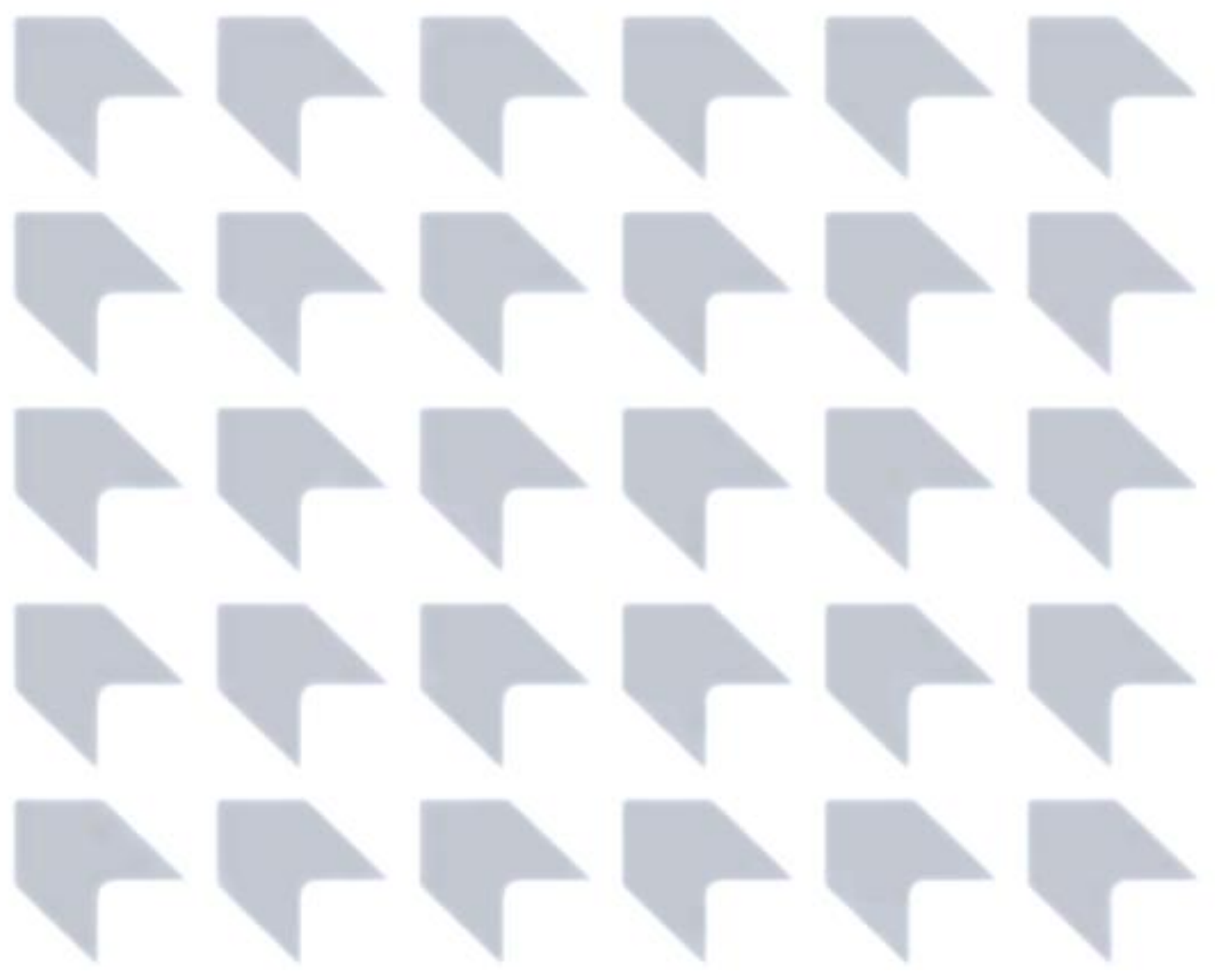


In this study, calculations were performed using the roughness measured by images extracted from the laser microscope; these images are obtained from roughness height measurements. In this appendix, one of these images is shown as an example and the equations for calculating the roughness parameters are presented as well.

\section{A.1. Roughness Measurement}

In order to calculate the boundary lubrication component, the roughness measurement is essential. As mentioned in Chapter 3, in the case of the deterministic approach, the real measured height of asperities is needed to calculate the separation between the opposing surfaces. In order to obtain the height of the asperities, the surface topography for textured surfaces is measured. These analyses have been performed using a Keyence Color 3D Scanning Microscope, which uses a violet laser with $\lambda=388 \mathrm{~nm}$. The result from a roughness measurement sample is presented in Figure A1 (standard lens 50x).

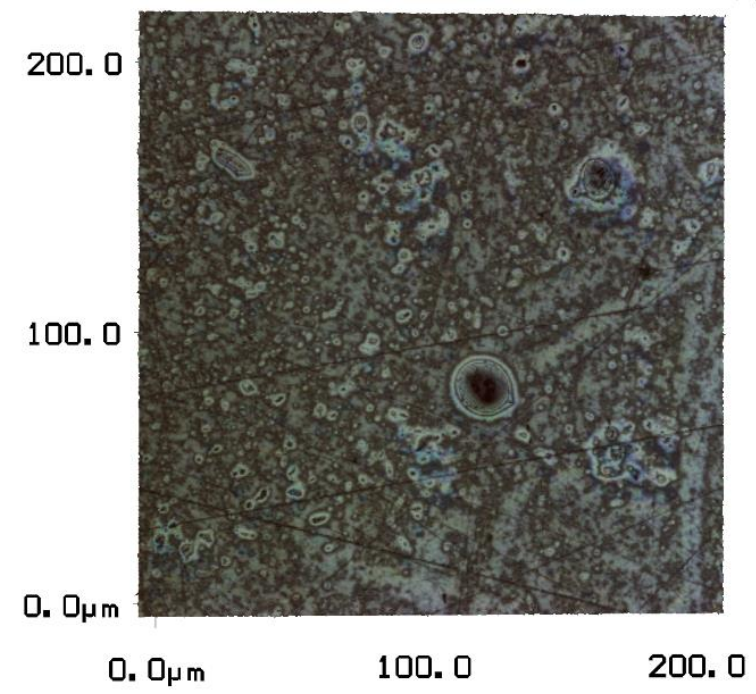

Figure A1. Laser scanning microscope image of a surface (non-textured area of sample).

By using the measured data for the surface roughness, the 3D illustration is calculated (see Figure A2).

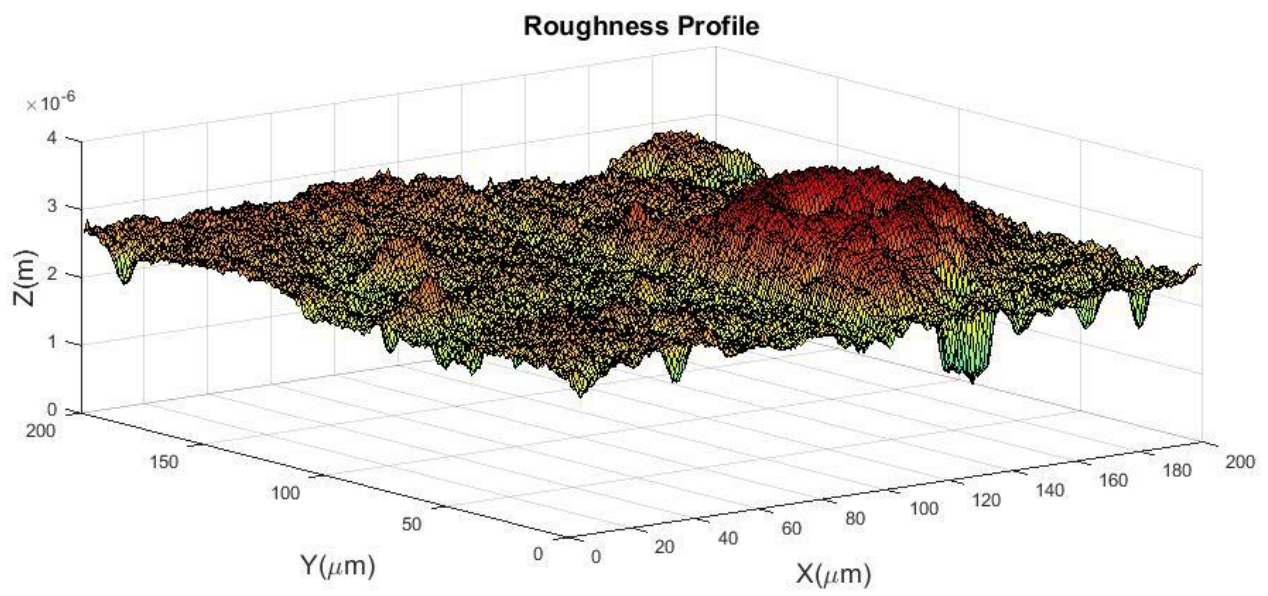

Figure A2. Roughness measurement surface based on the measured roughness by Laser Scanning Microscope measurements, in this case $R_{a}=0.11 \mu \mathrm{m}$. 
To compare the measured roughness with the Gaussian roughness distribution, the probability density of asperities against the dimensionless asperity height is shown in Figure A3. In this figure the Gaussian probability density distribution is shown with the red line and blue bars present the real measured probability density distribution.

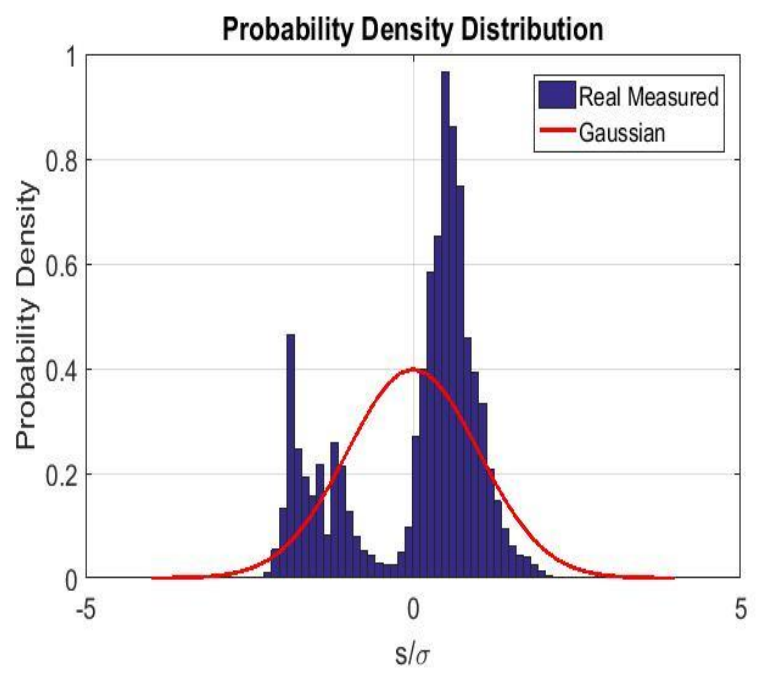

Figure A3. Distribution of surface heights as a function of the dimensionless asperity height $(s / \sigma)$.

The definition of a summit in this work is that it is higher than its eight neighbouring points (see Figure A4).

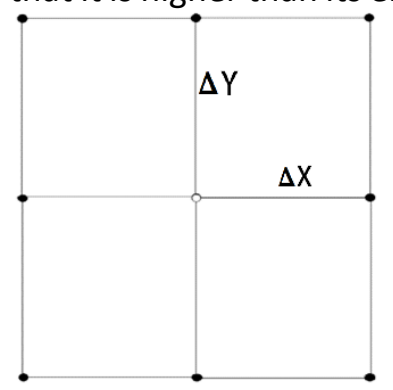

Figure A4. Definition of a summit.

The radius of an asperity is calculated using the three-point definition:

$$
\begin{aligned}
& \beta_{x}^{-1}=\frac{z_{x-\Delta x, y}-2 z_{x, y}+z_{x+\Delta x, y}}{\Delta x^{2}} \\
& \beta_{y}^{-1}=\frac{z_{x-\Delta x, y}-2 z_{x, y}+z_{x+\Delta x, y}}{\Delta y^{2}}
\end{aligned}
$$

where $\beta_{x}$ and $\beta_{y}$ are the radii in, respectively, the $x$ and the $y$ directions, $z_{x, y}$ is the local surface height at location $(x, y)$ and $\Delta x, \Delta y$ is the pixel size. The combined summit radius $\beta_{i}$ of the radii in the two perpendicular directions $\beta_{x}$ and $\beta_{y}$ is obtained by:

$$
\beta_{i}=\sqrt{\beta_{x i} \cdot \beta_{y i}}
$$

As shown in Figure 3.3 and Figure 3.9 the combined summit radius $\left(\beta_{i}\right)$ is an input of the mixed lubrication model. Moreover, the average summit radius $(\bar{\beta})$ is given by: 


$$
\bar{\beta}=\frac{1}{n} \sum_{i=1}^{n} \beta_{i}
$$

The average radius of asperity from Figure $A 2$ is equal to $4.8 \times 10^{-8} \mathrm{~m}$. 


\section{References}

[1] Galda, L., Pawlus, P., and Sep, J., 2009, "Dimples shape and distribution effect on characteristics of Stribeck curve," Tribology International, 42(10), pp. 1505-1512.

[2] Tian, H., Saka, N., and Suh, N., 1989, "Boundary Lubrication Studies on Undulated Titanium Surfaces," Tribology Transactions - TRIBOL TRANS, 32, pp. 289-296.

[3] Williams, J. A., and Hyncica, A. M., 1992, "Mechanisms of abrasive wear in lubricated contacts," Wear, 152(1), pp. 57-74.

[4] Volchok, A., Halperin, G., and Etsion, I., 2002, "The effect of surface regular microtopography on fretting fatigue life," Wear, 253(3-4), pp. 509-515.

[5] Steinhoff, K., Rasp, W., and Pawelski, O., 1996, "Development of deterministic-stochastic surface structures to improve the tribological conditions of sheet forming processes," Journal of Materials Processing Technology, 60(1-4), pp. 355-361.

[6] Kligerman, Y., and Etsion, I., 2001, "Analysis of the hydrodynamic effects in a surface textured circumferential gas seal," Tribology Transactions, 44(3), pp. 472-478.

[7] Etsion, I., Kligerman, Y., and Halperin, G., 1999, "Analytical and Experimental Investigation of LaserTextured Mechanical Seal Faces," Tribology Transactions, 42(3), pp. 511-516.

[8] Raeymaekers, B., Etsion, I., and Talke, F. E., 2007, "Enhancing tribological performance of the magnetic tape/guide interface by laser surface texturing," Tribology Letters, 27(1), pp. 89-95.

[9] Etsion, I., Halperin, G., Brizmer, V., and Kligerman, Y., 2004, "Experimental Investigation of Laser Surface Textured Parallel Thrust Bearings," Tribology Letters, 17(2), pp. 295-300.

[10] Ryk, G., Kligerman, Y., and Etsion, I., 2002, "Experimental Investigation of Laser Surface Texturing for Reciprocating Automotive Components," Tribology Transactions, 45(4), pp. 444-449.

[11] Yu, H., Wang, X., and Zhou, F., 2010, "Geometric Shape Effects of Surface Texture on the Generation of Hydrodynamic Pressure Between Conformal Contacting Surfaces," Tribology Letters, 37(2), pp. 123-130.

[12] Costa, H. L., and Hutchings, I. M., 2007, "Hydrodynamic lubrication of textured steel surfaces under reciprocating sliding conditions," Tribology International, 40(8), pp. 1227-1238.

[13] Siripuram, R. B., and Stephens, L. S., 2004, "Effect of deterministic asperity geometry on hydrodynamic lubrication," Journal of Tribology, 126(3), pp. 527-534.

[14] Ma, C., and Zhu, H., 2011, "An optimum design model for textured surface with elliptical-shape dimples under hydrodynamic lubrication," Tribology International, 44(9), pp. 987-995.

[15] Kovalchenko, A., Ajayi, O., Erdemir, A., Fenske, G., and Etsion, I., 2005, "The effect of laser surface texturing on transitions in lubrication regimes during unidirectional sliding contact," Tribology International, 38(3), pp. 219-225.

[16] Kovalchenko, A., Ajayi, O., Erdemir, A., Fenske, G., and Etsion, I., 2004, "The effect of laser texturing of steel surfaces and speed-load parameters on the transition of lubrication regime from boundary to hydrodynamic," Tribology Transactions, 47(2), pp. 299-307.

[17] Tauviqirrahman, M., Ismail, R., Jamari, and Schipper, D. J., 2013, "Optimization of the complex slip surface and its effect on the hydrodynamic performance of two-dimensional lubricated contacts," Computers \& Fluids, 79(0), pp. 27-43.

[18] Ma, G. J., Wu, C. W., and Zhou, P., 2007, "Wall slip and hydrodynamics of two-dimensional journal bearing," Tribology International, 40(7), pp. 1056-1066.

[19] Tauviqirrahman, M., 2013, "Lubricated MEMS: effect of boundary slippage and texturing, PhD Thesis," PhD PhD Thesis, Unversity of Twente, Enschede.

[20] Tauviqirrahman, M., Ismail, R., Jamari, J., and Schipper, D. J., 2013, "Combined effect of texturing and boundary slippage in lubricated sliding contacts," Tribology International, 66(0), pp. 274-281.

[21] Fogg, A., 1946, "Fluid film lubrication of parallel thrust surfaces," Proceedings of the Institution of Mechanical Engineers, 155(1), pp. 49-67. 
[22] Pinkus, O., and Sternlicht, B., 1961, Theory of Hydrodynamic Lubrication, University Microfilms.

[23] Spikes, H. A., 2003, "The half-wetted bearing. Part 1: Extended Reynolds equation," Proceedings of the Institution of Mechanical Engineers, Part J: Journal of Engineering Tribology, 217(1), pp. 1-14.

[24] Feldman, Y., Kligerman, Y., Etsion, I., and Haber, S., 2006, "The Validity of the Reynolds Equation in Modeling Hydrostatic Effects in Gas Lubricated Textured Parallel Surfaces," Journal of Tribology, 128(2), pp. 345-350.

[25] Payvar, P., and Salant, R. F., 1992, "Computational method for cavitation in a wavy mechanical seal," Journal of Tribology, 114(1), pp. 199-204.

[26] Wedeven, L. D., Evans, D., and Cameron, A., 1971, "Optical analysis of ball bearing starvation," J Lubric Technol Trans ASME, 93 Ser F(3), pp. 349-363.

[27] Kingsbury, E., 1973, "Cross flow in a starved EHD contact," ASLE Transactions, 16(4), pp. 276-280.

[28] Chiu, Y. P., 1974, "An analysis and prediction of lubricant film starvation in rolling contact systems," ASLE Transactions, 17(1), pp. 22-35.

[29] Elrod, H. G., and Adams, M. L., 1975, "A computer program for cavitation and starvation problems.."

[30] Chevalier, F., Lubrecht, A. A., Cann, P. M. E., Colin, F., and Dalmaz, G., 1995, "Starved film thickness: a qualitative explanation," Tribology Series, 30, pp. 249-257.

[31] Chevalier, F., Lubrecht, A. A., Cann, P. M. E., Colin, F., and Dalmaz, G., 1998, "Film thickness in starved EHL point contacts," Journal of Tribology, 120(1), pp. 126-132.

[32] Damiens, B., Venner, C. H., Cann, P. M. E., and Lubrecht, A. A., 2004, "Starved lubrication of elliptical EHD contacts," Journal of Tribology, 126(1), pp. 105-111.

[33] Faraon, I., and Schipper, D. J., 2007, "Stribeck curve for starved line contacts," Journal of Tribology, 129(1), pp. 181-187.

[34] Odyck, D. E. A. v., 2001, "Stokes flow in thin films,"PhD thesis, University of Twente, Enschede.

[35] Elrod, H. G., 1973, "Thin-Film Lubrication Theory for Newtonian Fluids With Surfaces Possessing Striated Roughness or Grooving," Journal of Lubrication Technology, 95(4), pp. 484-489.

[36] Jakobsson, B., and Floberg, L., 1957, The finite journal bearing, considering vaporization: (Das Gleitlager von endlicher Breite mit Verdampfung), Chalmers University of Technology, Chalmers Tekniska Högskolas handlingar ; Avd. Maskinteknik

[37] Olsson, K. O., 1965, Cavitation in Dynamically Loaded Bearings, Scandinavian University Books, Chalmers University of Technology, Institute of Machine Elements. Report.

[38] Coyne, J. C., and Elrod, H. G., 1965, Conditions for the Rupture of a Lubrication Film.

[39] Braun, M. J., and Hannon, W. M., 2010, "Cavitation formation and modelling for fluid film bearings: A review," Proceedings of the Institution of Mechanical Engineers, Part J: Journal of Engineering Tribology, 224(9), pp. 839-863.

[40] Xiong, S., and Wang, Q. J., 2012, "Steady-state hydrodynamic lubrication modeled with the payvarsalant mass conservation model," Journal of Tribology, 134(3).

[41] Dobrica, M. B., Fillon, M., Pascovici, M. D., and Cicone, T., 2010, "Optimizing surface texture for hydrodynamic lubricated contacts using a mass-conserving numerical approach," Proceedings of the Institution of Mechanical Engineers, Part J: Journal of Engineering Tribology, 224(8), pp. 737-750.

[42] Brizmer, V., Kligerman, Y., and Etsion, I., 2003, "A Laser Surface Textured Parallel Thrust Bearing," Tribology Transactions, 46(3), pp. 397-403.

[43] Guo, Z., Yuan, C., Liu, P., Peng, Z., and Yan, X., 2013, "Study on influence of cylinder liner surface texture on lubrication performance for cylinder liner-piston ring components," Tribol Lett, 51(1), pp. 9-23.

[44] Grabon, W., Pawlus, P., Wos, S., Koszela, W., and Wieczorowski, M., 2018, "Evolutions of cylinder liner surface texture and tribological performance of piston ring-liner assembly," Tribology International, 127, pp. 545-556. 
[45] Grabon, W., Pawlus, P., and Sep, J., 2010, "Tribological characteristics of one-process and twoprocess cylinder liner honed surfaces under reciprocating sliding conditions," Tribology International, 43(10), pp. 1882-1892.

[46] Vilhena, L. M., Podgornik, B., Vižintin, J., and Možina, J., 2011, "Influence of texturing parameters and contact conditions on tribological behaviour of laser textured surfaces," Meccanica, 46(3), pp. 567-575.

[47] Qiu, M., Delic, A., and Raeymaekers, B., 2012, "The effect of texture shape on the load-carrying capacity of gas-lubricated parallel slider bearings," Tribology Letters, 48(3), pp. 315-327.

[48] Bijani, D., Deladi, L. E., and Schipper, D. J., 2016, "The Influence of Surface Texturing on the Film Thickness in Parallel Sliding Surfaces," 20th International Colloquium Tribology: Technische Akademie Esslingen, p. 12.

[49] Patankar, S. V., 1980, Numerical heat transfer and fluid flow / Suhas V. Patankar, Hemisphere Pub. Corp. ; McGraw-Hill, Washington : New York.

[50] Versteeg, H. K., and Malalasekera, W., 1995, An Introduction to Computational Fluid Dynamics. The Finite Volume Method, Longman Group Ltd.

[51] Bijani, D., Deladi, E., de Rooij, M., and Schipper, D., 2018, "The influence of surface texturing on the film thickness in starved lubricated parallel sliding contacts," Lubricants, 6(3), p. 61.

[52] Glavatskih, S. B., McCarthy, D. M. C., and Sherrington, I., 2005, "Hydrodynamic Performance of a Thrust Bearing with Micropatterned Pads," Tribology Transactions, 48(4), pp. 492-498.

[53] Etsion, I., 2010, "State of the Art in Laser Surface Texturing," Advanced Tribology, J. Luo, Y. Meng, T. Shao, and Q. Zhao, eds., Springer Berlin Heidelberg, pp. 761-762.

[54] Etsion, I., and Burstein, L., 1996, "A Model for Mechanical Seals with Regular Microsurface Structure," Tribology Transactions, 39(3), pp. 677-683.

[55] Ronen, A., Etsion, I., and Kligerman, Y., 2001, "Friction-Reducing Surface-Texturing in Reciprocating Automotive Components," Tribology Transactions, 44(3), pp. 359-366.

[56] Pemberton, J., and Cameron, A., 1976, "A mechanism of fluid replenishment in elastohydrodynamic contacts," Wear, 37(1), pp. 185-190.

[57] Floberg, L., 1961, "Boundary conditions of cavitation regions in journal bearings," ASLE Transactions, 4(2), pp. 282-286.

[58] Dalmaz, G., and Godet, M., 1973, "Traction, load and film thickness in lightly-loaded lubruicated point contacts," Journal Mechanical Engineering Science, 15(6).

[59] Brewe, D., and Hamrock, B., 1982, "Analysis of starvation effects on hydrodynamic lubrication in nonconforming contacts," Trans. ASME, Journal Lubrication Technology, 104, pp. 410-417.

[60] Boness, R. J., 1970, "Effect of oil supply on cage and roller motion in a lubricated roller bearing.," J Lubric Technol Trans ASME, 92 Ser F(1), pp. 39-53.

[61] Yoshizawa, H., You-Lung, C., and Israelachvili, J., 1993, "Recent advances in molecular level understanding of adhesion, friction and lubrication," Wear, 168(1-2), pp. 161-166.

[62] Wang, S. Q., 1999, "Molecular transitions and dynamics at melt/wall interfaces: Origins of flow instabilities and wall slip," Adv. Polym. Sci., 138.

[63] Cottin-Bizonne, C., Barentin, C., Charlaix, É., Bocquet, L., and Barrat, J. L., 2004, "Dynamics of simple liquids at heterogeneous surfaces: Molecular-dynamics simulations and hydrodynamic description," Eur. Phys. J. E, 15(4), pp. 427-438.

[64] Ma, M., and Hill, R. M., 2006, "Superhydrophobic surfaces," Current Opinion in Colloid \& Interface Science, 11(4), pp. 193-202.

[65] Ybert, C., Barentin, C., Cottin-Bizonne, C., Joseph, P., and Bocquet, L., 2007, "Achieving large slip with superhydrophobic surfaces: Scaling laws for generic geometries," Physics of fluids, 19(12), p. 123601. 
[66] Hild, W., Opitz, A., Schaefer, J. A., and Scherge, M., 2003, "The effect of wetting on the microhydrodynamics of surfaces lubricated with water and oil," Wear, 254(9), pp. 871-875.

[67] Priezjev, N. V., Darhuber, A. A., and Troian, S. M., 2005, "Slip behavior in liquid films on surfaces of patterned wettability: Comparison between continuum and molecular dynamics simulations," Physical Review E, 71(4), p. 041608.

[68] Zhu, Y., and Granick, S., 2002, "No-Slip Boundary Condition Switches to Partial Slip When Fluid Contains Surfactant," Langmuir, 18(26), pp. 10058-10063.

[69] Spikes, H., and Granick, S., 2003, "Equation for Slip of Simple Liquids at Smooth Solid Surfaces," Langmuir, 19(12), pp. 5065-5071.

[70] Bijani, D., de Rooij, M., Deladi, E., and Schipper, D., "The Influence of Surface Texturing and Boundary slip on the Film Thickness in Parallel Sliding Surfaces," Proc. 20th International Colloquium Tribology, 20th International Colloquium Tribology.

[71] Tauviqirrahman, M., Ismail, R., Jamari, J., and Schipper, D. J., 2013, "Computational analysis of the lubricated-sliding contact with artificial slip boundary," International Journal of Applied Mathematics and Statistics, 35(5), pp. 67-80.

[72] Tauviqirrahman, M., Ismail, R., Jamari, J., and Schipper, D. J., 2013, "A study of surface texturing and boundary slip on improving the load support of lubricated parallel sliding contacts," Acta mechanica, 224(2), pp. 365-381.

[73] Choo, J. H., Spikes, H. A., Ratoi, M., Glovnea, R., and Forrest, A., 2007, "Friction reduction in lowload hydrodynamic lubrication with a hydrophobic surface," Tribology International, 40(2), pp. 154159.

[74] Hirst, W., 1974, "Scuffing and its prevention," Chart Mech Eng, 21(4), pp. 88-92.

[75] Ryk, G., and Etsion, I., 2006, "Testing piston rings with partial laser surface texturing for friction reduction," Wear, 261(7-8), pp. 792-796.

[76] Etsion, I., and Sher, E., 2009, "Improving fuel efficiency with laser surface textured piston rings," Tribology International, 42(4), pp. 542-547.

[77] Sęp, J., and Kucaba-Piętal, A., 2001, "Experimental testing of journal bearings with two-component surface layer in the presence of an oil abrasive contaminant," Wear, 249(12), pp. 1090-1095.

[78] Galda, L., Dzierwa, A., Sep, J., and Pawlus, P., 2010, "The Effect of Oil Pockets Shape and Distribution on Seizure Resistance in Lubricated Sliding," Tribology Letters, 37(2), pp. 301-311.

[79] Sep, J., Pawlus, P., and Dzierwa, A., "The analysis of abrasive wear of the helical grooved journal bearing," Proc. Key Engineering Materials, Trans Tech Publ, pp. 173-178.

[80] Bijani, D., Deladi, E., Akchurin, A., de Rooij, M., and Schipper, D., 2018, "The influence of surface texturing on the frictional behaviour of parallel sliding lubricated surfaces under conditions of mixed lubrication," Lubricants, 6(4), p. 91.

[81] Zhu, D., and Hu, Y.-Z., 2001, "A Computer Program Package for the Prediction of EHL and Mixed Lubrication Characteristics, Friction, Subsurface Stresses and Flash Temperatures Based on Measured 3-D Surface Roughness," Tribology Transactions, 44(3), pp. 383-390.

[82] Hu, Y. Z., and Zhu, D., 2000, "A full numerical solution to the mixed lubrication in point contacts," Journal of Tribology, 122(1), pp. 1-9.

[83] Zhu, D., Cheng, H. S., and Hamrock, B. J., 1990, "Effect of Surface Roughness on Pressure Spike and Film Constriction in Elastohydrodynamically Lubricated Line Contacts," Tribology Transactions, 33(2), pp. 267-273.

[84] Greenwood, J. A., and Williamson, J. B. P., 1966, "Contact of Nominally Flat Surfaces," Proceedings of the Royal Society of London. Series A. Mathematical and Physical Sciences, 295(1442), pp. 300319.

[85] Johnson, K. L., Greenwood, J. A., and Poon, S. Y., 1972, "A simple theory of asperity contact in elastohydro-dynamic lubrication," Wear, 19(1), pp. 91-108. 
[86] Faraon, I. C., 2005, "Mixed lubricated line contacts, PhD thesis,"PhD Thesis, University of Twente, Enschede. 
Part II 



\section{Part II}

Paper A: Dariush Bijani, E.L. Deladi and D.J. Schipper. The Influence of Surface Texturing on the Film Thickness in Parallel Sliding Surfaces. TAE 2016 Proceedings, ISBN-Nr. 978-3-94356323-8

Paper B: Dariush Bijani, Elena L. Deladi, Matthijn B. de Rooij and Dirk J. Schipper. The Influence of Surface Texturing on the Film Thickness in Starved Lubricated Parallel Sliding Contacts. Lubricants 2018, 6(3), 61; https://doi.org/10.3390/lubricants6030061

Paper C: Dariush Bijani, Elena L. Deladi, Aydar Akchurin, Matthijn B. de Rooij and Dirk J. Schipper. The Influence of Surface Texturing on the Frictional Behaviour of Parallel Sliding Lubricated Surfaces under Conditions of Mixed Lubrication. Lubricants 2018,6(4), 91; https://doi.org/10.3390/lubricants6040091

Paper D: Dariush Bijani, Elena L. Deladi, Matthijn B. de Rooij and Dirk J. Schipper. The influence of surface texturing on the frictional behaviour in starved lubricated parallel sliding contacts. Lubricants 2019, 7(8), 68; https://doi.org/10.3390/lubricants7080068

Paper E: Dariush Bijani, M.B. de Rooij, E.L. Deladi and D.J. Schipper. The influence of surface texturing and boundary slip on the film thickness in parallel sliding surfaces. TAE 2018 Proceedings, ISBN-Nr. 978-3-943563-33-7

Paper F: Dariush Bijani, M.B. de Rooij, G.R.B.E. Römer, E.L. Deladi and D.J. Schipper, An experimental study on the influence of surface texturing in flat on flat lubricated contacts. Tribology letters 2019 (Article in press) 



\title{
Paper A
}

\section{The Influence of Surface Texturing on the Film Thickness in Parallel Sliding Surfaces}

\author{
Dariush Bijani, D.J. Schipper and E.L. Deladi
}

TAE 2016 Proceedings

The Influence of Surface Texturing on the Film Thickness in Parallel Sliding Surfaces

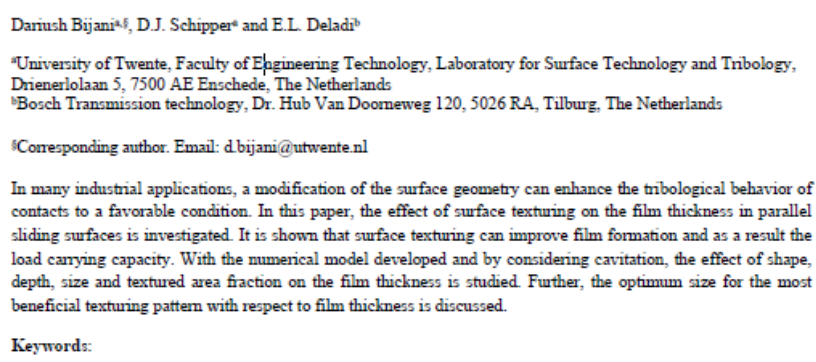

1- Introduction

Suface textuing for enhancing tribological properties of mechanical components is well known for many years. This technigue has emerged in the last decade as viable option to improve the load canying capacity, wear resistance, and reduce the firction coefficient of Whicated systems. It is possible by using an optimized to retain lubricant and enhance the hydrodynamic effect [1-9]

Micro-geometric cavities have been successfully used to mprove the lubrication between surfaces. By applyin a texture at the surface, the lubricating fluid is pulled into the converging gap formed by the texture when the suffaces slide related to each other, which generates pressure in the fluid. These micro-scale cavities can act s micro-reservoirs, can entrap wear particles, enhance the load canying capacity and increase the fil hickners, see for instance Etrion et al $[2,5,6]$ Sufface exturing influences also the transitions between the lubrication regimes [7].

In work of Ryk et al. [9] the potential benefits of applying LST (laser surface texturing) to piston ring have been demonstrated theoretically and experimentally. Optimum texturing parameters for the inimum fiction force were found for full LST ring Good agreement was found with experimental results.
The results show the benefits of LST in both full and starved lubrication conditions. The use of laser texturing in the form of micro-grooves on cylinder liners of intermal combustion engnes show lower fuel consumption and wear. The textured sider provides an effective larger film thickness than the non-texture portion and hence, the slider is actung as a Rayleigh step bearing. Test results showed that the textured bearng operated with a film thickness that is about 3 times the film thickness of the nontextured beanng throughout the range of tested loads. In work of Vilhena et al. [10], the performance of a reference non-textured surface and extured dises with twee different dimple depths are compared. The study is based on experiments. They obtained that the beneficial effect of micro-dimples becomes larger with an increase in dimple depth.

In the work of Yu et al. [11] a theoretical model based on a single dimple was established to investigate the geometric shape and onientation effects of the curcular, elliptical and trangular pockets on the hydrodynami pressure generated between conformal contacting surfaces. Their results indicate that the geometric shape and orientation influences the load-carrying capacity of the contacting surfaces. With the same dimple area, area ratio and dimple depth the ellipse haped dimples with the major axis pependicula to the sliding direction showed the best loadcarrying capacity among all cases. 



\title{
The Influence of Surface Texturing on the Film Thickness in Parallel Sliding Surfaces
}

\author{
Dariush Bijani ${ }^{a}{ }^{\S}$, D.J. Schipper ${ }^{a}$ and E.L. Deladi ${ }^{b}$ \\ a University of Twente, Faculty of Engineering Technology, Laboratory for Surface Technology and Tribology, \\ Drienerlolaan 5, 7500 AE Enschede, The Netherlands

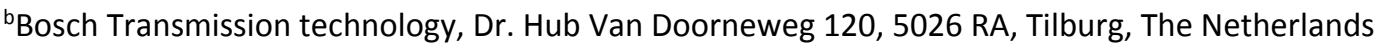 \\ ${ }^{\S}$ Corresponding author. Email: d.bijani@utwente.nl \\ In many industrial applications, a modification of the surface geometry can enhance the tribological behaviour \\ of contacts to a favourable condition. In this paper, the effect of surface texturing on the film thickness in parallel \\ sliding surfaces is investigated. It is shown that surface texturing can improve film formation and, as a result, the \\ load-carrying capacity. With the numerical model developed and by taking cavitation into account, the effect of \\ shape, depth, size and textured area fraction on the film thickness is studied. Further, the optimum size for the \\ most beneficial texturing pattern with respect to film thickness is discussed.
}

\section{Keywords:}

Hydrodynamic lubrication; surface texturing; film thickness; texturing patterns, numerical modelling

\section{Introduction}

Surface texturing for enhancing tribological properties of mechanical components has been well known for many years. This technique has emerged in the last decade as a viable option to improve the load-carrying capacity and wear resistance, and to reduce the friction coefficient of lubricated systems. Using an optimized texture it is possible to retain lubricant and enhance the hydrodynamic effect [1-9].

Micro-geometric cavities have been used successfully to improve the lubrication between surfaces. By applying a texture at the surface, the lubricating fluid is pulled into the converging gap formed by the texture when the surfaces slide relative to each other, which generates a pressure in the fluid. These micro-scale cavities can act as micro-reservoirs, can entrap wear particles, enhance the load-carrying capacity and increase the film thickness, see for instance Etsion et al. $[2,5,6]$. Surface texturing also influences the transitions between the lubrication regimes [7].

In the work of Ryk et al. [9], the potential benefits of applying LST (laser surface texturing) to piston rings have been demonstrated theoretically and experimentally. Optimum texturing parameters for the minimum friction force were found for full LST rings. Good agreement was found with experimental results. The results show the benefits of LST in both full and starved lubrication conditions. The use of laser texturing in the form of micro-grooves on cylinder liners of internal combustion engines shows lower fuel consumption and wear. The textured slider provides an effective larger film thickness than the non-textured portion, hence the slider is acting as a Rayleigh step bearing. Test results showed that the textured bearing operated with a film thickness that is about three times the film thickness of the non-textured bearing throughout the range of tested loads. In the work of Vilhena et al. [10], the performance of a reference nontextured surface and textured discs with three different dimple depths are compared. The study is based on experiments. The results they obtained indicate that the beneficial effect of micro-dimples becomes larger with an increase in dimple depth. 
In the work of Yu et al. [11], a theoretical model based on a single dimple was established to investigate the geometric shape and orientation effects of the circular, elliptical and triangular pockets on the hydrodynamic pressure generated between conformal contacting surfaces. Their results indicate that the geometric shape and orientation influences the load-carrying capacity of the contacting surfaces. With the same dimple area, area ratio and dimple depth the ellipse shaped dimples with the major axis perpendicular to the sliding direction showed the best load-carrying capacity of all.

In a study by Kovalchenko et al. [7], the influence of texturing on the transitions between the different lubricating regimes was investigated. They observed that LST is able to extend the range of the hydrodynamic lubrication regime in terms of load and sliding velocity. Removal of the bulges at the edges of dimples by lapping after laser texturing is essential in optimizing the positive effect of LST.

In the study by Ren et al. [12], the focus of the study was on the selection of texture distribution patterns based on their lubrication performance. The effects of the cavity aspect ratio were also studied. The results indicate that, for the given material and geometry of the system under the given conditions, the textures generating the highest hydrodynamic load-carrying capacity are short grooves with a small aspect ratio perpendicular to the direction of motion They found that the cavity aspect ratio in terms of width/length is related inversely to the film thickness.

In the work of Costa and Hutchings [13], the influence of surface topography on lubricant film for the reciprocating sliding situation was studied. In this investigation, a textured steel surface against cylindrical counterbodies was studied. Several patterns like grooves and chevrons were used, and parameters like the textured area fraction, depth, width and sliding direction on the textured surface were studied. The highest values of the film thickness were found in the sample with medium depth/width ratio. Thus, when the ratio becomes too large, the load-carrying capacity reduces. They demonstrated that, among the investigated patterns, chevrons were the most effective and grooves were the least effective in order to increase the hydrodynamic film thickness. Chevron patterns pointing along the sliding direction gave a greater film thickness than those pointing across it.
In 1886, Osborne Reynolds presented a partial differential equation to describe pressure build-up in self-acting bearings. This equation has been used successfully to determine the pressure distribution in the fluid film for a wide range of applications from bearings and seals to metal forming processes. This equation is deduced from the Navier-Stokes equations under the assumptions that the gap between the surfaces and the Reynolds number is small. The Reynolds equation in Cartesian coordinates can be written as (Eq. 1):

$$
\begin{aligned}
\frac{\partial}{\partial x}\left(\frac{\rho h^{3}}{\eta}\right. & \left.\frac{\partial p}{\partial x}\right)+\frac{\partial}{\partial y}\left(\frac{\rho h^{3}}{\eta} \frac{\partial p}{\partial y}\right) \\
& =6\left(\mathrm{u}_{0}\right) \frac{\partial(\rho h)}{\partial x}+6 \rho h \frac{\partial\left(\mathrm{u}_{0}\right)}{\partial x} \\
& +12 \frac{\partial(\rho h)}{\partial t}
\end{aligned}
$$

At the outlet of the cavity, the lubricant is dragged through a converging region and, as a result, pressure is generated. At the entry of the cavity, the lubricant flow diverges which results, according to the Reynolds equation (Eq. 1), in a negative pressure. However, negative pressure is suppressed by cavitation at which vapour bubbles are formed in the lubricant.

For moderately loaded lubricated systems, the Jakobsson-Floberg-Olsson [14, 15] cavitation theory is used, which represents a reasonable degree of accuracy. It is not suitable for cases where surface tension plays an important role, such as in face seals [16]. Based on Jakobsson-Floberg-Olsson, the lubrication film is divided into two zones. The first part is with a complete lubricant film, in which the pressure varies, and in this region the Reynolds equation applies. In the second part, cavitation occurs and only a fraction of the lubricant film gap is occupied. Because of the intervening gas within the void fraction, the pressure throughout the cavitation area is taken as constant [16].

According to the Payvar-Salant model $[17,18]$, the steady-state mass-conservation Reynolds equation, taking cavitation into account, can be written in a Cartesian coordinate system as (Eq. 2) [19]:

$$
\begin{aligned}
& \frac{\partial}{\partial x}\left(\frac{h^{3}}{\eta} \frac{\partial(F \phi)}{\partial x}\right)+\frac{\partial}{\partial y}\left(\frac{h^{3}}{\eta} \frac{\partial(F \phi)}{\partial y}\right) \\
= & \frac{6 u_{0}}{p_{a}-p_{c}} \frac{\partial((1+(1-F) \varphi) h)}{\partial x}
\end{aligned}
$$

In this equation $h$ is the film thickness, $\eta$ is the viscosity, $u_{0}$ is the sum velocity, $p_{a}$ is the ambient pressure, $p_{c}$ is the cavitation pressure, $\varphi$ is a 
dimensionless dependent variable and $F$ is the cavitation index. For more information the reader is referred to Wang [19].

Dobrica et al. [20] carried out an investigation based on the influence of cavitation and different texturing parameters on the hydrodynamic performance of textured contacts. Their results are derived numerically, based on the Reynolds equation with the JFO formulation on the plane-parallel contact and the sliding inclined contact. They studied the optimal textured region and the optimal dimple aspect ratio. In their investigation, cavitation was shown to have a significant influence on the performance of planeparallel textured sliding contacts. In partially textured parallel sliders, cavitation had a positive effect by increasing the inlet flow (inlet suction). In convergent sliders with a high incline ratio, texturing showed a minimal effect as well as cavitation. In their study the optimal dimple depth and length were determined for different plane-inclined sliders.

Based on the Reynolds equation, film thickness equations are developed for several configurations. The minimum film thickness in hydrodynamically lubricated contacts is a function of the applied normal applied load $F_{n}$ (Contact pressure- $P_{a}$ ), sum velocity $u_{0}$, lubricant viscosity $\eta$ and macro geometry. The film thickness as a function of these parameters is given in (Eq. 3):

$$
\mathrm{h}_{H L} \propto \mathrm{G}\left(\frac{\eta u_{0}}{\mathrm{P}_{a}}\right)^{1 / 2}
$$

in which $\mathrm{P}_{a}$ is the average contact pressure and $\mathrm{G}$ is the geometry parameter.

In this paper the effect of texturing (meso-geometry), including cavitation on the film formation, is studied for macroscopically plane-parallel sliding surfaces. The surfaces are smooth, i.e. no roughness (microgeometry).

\section{Mathematical modelling and solution:}

\section{Cavity shape and geometry:}

Four different texture patterns have been investigated in this study: circular pocket, triangular pocket, chevron and groove. Figure 1 shows the different cavity shapes, and the parameters characterizing their geometry. The chevron pattern is defined by two similar equilateral triangles of different sizes. The triangular pocket is a special case of the chevron with the inner edge length of the chevron equal to zero. For these two cases, the centre of the unit cell coincides with the midpoint of the altitude line of the triangle or chevron shape, see also [21]. All patterns have a rectangular cross-sectional profile. The general film thickness formula can be written as (Eq. 4):

$$
h=h_{0}+h_{\text {macro }}+h_{\text {texture }}
$$

In the case of flat-flat contact, the macro geometry is omitted and the film thickness (Eq. 4) reduces to (Eq. 5) $[21,22]$ :

$$
\frac{h(x, y)}{h_{0}(x, y)}=1+H(x, y)
$$
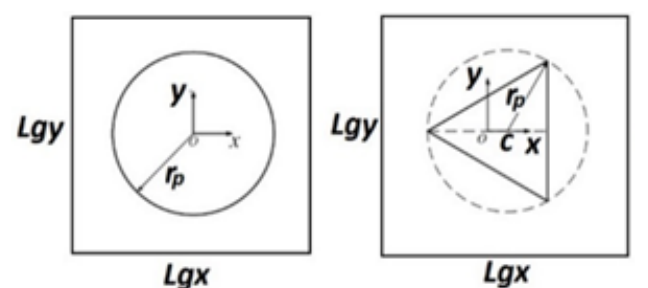

(a)
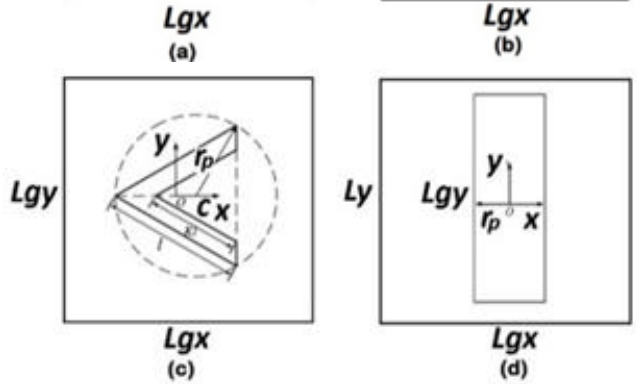

Figure 1. Geometrical scheme of patterns, a) Circular pocket, b) Triangular pocket, c) Chevron and d) Groove [21].

In this simulation $r_{p}$ is characteristic radius for triangular, circular and chevron patterns and half width of grooves.

The film thickness formula for the circular pocket is given in (Eq. 6):

$$
H(x, y)=\left\{\begin{array}{cc}
0, & X^{2}+Y^{2}>1 \\
\frac{T_{d}}{h_{0}}, & X^{2}+Y^{2} \leq 1
\end{array}\right.
$$

The film thickness formula for the triangular pocket can be written as (Eq. 7):

$$
\begin{gathered}
H(x, y)=\left\{\begin{array}{cc}
0, & (X, Y) \notin \Omega \\
\frac{T_{d}}{h_{0}}, \quad & (X, Y) \in \Omega
\end{array}\right. \\
\Omega:-\frac{3}{4} \leq X \leq \\
\frac{3}{4} \text { and }\left\{\begin{array}{c}
\frac{1}{\sqrt{3}} X-\frac{\sqrt{3}}{4} \leq Y \leq \frac{1}{\sqrt{3}} X+\frac{\sqrt{3}}{4} \\
-\frac{1}{\sqrt{3}} X-\frac{\sqrt{3}}{4} \leq Y \leq-\frac{1}{\sqrt{3}} X+\frac{\sqrt{3}}{4}
\end{array}\right.
\end{gathered}
$$

The film thickness formula for the chevron can be written as (Eq. 8): 


$$
\begin{gathered}
H(x, y)=\left\{\begin{array}{cc}
0, & (X, Y) \notin \Omega \\
\frac{T_{d}}{h_{0}}, \quad & (X, Y) \in \Omega \\
\Omega:-\frac{3}{4} \leq X
\end{array}\right. \\
\leq \frac{3}{4} \text { and }\left\{\begin{array}{c}
\frac{1}{\sqrt{3}} X+\frac{\sqrt{3}}{2}\left(K-\frac{1}{2}\right) \leq \\
Y \leq \frac{1}{\sqrt{3}} X+\frac{\sqrt{3}}{4} \\
-\frac{1}{\sqrt{3}} X-\frac{\sqrt{3}}{4} \leq \\
Y \leq-\frac{1}{\sqrt{3}} X+\frac{\sqrt{3}}{2}\left(\frac{1}{2}-K\right)
\end{array}\right.
\end{gathered}
$$

The film thickness formula for the grooves is given in (Eq. 9):

$$
\begin{gathered}
H(x, y)= \begin{cases}0, & (X, Y) \notin \Omega \\
\frac{T_{d}}{h_{0}}, & (X, Y) \in \Omega\end{cases} \\
\Omega:-\frac{1}{2} \leq X \leq \frac{1}{2} \text { and } \frac{1}{2} \leq Y \leq \frac{1}{2}
\end{gathered}
$$

In order to discretize the Reynolds equation, a control volume is used to solve the pressure at point $P$ in terms of the pressures at the points N, E, S and W (see Fig. 2). Integrating (Eq. 2) over the control volume:

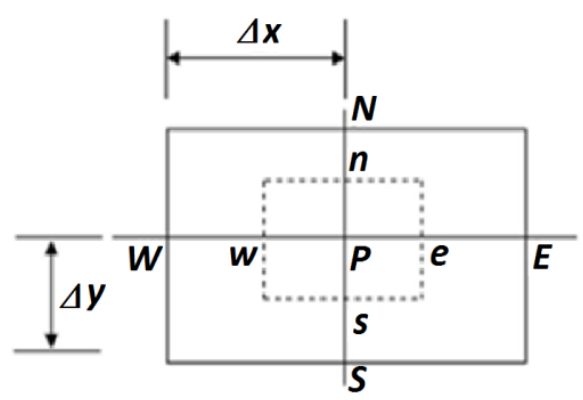

Figure 2. Control volume around node $\mathbf{P}$.

$$
\begin{aligned}
k_{n} \frac{\Delta x_{i j}}{(\delta y)_{n}} & F_{N} \varphi_{N}+k_{s} \frac{\Delta x_{i j}}{(\delta y)_{s}} F_{S} \varphi_{S} \\
& +k_{e} \frac{\Delta y_{i j}}{(\delta x)_{e}} F_{E} \varphi_{E} \\
& +k_{w} \frac{\Delta y_{i j}}{(\delta x)_{w}} F_{W} \varphi_{W} \\
& -\left\{\left(k_{n} \frac{\Delta x_{i j}}{(\delta y)_{n}}+k_{s} \frac{\Delta x_{i j}}{(\delta y)_{s}}\right.\right. \\
& +k_{e} \frac{\Delta y_{i j}}{(\delta x)_{e}} \\
& \left.\left.+k_{w} \frac{\Delta y_{i j}}{(\delta x)_{w}}\right) F_{P}\right\} \varphi_{P} \\
& =\left(\frac{6 u_{0}}{p_{a}-p_{c}}\right)\{(1 \\
& \left.+\left(1-F_{P}\right) \varphi_{P}\right) h_{e} \\
& \left.-\left(1+\left(1-F_{W}\right) \varphi_{W}\right) h_{w}\right\} \Delta y_{i j}
\end{aligned}
$$

The derivatives in (Eq. 10) can be approximated by: $k=\frac{h^{3}}{\eta}$,

$(\delta x)_{w}=x_{P}-x_{W}$

$(\delta x)_{e}=x_{E}-x_{P}$

$(\delta y)_{S}=y_{P}-y_{S}$,

$(\delta y)_{n}=y_{N}-y_{P}$

$\Delta x_{i j}=\left((\delta x)_{w}+(\delta x)_{e} / 2\right)$ and $\Delta y_{i j}$

$$
=\left((\delta y)_{s}+(\delta y)_{n} / 2\right)
$$

Identifying the coefficients of $\varphi_{E}, \varphi_{W}, \varphi_{N}$ and $\varphi_{S}$ as $A_{E}, A_{W}, A_{N}$ and $A_{S}$, and the coefficient of $\varphi_{P}$ as $A$, the above equation can be written as (Eq. 11):

$A_{N} \varphi_{N}+A_{S} \varphi_{S}+A_{E} \varphi_{E}+A_{W} \varphi_{W}-A_{P} \varphi_{P}=B_{P}$

Which:

$$
\begin{aligned}
A_{N} & =k_{n} \frac{\Delta x_{i j}}{(\delta y)_{n}} F_{N}, \\
A_{S} & =k_{s} \frac{\Delta x_{i j}}{(\delta y)_{s}} F_{S}, \\
A_{E} & =k_{e} \frac{\Delta y_{i j}}{(\delta x)_{e}} F_{E}, \\
A_{W} & =k_{w} \frac{\Delta y_{i j}}{(\delta x)_{w}} F_{W}+\left(\frac{6 u_{0}}{p_{a}-p_{c}}\right)\left(1-F_{W}\right) h_{W} \Delta y_{i j}
\end{aligned}
$$

In which $A_{P}$ is the weighted arithmetic average of the other coefficients and can be written as (Eq. 12):

$$
\begin{aligned}
A_{P}=\left(k_{n} \frac{\Delta x_{i j}}{(\delta y)_{n}}+k_{s} \frac{\Delta x_{i j}}{(\delta y)_{s}}+k_{e} \frac{\Delta y_{i j}}{(\delta x)_{e}}\right. \\
\left.+k_{w} \frac{\Delta y_{i j}}{(\delta x)_{w}}\right) F_{P} \\
\quad+\left(\frac{6 u_{0}}{p_{a}-p_{c}}\right)\left(1-F_{P}\right) h_{e} \Delta y_{i j}
\end{aligned}
$$

For the source term of the equation:

$$
B_{P}=\left(\frac{6 u_{0}}{p_{a}-p_{c}}\right)\left(h_{e}-h_{w}\right) \Delta y_{i j}
$$


In order to compute the coefficient $K$ in the centre of each unit cell interpolation is used. In this case, the harmonic mean value scheme on each lattice of the boundary is applied and is given in (Eq. 14):

$$
\begin{gathered}
k_{n}=\frac{2}{\frac{1}{K_{N}}+\frac{1}{K_{P}}}, k_{s}=\frac{2}{\frac{1}{K_{S}}+\frac{1}{K_{P}}}, \\
k_{e}=\frac{2}{\frac{1}{K_{E}}+\frac{1}{K_{P}}}, k_{w}=\frac{2}{\frac{1}{K_{W}}+\frac{1}{K_{P}}} \\
k_{P}=\frac{h_{i, j}^{3}}{\eta_{i, j}}, \\
k_{N}=\frac{h_{i, j+1}^{3}}{\eta_{i, j+1}}, k_{S}=\frac{h_{i, j-1}^{3}}{\eta_{i, j-1}} \\
k_{E}=\frac{h_{i+1, j}^{3}}{\eta_{i+1, j}}, k_{W}=\frac{h_{i-1, j}^{3}}{\eta_{i-1, j}}
\end{gathered}
$$

To solve (Eq. 12) iterative solvers include nodal iteration, the tri-diagonal matrix algorithm (TMDA) is used and for reducing the storage needed for calculation the line-by-line TDMA solver (Patankar [23]) is applied. When one solves a two-dimensional problem, the TDMA solution column-by-column or row-by-row becomes iterative, and sweeping is done line-by-line and column-by-column or row-by-row. For three-dimensional problems the TDMA is applied lineby-line on a selected plane and then the calculation is moved to the next plane, scanning the domain planeby-plane [24].

\section{Results and discussion}

Several simulations are carried out in order to study the texturing patterns and to find the most efficient patterns and the effect of pattern orientation in the case of chevrons and triangular pockets.

The film thickness for chevrons and triangles parallel to the moving direction and perpendicular to the moving direction is calculated. The number and distance between the pockets are combined by introducing a new parameter, Pitch. The pitch for the chevron, triangular and circular pockets is calculated as:

Pitch in $x$ direction $=\frac{2 r_{p}}{L_{g x}}$

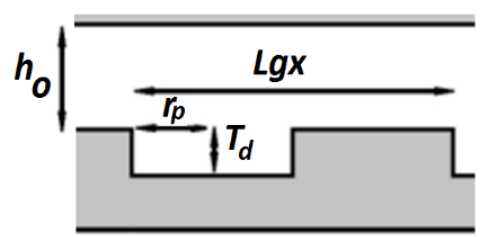

Figure 3. Schematic illustration of cavity profile.
The performance is investigated using a textured area with the following geometrical data:

Table 1. Texturing properties.

\begin{tabular}{c|c} 
Profile type & Rectangular cross-section (Fig. 3) \\
\hline Texture depth & $10 \mu \mathrm{m}$ \\
\hline Texture pitch & 0.4 \\
\hline$r_{p}$ & $50 \mu \mathrm{m}$ \\
\hline Normal load & $10 \mathrm{~N}$ \\
\hline Average pressure & $0.1 \mathrm{MPa}$ \\
\hline Lubricant viscosity & $0.008 \mathrm{~Pa} . \mathrm{S}$
\end{tabular}

The results are presented in (Fig. 5): the greatest film thickness is achieved for grooves perpendicular to the moving direction and the smallest film thickness is found when the chevron pattern is oriented perpendicular to the sliding direction (Fig. 4-f). The chevrons parallel to the sliding direction (Fig. 4-e) are more efficient than the circular pockets and triangular pockets (Fig. 4-c). Fig. 5 shows that the difference for triangular pockets parallel to the sliding direction and the pockets perpendicular to the moving direction is negligible and has the same values.

When the distances between texture cells are the same, i.e. pitch is constant, the groove pattern is more successful in generating local load-carrying capacity because of the higher textured area fraction per unit area. Chevrons in $\mathrm{x}$-direction are more beneficial than the triangular pockets and circular pockets because of the existence of a longer outlet wall in the cavity zone. Chevrons in Y-direction are less successful in this case because of the shorter distance between the flow inlet wall and outlet wall. 


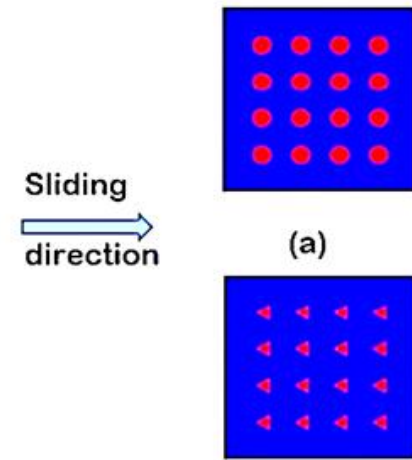

(c)

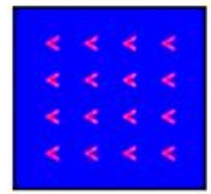

(e)

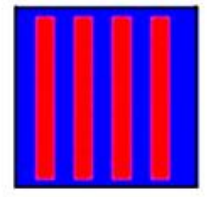

(b)

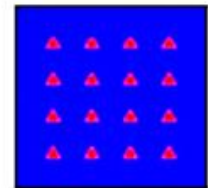

(d)

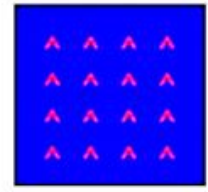

(f)
Figure 4. Schematic illustration of different patterns.

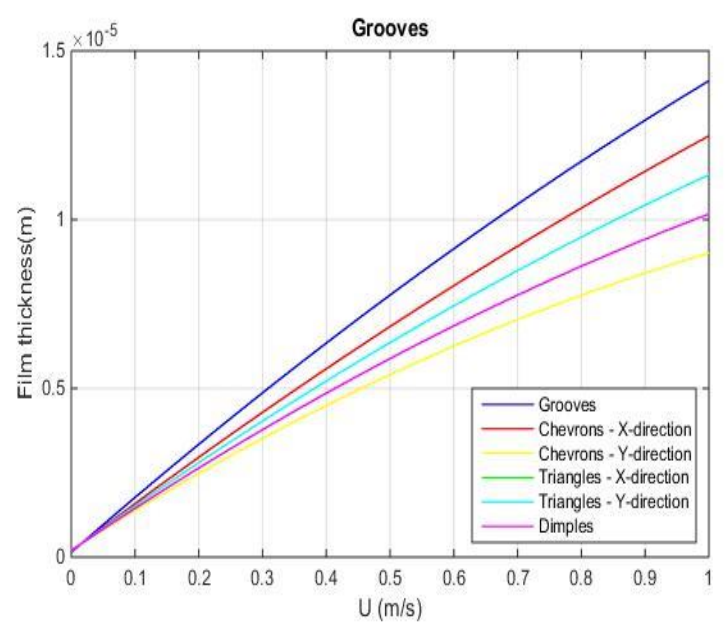

Figure 5. Comparison between different patterns, the triangular patterns are on top of each other.

\section{Cavity profile}

In this study, the influence of the cross-section of groove texturing is investigated. Simulation results based on grooves with a circular cross-section are compared with grooves with a rectangular crosssection. The shapes of these grooves are shown in Fig. 6. Textured areas with the following geometrical data have been selected to investigate its performance:

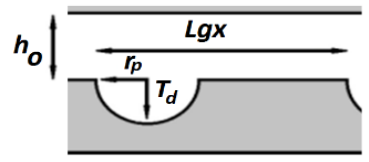

(a)

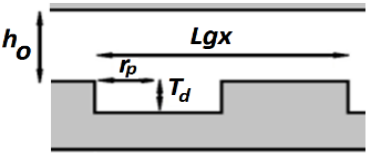

(b)
Figure 6. Schematic illustration of cavity profiles, a) Circular, b) Rectangular
Table 2. Texturing properties.

\begin{tabular}{c|c} 
Texture depth & $10 \mu \mathrm{m}$ \\
\hline Texture pitch & 0.4 \\
\hline$r_{p}$ & $50 \mu \mathrm{m}$ \\
\hline Normal load & $10 \mathrm{~N}$ \\
\hline Average pressure & $0.1 \mathrm{MPa}$ \\
\hline Lubricant viscosity & $0.008 \mathrm{~Pa} . \mathrm{s}$.
\end{tabular}

In (Fig. 7), the results of these simulations show that the grooves with a rectangular cross-section (Fig. 6-b) are more beneficial than those with the circular crosssection (Fig. 6-a). The main reason for this is the fact that a rectangular cross-section generates a higher pressure.

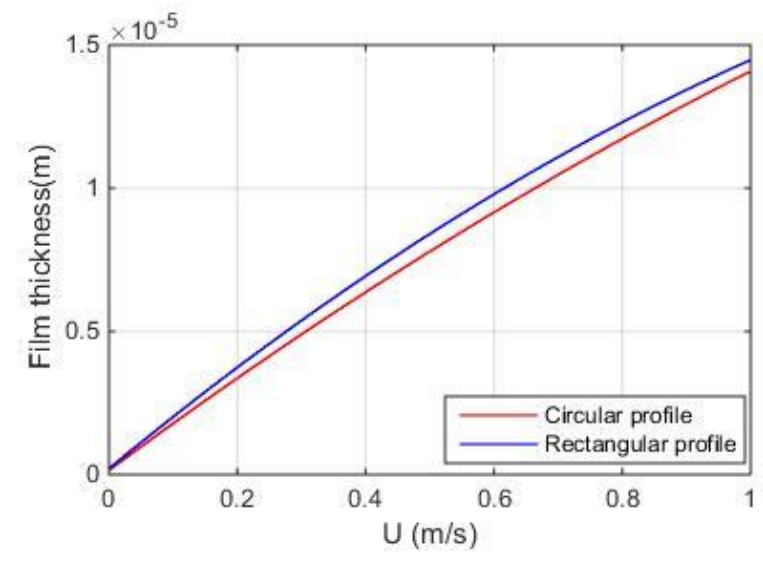

Figure 7. Effect of cross-sectional shape of grooves on film thickness.

\section{Open versus closed grooves}

Side leakage when using grooves, can have a significant effect in optimizing the texturing parameters, therefore grooves open at the sides are compared with a closed groove pattern (see Fig. 8). The pressure profile is presented in Fig. 9. The simulation results (Fig. 10) show the negative effect of side leakage.

The textured area with the following input geometrical data has been selected to investigate and compare its performance:

Table 3. Texture properties.

\begin{tabular}{c|c} 
Profile type & Circular (Fig. 6-a) \\
\hline Texture depth & $10 \mu \mathrm{m}$ \\
\hline Texture pitch & 0.4 \\
\hline$r_{p}$ & $45 \mu \mathrm{m}$ \\
\hline Normal load & $10 \mathrm{~N}$ \\
\hline Average pressure & $12 \mathrm{kPa}$ \\
\hline Lubricant viscosity & $0.008 \mathrm{~Pa} . \mathrm{s}$.
\end{tabular}


In this calculation $L_{g y} / L_{y}$ is equal to 0.85 .

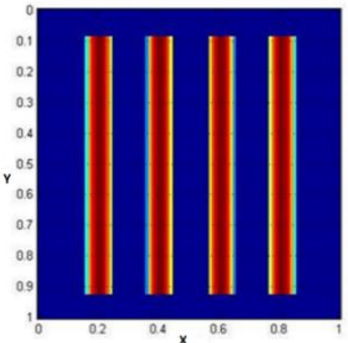

(a)

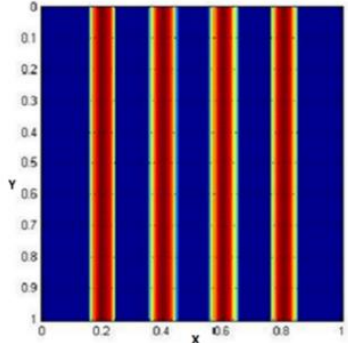

(b)
Figure 8. Schematic illustration of: a) Closed grooves, b) Open grooves.

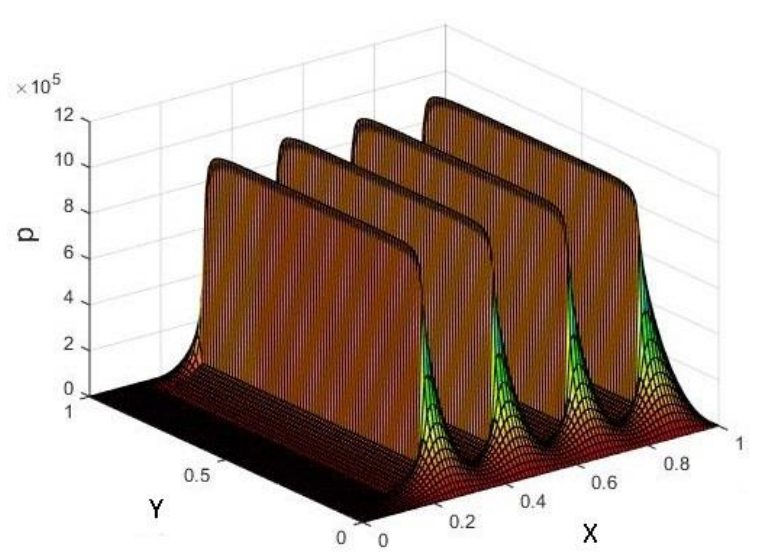

(a)

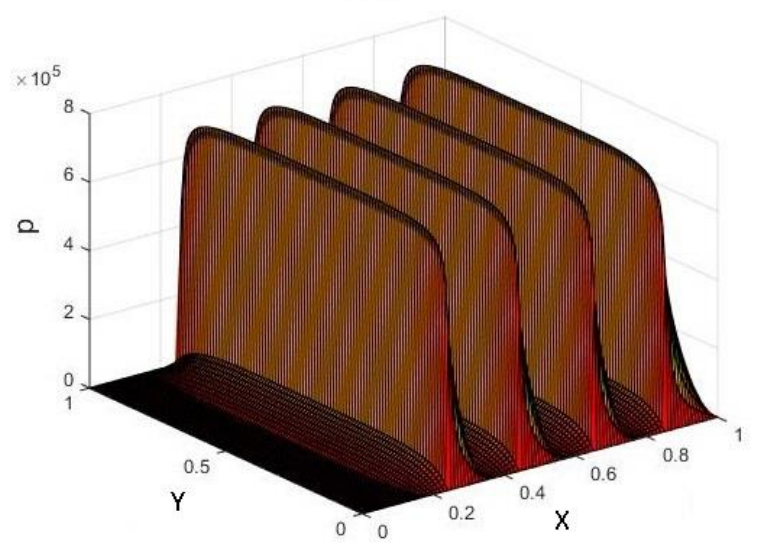

(b)

Figure 9. 3D Pressure profile, a) Open groove, b) Closed groove.

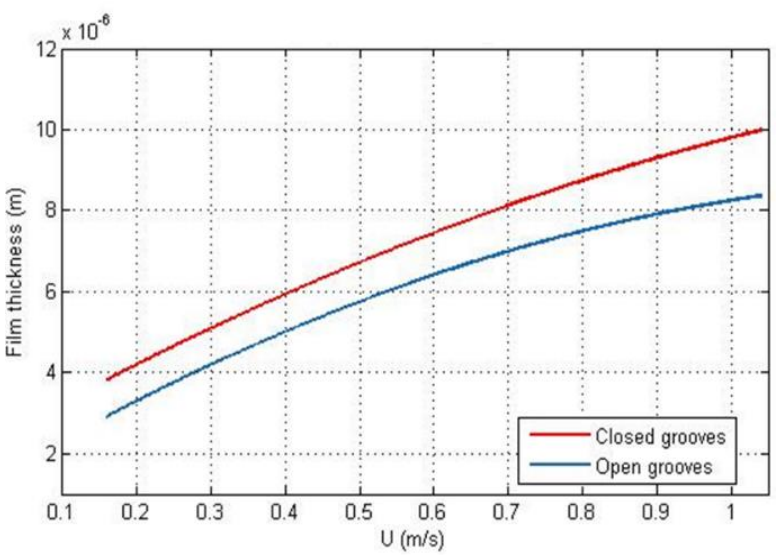

Figure 10. Film thickness for open and closed grooves.

In (Fig. 9), the results of these simulations show that it is possible to generate a higher pressure by applying the closed grooves. The higher generation is achievable by closed grooves because the effect of side leakage in open grooves is greater than in closed grooves. Therefore, a higher film thickness is achievable by applying the closed grooves, see Figure 10.

\section{Staggered versus normal array circular pockets}

Simulations have also been carried out to investigate the effect of how the pockets are arranged. In these simulations, the film thickness for two types of arrays is calculated. In Fig. 11-a, the pockets are arranged in such a way that the distance between the cavities in both directions is the same. In the second arrangement, pockets are shifted by half of the distance between two pockets in Y-direction to have a staggered array, as shown in Fig. 11-b.

Textured area with the following geometrical data has been selected for these calculations:

Table 4. Texturing properties.

\begin{tabular}{c|c} 
Profile type & Circular (Fig. 6-a) \\
\hline Texture depth & $10 \mu \mathrm{m}$ \\
\hline Texture pitch & 0.4 \\
\hline$r_{p}$ & $45 \mu \mathrm{m}$ \\
\hline Normal load & $10 \mathrm{~N}$ \\
\hline Average pressure & $12 \mathrm{kPa}$ \\
\hline Lubricant viscosity & $0.008 \mathrm{~Pa} . \mathrm{s}$
\end{tabular}




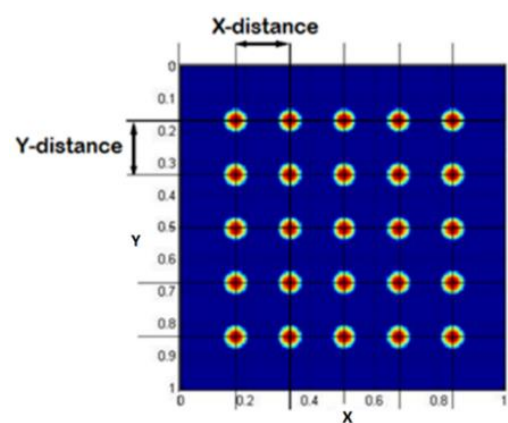

(a)

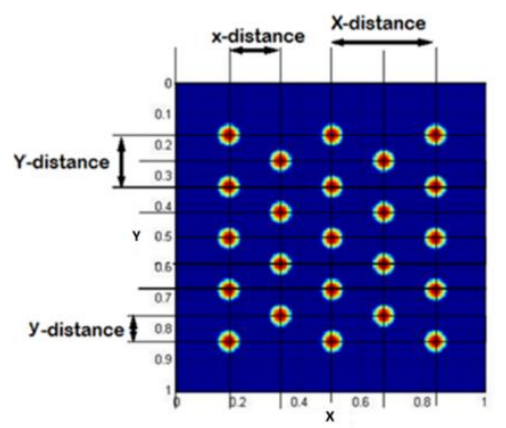

(b)

Figure 11. Different arrangement of pockets: a) normal array, b) staggered array.

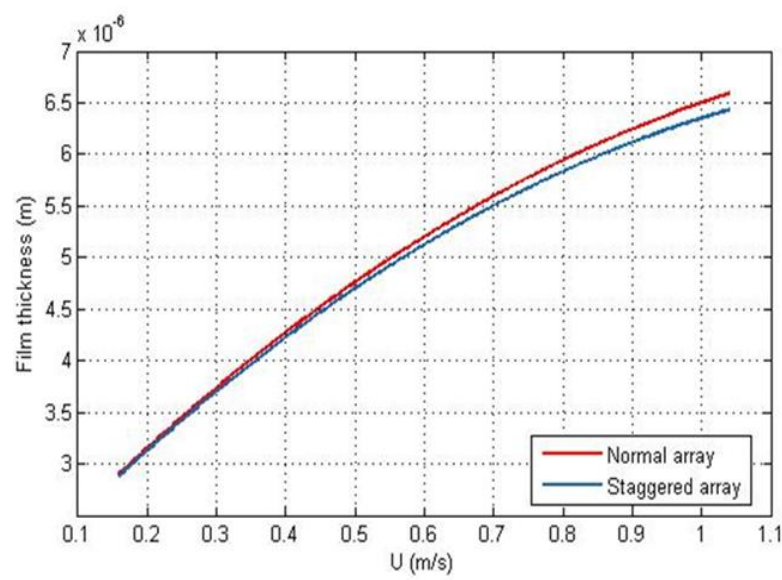

Figure 12. Effect of array type of circular pockets on the film thickness.

A comparison of the effect of different circular pocket arrangements on the film thickness is presented in Fig. 12. The results show that in this case the film thickness is not particularly sensitive to the arrangement of the circular pockets. Because of the existence of a higher textured area fraction in the case of the normal array of pockets, there is a slight difference over the film thickness, which ends towards the normal array of circular pockets.

Optimum texture properties:

In order to gain the benefits of texturing surfaces an optimization of the geometrical parameters is performed. In this optimization, the effect of textured area fraction and cavity geometrical ratio on film thickness based on pockets with a rectangular profile (Fig. 6-b) is studied. The textured area operates under an average pressure $5 \mathrm{MPa}$ and the lubricant viscosity is 0.008 Pa.s.

$S_{p}$ (Textured area fraction): Cavity area/Cell area,

$\xi$ (Cavity geometrical ratio): Cavity depth/Cavity length. It is possible to define the geometry of circular and triangular pockets by these two parameters:

For the circular pockets, these parameters can be written as (Eq. 15):

$$
\xi=\frac{T_{d}}{2 r_{p}}, \quad S_{p}=\frac{\pi r_{p}^{2}}{L_{g x} L_{g y}}
$$

For the triangular pockets (Eq. 16):

$$
\xi=\frac{T_{d}}{2 r_{p}}, \quad S_{p}=\frac{3 \sqrt{3} r_{p}^{2}}{4 L_{g x} L_{g y}}
$$

In Fig. 13, the simulation results show an optimum film thickness based on the texture area fraction $S_{p}$. When $S_{p}$ is tending to zero there is no film formation because of the absence of the wedge effect in flat-flat contact and the lack of texturing, therefore there is no pressure generation.

In order to analyze the cavity geometrical ratio simulations for the circular and the triangular pockets on $S_{p}$ the cavity geometrical ratio is taken as equal to 0.4 and to study the effect of textured area fraction the cavity geometrical ratio is 0.1 . 


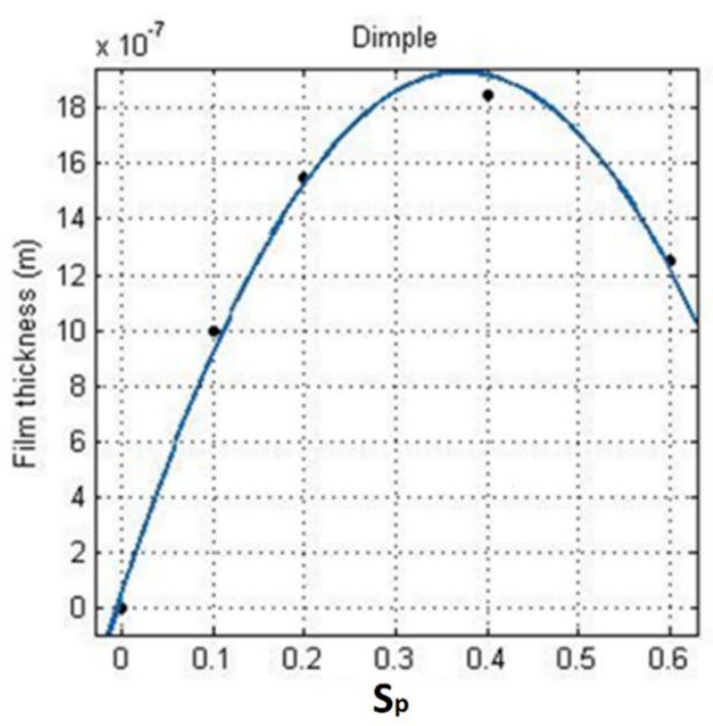

(a)

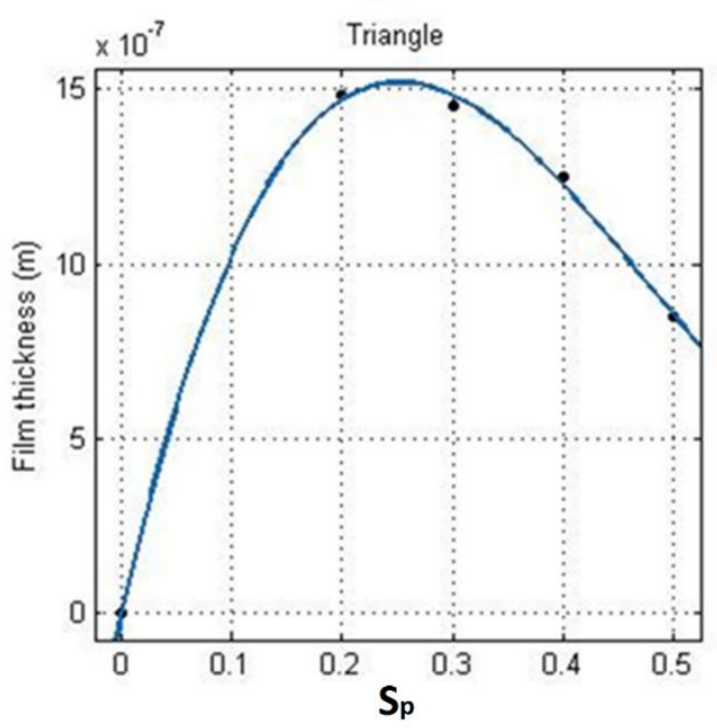

(b)

Figure 13. Film thickness curves as a function of textured area fraction $S_{p}$ for : a) Circular pocket, b) Triangular pocket.

The film thickness curves show an optimum as a function of the variable cavity geometrical ratio (Fig. 14).

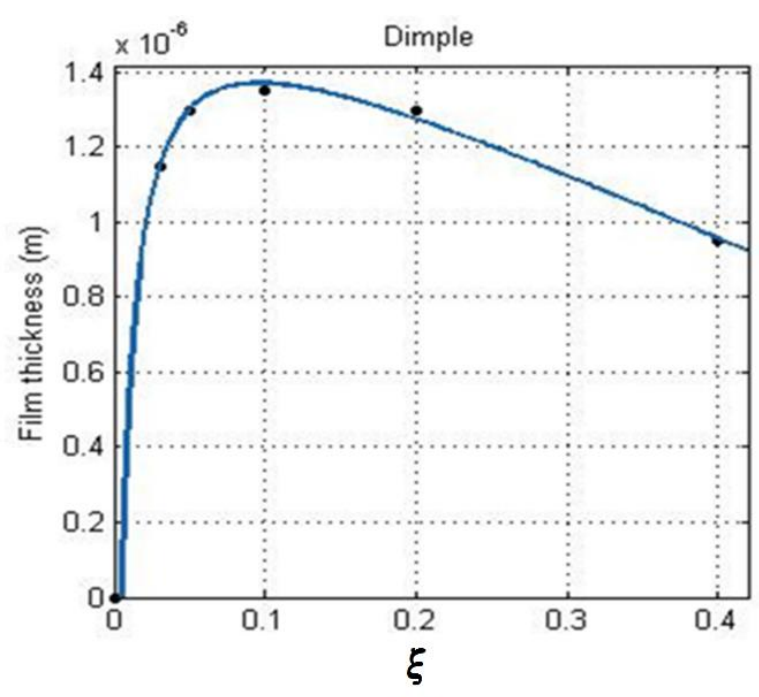

(a)

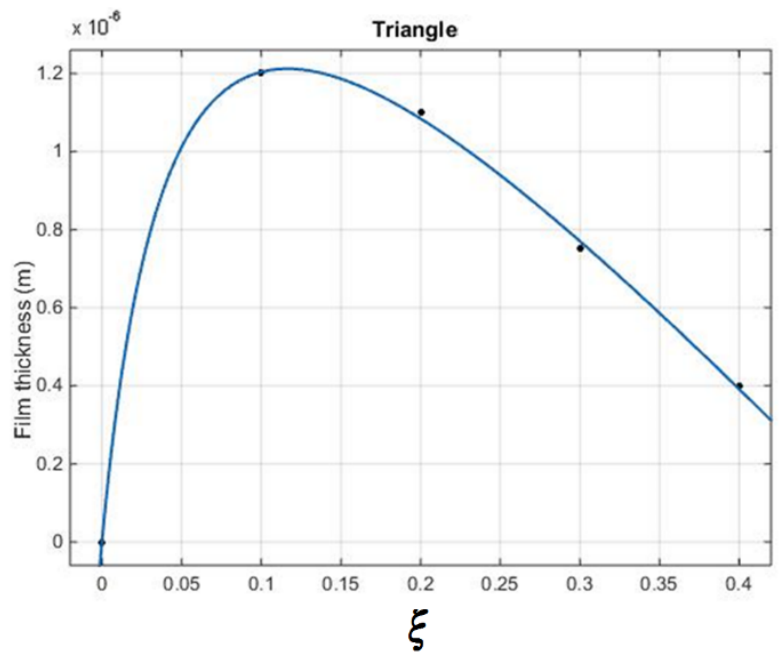

(b)

Figure 14. Film thickness curves as a function of cavity geometrical ratio $\xi$ for: $a)$ circular pocket, b) triangular pocket.

In Fig. 14, a zero cavity ratio means zero depth, which in the case of flat-flat contacts results in no pressure generation and zero film thickness. After passing the optimum value of $\xi$ the higher ratio causes the pressure drop because of the greater effect of cavitation, smaller texture size and reduction of film thickness.

Another set of simulations is performed for the chevron pattern to investigate the effect of the geometrical parameters. The simulations are based on textured area fraction and cavity geometrical ratio. These parameters can be written as (Eq. 17):

$$
\xi=\frac{T_{d}}{2 r_{p}}, \quad S_{p}=\frac{3 \sqrt{3}\left(1-K^{2}\right) r_{p}^{2}}{4 L_{g x} L_{g y}}
$$

The chevron width ratio is defined as (see Fig. 15): 


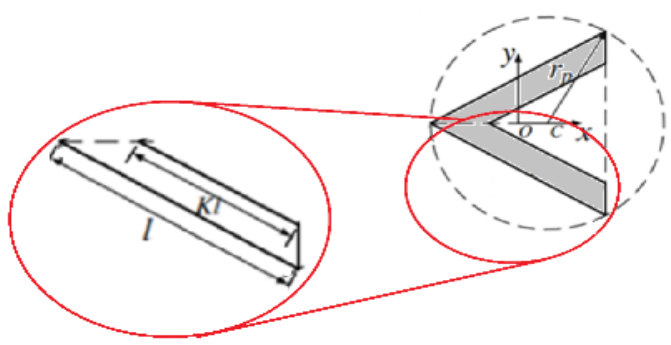

Figure 15. Definition of cavity width ratio.

Cavity width ratio: inner wall length/outer wall length = $\mathrm{K}$.

Figure 16 shows the behaviour of the film thickness based on the three aforementioned geometrical parameters. The textured area fraction $S_{p}$ and cavity geometrical ratio $\xi$ parameters have the same influence on the film thickness for a chevron pattern, as observed for the triangular and circular pockets. The third graph shows the film thickness presented as a function of the cavity width ratio $\mathrm{K}$. When $\mathrm{K}$ is equal to zero the chevron becomes a triangle, and the film thickness for the triangular pattern is smaller than for the chevron pattern. When $\mathrm{K}$ is equal to one there is no chevron pattern and the contact is in a flat-on-flat situation. Increasing $\mathrm{K}$ makes a longer wall against the flow direction in cavity and a longer wall can produce a higher pressure. This increment continues to the point at which the distance between the inlet wall of cavity and outlet wall of cavity is too short to generate more pressure. The negative effect of this short distance between the inlet and outlet walls is to reduce the film formation of the chevron pattern.

In the simulation for the geometrical ratio $\xi$ is equal to 0.1 . For the textured area fraction $S_{p}$ equal to 0.2 is applied and for both above situations the cavity width ratio $\mathrm{K}$ was constant and equal to 0.2 . The textured area operates under the average pressure $5 \mathrm{MPa}$ and the lubricant viscosity is $0.008 P a$. $s$.

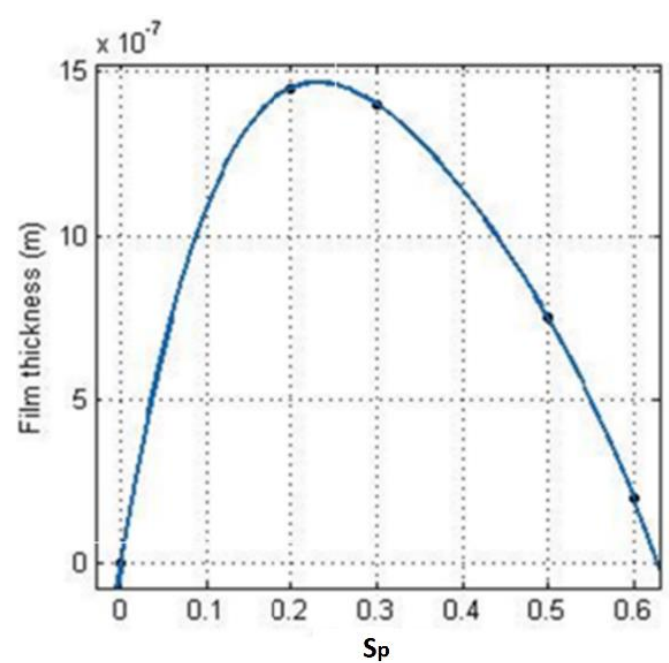

(a)

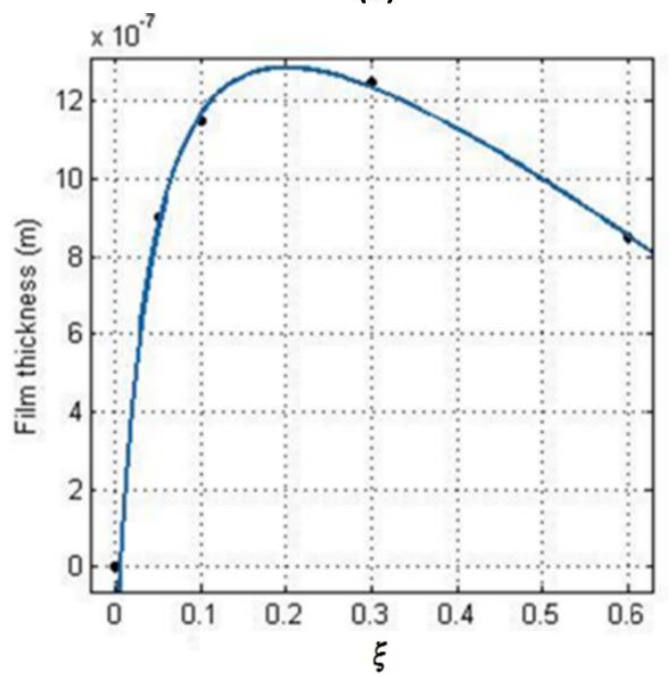

(b)

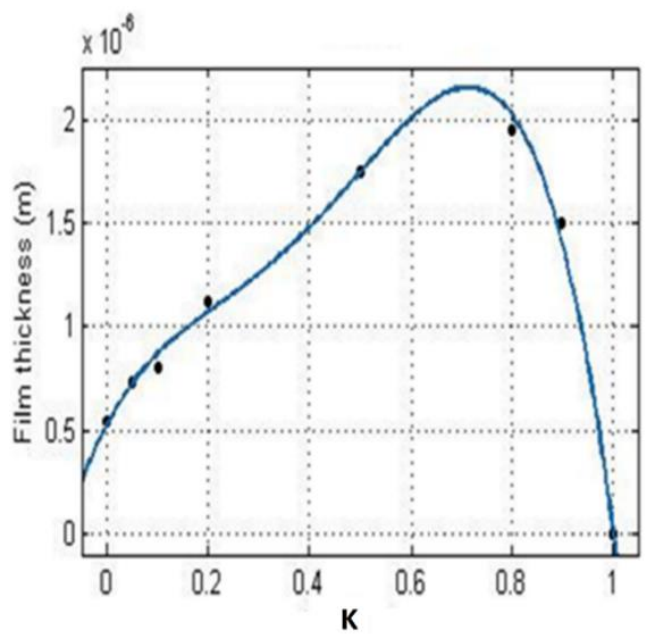

(c)

Figure 16. Film thickness curves for chevron based on : a) textured area fraction $S_{p}$, b) cavity geometrical ratio $\xi$, c) cavity width ratio $K$. 


\section{Conclusions}

The aim of the investigation was to develop a numerical code in order to have a better understanding of film thickness for textured surfaces. Simulations of textured surfaces with different patterns with different texture properties can help to modify the lubricant film thickness of contacts to the desired situation. The model is based on a numerical algorithm based on the Reynolds equation with the Elrod cavitation algorithm formulation. The equations were made discrete using the finite difference method, and solved using the TDMA iterative method. The effect of several parameters on lubricant film thickness, such as pattern type, cross-sectional shape, depth and size has been studied. The effect of lubricant leakage for the groove pattern has been studied, as well as the influence of the circular pocket arrangement.

Based on the simulation results the following conclusions are drawn:

(1) The groove pattern show the highest lubricant film formation. The reason for this beneficial effect is the higher textured area fraction over surface.

(2) The arrangement of circular pockets is not particularly relevant with regard to the lubricant film thickness.

(3) Closed groove pattern by reducing the side leakage is more effective with respect to lubricant film formation than the open groove pattern.

(4) Grooves with a rectangular cross-sectional shape are shown to be more efficient than the grooves with a circular cross-sectional shape.

(5) The optimum textured area fraction for circular pockets is calculated as being in the range of 0.3 to 0.4 .

(6) The optimum textured area fraction for triangular pockets is calculated and it is in the range of 0.2 to 0.3 .

(7) The optimum cavity geometrical ratio for circular pockets is calculated as being about 0.1 .

(8) The optimum cavity geometrical ratio for triangular pockets is calculated as being in the range of 0.11 to 0.12 .

(9) The optimum textured area fraction $S_{p}$ for chevrons is calculated as being in the range of 0.2 to 0.3 .
(10) The optimum cavity geometrical ratio $\xi$ for circular pockets is calculated as being the range of 0.2 to 0.3 .

(11) The optimum chevron width ratio $K$ is calculated as being in the range of 0.6 to 0.8 .

\section{Nomenclature}

\begin{tabular}{|c|c|c|}
\hline Parameters & Description & Unit \\
\hline$h$ & Film thickness & $m$ \\
\hline$h_{0}$ & Contact separation & $m$ \\
\hline$u_{0}$ & Sum velocity & $m \cdot s^{-1}$ \\
\hline$P$ & Dimensionless pressure & - \\
\hline$p$ & Pressure & $P a$ \\
\hline$p_{a}$ & Ambient pressure & $P a$ \\
\hline$G$ & Geometric parameter & - \\
\hline$\eta$ & Dynamic viscosity & Pa.s \\
\hline$\rho$ & Density & $\mathrm{kg} \cdot \mathrm{m}^{-3}$ \\
\hline$\rho_{c}$ & $\begin{array}{l}\text { Lubricant density in full film } \\
\text { region }\end{array}$ & $\mathrm{kg} \cdot \mathrm{m}^{-3}$ \\
\hline$F$ & $\begin{array}{l}\text { Elrod cavitation algorithm } \\
\text { switch function }\end{array}$ & - \\
\hline$\varphi$ & $\begin{array}{l}\text { Cavitation dimensionless } \\
\text { variable }\end{array}$ & - \\
\hline$T_{d}$ & Texture depth & $m$ \\
\hline$r_{p}$ & Cavity characteristic width & $m$ \\
\hline$r$ & $\begin{array}{l}\text { Texture cell length in } x \& y- \\
\text { direction, in the case of dimples }\end{array}$ & $m$ \\
\hline$L_{g x}$ & $\begin{array}{l}\text { Texture cell length in } \mathrm{x}- \\
\text { direction }\end{array}$ & $m$ \\
\hline$L_{g y}$ & $\begin{array}{l}\text { Texture cell length in } y- \\
\text { direction }\end{array}$ & $m$ \\
\hline$L_{x}$ & Textured area in $\mathrm{x}$-direction & $m$ \\
\hline$L_{y}$ & Textured area in y-direction & $m$ \\
\hline$X$ & $\begin{array}{ll}\text { Dimensionless } & \text { Cartesian } \\
\text { coordination }=x / r_{p} & \end{array}$ & - \\
\hline$Y$ & $\begin{array}{ll}\text { Dimensionless } & \text { Cartesian } \\
\text { coordination }=y / r_{p} & \end{array}$ & - \\
\hline$H$ & $\begin{array}{l}\text { Dimensionless local depth of } \\
\text { textured surface }\end{array}$ & - \\
\hline$S_{p}$ & $\begin{array}{l}\text { Textured area fraction: Cavity } \\
\text { area/Cell area, }\end{array}$ & - \\
\hline$\xi$ & $\begin{array}{l}\text { Cavity geometrical ratio: } \\
\text { Cavity depth/Cavity length. }\end{array}$ & - \\
\hline$K$ & $\begin{array}{l}\text { Cavity width ratio: inner wall } \\
\text { length/outer wall length }\end{array}$ & - \\
\hline
\end{tabular}

\section{References}

[1] Glavatskih, S. B., McCarthy, D. M. C., and Sherrington, I., 2005, "Hydrodynamic Performance of a Thrust Bearing with 
Micropatterned Pads," Tribology Transactions, 48(4), pp. 492-498.

[2] Brizmer, V., Kligerman, Y., and Etsion, I., 2003, "A Laser Surface Textured Parallel Thrust Bearing," Tribology Transactions, 46(3), pp. 397-403.

[3] Etsion, I., 2010, "State of the Art in Laser Surface Texturing," Advanced Tribology, J. Luo, Y. Meng, T. Shao, and Q. Zhao, eds., Springer Berlin Heidelberg, pp. 761-762.

[4] Etsion, I., and Burstein, L., 1996, "A Model for Mechanical Seals with Regular Microsurface Structure," Tribology Transactions, 39(3), pp. 677683.

[5] Etsion, I., Halperin, G., Brizmer, V., and Kligerman, Y., 2004, "Experimental Investigation of Laser Surface Textured Parallel Thrust Bearings," Tribology Letters, 17(2), pp. 295-300.

[6] Etsion, I., Kligerman, Y., and Halperin, G., 1999, "Analytical and Experimental Investigation of Laser-Textured Mechanical Seal Faces," Tribology Transactions, 42(3), pp. 511-516.

[7] Kovalchenko, A., Ajayi, O., Erdemir, A., Fenske, G., and Etsion, I., 2005, "The effect of laser surface texturing on transitions in lubrication regimes during unidirectional sliding contact," Tribology International, 38(3), pp. 219-225.

[8] Ronen, A., Etsion, I., and Kligerman, Y., 2001, "Friction-Reducing Surface-Texturing in Reciprocating Automotive Components," Tribology Transactions, 44(3), pp. 359-366.

[9] Ryk, G., Kligerman, Y., and Etsion, I., 2002, "Experimental Investigation of Laser Surface Texturing for Reciprocating Automotive Components," Tribology Transactions, 45(4), pp. 444-449.

[10] Vilhena, L. M., Podgornik, B., Vižintin, J., and Možina, J., 2011, "Influence of texturing parameters and contact conditions on tribological behaviour of laser textured surfaces," Meccanica, 46(3), pp. 567-575.

[11] Yu, H., Wang, X., and Zhou, F., 2010, "Geometric Shape Effects of Surface Texture on the Generation of Hydrodynamic Pressure Between Conformal Contacting Surfaces," Tribology Letters, 37(2), pp. 123-130.

[12] Ren, N., Nanbu, T., Yasuda, Y., Zhu, D., and Wang, Q., 2007, "Micro Textures in ConcentratedConformal-Contact Lubrication: Effect of Distribution Patterns," Tribol Lett, 28(3), pp. 275285.
[13] Costa, H. L., and Hutchings, I. M., 2007, "Hydrodynamic lubrication of textured steel surfaces under reciprocating sliding conditions," Tribology International, 40(8), pp. 1227-1238.

[14] Jakobsson, B., and Floberg, L., 1957, The finite journal bearing, considering vaporization: (Das Gleitlager von endlicher Breite mit Verdampfung), Chalmers University of Technology, Chalmers Tekniska Högskolas handlingar ; Avd. Maskinteknik

[15] Olsson, K. O., 1965, Cavitation in Dynamically Loaded Bearings, Scandinavian University Books, Chalmers University of Technology, Institute of Machine Elements. Report.

[16] Coyne, J. C., and Elrod, H. G., 1965, Conditions for the Rupture of a Lubrication Film.

[17] Braun, M. J., and Hannon, W. M., 2010, "Cavitation formation and modelling for fluid film bearings: A review," Proceedings of the Institution of Mechanical Engineers, Part J: Journal of Engineering Tribology, 224(9), pp. 839-863.

[18] Payvar, P., and Salant, R. F., 1992, "Computational method for cavitation in a wavy mechanical seal," Journal of Tribology, 114(1), pp. 199-204.

[19] Xiong, S., and Wang, Q. J., 2012, "Steady-state hydrodynamic lubrication modeled with the payvar-salant mass conservation model," Journal of Tribology, 134(3).

[20] Dobrica, M. B., Fillon, M., Pascovici, M. D., and Cicone, T., 2010, "Optimizing surface texture for hydrodynamic lubricated contacts using a massconserving numerical approach," Proceedings of the Institution of Mechanical Engineers, Part J: Journal of Engineering Tribology, 224(8), pp. 737750.

[21] Qiu, M., Delic, A., and Raeymaekers, B., 2012, "The effect of texture shape on the load-carrying capacity of gas-lubricated parallel slider bearings," Tribology Letters, 48(3), pp. 315-327.

[22] Qiu, M., Minson, B. R., and Raeymaekers, B., 2013, "The effect of texture shape on the friction coefficient and stiffness of gas-lubricated parallel slider bearings," Tribology International, 67, pp. 278-288.

[23] Patankar, S. V., 1980, Numerical heat transfer and fluid flow / Suhas V. Patankar, Hemisphere Pub. Corp. ; McGraw-Hill, Washington : New York.

[24] Versteeg, H. K., and Malalasekera, W., 1995, An Introduction to Computational Fluid Dynamics. The Finite Volume Method, Longman Group Ltd. 


\title{
Paper B
}

\section{The Influence of Surface Texturing on the Film Thickness in Starved Lubricated Parallel Sliding Contacts}

\author{
Dariush Bijani, Elena L. Deladi, Matthijn B. de Rooij and Dirk J. Schipper
}

Lubricants 2018

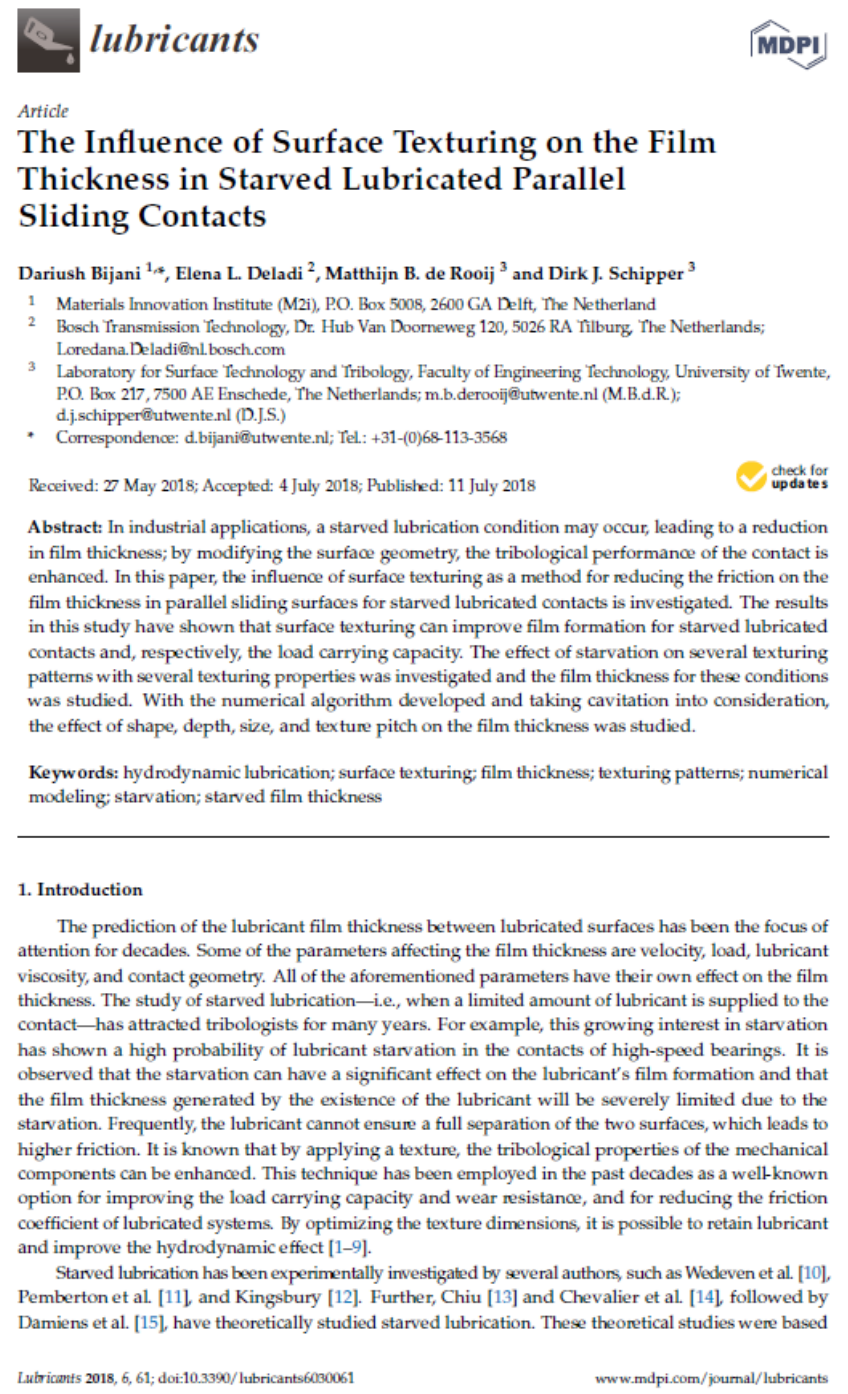





\title{
The Influence of Surface Texturing on the Film Thickness in Starved Lubricated Parallel Sliding Contacts
}

\author{
Dariush Bijani ${ }^{1, *}$, Elena L. Deladi ${ }^{2}$, Matthijn B. de Rooij ${ }^{3}$ and Dirk J. Schipper ${ }^{3}$ \\ 1 Materials Innovation Institute (M2i), P.O. Box 5008, 2600 GA Delft, the Netherland \\ 2 Bosch Transmission Technology, Dr. Hub Van Doorneweg 120, 5026 RA Tilburg, the Netherlands; \\ Loredana.Deladi@nl.bosch.com \\ 3 Laboratory for Surface Technology and Tribology, Faculty of Engineering Technology, \\ University of Twente, P.O. Box 217, 7500 AE Enschede, The Netherlands; m.b.derooij@utwente.nl (M.B.d.R.); d.j.schipper@e- \\ mail.com (D.J.S.) \\ * Correspondence: d.bijani@utwente.nl; Tel.: +31-(0)68-113-3568
}

Received: 27 May 2018; Accepted: 4 July 2018; Published: date

\begin{abstract}
In industrial applications starved lubrication conditions may occur, leading to a reduction in film thickness; by modifying the surface geometry, the tribological performance of the contact is enhanced. In this paper, the influence of surface texturing as a method for reducing the friction on the film thickness in parallel sliding surfaces for starved lubricated contacts is investigated. The results of this study have shown that surface texturing can improve film formation for starved lubricated contacts and, the load-carrying capacity. The effect of starvation on several texturing patterns with several texturing properties was investigated and the film thickness for these conditions was studied. With the numerical algorithm developed and taking cavitation into consideration, the effect of shape, depth, size, and texture pitch on the film thickness was studied.
\end{abstract}

Keywords: hydrodynamic lubrication; surface texturing; film thickness; texturing patterns; numerical modelling; starvation; starved film thickness

\section{Introduction}

The prediction of the lubricant film thickness between lubricated surfaces has been the focus of attention for decades. Some of the parameters affecting the film thickness are velocity, load, lubricant viscosity, and contact geometry. All of the aforementioned parameters have their own effect on the film thickness. The study of starved lubrication -i.e., when a limited amount of lubricant is supplied to the contact- has attracted tribologists for many years. For example, this growing interest in starvation has shown a high probability of lubricant starvation in the contacts of high-speed bearings. It is observed that the starvation can have a significant effect on the lubricant's film formation and that the film thickness generated by the existence of the lubricant will be severely limited due to the starvation. Frequently, the lubricant cannot ensure a full separation of the two surfaces, which leads to higher friction. It is known that the tribological properties of the mechanical components can be enhanced by applying a texture. This technique has been employed in the past decades as a well-known option for improving the load-carrying capacity and wear resistance, and for reducing the friction coefficient of lubricated systems. By optimizing the texture dimensions it is possible to retain lubricant and improve the hydrodynamic effect [1-9].

Starved lubrication has been investigated experimentally by several authors, such as Wedeven et al. [10], Pemberton et al. [11], and Kingsbury [12]. Further, Chiu [13] and Chevalier et al. [14], followed by Damiens et al. [15], have theoretically studied starved lubrication. These theoretical studies were based on the work of Jakobsson and Floberg [16] and Olsson [17], who introduced the concept of "fractional film content" and derived continuity relations.

The influence of starvation on the lubrication of rigid cylinders was studied by Floberg [18]. Dalmaz and Godet [19] studied the influence of inlet starvation on the reduction in film thickness in the case of a sphere against a lubricated plate. In the work of Brewe and Hamrock [20], the influence of starvation was studied theoretically. In this work, by using the Reynolds boundary condition and by systematically reducing the 
fluid inlet level, they observed the pressure build-up for a given film thickness. In their study, the inlet meniscus boundary was taken as the start of the pressure buildup. They analyzed a wide range of geometry parameters from a ball on a plate to a ball in a conforming groove. Moreover, for a fully flooded conjunction, the film thickness formula was modified to incorporate the starvation effect. Boness [21] showed experimentally the importance of the starvation effects on the bearing failure and wear. Chevalier et al. [22] used a numerical hydrodynamic analysis. They studied the effect of repeated passes on the film thickness with a constant and harmonic oil inlet. In their study, they employed the work of Elrod et al. [23,24] and showed that the shape of the oil inlet film could influence film thickness behaviour. Cann and Lubrecht [25] further studied the properties of the lubricant and four variable parameters- oil volume, velocity, load, and viscosity and the influence of each parameter on lubricant film thickness. In their work, they presented the film thickness for a wide range of velocities and different lubricant parameters.

For fully flooded (elasto-) hydrodynamic lubrication, micro-geometric cavities have been successfully used to improve the lubrication between surfaces. These micro-scale cavities can increase the film thickness and the load-carrying capacity, can entrap wear debris, and can act as micro-reservoirs; see for instance Etsion et al. [2,5,6]. Surface texturing also influences the transitions between the lubrication regimes [7], i.e., the lubrication regime transformation between full film, mixed, and boundary lubrication.

In the work of Ryk et al. [9], the possible advantages of applying laser surface texturing (LST) to piston rings have been demonstrated theoretically and experimentally. By employing the full LST rings, the optimum texturing parameters, in the case of the minimum friction force, were found. Their work found good agreement with experimental results. Their results revealed the benefits of surface texturing in full lubrication conditions, as well as starved lubrication conditions. Based on this study, in an internal combustion engine application, the employment of micro-grooves in cylinder liners show lower fuel consumption and wear. The textured surfaces provide a thicker lubricant film than the non-textured surfaces in which the grooves act as local Rayleigh step bearings. Experimental measurements showed that, due to texturing, the textured bearing can generate a lubricant film about three times thicker than the film thickness of the non-textured bearing throughout the range of the tested loads. In this paper, the effect of texturing (meso-geometry), including cavitation, on the lubricant film characteristics was studied for macroscopically starved lubricated plane-parallel sliding surfaces. The surfaces were smooth, i.e. no roughness (microgeometry).

\section{Materials and Methods}

In 1886, Osborne Reynolds derived a partial differential equation to calculate the pressure build-up in self-acting bearings. This equation has been used successfully to explain the fluid film pressure distribution in lubricated contacts. This equation can be deduced from the Navier-Stokes equations under the narrow gap assumption that the Reynolds number of the fluid flow in the gap between the surfaces is small. The Reynolds equation in Cartesian coordinates can be written as Equation (1):

$$
\begin{aligned}
\frac{\partial}{\partial x}\left(\frac{\rho h^{3}}{\eta}\right. & \left.\frac{\partial p}{\partial x}\right)+\frac{\partial}{\partial y}\left(\frac{\rho h^{3}}{\eta} \frac{\partial p}{\partial y}\right) \\
& =6\left(u_{0}\right) \frac{\partial(\rho h)}{\partial x} \\
& +6 \rho h \frac{\partial\left(u_{0}\right)}{\partial x}+12 \frac{\partial(\rho h)}{\partial t}
\end{aligned}
$$

In this equation, $h$ is the film thickness, $\eta$ is the viscosity, $u_{0}$ is the sum velocity, and $\rho$ is the density of the lubricant. In sliding direction at the outlet of the cavity, the lubricant is dragged through a converging region and, as a result, pressure is generated. At the opening of the cavity, the flow diverges; according to the Reynolds equation (Equation (1)), this results in a negative pressure. However, a negative pressure is suppressed by the lubricant.

In the case of lubricated systems under a moderate load, the Jakobsson-Floberg-Olsson (JFO) $[16,17]$ cavitation theory is used. It is not suitable for cases where surface tension plays an important role, such as in face seals [26]. Based on the JakobssonFloberg-Olsson theory, in lubricated contacts when cavitation is present, there are two zones of lubrication. In the first zone the lubricant film is complete, and the pressure varies in this region, therefore the Reynolds equation is applicable. In the second zone, due to the cavitation existence, only a fraction of the lubricant film gap is occupied by the lubricant film. The pressure within the cavitation area is taken as constant [26].

According to the Payvar-Salant $[27,28]$ model and taking cavitation into account, the steady-state massconservation Reynolds equation in a Cartesian coordinate system can be written as Equation (2) [29]:

$$
\begin{aligned}
& \frac{\partial}{\partial x}\left(\frac{h^{3}}{\eta} \frac{\partial(F \varphi)}{\partial x}\right)+\frac{\partial}{\partial y}\left(\frac{h^{3}}{\eta} \frac{\partial(F \varphi)}{\partial y}\right) \\
& =\frac{6 u_{0}}{p_{a}-p_{c}} \frac{\partial((1+(1-F) \varphi) h)}{\partial x} \\
& \text { In the above equation, } p_{a} \text { is the ambient pressure, } \\
& p_{c} \text { is the cavitation pressure, } F \text { is a dimensionless }
\end{aligned}
$$


dependent variable, and $\varphi$ is the cavitation index. For more information, see Xiong and Wang [29].

Dobrica et al. [30] carried out an investigation based on the influence of cavitation and different texturing parameters on the hydrodynamic performance of textured surfaces. Their numerical study was based on the Reynolds equation with the JFO formulation on the plane-parallel contact and the sliding inclined contact. Their calculations focused on finding the optimum dimensions of the textured region and dimple aspect ratio. In their investigation on the plane-parallel textured sliding contacts, due to the cavitation a significant influence on the performance is observed. The existence of cavitation in partially textured parallel sliders has a positive effect due to the increase of inlet flow (inlet suction). Furthermore, in convergent sliders with a high incline ratio, texturing shows a minimal effect, as well as cavitation. In this study, for different plane-inclined sliders, the optimal dimple depth and length were determined.

\section{Mathematical Solution}

Four different texture patterns have been investigated: circular pocket, triangular pocket, chevron, and groove. Figure 1 shows the different cavity shapes and the parameters characterizing their geometries. The chevron pattern is defined by two similar equilateral triangles of different sizes. The triangular pocket is a special case of the chevron with the inner edge length of the chevron equal to zero. For these two cases, the centre of the unit cell coincides with the midpoint of the altitude line of the triangle or chevron shape; see also [31]. All patterns investigated in this study had a rectangular cross-sectional profile. The general film gap can be formulated as Equation (3):

$$
h=h_{0}+h_{\text {macro }}+h_{\text {texture }}
$$

In the case of flat-flat contact, the macro geometry is omitted and Equation (3) reduces to Equation (4) [31,32]:

$$
\frac{h(x, y)}{h_{0}(x, y)}=1+H(x, y)
$$

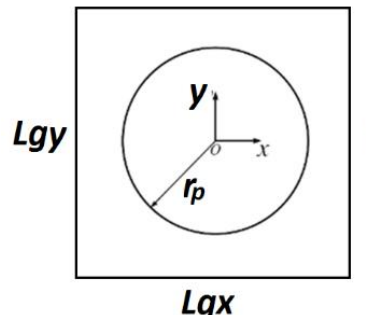

(a)

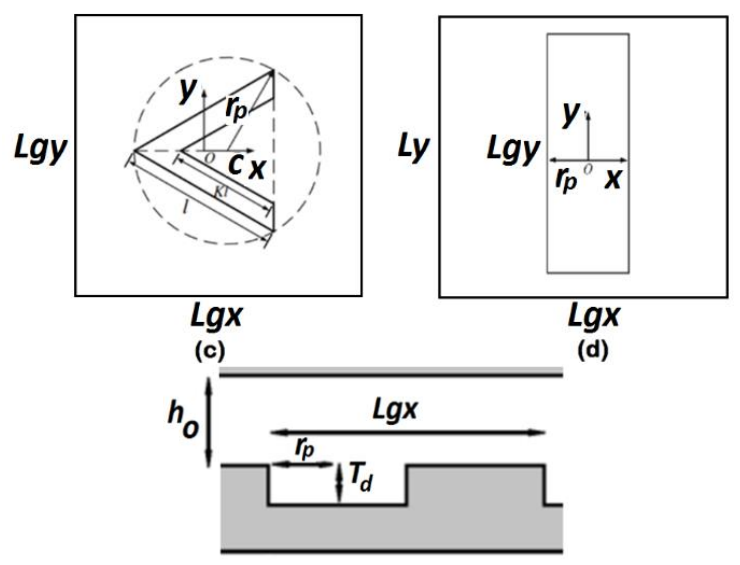

(e)
Figure 1. Geometrical scheme of patterns, (a) circular pocket; (b) triangular pocket; (c) chevron; and (d) groove; (e) schematic illustration of cavity profile (Reproduced with permission from Mingfeng Qiu, Bret R. Minson,

Bart Raeymaekers, Tribology International, published by Elsevier, 2013) [31].

The formula for the circular pocket is given in Equation (5):

$$
H(x, y)=\left\{\begin{array}{cc}
0, & X^{2}+Y^{2}>1 \\
\frac{T_{d}}{h_{0}}, & X^{2}+Y^{2} \leq 1
\end{array}\right.
$$

where $X=\frac{x}{r_{P}}$ and $Y=\frac{y}{r_{P}}$. The film thickness formula for the triangular pocket can be written as Equation (6):

$$
\begin{gathered}
H(x, y)=\left\{\begin{array}{c}
0,(X, Y) \notin \Omega \\
\frac{T_{d}}{h_{0}},(X, Y) \in \Omega
\end{array}\right. \\
\Omega:-\frac{3}{4} \leq X \\
\leq \frac{3}{4} \text { and }\left\{\begin{array}{c}
\frac{1}{\sqrt{3}} X-\frac{\sqrt{3}}{4} \leq Y \leq \frac{1}{\sqrt{3}} X+\frac{\sqrt{3}}{4} \\
-\frac{1}{\sqrt{3}} X-\frac{\sqrt{3}}{4} \leq Y \leq-\frac{1}{\sqrt{3}} X+\frac{\sqrt{3}}{4}
\end{array}\right.
\end{gathered}
$$

The formula for the chevron can be written as Equation (7):

$$
\begin{aligned}
& H(x, y)=\left\{\begin{array}{cc}
0, & (X, Y) \notin \Omega \\
\frac{T_{d}}{h_{0}}, & (X, Y) \in \Omega
\end{array}\right. \\
& H(x, y)=\left\{\begin{aligned}
0, & (X, Y) \notin \Omega \\
\frac{T_{d}}{h_{0}}, & (X, Y) \in \Omega
\end{aligned}\right.
\end{aligned}
$$




$$
\leq \frac{3}{4} \text { and }\left\{\begin{array}{c}
\Omega:-\frac{3}{4} \leq X \\
\frac{1}{\sqrt{3}} X+\frac{\sqrt{3}}{2}\left(K-\frac{1}{2}\right) \leq \\
Y \leq \frac{1}{\sqrt{3}} X+\frac{\sqrt{3}}{4} \\
-\frac{1}{\sqrt{3}} X-\frac{\sqrt{3}}{4} \leq \\
Y \leq-\frac{1}{\sqrt{3}} X+\frac{\sqrt{3}}{2}\left(\frac{1}{2}-K\right)
\end{array}\right.
$$

The formula for the grooves is given in Equation (8):

$$
\begin{gathered}
H(x, y)=\left\{\begin{array}{lr}
0, & (X, Y) \notin \Omega \\
\frac{T_{d}}{h_{0}}, & (X, Y) \in \Omega
\end{array}\right. \\
\Omega:-\frac{1}{2} \leq X \leq \frac{1}{2} \text { and } \frac{1}{2} \leq Y \leq \frac{1}{2}
\end{gathered}
$$

The tri-diagonal matrix algorithm (TDMA) is used to solve Equation (2). Furthermore, to reduce the storage needed for calculation, the line-by-line TDMA solver (Patankar [33]) is applied. In the case of a twodimensional problem, the TDMA becomes iterative, and sweeping is done line-by-line and column-bycolumn or row-by-row [34].

After developing the numerical code, the next step is to check the validity of the code. In this study, to validate the model and algorithm, a comparison was made between the experimental measurements from the literature and the calculated results of this algorithm. In this validation, the experimental results were provided from the work of Kovalchenko et al. [35]. In their work, they investigated the effect of size and the density of dimples on the coefficient of friction. In Figure 2, two types of dimple arrays from this work are presented.

\section{Disk 3}

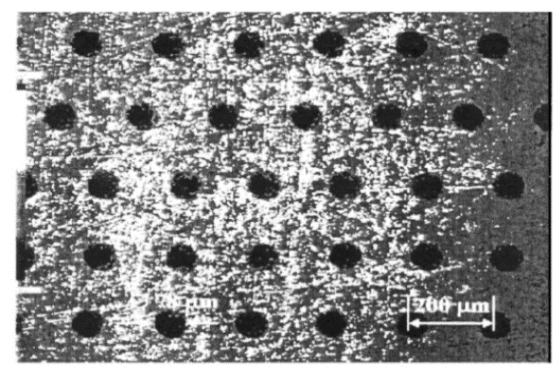

(a)
Disk 6

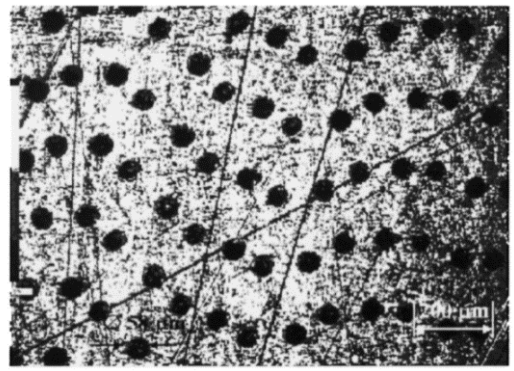

(b)

Figure 2. (a) Disk 3 dimple array, (b) Disk 6 dimple array (Reproduced with permission from Andriy Kovalchenko, Oyelayo Ajayi, Ali Erdemir, et al., Tribology Transactions, published by Taylor and Francis, 2004) [40].

The geometrical properties of these two samples are presented in Table 1.

The measurement results for these two disks are presented in Figure 3.

From Figure 3 it is possible to extract the experimental data. Further, by using the numerical model for the full film non-starved condition when the lubricant kinematic viscosity is $1247 \mathrm{cSt}$ at $40^{\circ} \mathrm{C}$, and by applying $20 \mathrm{~N}$ as the normal load, the hydrodynamic lubrication friction is calculated. The simulated texture array is presented in Figure 4.

Table 1. Geometrical properties of the textured samples (Reproduced with permission from Andriy Kovalchenko, Oyelayo Ajayi, Ali Erdemir et al., Tribology Transactions, published by Taylor and Francis, 2004) [35].

$\begin{array}{ccc} & \text { Disk 3 } & \text { Disk 6 } \\ \text { Depth of dimples } & 5.5 \mu \mathrm{m} & 5 \mu \mathrm{m} \\ \text { Diameter of dimples } & 78 \mu \mathrm{m} & 58 \mu \mathrm{m} \\ \text { Distance between dimples } & 200 \mu \mathrm{m} & 100 \mu \mathrm{m}\end{array}$

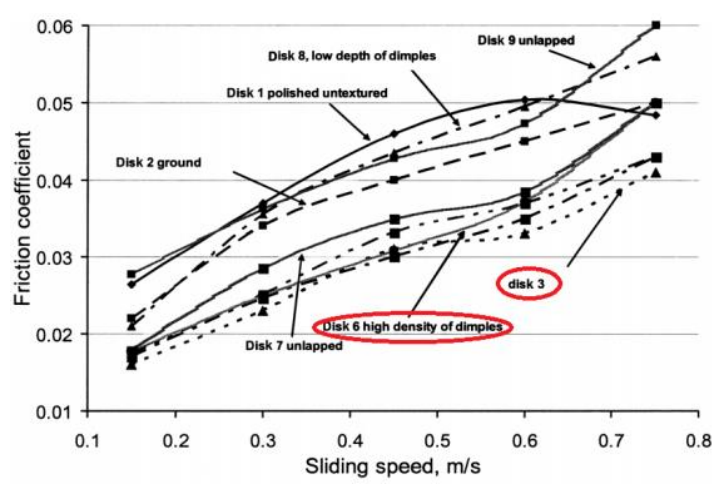

Figure 3. Measurement results (Reproduced with permission from Andriy Kovalchenko, Oyelayo Ajayi, Ali Erdemir, et al., Tribology Transactions, published by Taylor and Francis, 2004) [35]. 


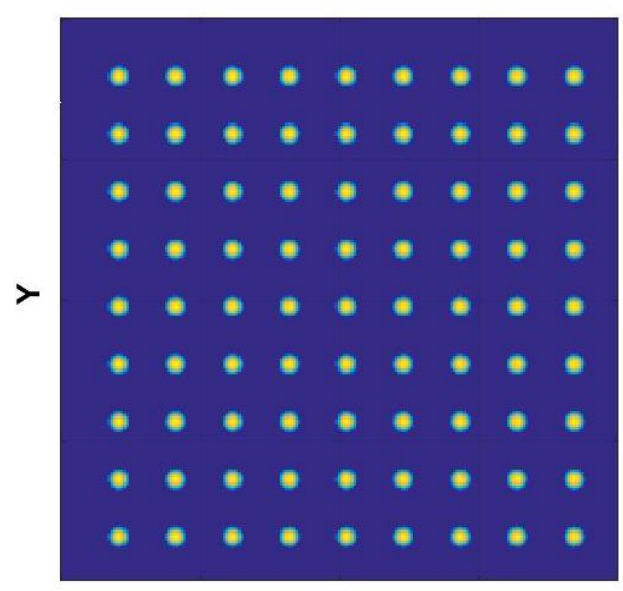

$\mathbf{x}$

(a)

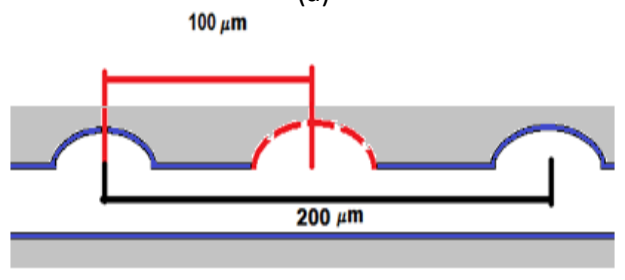

(b)

Figure 4. (a) Simulated texture array and (b) cavity profile.

The comparison between the numerical results and experimental measurements for the abovementioned dimple types is presented in Figure 5.

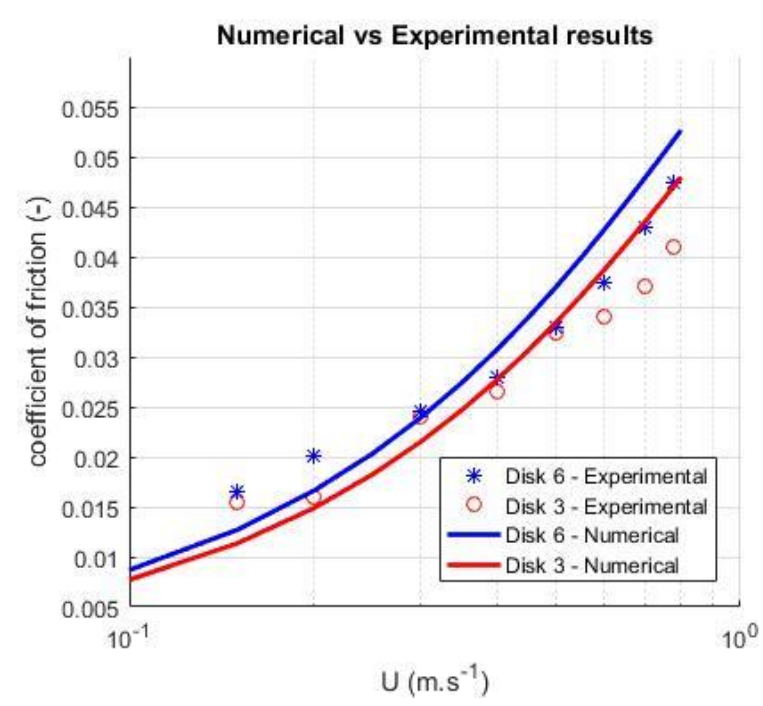

Figure 5. Comparison between numerical and experimental results.

From Figure 5, it is possible to see that there is a difference between the values of coefficient of friction calculated by the numerical model and the experimentally measured results of this parameter. For example, when the velocity is $0.5 \mathrm{~m} \mathrm{~s}^{-1}$, the calculated value is around $9 \%$ higher than the measured value for disk 6 and less than 1\% higher for disk 3. This figure shows that the calculated coefficient of friction has almost the same trend and value as the measured coefficient of friction.

In this study, after the validation of the algorithm, several simulations were carried out to investigate the influence of starvation on textured surfaces with various texturing patterns and to investigate the film thickness behaviour of the film thickness based on the different texturing parameters. The number of pockets and distance between them are defined by introducing a new parameter, pitch. The pitch for the chevron, triangle, and circular pockets was calculated as follows:

Texture size $=S=2 \times r_{p}$

Pitch in $\mathrm{x}$ direction: $P_{i}=\frac{S}{L_{g x}}$; see Figure 1 for a definition of the geometry.

\section{Results}

Several simulations were performed in order to understand the effect of limited lubricant supply on the calculated film thickness for different texturing parameters for different patterns.

The film thickness obtained by texturing influenced by the limited amount of lubricant in the input region of the contact. By considering different values for the lubricant supply thickness $\left(h_{\text {oil }}\right)$, the effect of this parameter over the starved film thickness was calculated. Furthermore, the effect of texturing parameters -i.e., pattern type, texture pitch $\left(P_{i}\right)$, texture depth $\left(T_{d}\right)$, and texture size $(S)$ - on the starved film thickness $\left(h_{s}\right)$ was studied. These simulations were based on surfaces with grooved, circular, triangular, and chevron pockets, presented in Figure 6.

To study the effect of the value of lubricant supply thickness $\left(h_{\text {oil }}\right.$ ) over different texturing patterns, $h_{\text {oil }}$ was assumed to equal $2.5 \mu \mathrm{m}$. In Figure 7, a schematic illustration of starved lubricated contact is presented. 


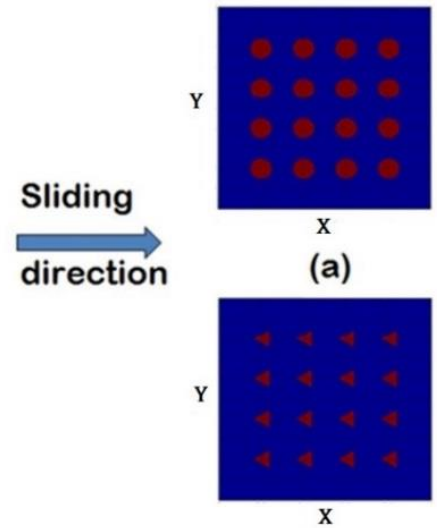

(c)

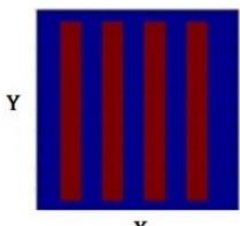

(b)

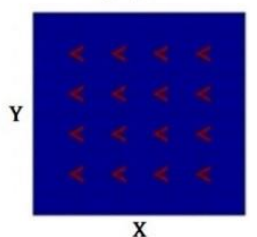

(d)
Figure 6. Schematic illustration of different patterns.

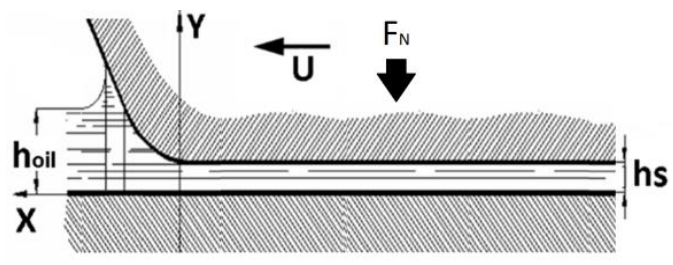

Figure 7. Schematic illustration of limited lubricant supply $\left(h_{\text {oil }}\right)$ and calculated film thickness $\left(h_{s}\right)$.

When the texture pitch $\left(P_{i}\right)$, texture size $(S)$, and texture depth $\left(T_{d}\right)$ values were zero or close to zero, there was no film formation because of the absence of the wedge effect in flat-flat contact and the lack of texturing; therefore, there was no pressure generation. By increasing the values of $T_{d}, P_{i}$ and $S$ textures appeared over the surface and pressure generation occurred in the contact due to the wedge effect. By increasing the texture depth and after passing the optimum value of this parameter a higher depth resulted in a pressure drop due to the higher effect of cavitation. If the pitch value tends to a maximum value of itself -which is equal to one- then $S=L_{g x}$. That means that there was no texture on the surface of a flat-flat contact to build pressure at the contact. In the case of cavity size, after $S$ passed a specific value, the bigger size reduced the number of cavities until a single cavity existed over the surface. The existence of only one cavity would mean that there was only one outlet cavity wall against the lubricant flow. Therefore, larger cavity sizes can reduce the effect of texturing.

\subsection{Grooves}

The film thickness obtained with a patterned, grooved surface with different values for $T_{d}$ and $S$ and $P_{i}$ is shown in Figure 8.

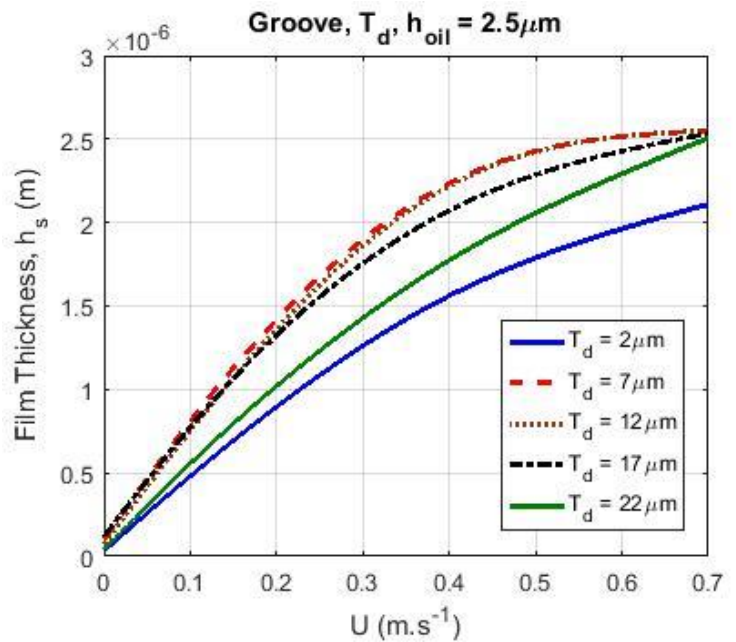

(a)

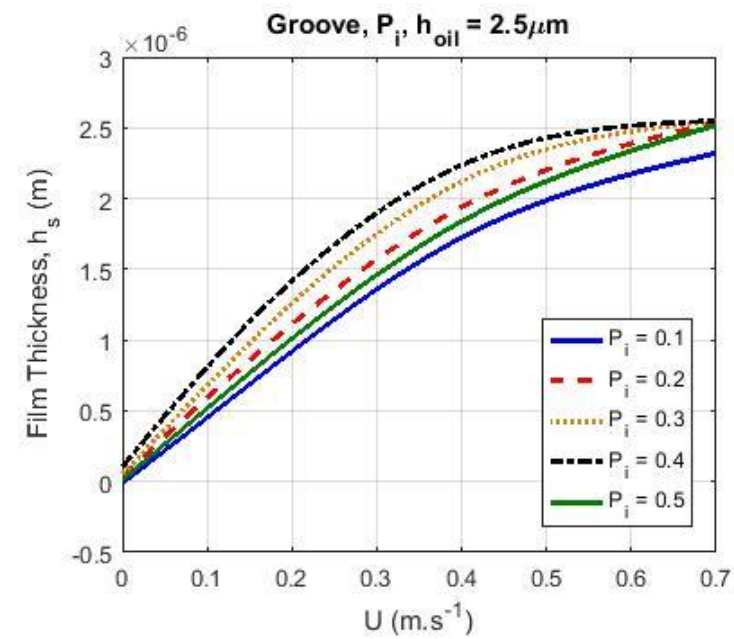

(b)

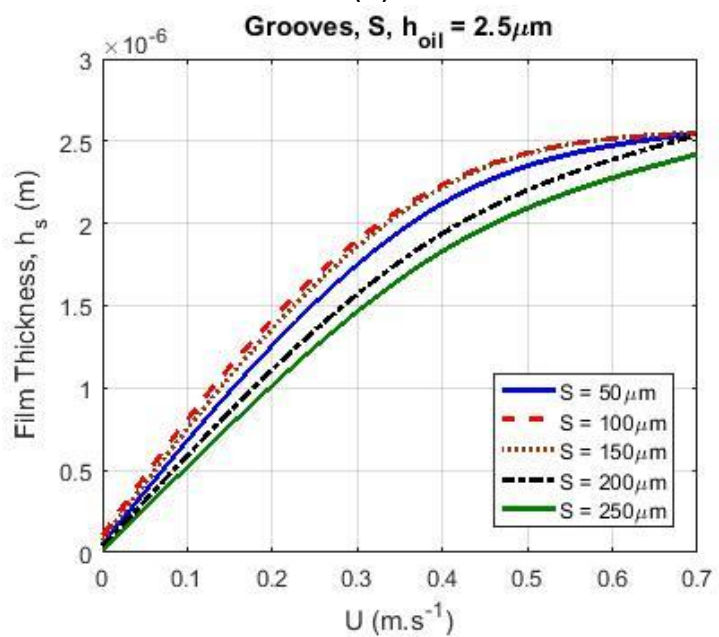

(c)

Figure 8. Film thickness as a function of velocity obtained by grooves for $h_{\text {oil }}=2.5 \mu \mathrm{m}$, for different values of (a) $T_{d}$, (b) $P_{i}$, and (c) $S$.

Figure 8a shows the effect of a limited lubricant supply on starved film thickness for different values of the texture depth parameter. When the sliding was in 
the lower velocity range $\left[0 \mathrm{~m} . \mathrm{s}^{-1}-0.3 \mathrm{~m} . \mathrm{s}^{-1}\right]$, the film thickness was more sensitive to increasing texture depth than when the sliding velocity was in the higher velocity range $\left[0.3 \mathrm{~m} . \mathrm{s}^{-1}-1-0.7 \mathrm{~m} . \mathrm{s}^{-1}\right]$. In Figure $8 \mathrm{~b}$, when the contacts were sliding in the lower range of the velocity, the film thickness increased when $P_{i}$ was increased from 0.1 to 0.5 . However, after passing $P_{i}=0.4$, there was a decrease in film thickness. The aforementioned effect of the variation of $P_{i}$ on the film thickness was sensitive when the surface sliding velocity was low. However, for higher velocities, the starvation effect had a greater influence on the film thickness. For high velocities, the film thickness was less sensitive to the pitch.

The influence of texture size $(S)$ on the starved film thickness is shown in Figure 8c. From this calculation it is shown that, by increasing the velocity, the effect of employing the optimum values for texture size on film thickness was on the decrease and textures with different sizes had a tendency towards the same values of film thickness.

Figure 9 shows the film thickness for grooves. The effect of the lubricant supply $h_{\text {oil }}$ on $h_{s}$ was studied for the geometry resulting in the highest film thickness. In this calculation, $h_{\text {oil }}$ varied from $0.5 \mu \mathrm{m}$ to $3.5 \mu \mathrm{m}$, and $\mathrm{T}_{\mathrm{d}}=7 \mu \mathrm{m}, \mathrm{S}=100 \mu \mathrm{m}$, and $P_{i}=0.4$ are shown.

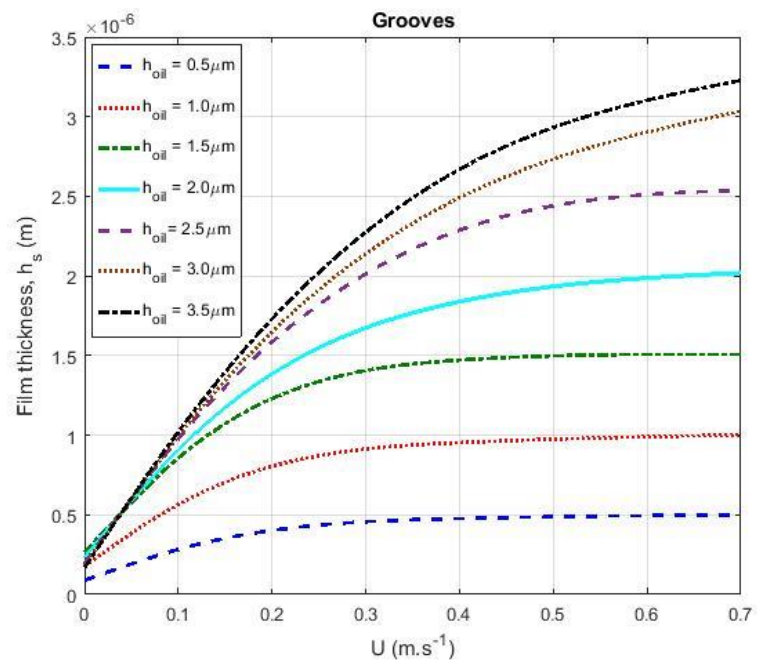

Figure 9. Effect of $h_{\text {oil }}$ on $h_{s}$.

In this paper, to compare the effect of the lubricant supply $h_{\text {oil }}$ on different patterns, the same geometry that had been applied in Figure $9\left(T_{d}=\right.$ $7 \mu \mathrm{m}, \mathrm{S}=100 \mu \mathrm{m}$ and $P_{i}=0.4$ ) was utilized. In [36], the maximum film thickness was obtained for different patterns when the values for the texturing parameters were in the same range as those used in the calculations for Figure $9\left(T_{d}=7 \mu \mathrm{m}, \mathrm{S}=100 \mu \mathrm{m}\right.$, and $\left.P_{i}=0.4\right)$.

\subsection{Dimples}

The effect of a fixed lubricant supply $h_{\text {oil }}=2.0 \mu \mathrm{m}$ on $h s$ for a circular pocket pattern was also studied. It is worth mentioning that by employing the circular pockets and the same operating conditions used for the other patterns, the maximum film thickness generated was around $2.5 \mu \mathrm{m}$. Therefore, if the applied lubricant supply was similar to the other patterns $\left(h_{\text {oil }}=\right.$ $2.5 \mu \mathrm{m})$, it would not be possible to observe a sensible effect of starvation on the film thickness behaviour. To better understand the effect of starvation on film thickness for circular pockets, less lubricant supply was applied $\left(h_{\text {oil }}=2.0 \mu \mathrm{m}\right)$. The calculated film thickness for circular pockets (when $T_{d}=7 \mu \mathrm{m}, S=100 \mu \mathrm{m}$ and $P_{i}=0.4$ ) is shown in Figure 10 .

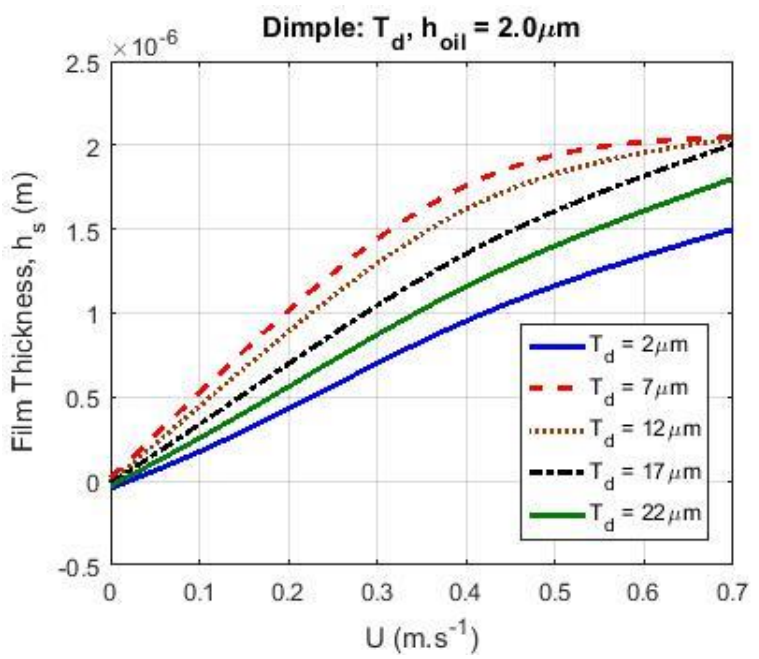

(a)

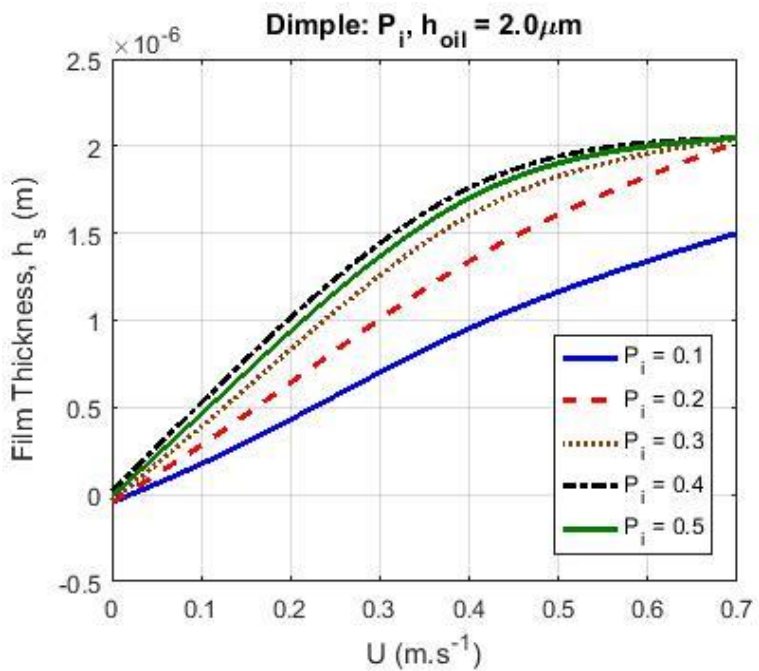

(b) 


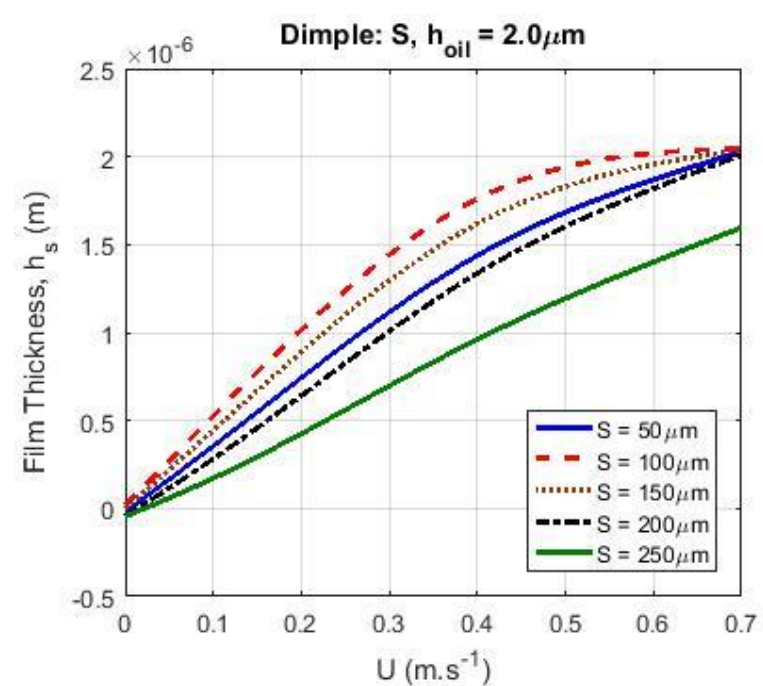

(c)

Figure 10. Film thickness as a function of the sliding velocity for circular pockets when $h_{\text {oil }}=$ $2.0 \mu \mathrm{m}$, for different values of (a) $T_{d}$, (b) $P_{i}$, and (c) $S$.

It was shown that the film thickness increased with the sliding velocity in the lower velocity range by increasing the texturing parameters $\left(P_{i}, T_{d}\right.$ and $\left.S\right)$ toward the optimum value. This effect of variation for different texturing properties on the film thickness was sensible when the surface sliding velocity was in the lower range of velocities [0-0.3 $\mathrm{m} \mathrm{s}^{-1}$ ] (Figure 10). However, at higher velocities $\left[0.3 \mathrm{~m} . \mathrm{s}^{-1}-0.7 \mathrm{~m} . \mathrm{s}^{-1}\right]$, the starvation effect exerted a greater influence on the film thickness. When the texturing parameter values were not too far from the optimum value of those parameters, at high velocities, the film thickness was less sensitive to the texture properties.

\subsection{Triangular Pockets}

The influence of different texturing parameters on starved film thickness for triangular pockets was investigated. The starved film thickness is presented in Figure 11; the calculations were based on $h_{\text {oil }}=$ $2.5 \mu \mathrm{m}$.

For triangular pockets, as shown in Figure 11, the effect of variation in texturing parameters on the film thickness was similar to the calculations that were presented for the circular pockets. When the sliding velocity was low, by increasing the optimum value of the texturing parameters $\left(P_{i}, T_{d}\right.$ and $\left.S\right)$, the film thickness also increased. This effect of the variation of different texturing properties on film thickness was smaller when the surface sliding velocity was higher.

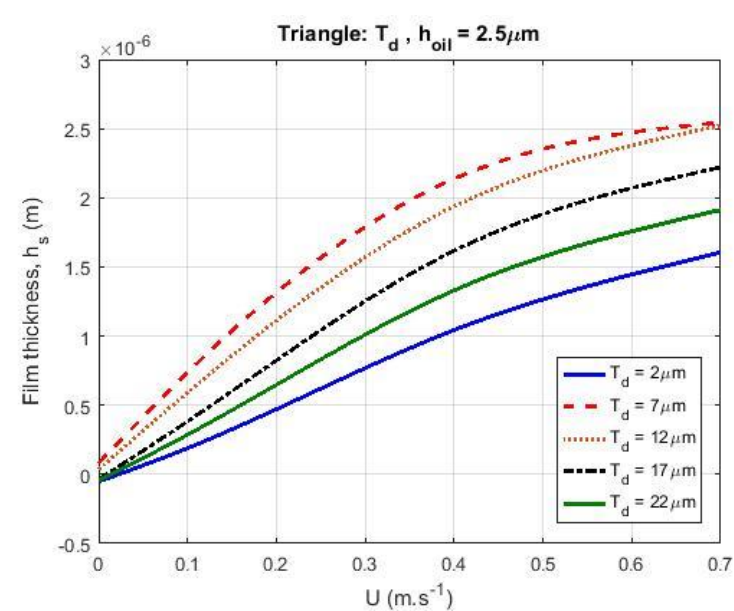

(a)

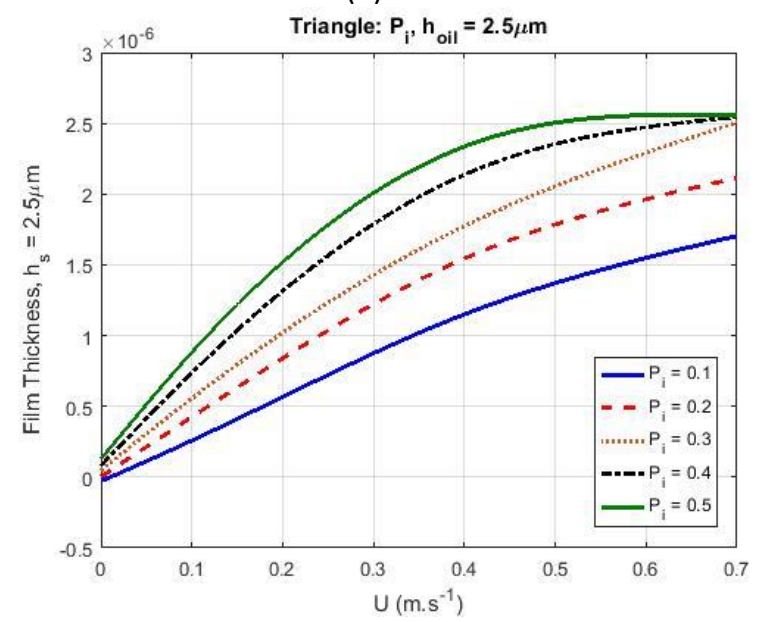

(b)

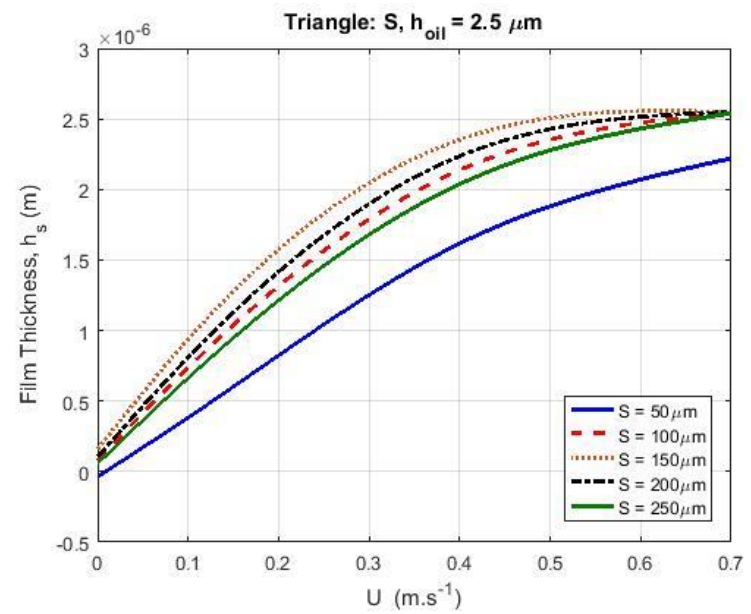

(c)

Figure 11. Film thickness as a function of the sliding velocity for triangular pockets when $h_{\text {oil }}=2.5 \mu \mathrm{m}$ for different values of (a) $T_{d}$, (b) $P_{i}$ and (c) $S$.

\subsection{Chevrons}

The effect of different texturing parameters on the starved film thickness in the case of chevronshaped pocket patterns is presented in Figure 12, and 
these calculations were based on a lubricant supply $h_{\text {oil }}=2.5 \mu \mathrm{m}$.

In the case of chevron patterns (see Figure 12), the effect of variation of texturing parameters was similar to the previous calculations. The only difference was in the optimum values for the texturing properties.

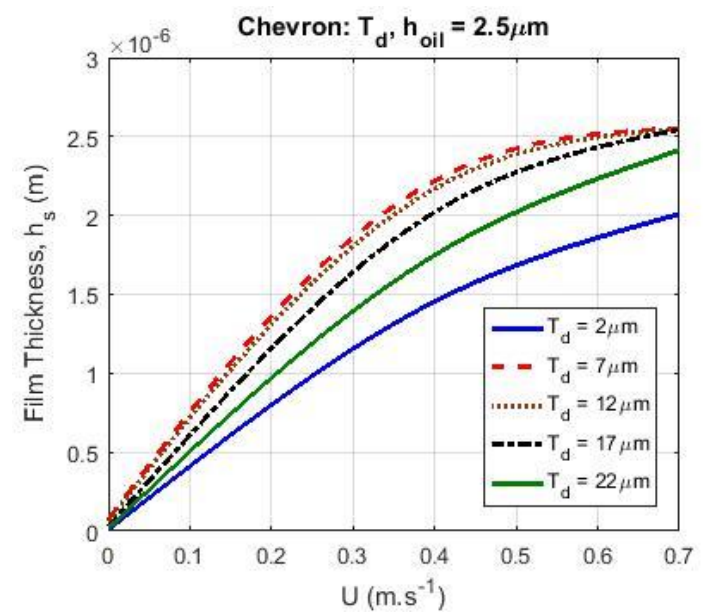

(a)

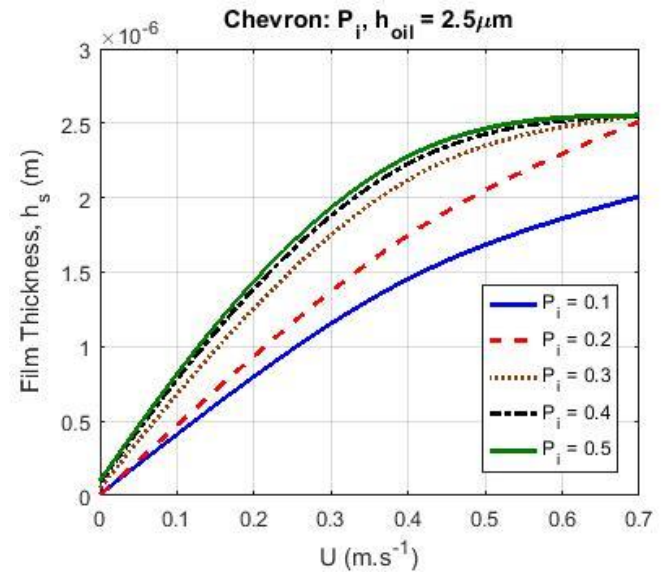

(b)

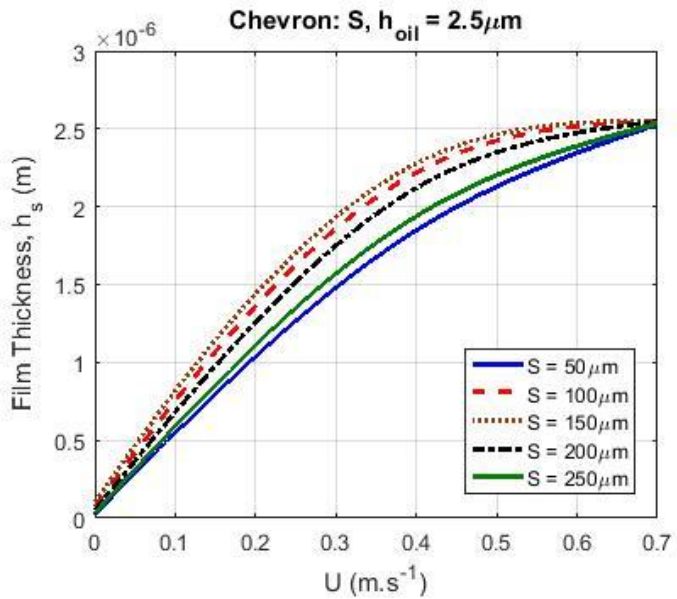

(c)

Figure 12. Film thickness as a function of sliding velocity for chevron-shaped pockets when $h_{\text {oil }}=$ $2.5 \mu \mathrm{m}$ for different values of (a) $T_{d}$, (b) $P_{i}$ and (c) $S$.

\subsection{Comparison of Surface Patterns}

In Figure 13, film thickness as a function of velocity is shown for different patterns when $T_{d}=7 \mu \mathrm{m}, S=$ $100 \mu \mathrm{m}$ and $P_{i}=0.4$. In these calculations, the effect of different texture patterns as a function of hs was studied: $\quad h_{\text {oil }}=2.0 \mu \mathrm{m} ; h_{\text {oil }}=2.5 \mu \mathrm{m} ; h_{\text {oil }}=3.0 \mu \mathrm{m}$ and $3.5 \mu \mathrm{m}$.

For different levels of lubricant supply $\left(h_{\text {oil }}=\right.$ $2.0 \mu \mathrm{m} ; h_{\text {oil }}=2.5 \mu \mathrm{m} ; h_{\text {oil }}=3.0 \mu \mathrm{m} ; h_{\text {oil }}=$ $3.5 \mu \mathrm{m}$ ), the groove pattern had a promising effect for generating the greatest film thickness for sliding velocities between $0 \mathrm{~m} . \mathrm{s}^{-1}$ and $0.3 \mathrm{~m} . \mathrm{s}^{-1}$. The chevron pattern produced a thicker film than the triangular and circular pockets. Moreover, by employing the circular pocket pattern the lowest film thickness was obtained. In Figure $13 a$, when $h_{\text {oil }}$ was equal to $2 \mu \mathrm{m}$ the effect of starvation on the calculated film thickness was greater. In this case, at higher velocities the film thickness generated by different patterns had the same value.

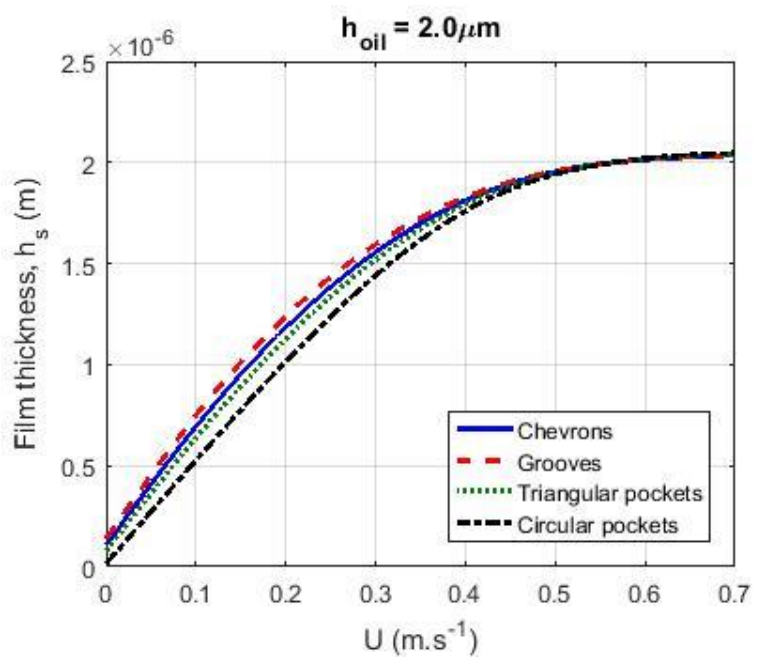

(a)

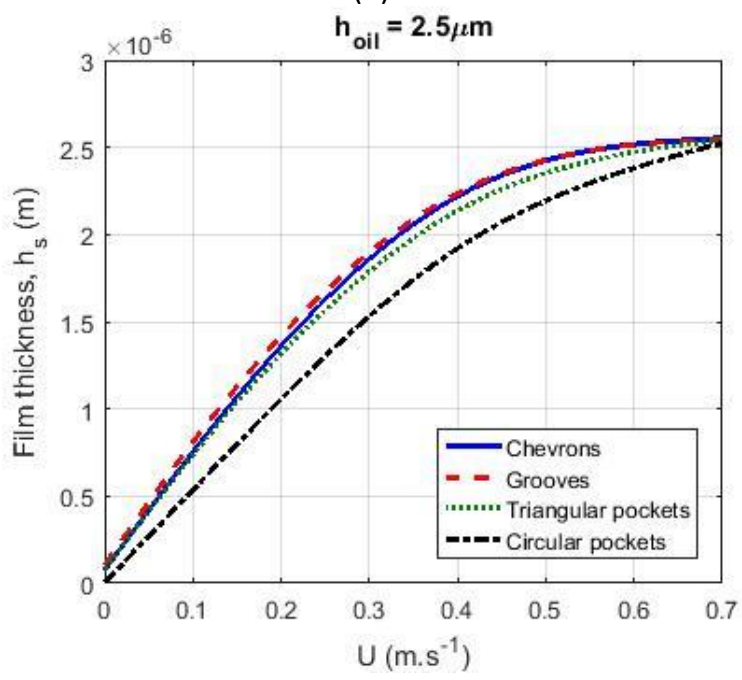


(b)

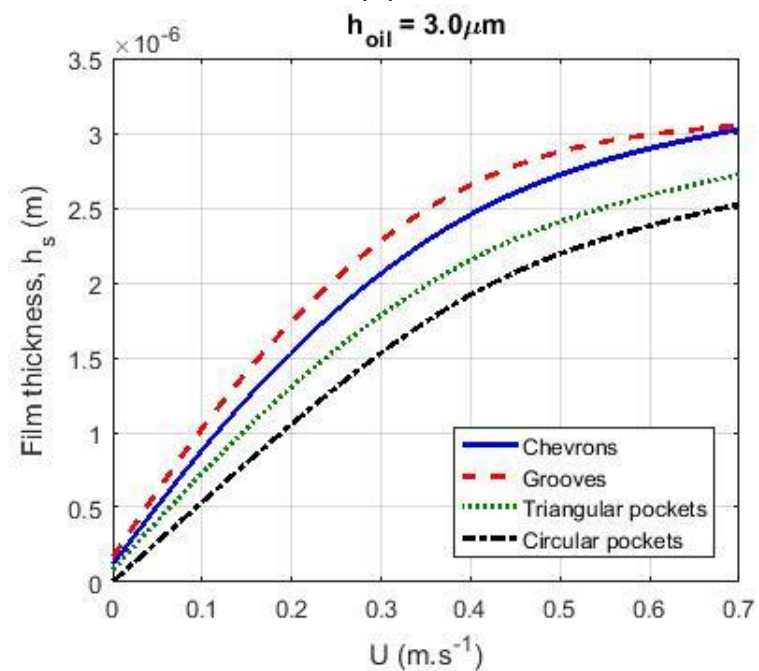

(c)

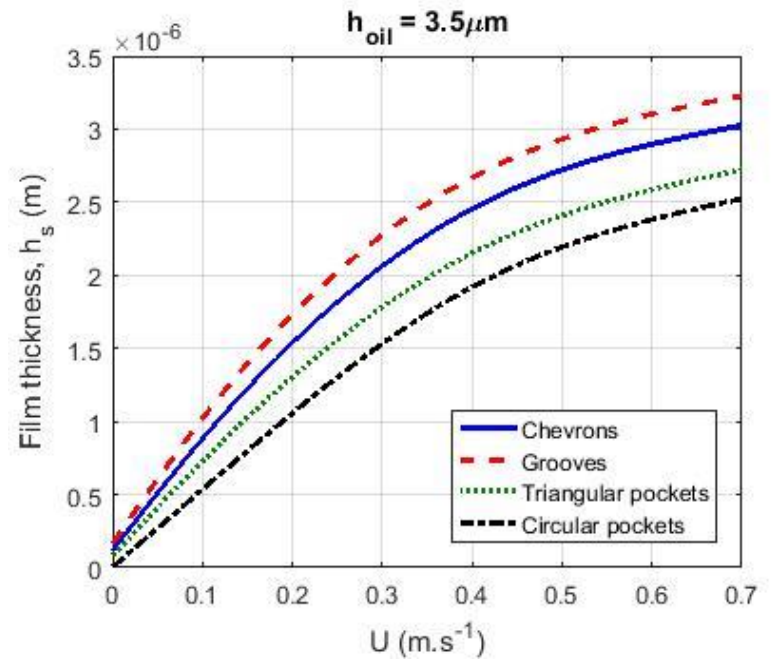

(d)

Figure 13. Effect of limited lubricant supply with different texturing patterns for (a) $h_{\text {oil }}=2.0 \mu \mathrm{m}$,

(b) $h_{\text {oil }}=2.5 \mu \mathrm{m}$ (c) $h_{\text {oil }}=3.0 \mu \mathrm{m}$, and

(d) $h_{\text {oil }}=3.5 \mu \mathrm{m}$.

By increasing $h_{\text {oil }}$ the effect of starvation on the film thickness decreased. For instance, when $h_{\text {oil }}=$ $3.5 \mu \mathrm{m}$ (see Figure 13d) the variation in film thickness based on different texturing patterns was achievable at high and low velocities, unlike for $h_{\text {oil }}=2.0 \mu \mathrm{m}$ (Figure 13a) where at higher velocities there was no difference between the film thickness generated by the different patterns. In this case, the groove pattern had the most promising effect for gaining a higher film thickness.

When the distance between the texture cells were the same (pitch is constant) the groove pattern was more successful in generating load-carrying capacity because of the higher textured area fraction per unit area. Chevrons were more efficient than triangular pockets and circular pockets because of the existence of a longer outlet wall in the cavity zone.
In summary, the results in Figure 13 show that the greatest film thickness was achieved for the grooves and the smallest film thickness was found when circular pockets were applied. Compared to the triangular pockets, the chevrons were more efficient.

\section{Conclusions}

A numerical code has been developed to predict the lubricant film formation on textured surfaces for starved lubricated contacts. Simulations of textured surfaces with different patterns and different texture properties showed that the lubricant film thickness of such contacts could be adjusted to the desired situation with texture properties. The lubrication model was based on a numerical algorithm using the Reynolds equation with the Elrod cavitation algorithm formulation. The equations were made discrete using the finite difference method and solved using the TDMA iterative method. The effect of several parameters on the starved film thickness, such as pattern type, depth, size, and texture pitch, has been studied.

- Of the patterns analyzed, the groove pattern showed the highest lubricant film formation due to the higher textured area fraction of the surface.

- In the case of starved lubrication, increasing the dimensions of the texture parameters $\left(P_{i}, T_{d}\right.$ and $S$ ) resulteds in increased film thickness at low velocities. After passing the optimum value of the texture parameters for different patterns, there was a decrease in film thickness. The aforementioned effect on film thickness was visible when the surface sliding velocity was low. At high velocities when the influence of geometry and the optimization of texturing parameters are more sensible, the starvation effect exerted a greater influence on the film thickness. It is worth mentioning that this sensitivity to the texturing parameters depended on the pattern type and operational conditions for different cases. Further, at velocities higher than $0.6 \mathrm{~m} . \mathrm{s}^{-1}$, the film thickness was less sensitive to texture properties.

- For different lubricant supply values $h_{\text {oil }}$, the groove pattern showed the highest film thickness at low velocities. The chevron pattern generated a larger film thickness than the triangular and circular pockets and the smallest film thickness was found for the circular pocket pattern.

- For small values of $h_{\text {oil }}=2 \mu \mathrm{m}$, the effect of starvation on calculated film thickness was greater. For high velocities, the generated film 
thickness in this case for the different patterns was the same.

- By increasing the lubricant supply $\left(h_{\text {oil }}\right)$, the texturing pattern geometry had a larger influence on the generated film thickness, while starvation reduced the effect of the texture on the film thickness.

Author Contributions: "Conceptualization, D.J. Schipper, E.L. Deladi and D. Bijani; Methodology, D.J. Schipper and D. Bijani; Software, D. Bijani.; Validation, D. Bijani and D.J. Schipper; Formal Analysis, D. Bijani and D.J. Schipper; Investigation, D. Bijani and D.J. Schipper; Writing-Original Draft Preparation, D. Bijani; Writing-Review \& Editing, D. Bijani, E.L. Deladi, M.B. de Rooij and D.J. Schipper; Supervision, D.J. Schipper.

Funding: This research was funded by Materials Innovation Institute (M2i) grant number M21.1.11448.

Acknowledgments: The authors would like to gratefully acknowledge Dr. Nilgoon Irani for her help on writing this article.

Conflicts of Interest: The authors declare no conflict of interest.

\section{Nomenclature}

\begin{tabular}{|c|c|c|}
\hline Parameters & Description & Unit \\
\hline$h$ & film thickness & $\mathrm{m}$ \\
\hline$h_{0}$ & contact separation & $\mathrm{m}$ \\
\hline$u_{0}$ & sum velocity & $\mathrm{m} \cdot \mathrm{s}^{-1}$ \\
\hline$P$ & dimensionless pressure & - \\
\hline$p$ & pressure & $\mathrm{Pa}$ \\
\hline$p_{a}$ & ambient pressure & $\mathrm{Pa}$ \\
\hline$G$ & geometric parameter & - \\
\hline$\eta$ & dynamic viscosity & Pa s \\
\hline$\rho$ & density & $\mathrm{kg} \cdot \mathrm{m}^{-3}$ \\
\hline$\rho_{c}$ & $\begin{array}{l}\text { lubricant density in full film } \\
\text { region }\end{array}$ & $\mathrm{kg} \cdot \mathrm{m}^{-3}$ \\
\hline$F$ & $\begin{array}{l}\text { Elrod cavitation algorithm } \\
\text { switch function }\end{array}$ & - \\
\hline$\varphi$ & $\begin{array}{l}\text { cavitation dimensionless } \\
\text { variable }\end{array}$ & - \\
\hline$T_{d}$ & texture depth & $\mathrm{m}$ \\
\hline$r_{p}$ & cavity characteristic width & $\mathrm{m}$ \\
\hline$r$ & $\begin{array}{l}\text { texture cell length in } x \& y \text { - } \\
\text { direction, in the case of dimples }\end{array}$ & $\mathrm{m}$ \\
\hline$L_{g x}$ & $\begin{array}{l}\text { texture cell length in the } x \text { - } \\
\text { direction }\end{array}$ & $\mathrm{m}$ \\
\hline$L_{g y}$ & $\begin{array}{l}\text { texture cell length in the } y \text { - } \\
\text { direction }\end{array}$ & $\mathrm{m}$ \\
\hline$L_{x}$ & textured area in the $x$-direction & $\mathrm{m}$ \\
\hline$L_{y}$ & textured area in the $y$-direction & $\mathrm{m}$ \\
\hline
\end{tabular}

$\begin{array}{llll}X & \begin{array}{l}\text { dimensionless } \\ \text { 7coordination }=\frac{x}{r_{p}}\end{array} & \text { Cartesian } & \text { - } \\ Y & \begin{array}{l}\text { dimensionless } \\ \text { coordination }=\frac{y}{r_{p}}\end{array} & \text { Cartesian } & - \\ & & & \\ H & \text { dimensionless local depth of } & - \\ & \text { textured surface } & & \\ \text { textured cavity size }=2 \times r_{p} & \end{array}$

\section{References}

[1] Glavatskih, S. B., McCarthy, D. M. C., and Sherrington, I., 2005, "Hydrodynamic Performance of a Thrust Bearing with Micropatterned Pads," Tribology Transactions, 48(4), pp. 492-498.

[2] Brizmer, V., Kligerman, Y., and Etsion, I., 2003, "A Laser Surface Textured Parallel Thrust Bearing," Tribology Transactions, 46(3), pp. 397-403.

[3] Etsion, I., 2010, "State of the Art in Laser Surface Texturing," Advanced Tribology, J. Luo, Y. Meng, T. Shao, and Q. Zhao, eds., Springer Berlin Heidelberg, pp. 761-762.

[4] Etsion, I., and Burstein, L., 1996, "A Model for Mechanical Seals with Regular Microsurface Structure," Tribology Transactions, 39(3), pp. 677683.

[5] Etsion, I., Halperin, G., Brizmer, V., and Kligerman, Y., 2004, "Experimental Investigation of Laser Surface Textured Parallel Thrust Bearings," Tribology Letters, 17(2), pp. 295-300.

[6] Etsion, I., Kligerman, Y., and Halperin, G., 1999, "Analytical and Experimental Investigation of Laser-Textured Mechanical Seal Faces," Tribology Transactions, 42(3), pp. 511-516.

[7] Kovalchenko, A., Ajayi, O., Erdemir, A., Fenske, G., and Etsion, I., 2005, "The effect of laser surface texturing on transitions in lubrication regimes during unidirectional sliding contact," Tribology International, 38(3), pp. 219-225.

[8] Ronen, A., Etsion, I., and Kligerman, Y., 2001, "Friction-Reducing Surface-Texturing in Reciprocating Automotive Components," Tribology Transactions, 44(3), pp. 359-366.

[9] Ryk, G., Kligerman, Y., and Etsion, I., 2002, "Experimental Investigation of Laser Surface Texturing for Reciprocating Automotive Components," Tribology Transactions, 45(4), pp. 444-449.

[10] Wedeven, L. D., Evans, D., and Cameron, A., 1971, "Optical analysis of ball bearing starvation," J Lubric Technol Trans ASME, 93 Ser F(3), pp. 349363.

[11] Pemberton, J., and Cameron, A., 1976, "A mechanism of fluid replenishment in 
elastohydrodynamic contacts," Wear, 37(1), pp. 185-190.

[12] Kingsbury, E., 1973, "Cross flow in a starved EHD contact," ASLE Transactions, 16(4), pp. 276-280.

[13] Chiu, Y. P., 1974, "An analysis and prediction of lubricant film starvation in rolling contact systems," ASLE Transactions, 17(1), pp. 22-35.

[14] Chevalier, F., Lubrecht, A. A., Cann, P. M. E., Colin, F., and Dalmaz, G., 1998, "Film thickness in starved EHL point contacts," Journal of Tribology, 120(1), pp. 126-132.

[15] Damiens, B., Venner, C. H., Cann, P. M. E., and Lubrecht, A. A., 2004, "Starved lubrication of elliptical EHD contacts," Journal of Tribology, 126(1), pp. 105-111.

[16] Jakobsson, B., and Floberg, L., 1957, The finite journal bearing, considering vaporization: (Das Gleitlager von endlicher Breite mit Verdampfung), Chalmers University of Technology, Chalmers Tekniska Högskolas handlingar ; Avd. Maskinteknik

[17] Olsson, K. O., 1965, Cavitation in Dynamically Loaded Bearings, Scandinavian University Books, Chalmers University of Technology, Institute of Machine Elements. Report.

[18] Floberg, L., 1961, "Boundary conditions of cavitation regions in journal bearings," ASLE Transactions, 4(2), pp. 282-286.

[19] Dalmaz, G., and Godet, M., 1973, "Traction, load and film thickness in lightly-loaded lubruicated point contacts," Journal Mechanical Engineering Science, 15(6).

[20] Brewe, D., and Hamrock, B., 1982, "Analysis of starvation effects on hydrodynamic lubrication in nonconforming contacts," Trans. ASME, Journal Lubrication Technology, 104, pp. 410-417.

[21] Boness, R. J., 1970, "Effect of oil supply on cage and roller motion in a lubricated roller bearing.," J Lubric Technol Trans ASME, 92 Ser F(1), pp. 39-53.

[22] Chevalier, F., Lubrecht, A. A., Cann, P. M. E., Colin, F., and Dalmaz, G., 1995, "Starved film thickness: a qualitative explanation," Tribology Series, 30, pp. 249-257.

[23] Elrod, H. G., 1981, "A Cavitation algorithm," Journal of lubrication technology, 103(3), pp. 350354.

[24] Elrod, H. G., and Adams, M. L., 1975, "A computer program for cavitation and starvation problems.."

[25] Cann, P. M. E., and Lubrecht, A. A., 2007, "Bearing performance limits with grease lubrication: The interaction of bearing design, operating conditions and grease properties," Journal of Physics D: Applied Physics, 40(18), pp. 5446-5451.
[26] Coyne, J. C., and Elrod, J. H. G., 1970, "Conditions for the Rupture of a Lubricating Film. Part I: Theoretical Model," Journal of Lubrication Technology, 92(3), pp. 451-456.

[27] Payvar, P., and Salant, R. F., 1992, "Computational method for cavitation in a wavy mechanical seal," Journal of Tribology, 114(1), pp. 199-204.

[28] Braun, M. J., and Hannon, W. M., 2010, "Cavitation formation and modelling for fluid film bearings: A review," Proceedings of the Institution of Mechanical Engineers, Part J: Journal of Engineering Tribology, 224(9), pp. 839-863.

[29] Xiong, S., and Wang, Q. J., 2012, "Steady-state hydrodynamic lubrication modeled with the payvar-salant mass conservation model," Journal of Tribology, 134(3).

[30] Dobrica, M. B., Fillon, M., Pascovici, M. D., and Cicone, T., 2010, "Optimizing surface texture for hydrodynamic lubricated contacts using a massconserving numerical approach," Proceedings of the Institution of Mechanical Engineers, Part J: Journal of Engineering Tribology, 224(8), pp. 737 750.

[31] Qiu, M., Minson, B. R., and Raeymaekers, B., 2013, "The effect of texture shape on the friction coefficient and stiffness of gas-lubricated parallel slider bearings," Tribology International, 67, pp. 278-288.

[32] Qiu, M., Delic, A., and Raeymaekers, B., 2012, "The effect of texture shape on the load-carrying capacity of gas-lubricated parallel slider bearings," Tribology Letters, 48(3), pp. 315-327.

[33] Patankar, S. V., 1980, Numerical heat transfer and fluid flow / Suhas V. Patankar, Hemisphere Pub. Corp. ; McGraw-Hill, Washington : New York.

[34] Versteeg, H. K., and Malalasekera, W., 1995, An Introduction to Computational Fluid Dynamics. The Finite Volume Method, Longman Group Ltd.

[35] Kovalchenko, A., Ajayi, O., Erdemir, A., Fenske, G., and Etsion, I., 2004, "The effect of laser texturing of steel surfaces and speed-load parameters on the transition of lubrication regime from boundary to hydrodynamic," Tribology Transactions, 47(2), pp. 299-307.

[36] Bijani, D., Deladi, L. E., and Schipper, D. J., 2016, "The Influence of Surface Texturing on the Film Thickness in Parallel Sliding Surfaces," 20th International Colloquium Tribology: Technische Akademie Esslingen, p. 12. 


\title{
Paper C
}

\section{The Influence of Surface Texturing on the Frictional Behaviour of Parallel Sliding Lubricated Surfaces under Conditions of Mixed Lubrication}

\author{
Dariush Bijani, Elena L. Deladi, Aydar Akchurin, Matthijn B. de Rooij and Dirk J. Schipper
}

Lubricants 2018

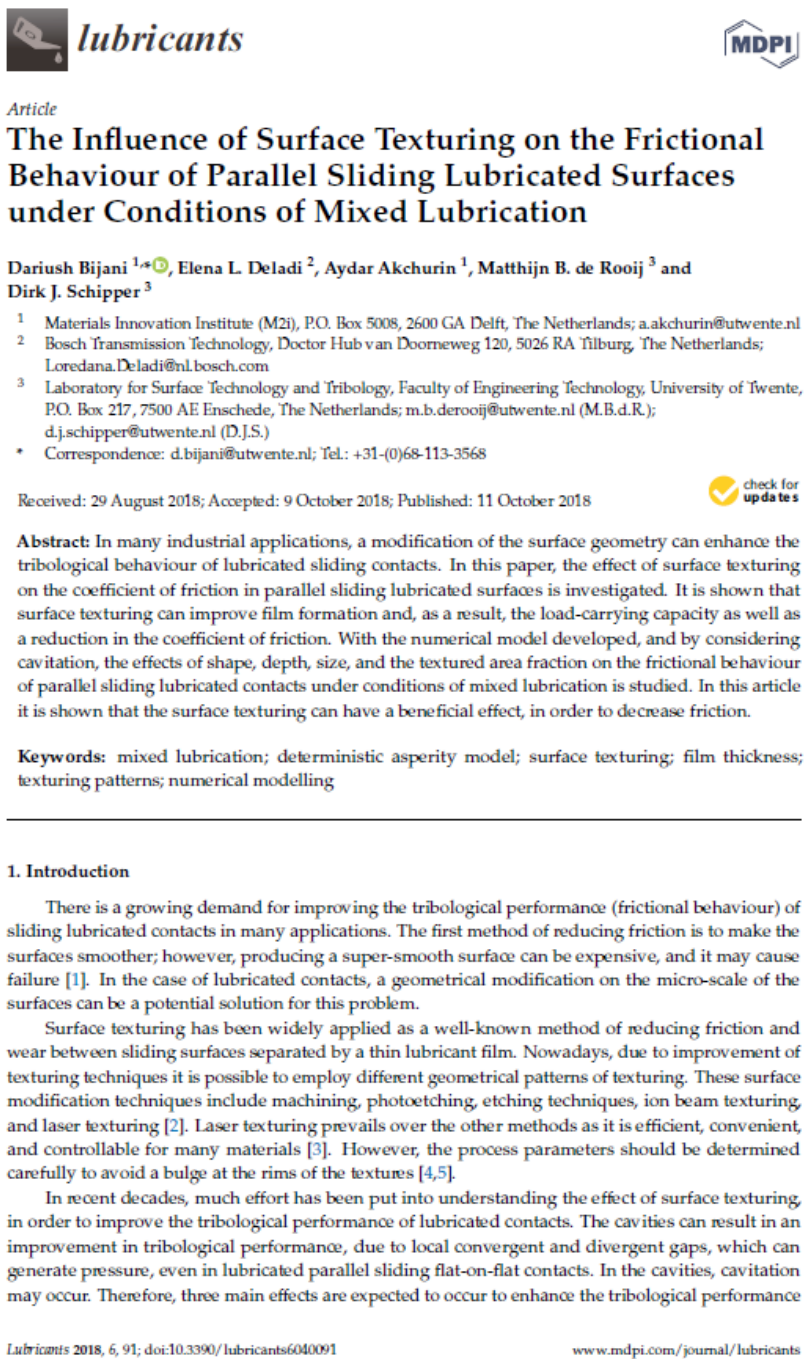





\title{
The Influence of Surface Texturing on the Frictional Behaviour of Parallel Sliding Lubricated Surfaces under Conditions of Mixed Lubrication
}

\author{
Dariush Bijani ${ }^{1, *}$, Elena L. Deladi ${ }^{2}$, Aydar Akchurin ${ }^{1}$, Matthijn B. de Rooij ${ }^{3}$ and Dirk J. Schipper ${ }^{3}$ \\ 1 Materials Innovation Institute (M2i), P.O. Box 5008, 2600 GA Delft, The Netherlands; a.akchurin@utwente.nl \\ 2 Bosch Transmission Technology, Doctor Hub van Doorneweg 120, 5026 RA Tilburg, The Netherlands; \\ Loredana.Deladi@nl.bosch.com \\ 3 Laboratory for Surface Technology and Tribology, Faculty of Engineering Technology, University of Twente, P.O. Box 217, 7500 \\ AE Enschede, The Netherlands; m.b.derooij@utwente.nl (M.B.d.R.); d.j.schipper@utwente.nl (D.J.S.) \\ * Correspondence: d.bijani@utwente.nl; Tel.: +31-(0)68-113-3568
}

Received: 29 August 2018; Accepted: 9 October 2018; Published: date

\begin{abstract}
In many industrial applications, a modification of the surface geometry can enhance the tribological behaviour of lubricated sliding contacts. In this paper, the effect of surface texturing on the coefficient of friction in parallel sliding lubricated surfaces is investigated. It is shown that surface texturing can improve film formation and, as a result, the load-carrying capacity as well as a reduction in the coefficient of friction. With the numerical model developed, and by considering cavitation, the effects of shape, depth, size, and the textured area fraction on the frictional behaviour of parallel sliding lubricated contacts under conditions of mixed lubrication is studied. In this article it is shown that the surface texturing can have a beneficial effect, in order to decrease friction.
\end{abstract}

Keywords: mixed lubrication; deterministic asperity model; surface texturing; film thickness; texturing patterns; numerical modelling

\section{Introduction}

There is a growing demand for improving the tribological performance (frictional behaviour) of sliding lubricated contacts in many applications. The first method of reducing friction is to make the surfaces smoother; however, producing a super-smooth surface can be expensive, and it may cause failure [1]. In the case of lubricated contacts, a geometrical modification on the micro-scale of the surfaces can be a potential solution for this problem.

Surface texturing has been widely applied as a well-known method of reducing friction and wear between sliding surfaces separated by a thin lubricant film. Nowadays, due to improvement of texturing techniques it is possible to employ different geometrical patterns of texturing. These surface modification techniques include machining, photoetching, etching techniques, ion beam texturing, and laser texturing [2]. Laser texturing prevails over the other methods as it is efficient, convenient, and controllable for many materials [3]. However, the process parameters should be determined carefully to avoid a bulge at the rims of the textures $[4,5]$.

In recent decades, much effort has been put into understanding the effect of surface texturing in order to improve the tribological performance of lubricated contacts. The cavities can result in an improvement in tribological performance, due to local convergent and divergent gaps, which can generate pressure, even in lubricated parallel sliding flat-on-flat contacts. In the cavities, cavitation may occur. Therefore, three main effects are expected to occur to enhance the tribological performance of lubricated sliding textured surfaces. The first effect is the improvement in hydrodynamic pressure in the contacts, due to the converging wedges constituted by the cavities or pockets. The second is the containment of wear debris inside the cavities, which can be of benefit by removing the wear particles between the two surfaces, to avoid abrasive wear, for instance. The third effect is more sensible in high plastic deformation conditions: the presence of pockets of lubricants due to texturing could have a beneficial influence on the tribological 
behaviour of the contact [6]. A lot of studies relating to surface texturing with respect to friction have focused on the experimental aspect, and theoretical research has recently received more attention [7-10]. However, it is necessary to identify the effect of each of the parameters that define the texturing pattern. Therefore, the most important parameters influencing the frictional behaviour of lubricated contacts need to be identified. By presenting the results as a Stribeck curve, the lubricated contact performance as a function of the operating conditions such as velocity, and texturing parameters such as texture depth, size, and density, can be analysed.

Asperities collide when two solid surfaces of contacts are very close together (see Figure 1). Under these conditions, the friction is determined predominantly by the interaction between the solids at the asperity level where the boundary layers are being sheared. In addition, the lubricant properties have an insignificant or even no influence on friction force. In lubricated contacts, the boundary lubrication mostly occurs when the contact is under high-load and lowvelocity conditions.

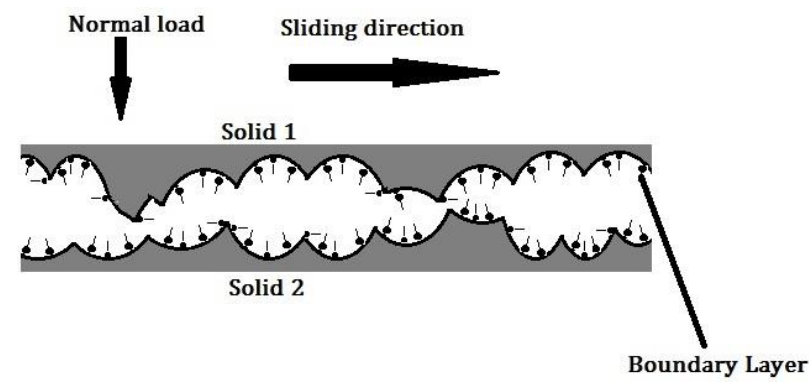

Figure 1. Schematic representation of two surfaces in contact.

Most machine components, such as gears and bearings, are likely to operate in the mixed lubrication regime where asperity contacts exist, and the fluid contributes significantly to carrying the load. Modelling friction under mixed lubrication conditions is a challenging task; much effort has been devoted to this in the past few decades [8,11-13]. There are two types of mixed lubrication models, based on statistical and deterministic contact models. The statistical model uses selected statistical parameters to represent random characteristics of surface roughness. A major shortcoming of these models is their inability to provide detailed information about local affairs, which have an influence on the mechanisms of lubrication and friction. The deterministic model, which uses deterministic information of surface roughness, is another common approach to simulating the behaviour of contacting asperities under mixed lubrication.
This study investigates numerically the effect of surface texturing on the Stribeck curve, i.e., friction in lubricated contacts; in particular, the lubrication regimes of hydrodynamic lubrication and mixed lubrication.

\section{Materials and Methods}

In their study of face seals, Hamilton et al. [14] pointed out that the pressure increases in the converging film regions, whilst facing a reduction in pressure in the diverging film regions likewise in a cavity generated by, for instance, laser surface texturing. When the lubricant enters the cavity zone, by approaching into the end of the divergence zone, the pressure will drop, but by considering the effect of cavitation and the fact that the lubricant pressure cannot reach values lower than the cavitation pressure, the lubricant pressure will reach the lowest value (cavitation pressure). After passing the divergence zone and on reaching the convergence zone, the pressure in the lubricant will start to increase. The pressure generation in the convergence zone can increase to high values, leading to an increase in the load-carrying capacity. Various studies on the tribological behaviour of textured surfaces include numerical $[8,15]$ and experimental investigations on textured surfaces $[16,17]$. Several studies have shown that when using a textured surface, the transition between boundary and hydrodynamic lubrication occurs at a lower velocity than when using a smooth surface $[16,18,19]$.

Etsion and Burstein [7], in their work on the surface texturing of mechanical seals, developed model showing an improvement in seal performance when surface texturing is employed. In their work, one of the seal surfaces was textured with evenly distributed hemispherical micro-dimples.

Wang et al. [20,21] and Kovalchenko et al. [18] have shown the effect of dimples on the friction coefficient and load-carrying capacity. In their studies, several texture patterns were considered, such as spiral grooves, micro-dimple, and square dents. It was reported that the shape, size, texture density, and orientation of the texture patterns played an important role in the film thickness and frictional behaviour of the lubricated contacts. Costa et al. [22] performed an experimental investigation to find the role of surface texturing in generating hydrodynamic pressure in lubricated sliding contacts. They showed that the texturing geometrical parameters such as pattern, shape, density, and the size of the pockets have an influence on the tribological behaviour of contacts, and this can reduce the friction. 
In work by Wang et al. [23], and in Hu and Zhu's mixed lubrication friction model $[11,12]$, the effect of texture distribution patterns on the lubrication performance was investigated in relation to texture direction, the orientation angle of the features, the feature continuity, and the aspect ratio. They showed that narrow short grooves perpendicular to the motion direction, a small orientation angle, and a small ratio of width/length were effective in producing a thick oil film [24]. The optimum value for the different texture geometrical parameters has, in several studies, been investigated analytically $[25,26]$, experimentally $[8,16,17,20,27-29]$, and numerically $[8,15,30,31]$. These studies showed that, for any particular dimple shape, the texture density plays an important role in determining the effectiveness of the texture to increase the load-carrying capacity, and to reduce friction, since the optimum for different shapes might not be at a constant dimple depth. In addition, many researchers investigated the performances of herringbone-grooved journal bearings.

The mixed lubrication $(\mathrm{ML})$ regime is the transition regime between the hydrodynamic lubrication $(\mathrm{HL})$ and the boundary lubrication (BL), and can therefore be seen as a combination of the two, having the properties of both regimes, and the value of the coefficient of friction in the $\mathrm{ML}$ regime varies between the values of the coefficient of friction of the $\mathrm{BL}$ and $\mathrm{HL}$ regimes. Most of the researchers who studied the mixed lubrication regime, employed experimental methods in their investigations (Stribeck [32], Hersey [33], Lenning [34], and Schipper [35]) as opposed to theoretical methods (Patir and Cheng [36,37], Johnson, Greenwood and Poon [38], and Gelinck and Schipper [39] etc.). Patir and Cheng investigated the effect of roughness on the hydrodynamic load by introducing the average Reynolds equation with pressure and shear flow factors; however, their analysis is actually valid for separations that are larger than three times the combined root mean square surface roughness (Rq). In 1972, Johnson, Greenwood, and Poon combined the Greenwood and Williamson [40] theory with the elastohydrodynamic lubrication theory in order to develop a mixed lubrication model in which the load over the lubricated contact is shared between the lubricant film and the asperity contact. Gelinck and Schipper [39] extended this model for line contacts in order to determine the Stribeck curve. Shi and Salant [41] introduced a mixed lubrication model, which considers inter-asperity cavitation and surface shear deformation for soft materials, and showed the occurrence of local cavitation. For such moderately loaded lubricated systems, the Jakobsson-Floberg-Olsson [42,43] cavitation theory is typically used. It is worth mentioning that when the surface tension effect is significant, the Jakobsson-Floberg-Olsson theory is less reliable [44]. A numerical model is developed in the work of Faraon et al. [45]. The model developed here is based on the deterministic mixed lubrication model, in which the local cavitation in the contact zone is considered. This is realised by combining the film thickness calculation results from a previous study [46] with a deterministic asperity contact model to calculate the friction. This model will provide a better understanding with respect to the influence of surface texture features on friction in the mixed lubrication regime.

\section{Mathematical Solution and Modelling}

\section{Deterministic Asperity Model}

In their contact model [40] Greenwood and Williamson, assumed that the asperities are spheres with equal radius, and they employed a Gaussian distribution of the summit heights. However, in reality, the asperity radius is not constant for all asperities in contact. Also, most of the surfaces have a height distribution, which is not Gaussian. Although this model is quite reliable when the aforementioned conditions are applied, the assumption of a Gaussian distribution of equal summits is a drawback in this model. Here, in order to avoid Greenwood and Williamson's assumptions, a deterministic contact model was developed based on measured roughness data (see Figure 2).

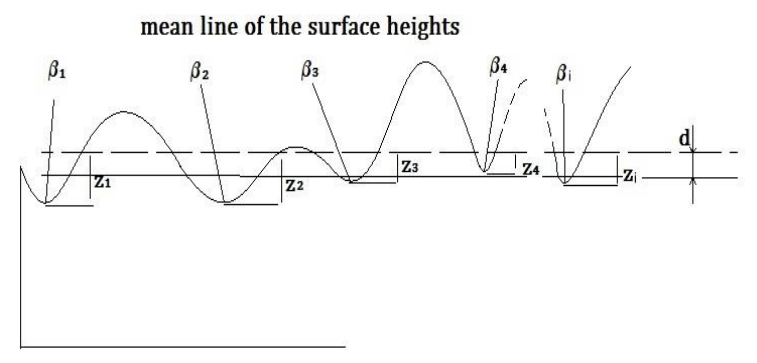

Figure 2. The contact between a rough surface and a flat surface.

Figure 2, shows a schematic illustration of the contact between a rough surface and a flat surface. By adding together the local contributions, it is possible to calculate deterministically the quantities of real contact area, the number of asperities, and the total force carried by the contact, when the separation between the two surfaces $(d)$, is known. In this contact model, it is assumed that the asperities are deforming independently of each other, and that they have 
elliptical shapes. Furthermore, the radii of these ellipsoids are not similar for all asperities (the asperity radii are measured in sliding and in perpendicular direction $\beta_{x i}, \beta_{y i}$ ).

$$
w_{i}=z_{i}-d
$$

In this equation, $z_{i}$ is the individual summit height and $d$ is the separation parameters.

By determining the value of each deformed asperity $\left(w_{i}\right)$ and the total real contact area, and by adding the individual components of each asperity, it is possible to calculate the normal load over the asperities $\left(F_{\mathrm{c}}\right)$. According to Johnson et al. [38], the total normal load on the contact in the $\mathrm{ML}$ regime is carried by the $\mathrm{BL}\left(F_{\mathrm{C}}\right)$ and the $\mathrm{HL}\left(F_{H}\right)$ force component, Therefore:

$$
F_{T}=F_{C}+F_{H}
$$

Based on Equation (2), coefficients $\gamma_{1}$ and $\gamma_{2}$ are introduced [38]:

$$
\gamma_{1}=\frac{F_{T}}{F_{H}}, \gamma_{2}=\frac{F_{T}}{F_{C}}
$$

The two coefficients, $\gamma_{1}$ and $\gamma_{2}$, refer to the $\mathrm{HL}$ component and the $\mathrm{BL}$ component, respectively. The two coefficients $\left(\gamma_{1}\right.$ and $\left.\gamma_{2}\right)$ are mutually dependent through the equation:

$$
1=\frac{1}{\gamma_{1}}+\frac{1}{\gamma_{2}}
$$

In the deterministic asperity contact model, the entire Stribeck curve can be calculated by using the two coefficients $\gamma_{1}$ and $\gamma_{2}$ for the contact between a rigid flat surface against a rough one and the HL theory.

In mixed lubricated contacts, in order to measure the frictional behaviour and to calculate the coefficient of friction of contact, three parameters must be calculated: the load carried by the asperities, the load carried by the fluid, and the lubricant film thickness. Three equations are used to calculate the values of the three aforementioned parameters.

I. The first equation is Equation (2) $\left(F_{T}=F_{C}+F_{H}\right)$.

II. The Reynolds equation.

The Reynolds equation is deduced from the Navier-Stokes equations under the assumption that the gap between the surfaces and the Reynolds number is small. The general Reynolds equation in Cartesian coordinates can be written as Equation (5):

$$
\begin{aligned}
\frac{\partial}{\partial x}\left(\frac{\rho h^{3}}{\eta}\right. & \left.\frac{\partial p}{\partial x}\right)+\frac{\partial}{\partial y}\left(\frac{\rho h^{3}}{\eta} \frac{\partial p}{\partial y}\right) \\
& =6\left(\mathrm{u}_{0}\right) \frac{\partial(\rho h)}{\partial x} \\
& +6 \rho h \frac{\partial\left(\mathrm{u}_{0}\right)}{\partial x}+12 \frac{\partial(\rho h)}{\partial t}
\end{aligned}
$$

By approaching the outlet wall of the cavity, the lubricant enters into a converging zone, which results in an increase in the lubricant pressure. At the entry of the cavity, the lubricant flow diverges, which results, according to the Reynolds Equation (5), in a negative pressure. The negative pressure is suppressed by cavitation; i.e. vapour bubbles are formed in the lubricant. Based on Jakobsson-Floberg-Olsson, the lubrication film is divided into two zones. The first part is with a complete lubricant film; in this region, the Reynolds equation applies. In the second part, cavitation takes place, and a fraction of the lubricant film gap is occupied. Because the vapour bubbles are formed within the void fraction, the pressure throughout the cavitation area is taken as being constant [44].

The Reynolds equation, in a Cartesian coordinate system, can be written as Equation (6) [47]. In this steady-state equation, the mass-conservation and cavitation conditions are also considered.

$$
\begin{aligned}
& \frac{\partial}{\partial x}\left(\frac{h^{3}}{\eta} \frac{\partial(F \varphi)}{\partial x}\right)+\frac{\partial}{\partial y}\left(\frac{h^{3}}{\eta} \frac{\partial(F \varphi)}{\partial y}\right) \\
= & \frac{6 u_{0}}{p_{a}-p_{c}} \frac{\partial((1+(1-F) \varphi) h)}{\partial x}
\end{aligned}
$$

In this equation, $h$ is the film thickness, $\eta$ is the viscosity, $u_{0}$ is the sum velocity, $p_{a}$ is the ambient pressure, $p_{c}$ is the cavitation pressure, $F$ is a dimensionless dependent variable, and $\varphi$ is the cavitation index; for more information, the reader is referred to Xiong and Wang [47].

Three different texture patterns have been investigated in this study: the chevron, the groove, and the triangular pocket. Figure 3 shows the different cavity shapes and the parameters characterizing their geometry. The chevron pocket geometry can be characterised by using two equilateral triangles of different sizes. The midpoint of the altitude line of the triangle in a chevron or triangle pattern is the centre of the unit cell; see also [48]. All patterns have a rectangular cross-sectional profile. The general film thickness formula can be written as Equation (7):

$$
h=h_{0}+h_{\text {macro }}+h_{\text {texture }}
$$

In the case of a flat-flat contact, the macro geometry is omitted and the film thickness Equation (7) reduces to Equation (8) $[48,49]$ :

$$
\frac{h(x, y)}{h_{0}(x, y)}=1+H(x, y)
$$



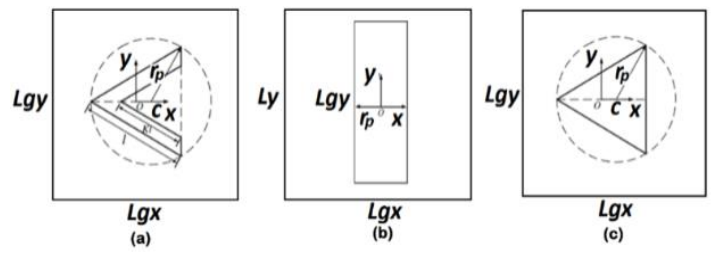

Figure 3. Geometrical scheme of patterns, (a) chevron, (b) groove, (c) triangular pocket (reproduced with the permission of Mingfeng Qiu, Bret R. Minson, Bart Raeymaekers, Tribology International, published by Elsevier, 2013) [48].

In this simulation, $r_{p}$ is the characteristic radius for the triangular and chevron patterns, and the half-width of the grooves.

In order to solve Equation (6) iteratively, the TriDiagonal Matrix Algorithm (TDMA) is used; to reduce the storage needed for calculation, the line-by-line TDMA solver (Patankar [50]) is applied. For a twodimensional problem, the TDMA solution column-bycolumn or row-by-row becomes iterative, and sweeping is done line-by-line, and column-by-column or row-by-row. For three-dimensional problems, the TDMA is applied line-by-line on a selected plane, and then the calculation is moved to the next plane, scanning the domain plane-by-plane [51].

III. The third equation is the force carried by asperities:

$$
F_{\mathrm{c}}=\sum_{i=1}^{N} \frac{2}{3} E^{\prime} R_{i}^{\frac{1}{2}}\left(z_{i}-d\right)^{\frac{3}{2}}
$$

in which $E^{\prime}$ is the combined elasticity modulus, and $R_{i}$ is the reduced radius of the cylinder. The reduced elastic modulus is given by:

$$
\frac{2}{E^{\prime}}=\frac{1-v_{1}^{2}}{E_{1}}+\frac{1-v_{2}^{2}}{E_{2}}
$$

where $E_{1}=E_{2}=E$ and $v_{1}=v_{2}=v$.

Finally, the total friction force $\left(F_{f}\right)$ in the $\mathrm{ML}$ regime is the sum of the friction force of the contacting asperities and the shear force of the lubricant $\left(F_{f h}\right)$ :

$$
F_{f}=\sum_{i=1}^{N} \iint_{A_{c i}} \tau_{c i} d A_{c i}+F_{f H}
$$

where the first term is the friction force due to contacting asperity; $N$ is the number of contacting asperities; $A_{c i}$ is the area of contact of a single asperity; $\tau_{c i}$ is the shear stress at the asperity contact. For the HL component, the friction force can be calculated as:

$$
F_{f H}=\tau_{H} A_{H}
$$

where $A_{H}$ is the contact area of the hydrodynamic component and $\tau_{H}$ is the shear stress of the lubricant. For the contacting asperities, the friction is assumed to be of the Coulomb type, i.e.:

$$
f_{C i}=\frac{\tau_{C i}}{p_{C i}}
$$

with $p_{c i}$ being the average contact pressure on the current asperity. The coefficient of friction $f_{C i}$ is assumed constant for all asperities; therefore, the first term of Equation (13), can be written as:

$$
\sum_{i=1}^{N} \iint_{A_{c i}} f_{C} p_{c i} d A_{c i}=f_{C} F_{C}
$$

where the value of $f_{C}$ is experimentally determined and set in this work at 0.1. Now, the coefficient of friction can be written as:

$$
f=\frac{F_{f}}{F_{T}}=\frac{f_{C} F_{C}+F_{f H}}{F_{T}}
$$

By solving these equations, the coefficient of friction in $M L$ regime can be calculated as explained in Appendix A, (see Figure A1).

\section{Problem Definition and its Solution}

In these calculations, the number of pockets and the distance between the pockets are combined by introducing a new parameter, pitch. The pitch $\left(P_{i}\right)$ for the chevron and groove pockets is calculated using the cavity size:

$$
S=2 \times r_{p}
$$

The pitch in $x$ direction, $P_{i}=\frac{S}{L_{g x}}$

In this study, the influence of different texturing parameters on the frictional behaviour of contacts is investigated. From the previous study on the film thickness [46], it is possible to limit the investigation on texturing parameters and patterns. The effect of the cavity cross-sectional shape is studied [46], and the rectangular cross-section has been found as the more efficient pattern; therefore, in the present study, the calculations will be limited to cavities with a rectangular cross-section (see Figure 4d). In order to find the most efficient pattern, the coefficient of friction calculation will be based on linear groove, chevron, and triangular pockets. The effect of different texturing parameters, such as cavity depth and cavity size as well as pitch, will be investigated. The texturing properties that are applied in this calculation, are presented in (Table 1). In Table 1, as mentioned before, $r_{p}$ is characteristic radius of the pocket in the case of chevrons and triangular pockets and the half-width of the groove, in case of rectangular grooves. More information about the roughness and boundary lubrication parameters are presented in Appendix B. 


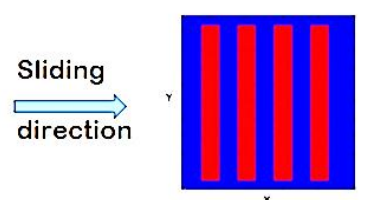

(a)

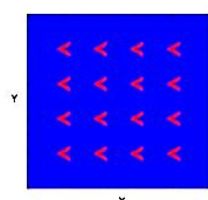

(b)

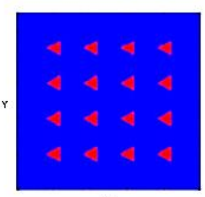

(c)

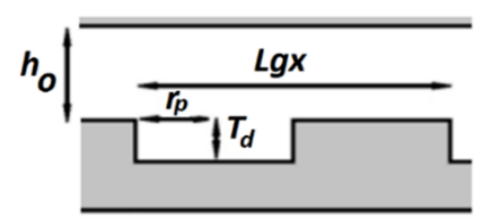

(d)

Figure 4. Schematic illustration of different patterns: (a) Grooves, (b) chevrons, (c) triangular pockets and (d) schematic illustration of cavity profile.

Table 1. Operating conditions.

\begin{tabular}{lc}
\hline \multicolumn{1}{c}{ Parameter } & Value \\
\hline Texture depth, $T_{d}$ & $10 \mu \mathrm{m}$ \\
Texture pitch, $P_{i}$ & 0.4 \\
Cavity size, $\mathrm{S}=2 r_{p}$ & $50 \mu \mathrm{m}$ \\
Normal load & $5 \mathrm{~N}$ \\
Average contact & $0.05 \mathrm{MPa}$ \\
pressure & \\
Dynamic viscosity & $8 \mathrm{mPa} \cdot \mathrm{s}$ \\
Elasticity modulus, $E$ & $210 \mathrm{GPa}$ \\
Poisson's ratio, $v$ & 0.3 \\
\hline
\end{tabular}

\subsection{Comparison of Patterns}

In order to determine the effect of texturing on lubricated contacts with respect to friction, several simulations were carried out using different texturing parameters. The coefficient of friction for grooves was calculated as well as for chevron and triangular pockets parallel to the moving direction. From a previous study [46], simulations indicated that a higher lubricant pressure generation is achievable by closed grooves, because the side leakage for open grooves is greater than for closed grooves; see Figure 4a. Therefore, in this study, closed grooves were chosen to determine their effect on the coefficient of friction.

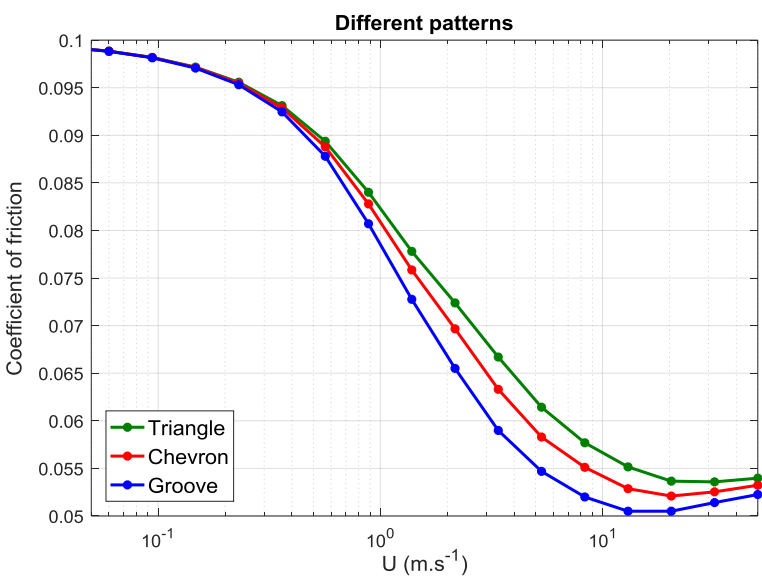

Figure 5. Comparison between different patterns, texturing properties are given in Table

1.

In Figure 5, in the boundary lubrication region when the surfaces start to slide against each other, $\left(U<0.1 \mathrm{~m} \cdot \mathrm{s}^{-1}\right)$, due to the lack of lubricant film in contact, the normal load carries by solid asperities. In this region the coefficient of friction is high and under the influence of the roughness of surfaces, and different texturing patterns have almost the same value.

In parallel sliding contacts, when the velocity is increased the wedge effect caused by the existence of textures over the surface leads to an increase of the lubricant pressure. Moreover, the pressure generation in fluid film results in fewer asperities in contact, and a drop in the coefficient of friction. In this region, the load over the contact carries by lubricant as well as by solid asperities; therefore, the performance of the lubricant film in contact has an influential effect on the coefficient of friction. In a mixed lubrication regime, patterns that are more successful in film formation can cause a greater reduction of friction. The biggest drop in the coefficient of friction in this region belongs to the surfaces with a groove pattern; after that, the chevrons are successful in the case of friction reduction.

In higher velocity range $\left(U>10 \mathrm{~m} \cdot \mathrm{s}^{-1}\right)$, the lubrication regime changes from a mixed to a hydrodynamic lubrication regime. In the hydrodynamic lubrication regime, by increasing the sliding velocity, the shear stress also increases, and a higher shear stress results in a higher coefficient of friction. For those patterns that are more efficient in film formation, the transition point between the mixed and hydrodynamic lubrication regime occurs at a lower velocity; therefore, the friction begins to increase in the lower velocities also. Figure 5 , shows that in a hydrodynamic lubrication regime $\left(U>10 \mathrm{~m} \cdot \mathrm{s}^{-1}\right)$ the coefficient of friction increases more dramatically in the 
case of a groove pattern, and the least sensitive pattern to the velocity increment is a triangular pattern.

When the distances between the texture cells are the same (the pitch is constant) the groove pattern is more effective in generating a local load-carrying capacity because of the higher textured area fraction per unit area. Chevrons are more beneficial than the triangular pockets because of the existence of a longer outlet wall in the cavity zone (Figure 5).

\subsection{Property Effects}

In this section, an optimization of the geometrical parameters is performed. The effect of texture pitch, cavity size, and texture depth on the coefficient of friction for cavities with a rectangular profile (Figure 4) is studied in this optimization. Grooves can be characterised by three geometry parameters, and in the case of chevrons, a fourth parameter is needed to define this pattern; see Figure 6.

Cavity size: $S=2 r_{p}$

Texture depth: $T_{d}$

Texture pitch: $P_{i}=\frac{\mathrm{S}}{L_{g x}}$

Cavity width ratio: $K$

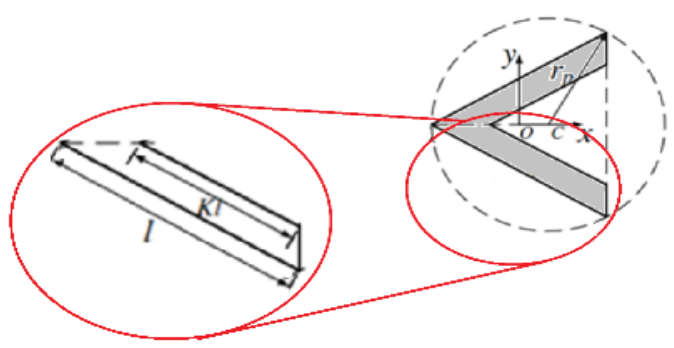

Figure 6. Definition of cavity width ratio; $K$ is the ratio of the inner wall length over the outer wall length.

In the calculations, $K$ is kept constant and is equal to 0.5 .

\subsubsection{The effect of texture depth $\left(T_{d}\right)$}

In Figure 7, simulation results are shown for the coefficient of friction as a function of the texture depth for the groove and chevron pattern. When the depth is tending to zero, there is no film formation because of the absence of the wedge effect in the flat-flat contact and the lack of texturing.

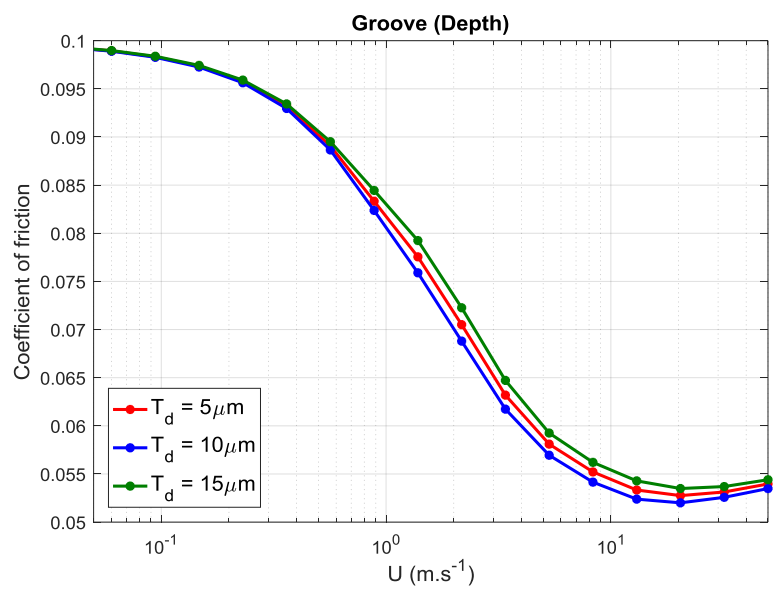

(a)

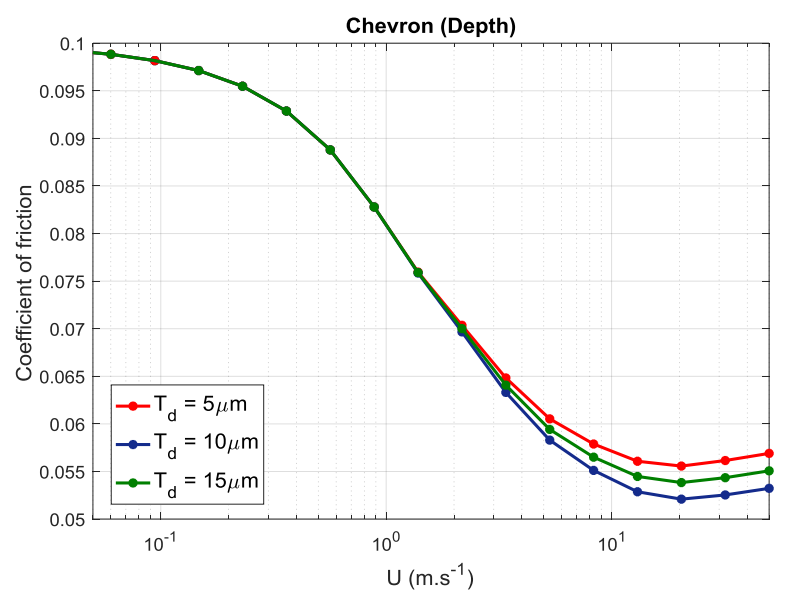

(b)

Figure 7. Coefficient of friction as a function of speed for patterns with different texture depth for: (a) grooves, (b) chevrons.

For both chevron and groove patterns, the fluctuation of texture depth affects the hydrodynamic lubrication component of mixed lubrication. A texture depth that is close to $10 \mu \mathrm{m}$ leads to a reduction in the coefficient of friction due to a film thickness increase. After the texture depth passes the optimum value for the texture depth, i.e. $10 \mu \mathrm{m}$, deeper cavities result in an increase in the coefficient of friction. This phenomenon is caused by the reduction in lubricant film thickness [46]. From Figure 7, it is concluded that when the textured contact is operating near the transition between the $\mathrm{ML}$ and $\mathrm{HL}$ regimes, the texture depth has an influence on the frictional behaviour.

\subsubsection{The Effect of Cavity Size (S)}

The coefficient of friction in lubricated contacts in the $M L$ and $H L$ regimes are influenced by several parameters, as well as by lubricant viscosity and contact pressure. When these parameters have a fixed value it is possible, by changing the geometrical parameters, to 
detect the influence of the size of the surface texturing on the frictional behaviour of the contact. In Figure 8, the effect of the cavity size $(S)$ of the chevron and groove patterns on the coefficient of friction is shown. As found for the texturing depth, an optimum value for the cavity size $(S)$ exists. For sizes tending to zero or which are very large there is no pressure generation, due to the absence of the wedge effect.

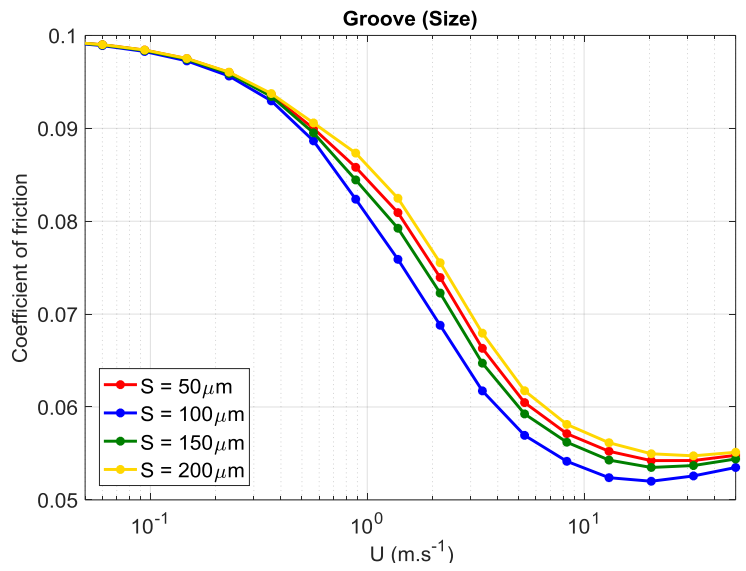

(a)

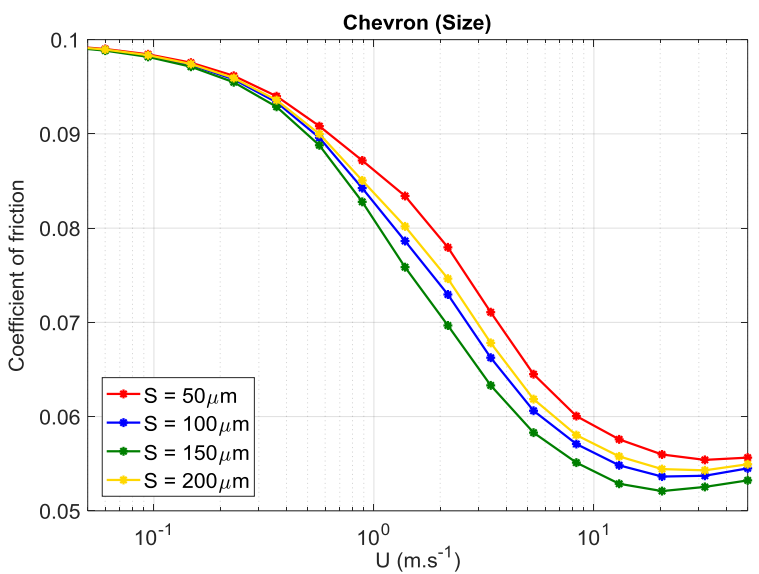

(b)

Figure 8. Coefficient of friction as a function of speed for patterns with different cavity sizes $(S)$ for: (a) grooves, (b) chevrons.

Changing the cavity size will affect the hydrodynamic lubrication component. In the case of grooves (see Figure 8a), increasing the cavity size to $100 \mu \mathrm{m}$, gives rise to a reduction in the coefficient of friction due to the increase in film thickness. After passing the $S=100 \mu \mathrm{m}$, the larger cavities cause a drop in film thickness, resulting in an increase in the coefficient of friction. For higher velocities, the hydrodynamic lubrication is the dominant lubrication mechanism. The lowest coefficient of friction is achievable when the size is approximately $100 \mu \mathrm{m}$. In the case of chevrons (see Figure 8b), it emerges from the previous study [46], that optimum value for the film thickness is achievable when the size of chevron cavity is around $150 \mu \mathrm{m}$. Therefore, the lowest coefficient of friction can be obtained when cavities with $S=$ $150 \mu \mathrm{m}$ are applied.

\subsubsection{The Effect of Texture Pitch $\left(P_{i}\right)$}

By adjusting the pitch parameter, the quantity of the chevron pockets and grooves over the surface area will undergo a change. When the pitch parameter value is equal to one, as well as when the pitch is zero, there is no textured area. Therefore, there should be a specific value for the pitch parameter for each pattern in which the texturing has a maximum effect on the frictional behaviour. By applying a pattern with a pitch value around that specific value, a reduction in the coefficient of friction will be expected. In Figure 9, the simulation results show the variation of coefficient of friction based on the texture pitch for groove and chevron patterns.

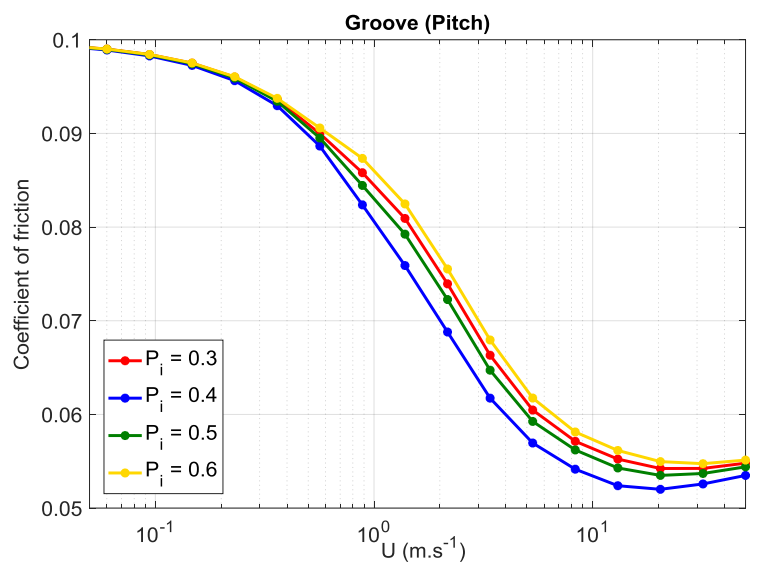

(a)

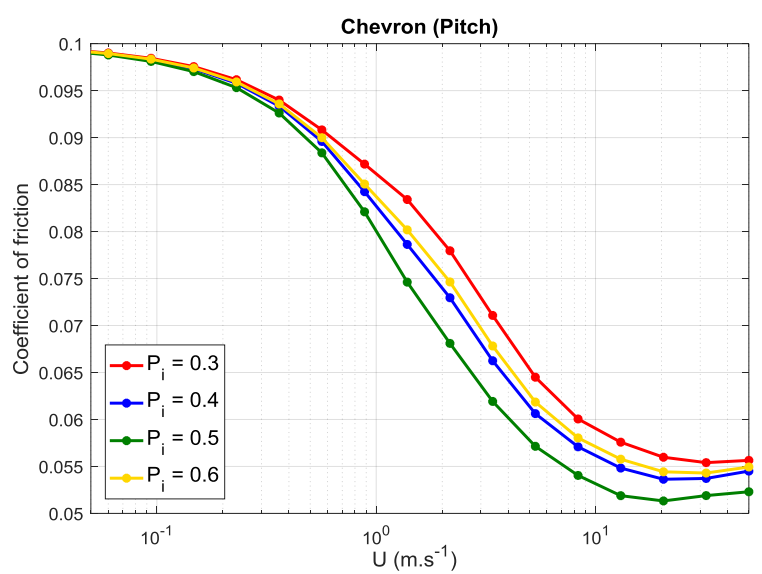

(b)

Figure 9. Coefficient of friction as a function of speed for patterns with different texture pitch for: (a) grooves, (b) chevrons. 
Due to the high texture area coverage when grooves are applied, a low coefficient of friction is achievable with a lower value for the pitch parameter than with chevrons.

In the case of grooves (see Figure 9a), increasing the texture pitch value up to 0.4 leads to a reduction of the coefficient of friction due to the existence of a thicker lubricant film. For pitch values in excess of 0.4 , the coefficient of friction increases due to a decrease in film thickness [46]. These results show that the texture pitch has an influence on the hydrodynamic lubrication component of mixed lubrication. For high velocities, the hydrodynamic lubrication component is the dominant factor in the frictional behaviour, and the lowest coefficient of friction is achievable when the pitch is equal to 0.4 .

In the case of chevrons (see Figure 9b), the increase in texture pitch leads to the reduction in friction. The optimum value is reached for a pitch of 0.5 , due to a thicker film thickness. As a result, the lowest coefficient of friction is obtained when the pitch value is around 0.5 .

\section{Conclusion}

The aim of this research was to study and predict the effect of surface texturing on the coefficient of friction for parallel sliding contacts. In order to obtain a better understanding of the friction phenomenon, a numerical model has been developed. Simulations of textured surfaces with different patterns and different texture properties are performed to determine the frictional behaviours of lubricated parallel sliding contacts. For the mixed lubrication model, the hydrodynamic film thickness is calculated based on a numerical model. The film thickness model is a numerical algorithm based on the Reynolds equation, along with the Elrod cavitation algorithm. The effect of several parameters on the frictional behaviour of lubricated parallel siding contacts, such as pattern type, depth, size and texture pitch, have been studied. The results can be summarised as:

1. Of the patterns studied, the groove pattern shows the lowest coefficient of friction. When the distances between texture cells are the same, i.e. the pitch is constant, the groove pattern is more successful in generating a load-carrying capacity, because of the higher textured area fraction per unit area. Chevrons are more beneficial than triangular pockets.

2. For both chevron and groove patterns, texturing parameters such as depth, pitch, and size values have an influence on the hydrodynamic lubrication component in the mixed lubrication regime.

3. An increase of the texture depth to a specific value of this parameter for different patterns leads to a reduction of coefficients of friction due to an increase in film thickness.

4. In the case of a groove pattern, the optimum cavity size is approximately $100 \mu \mathrm{m}$, and in the case of chevrons, the optimum cavity size is approximately $150 \mu \mathrm{m}$.

5. The optimum depth for different patterns is in the range of $10 \mu \mathrm{m}$.

6. The optimum value for the texture pitch is 0.4 for the groove pattern, and 0.5 for the chevron patterned surfaces.

Author Contributions: Conceptualization, D.J.S., E.L.D. and D.B.; Methodology, D.J.S. and D.B.; Software, D.B. and A.A.; Validation, D.B. and D.J.S.; Formal Analysis, D.B. and D.J.S.; Investigation, D.B. and D.J.S.; WritingOriginal Draft Preparation, D.B.; Writing-Review \& Editing, D.B., E.L.D., M.B.d.R. and D.J.S.; Supervision, D.J. S.

Funding: This research was funded by Materials Innovation Institute (M2i) grant number M21.1.11448. Conflicts of Interest: The authors declare no conflict of interest.

\section{Nomenclature}

$\eta \quad$ Dynamic viscosity

$\mathrm{Pa} \cdot \mathrm{s}$

$\rho \quad$ Density

$\varphi$ Cavitation dimensionless variable

$\gamma_{1}$ Adaption parameter for hydrodynamic component in ML

$\gamma_{2} \quad$ Adaption parameter for asperity contact

$v \quad$ Poisson's ratio

$\tau_{C} \quad$ Shear stress of asperity contact

$A_{C} \quad$ Real area of asperity contact

d Separation

E Elasticity modulus

$E^{\prime} \quad$ Reduced elasticity modulus

$f \quad$ Coefficient of friction

$f_{C} \quad$ Coefficient of friction in $\mathrm{BL}$ regime

$F \quad$ Elrod switch function

$F_{f} \quad$ Friction force

$F_{f H} \quad$ Hydrodynamic friction force

$F_{N}$ Dimensionless total normal load

$F_{T} \quad$ Normal load on the contact
$\mathrm{Pa}$

$\mathrm{m}^{2}$

$\mathrm{kg} \cdot \mathrm{m}^{-3}$

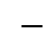

$\mathrm{m}$

$\mathrm{Pa}$

$\mathrm{Pa}$

$-$

$-$

$-$

$\mathrm{N}$

$\mathrm{N}$

$-$

$\mathrm{N}$ 
$F_{C} \quad$ Load carried by the asperities

$F_{H}$ Load carried by the hydrodynamic component

$h \quad$ Film thickness

$h_{0} \quad$ Minimum film thickness

$H$ Dimensionless local depth of textured surface

$L_{g x} \quad$ Texture cell length in the $x$ direction

$L_{x} \quad$ Textured area in the $x$-direction

$L_{y} \quad$ Textured area in the $y$-direction

$P_{i} \quad$ Texture pitch

$p \quad$ Pressure

$P \quad$ Dimensionless pressure

$P_{a} \quad$ Average contact pressure

$p_{a} \quad$ Ambient pressure

$p_{c} \quad$ Cavitation pressure

$p_{T} \quad$ Total pressure

$p_{C} \quad$ Asperity pressure
$\mathrm{N}$

$\mathrm{N}$

$\mathrm{m}$

$\mathrm{m}$

m

$\mathrm{m}$

$\mathrm{m}$

$-$

$\mathrm{Pa}$

$\mathrm{Pa}$

$\mathrm{Pa}$

$\mathrm{Pa}$

$\mathrm{Pa}$

$\mathrm{Pa}$
$p_{H} \quad$ Hydrodynamic pressure $\quad \mathrm{Pa}$

$r_{p} \quad$ Cavity characteristic radius $\mathrm{m}$

$R_{i} \quad$ Reduced radius of cylinder $\quad \mathrm{m}$

$S \quad$ Cavity size $=2 r_{p} \quad \mathrm{~m}$

$T_{d} \quad$ Texture depth m

$U_{0} \quad$ Sum velocity $\mathrm{m} . \mathrm{s}^{-1}$

$X$ Dimensionless Cartesian coordinate

$Y \quad$ Dimensionless Cartesian coordinate

$w_{i} \quad$ compliance of an asperity m

$z_{i} \quad$ Asperity height m

Appendix A. Mixed Lubrication Model Algorithm

As mentioned in Section 3, the coefficient of friction in the $M L$ regime can be calculated as explained in the chart presented below, (see Figure A-1). 


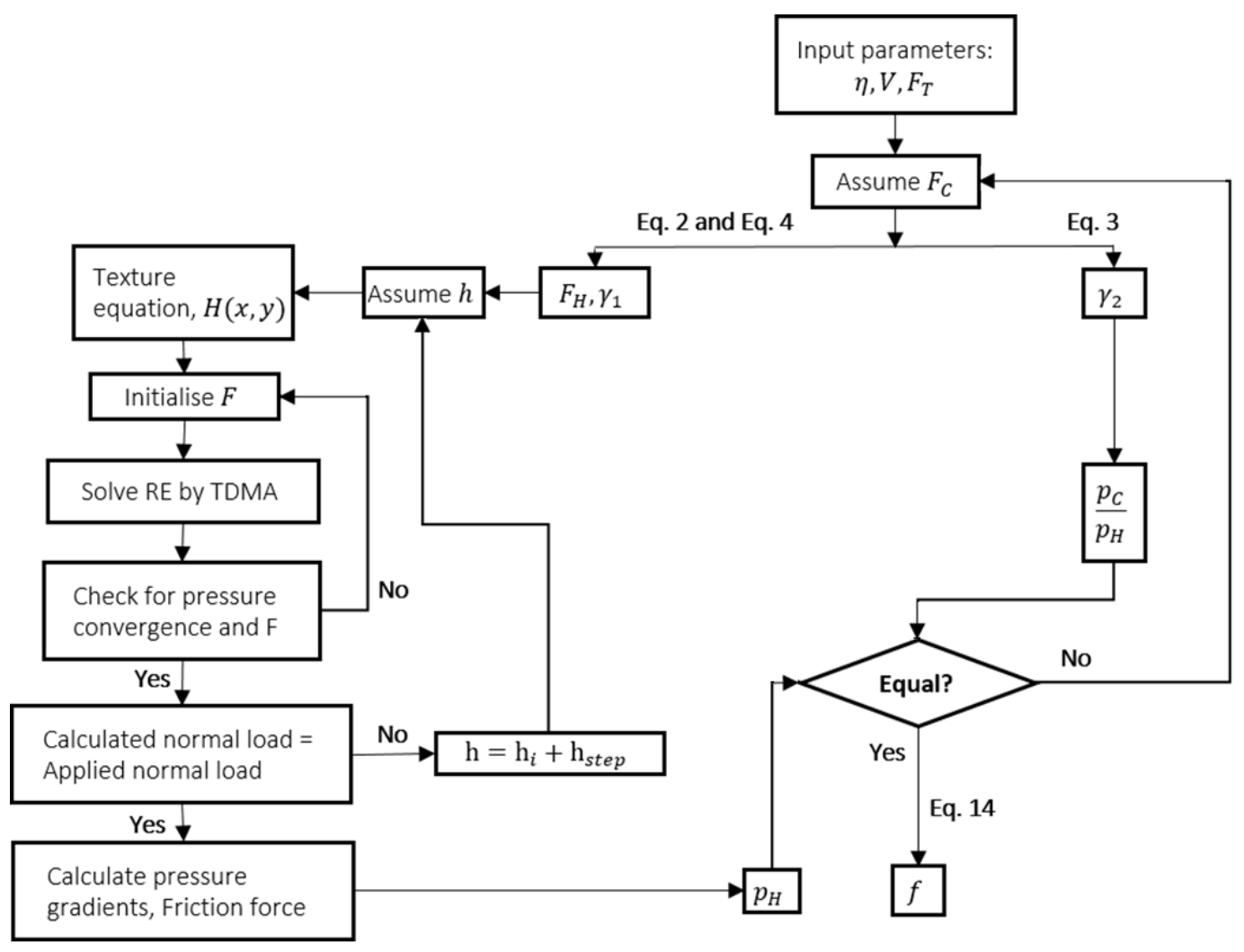

Figure A-1. Mixed lubrication model algorithm.

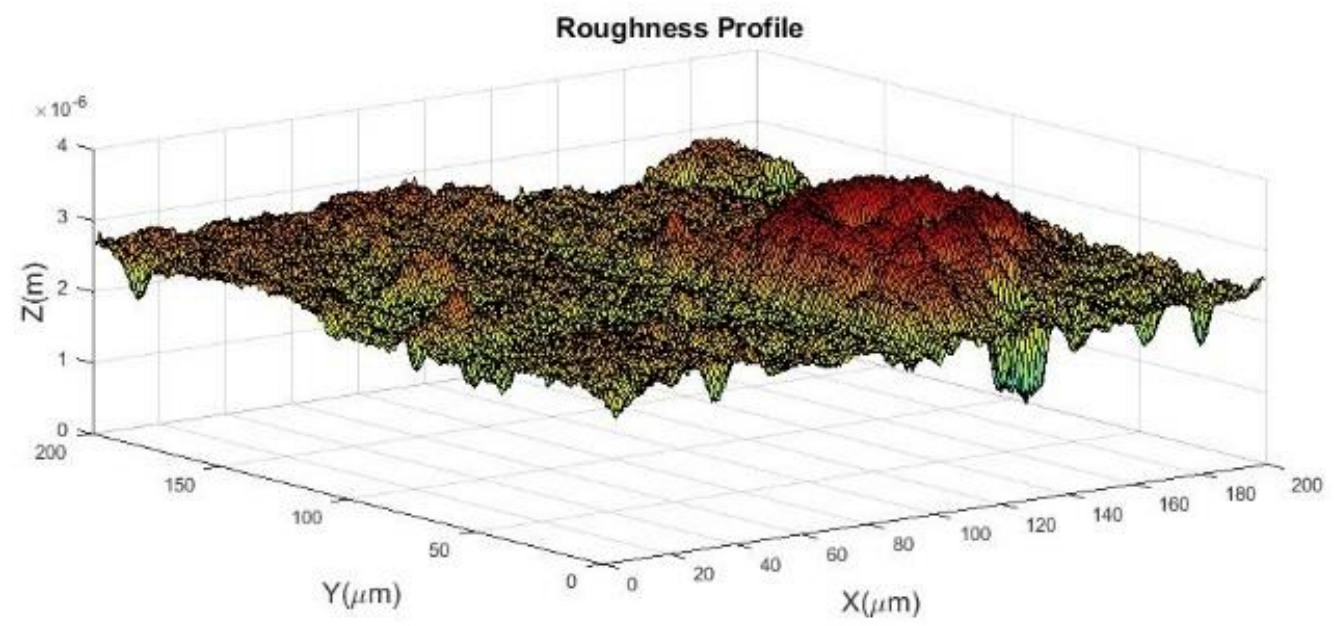

Figure A-3. Roughness measurement surface profile based on the measured roughness by Laser Scanning Microscope measurements, in this case $R_{a}=0.11 \mu \mathrm{m}$. 


\section{Appendix B. Determination of Roughness Parameters}

In this study, calculations were performed using the roughness measured by images extracted from the laser microscope; these images are obtained from roughness height measurements. In this appendix, one of these images is shown as an example and the equations for calculating the roughness parameters are presented as well.

\section{Appendix B.1. Roughness Measurement}

In order to calculate the boundary lubrication component, the roughness measurement is essential. As mentioned in the article, in the case of the deterministic approach, the real measured height of asperities is needed to calculate the separation between the opposing surfaces. In order to achieve the height of the asperities, the surface topography for textured surfaces is measured by using microscopic images. These analyses have been performed using a Keyence Color 3D Scanning Microscope, which uses a violet laser. The result from a roughness measurement in the case of a chevron textured sample is presented in Figure A-2 (standard lens $50 \times$ is employed).

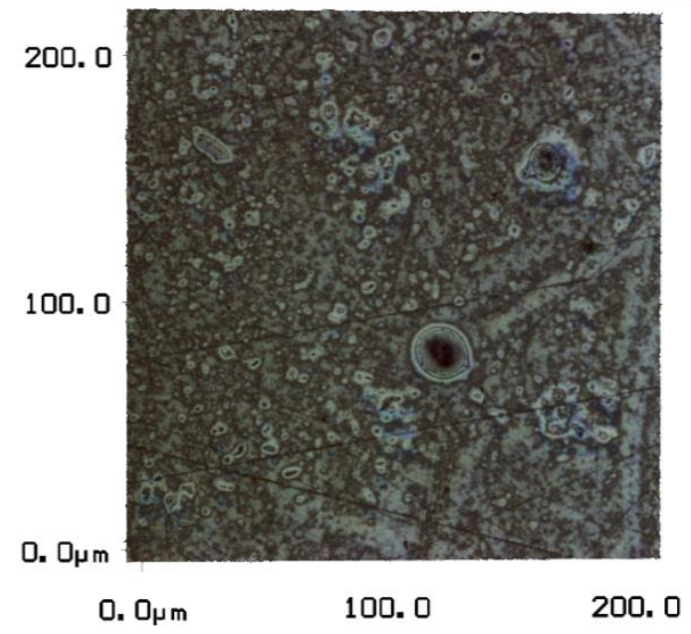

Figure A-2. Laser scanning microscope image of surface.

The 3D illustration is calculated by using the data measured for the surface roughness, (see Figure A-3).

To compare the measured roughness with the Gaussian roughness distribution, the probability density of asperities against the dimensionless asperity height is shown in Figure A-4. In this figure the Gaussian probability density distribution of one surface is shown with the red line and blue bars present the real measured probability density distribution.

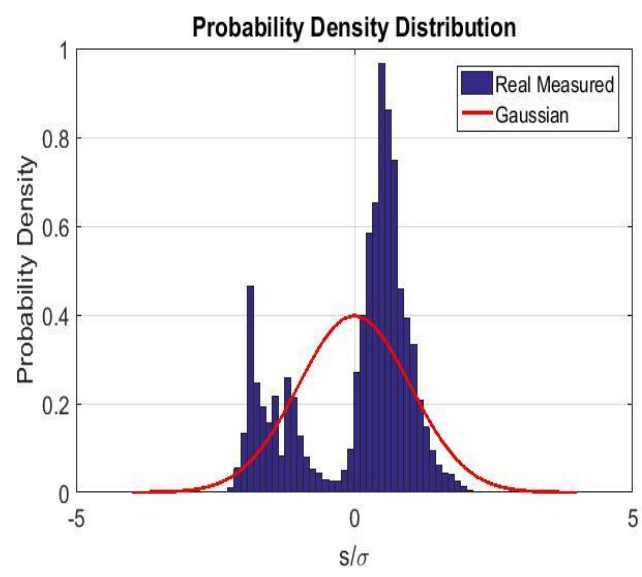

Figure A-4. Distribution of surface heights and the radii of each asperity as a function of the dimensionless asperity height $(s / \sigma)$.

The definition of a summit in this work is that it is higher than its eight neighbour points (see Figure A-5).

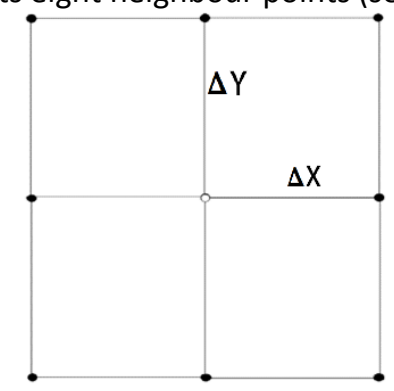

Figure A-5. Definition of a summit.

The radius of an asperity is calculated using the three-point definition:

$$
\begin{aligned}
\beta_{x}^{-1} & =\frac{z_{x-\Delta x, y}-2 z_{x, y}+z_{x+\Delta x, y}}{\Delta x^{2}} \\
\beta_{y}^{-1} & =\frac{z_{x-\Delta x, y}-2 z_{x, y}+z_{x+\Delta x, y}}{\Delta y^{2}}
\end{aligned}
$$

where $\beta_{x}$ and $\beta_{y}$ are the radii in, respectively, the $x$ and the $y$ directions, $z_{x, y}$ is the local surface height at location $(x, y)$ and $\Delta x, \Delta y$ are the step or pixel size. The combined summit radius $\beta_{i}$ of the radii in the two perpendicular directions $\beta_{x}$ and $\beta_{y}$ is obtained by:

$$
\beta_{i}=\sqrt{\beta_{x i} \cdot \beta_{y i}}
$$

The value of $\bar{\beta}$ used in the calculations is the average summit radius:

$$
\bar{\beta}=\frac{1}{n} \sum_{i=1}^{n} \beta_{i}
$$


The calculated average radius of asperity from Figure $A 3$ is equal to $4.8 \times 10^{-8} \mathrm{~m}$.

\section{References}

[1] Hirst, W. Scuffing and its prevention. Chart Mech. Eng. 1974, 21, 88-92.

[2] Wakuda, M.; Yamauchi, Y.; Kanzaki, S.; Yasuda, Y. Effect of surface texturing on friction reduction between ceramic and steel materials under lubricated sliding contact. Wear 2003, 254, 356363.

[3] Wang, W.-Z.; Huang, Z. Shen, D. Kong, L.; Li, S. The effect of triangle-shaped surface textures on the performance of the lubricated point-contacts. J. Tribol. 2013, doi:10.1115/1.4023206.

[4] Baumgart, P.; Krajnovich, D.J.; Nguyen, T.A.; Tam, A.G. A new laser texturing technique for high performance magnetic disk drives. IEEE Trans. Magn. 1995, 31, 2946-2951.

[5] Geiger, M.; Roth, S.; Becker, W. Influence of laserproduced microstructures on the tribological behaviour of ceramics. Surf. Coat. Technol. 1998, 100-101, 17-22.

[6] Steinhoff, K.; Rasp, W.; Pawelski, O. Development of deterministic-stochastic surface structures to improve the tribological conditions of sheet forming processes. J. Mater. Process. Technol. 1996, 60, 355-361.

[7] Etsion, I.; Burstein, L. A Model for Mechanical Seals with Regular Microsurface Structure. Tribol. Trans. 1996, 39, 677-683.

[8] Etsion, I.; Kligerman, Y.; Halperin, G. Analytical and Experimental Investigation of Laser-Textured Mechanical Seal Faces. Tribol. Trans. 1999, 42, 511-516.

[9] Kovalchenko, A.; Ajayi, O.; Erdemir, A.; Fenske, G.; Etsion, I. The effect of laser surface texturing on transitions in lubrication regimes during unidirectional sliding contact. Tribol. Int. 2005, 38, 219-225.

[10] Dobrica, M.B.; Fillon, M.; Pascovici, M.D.; Cicone, T. Optimizing surface texture for hydrodynamic lubricated contacts using a mass-conserving numerical approach. Proc. Inst. Mech. Eng. 2010, 224, 737-750.

[11] Zhu, D.; Hu, Y.-Z. A computer program package for the prediction of EHL and mixed lubrication characteristics, friction, subsurface stresses and flash temperatures based on measured 3-d surface roughness. Tribol. Trans. 2001, 44, 383390.
[12] Hu, Y.Z.; Zhu, D. A full numerical solution to the mixed lubrication in point contacts. J. Tribol. 2000, 122, 1-9.

[13] Zhu, D.; Cheng, H.S.; Hamrock, B.J. Effect of surface roughness on pressure spike and film constriction in elastohydrodynamically lubricated line contacts. Tribol. Trans. 1990, 33, 267-273.

[14] Hamilton, D.B.; Walowit, J.A.; Allen, C.M. A theory of lubrication by microirregularities. J. Basic Eng. 1966, 88, 177-185.

[15] Ronen, A.; Etsion, I.; Kligerman, Y. Frictionreducing surface-texturing in reciprocating automotive components. Tribol. Trans. 2001, 44, 359-366.

[16] Galda, L.; Pawlus, P.; Sep, J. Dimples shape and distribution effect on characteristics of Stribeck curve. Tribol. Int. 2009, 42, 1505-1512.

[17] Qiu, Y.; Khonsari, M.M. Experimental investigation of tribological performance of laser textured stainless steel rings. Tribol. Int. 2011, 44, 635-644.

[18] Kovalchenko, A.; Ajayi, O.; Erdemir, A.; Fenske, G.; Etsion, I. The effect of laser texturing of steel surfaces and speed-load parameters on the transition of lubrication regime from boundary to hydrodynamic. Tribol. Trans. 2004, 47, 299-307.

[19] Raeymaekers, B., Etsion, I.; Talke, F.E. Enhancing tribological performance of the magnetic tape/guide interface by laser surface texturing. Tribol. Lett. 2007, 27, 89-95.

[20] Wang, X.; Kato, K.; Adachi, K.; Aizawa, K. Loads carrying capacity map for the surface texture design of SiC thrust bearing sliding in water. Tribol. Int. 2003, 36, 189-197.

[21] Wang, X.; Kato, K.; Adachi, K.; Aizawa, K. The effect of laser texturing of $\mathrm{SiC}$ surface on the critical load for the transition of water lubrication mode from hydrodynamic to mixed. Tribol. Int. 2001, 34, 703711.

[22] Costa, H.L.; Hutchings, I.M. Hydrodynamic lubrication of textured steel surfaces under reciprocating sliding conditions. Tribol. Int. 2007, 40, 1227-1238.

[23] Wang, Q.J. Zhu, D. Virtual texturing: Modeling the performance of lubricated contacts of engineered surfaces. J. Tribol. 2005, 127, 722-728.

[24] Ren, N.; Nanbu, T.; Yasuda, Y.; Zhu, D.; Wang, Q. Micro textures in concentrated-conformalcontact lubrication: Effect of distribution patterns. Tribol. Lett. 2007, 28, 275-285.

[25] Rahmani, R.; Shirvani, A.; Shirvani, H. Optimization of partially textured parallel thrust bearings with square-shaped micro-dimples. Tribol. Trans. 2007, 50, 401-406. 
[26] Pascovici, M.D.; Cicone, T.; Fillon, M.; Dobrica, M.B. Analytical investigation of a partially textured parallel slider. Proc. Inst. Mech. Eng. 2009, 223, 151-158.

[27] Wang, X.; Adachi, K.; Otsuka, K.; Kato, K. Optimization of the surface texture for silicon carbide sliding in water. Appl. Surf. Sci. 2006, 253, 1282-1286.

[28] Wang, X.; Kato, K. Improving the anti-seizure ability of SiC seal in water with RIE texturing. Tribol. Lett. 2003, 14, 275-280.

[29] Nakano, M.; Korenaga, A.; Korenaga, A.; Miyake, K.; Murakami, T.; Ando, Y.; Usami, H.; Sasaki, S. Applying micro-texture to cast iron surfaces to reduce the friction coefficient under lubricated conditions. Tribol. Lett. 2007, 28, 131-137.

[30] Raeymaekers, B.; Etsion, I. Talke, F.E. A model for magnetic tape/guide friction reduction by laser surface texturing. Tribol. Lett. 2007, 28, 9-17.

[31] Kligerman, Y. and I. Etsion, Analysis of the hydrodynamic effects in a surface textured circumferential gas seal. Tribol. Trans. 2001, 44, 472-478.

[32] Stribeck, R., Die Wesentlichen Eigenschaften der Gleit- Und Rollenlager (The Basic Properties of Sliding and Rolling Bearings). Zeitschrift des Vereins Deutscher Ingenieure, 1902, (36): p. 13411348.

[33] Hersey, M.D., The laws of lubrication of horizontal journal bearings. J. Wash. Acad. Sci., 1914, 4(1), 542-552.

[34] Lenning, R.L., The transition from boundary to mixed friction. J. Amer. Lubr. Eng. 1960, p. 575.

[35] Schipper, D.J. Transitions in the Lubrication of Concentrated Contacts. Ph.D. Theses, University of Twente, Enschede, Sweden, 1988.

[36] Patir, N.; Cheng, H.S. Application of Average Flow Model to Lubrication Between Rough Sliding Surfaces. J. Lubr. Technol. 1978, doi:10.1115/1.3453103.

[37] Patir, N.; Cheng, H.S. An Average Flow Model for Determining Effects of Three-Dimensional Roughness on Partial Hydrodynamic Lubrication. J. Lubr. Technol. 1978, 100, 12-17.

[38] Johnson, K.L.; Greenwood, J.A.; Poon, S.Y. A simple theory of asperity contact in elastohydrodynamic lubrication. Wear 1972, 19, 91-108.

[39] Gelinck, E.R.M.; Schipper, D.J. Calculation of Stribeck curves for line contacts. Tribol. Int. 2000, 33, 175-181.
[40] Greenwood, J.A.; Williamson, J.B.P. Contact of Nominally Flat Surfaces. Proc. R. Soc. Lond. Ser. A Math. Phys. Sci. 1966, 295, 300-319.

[41] Shi, F.; Salant, R.F. A mixed soft elastohydrodynamic lubrication model with interasperity cavitation and surface shear deformation. J. Tribol. 2000, 122, 308-316.

[42] Jakobsson, B.; Floberg, L. The Finite Journal Bearing, Considering Vapourization: (Das Gleitlager von Endlicher Breite mit Verdampfung); Gumpert: Göteborg, Sweden, 1957.

[43] Olsson, K.O. Cavitation in Dynamically Loaded Bearings; Chalmers University of Technology: Göteborg, Sweden, 1965.

[44] Coyne, J.C.; Elrod, J.H.G. Conditions for the rupture of a lubricating film. Part I: Theoretical model. J. Lubr. Technol. 1970, 92, 451-456.

[45] Faraon, I.C. Mixed Lubricated Line Contacts, Ph.D. Thesis, University of Twente, Enschede, The Netherlands, 2005.

[46] Bijani, D.; Deladi, L.E.; Schipper, D.J. The Influence of Surface Texturing on the Film Thickness in Parallel Sliding Surfaces. In Proceedings of the 20th International Colloquium Tribology, Stuttgart/Ostfildern, Germany, 12-14 January 2016.

[47] Xiong, S.; Wang, Q.J. Steady-state hydrodynamic lubrication modeled with the payvar-salant mass conservation model. J. Tribol. 2012, doi:10.1115/1.4006615.

[48] Qiu, M.; Minson, B.R.; Raeymaekers, B. The effect of texture shape on the friction coefficient and stiffness of gas-lubricated parallel slider bearings. Tribol. Int. 2013, 67, 278-288.

[49] Qiu, M.; Delic, A.; Raeymaekers, B. The effect of texture shape on the load-carrying capacity of gaslubricated parallel slider bearings. Tribol. Lett. 2012, 48, 315-327.

[50] Patankar, S.V. Numerical Heat Transfer and Fluid Flow, Series in Computational Methods in Mechanics and Thermal Sciences; Patankar, S.V., Ed.; Hemisphere Pub. Corp.: Washington, DC, USA, 1980.

[51] Versteeg, H.K.; Malalasekera, W. An Introduction to Computational Fluid Dynamics-The Finite Volume Method. Longman Group Ltd.: Harlow, UK, 1995. 


\title{
Paper D
}

\section{The influence of surface texturing on the frictional behaviour in starved lubricated parallel sliding contacts}

\author{
Dariush Bijani ${ }^{1, *}$, Elena L. Deladi ${ }^{2}$, Matthijn B. de Rooij ${ }^{3}$ and Dirk J. Schipper ${ }^{3}$
}

Lubricants 2019

lubricants

MDPI

Article

The Influence of Surface Texturing on the

Frictional Behaviour in Starved Lubricated

Parallel Sliding Contacts

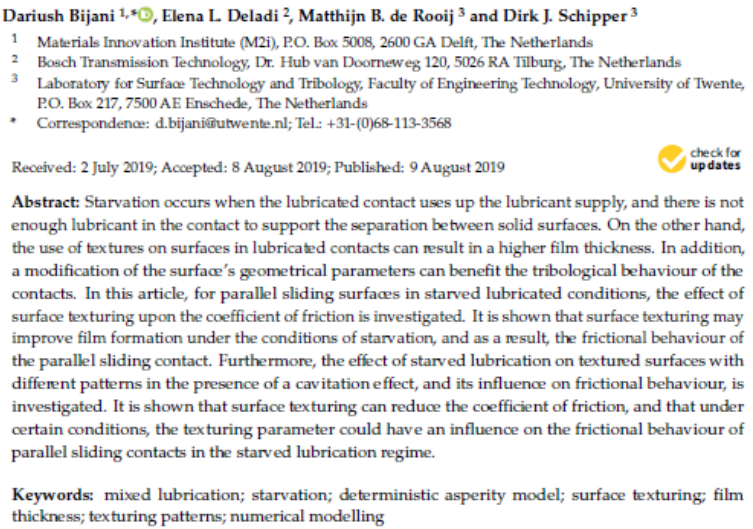

thickness; texturing patterns; numerical modelling

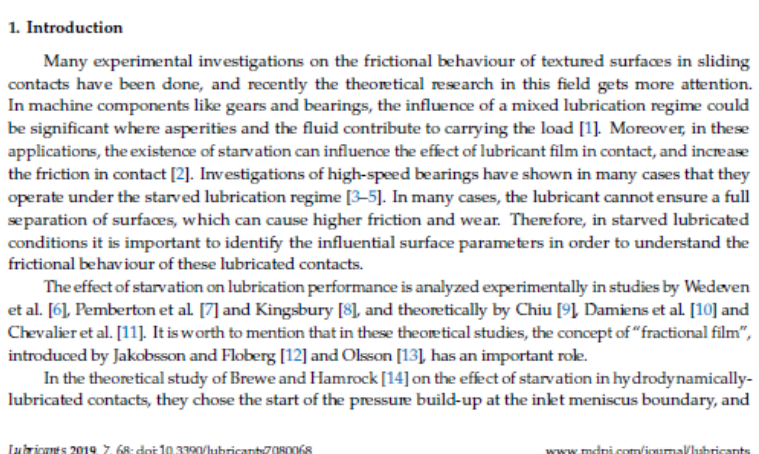





\title{
The Influence of Surface Texturing on the Frictional Behaviour in Starved Lubricated Parallel Sliding Contacts
}

\author{
Dariush Bijani ${ }^{1, *}$, Elena L. Deladi ${ }^{2}$, Matthijn B. de Rooij ${ }^{3}$ and Dirk J. Schipper ${ }^{3}$ \\ 1 Materials Innovation Institute (M2i), P.O. Box 5008, 2600 GA Delft, The Netherlands \\ 2 Bosch Transmission Technology, Dr. Hub van Doorneweg 120, 5026 RA Tilburg, The Netherlands \\ 3 Laboratory for Surface Technology and Tribology, Faculty of Engineering Technology, \\ University of Twente, P.O. Box 217, 7500 AE Enschede, The Netherlands \\ * Correspondence: d.bijani@utwente.nl; Tel.: +31-(0)68-113-3568
}

Received: 2 July 2019; Accepted: 8 August 2019; Published: date

\begin{abstract}
Starvation occurs when the lubricated contact uses up the lubricant supply, and there is not enough lubricant in the contact to support the separation between solid surfaces. On the other hand, the use of textures on surfaces in lubricated contacts can result in a higher film thickness. In addition, a modification of the surface's geometrical parameters can benefit the tribological behaviour of the contacts. In this article, for parallel sliding surfaces in starved lubricated conditions, the effect of surface texturing upon the coefficient of friction is investigated. It is shown that surface texturing may improve film formation under the conditions of starvation, and as a result, the frictional behaviour of the parallel sliding contact. Furthermore, the effect of starved lubrication on textured surfaces with different patterns in the presence of a cavitation effect, and its influence on frictional behaviour, is investigated. It is shown that surface texturing can reduce the coefficient of friction, and that under certain conditions, the texturing parameter could have an influence on the frictional behaviour of parallel sliding contacts in the starved lubrication regime.
\end{abstract}

Keywords: mixed lubrication; starvation; deterministic asperity model; surface texturing; film thickness; texturing patterns; numerical modelling

\section{Introduction}

Many experimental investigations on the frictional behaviour of textured surfaces in sliding contacts have been performed, and recently the theoretical research in this field has been getting more attention. In machine components like gears and bearings, the influence of a mixed lubrication regime could be significant where asperities and the fluid contribute to carrying the load [1]. Moreover, in these applications, the existence of starvation can influence the effect of lubricant film in contact, and increase the friction in contact [2]. Investigations of high-speed bearings have shown in many cases that they operate under the starved lubrication regime [3-5]. In many cases, the lubricant cannot ensure a full separation of surfaces, which can cause higher friction and wear. Therefore, in starved lubricated conditions it is important to identify the influential surface parameters in order to understand the frictional behaviour of these lubricated contacts.
The effect of starvation on lubrication performance is analyzed experimentally in studies by Wedeven et al. [6], Pemberton et al. [7] and Kingsbury [8], and theoretically by Chiu [9], Damiens et al. [10] and Chevalier et al. [11] . It is worth mentioning the concept of "fractional film" introduced by Jakobsson and Floberg [12] and Olsson [13] has an important role in these theoretical studies.

In the theoretical study of the effect of starvation in hydrodynamically-lubricated contacts, Brewe and Hamrock [14] chose the start of the pressure build-up at the inlet meniscus boundary, and by employing a systematic reduction of the fluid inlet level, they observed an increase in the contact pressure for a specified film thickness. By considering a wide range of geometry parameters, they solved the Reynolds equation to simulate the film thickness in the contact area. Moreover, in their study the film thickness formula in the hydrodynamic lubrication regime is modified to incorporate the starvation effect into it. In the work of Boness [15] on the cage and roller slip, it 
was shown experimentally that the oil supply can have a significant effect upon the cage and roller motion, and that limiting the oil supply decreases the amount of slip. Chevalier et al. [16] employed an iso-viscous hydrodynamic model to analyse a non-deformable body; in their study the flow continuity equation is based upon the Elrod $[17,18]$ theory. They conclude that the inlet film shape could affect the film thickness. In Cann and Lubrecht [19], the relationship between the film thickness, velocity, load and viscosity, was the focus of investigation.

Investigations on starved lubrication show that the employment on surface modification methods could be a practical and efficient method for the reduction of friction. One of the first rational methods of decreasing the friction can be by a reduction of roughness, making the surfaces smooth; however, producing an extra smooth surface is expensive [20]. In this case, surface texturing proved to be a reliable method to influence the frictional behaviour in the contacts. A well-designed use of this technique can modify the hydrodynamic component of mixed lubrication, which results in the enhancement of several tribological parameters, such as load-carrying capacity and friction coefficient [21-29]. In general, when the temperature increases, the shear stress of the boundary layer decreases except when the temperature is passing from the melting point of boundary layer. Moreover an increase in temperature of the parts due to interaction between asperities can change the situation from effective lubrication to high wear [30]. In work of Kango et al. [31], based on the Reynolds equation and the JFO (Jakobsson and Floberg and Olsson) boundary conditions for non-Newtonian fluids, temperature effects on textured surfaces are studied theoretically. They show that in presence of surface texturing, the average temperature of the lubricating film reduces. To optimize the surface texturing parameters in parallel bearings, Guzek et al. [32] solved the Reynolds equation numerically, considering mass-conserving cavitation and viscosity changes due to temperature change. They showed that the decrease in viscosity due to the temperature rise can reduce the load-carrying capacity. Therefore, cavity height ratios should be higher in order to have a similar load-carrying capacity to textured surfaces with a constant temperature assumption. Gu et al. [33], in a study of the performance of surface texturing under starved and mixed lubrication, they employed a thermal mixed lubrication model considering the oil supply. They found that the start-up conditions can affect the friction coefficient. Moreover, they showed that it is easier for the textured surface to form the hydrodynamic lubrication than it is for the smooth surface, which is helpful to separate the mixed lubricated contact surfaces, and thus less friction heat is generated at the start-up phase. In the work of Bijani et al. [34] the influence of surface texturing on mixed lubricated contacts, different texturing patterns and cavity shapes is studied, and a numerical model to predict the friction is proposed.

Although the starved lubrication influence on film thickness in different applications has been studied extensively in more recent times, not much work has been done on mixed lubrication under starved lubrication conditions, and in the case of friction in starved lubricated textured surfaces even fewer studies have been done. When the lubricant in contact is limited to a specific amount, a correction of the film thickness formula is necessary, so in this study a corrected film thickness is presented for textured surfaces under starved lubrication conditions.

In order to develop the starved lubrication model, the modified film thickness relation for starved contacts is solved by taking the limited input film thickness into account, then the corrected film thickness is combined with the deterministic contact model. In this article the consequences of the existence of starvation in lubricated contacts on friction is discussed.

During the past decades, several efforts were devoted to studying this mixed lubrication frictional behaviour [35-38]. Based on the contact model, mixed lubrication models can be divided in two types: statistical and deterministic contact models. In the statistical models, the parameters represent the random characteristics of surface roughness. A major shortcoming of this model is its inability to provide detailed information on local roughness, which has an influence on the mechanisms of lubrication and friction. Another approach to simulating the frictional behaviour of contacting asperities results in a deterministic model, which employs the deterministic information of surface roughness. In these models, by summing up the local components of load and contact area for a given separation, it is possible to calculate deterministically the real contact area and the total force carried by the contact.

In 1972 Johnson, Greenwood and Poon [39] developed a model in which the load carried by a contact in the mixed lubrication regime is shared between the asperity contact and the fluid film. In their model, they combined the well-known Greenwood and Williamson [40] theory of random rough surfaces in contact with the elasto-hydrodynamic lubrication theory. This model was extended in 1999 by Gelinck 
and Schipper [41] to calculate the Stribeck curve for line contacts. Shi and Salant [42] introduced a mixed lubrication model, considering the inter-asperity cavitation and surface shear deformation for soft materials, and showed the occurrence of local cavitation. For moderately-loaded lubricated systems, the Jakobsson-Floberg-Olsson [12,13] cavitation theory is used. In 1970, Greenwood and Tripp introduced a deterministic contact model between two identical rough surfaces.

The flow factor method was introduced by Patir and Cheng $[43,44]$. They solved the Reynolds equation on a small area of the rough lubricating gap. The calculated micro flow is related to the flow of a perfectly smooth lubricating gap with similar mean height, resulting in flow factors. Fluid flow is assumed to have two sources, shear driven flow and pressure. The flow factors are calculated independently by solving the local deterministic flow problem for a specified roughness topography. The main drawback of this method is that the roughness asperities are not identical to the coordinate axes; this method is not effective in modelling the cross-flow of anisotropic roughness. Hu et al. [45] present a numerical solution for the contact of elastic bodies with three-dimensional roughness. The elastic contact has been modelled as a linear complementarity problem and is solved by the Conjugate Gradient Method. Yu et al. [46,47] developed a full numerical solution to mixed lubrication in point contacts. They viewed the asperity contact as a result of a continuous decrease in the film thickness. By employing this assumption, the transition from contact to non-contact is continuous, and as a result, the same mathematical model should work for both regions. To calculate the asperity contact problem a multi-level integration method is used. In the work of Faraon et al. [48], the Stribeck curves were calculated by developing a numerical model for a real distribution of the asperities; based on this model they compared the Stribeck curves between the deterministic and statistical model. They showed that the Stribeck curve results obtained with the statistical and the deterministic contact models are significantly different when the distribution of the surface heights deviates from the Gaussian height distribution; by performing experimental measurements they then showed that the deterministic mixed lubrication model is in good agreement with the measurements.

Recent developments in texturing techniques made it possible to employ different geometrical microand meso-scale patterns on the surface. These surface modification techniques include machining, photoetching, etching techniques, ion beam texturing and laser texturing [49]. Laser surface texturing proves to be more efficient, accurate, convenient and controllable for many materials [50], and is used to study the effect of micro-scale cavities on the frictional behaviour of contacts [25,27,29,37,51-56]. Kovalchenko et al. [27] show the influence of texturing on the transitions between the different lubricating regimes. They show that LST is able to enhance the hydrodynamic lubrication regime and thus increase the load-carrying capacity of the contact. Moreover, they found that the lapping after laser texturing that is carried out to remove the bulges at the edges of dimples is essential for increasing the positive effect of LST. In another study, Ryk et al. [29] investigated theoretically and experimentally the beneficial effects of applying LST on piston rings.

They observed that the benefits of LST in both full and starved lubrication conditions results in fuel consumption reduction in combustion.

In the work of Bijani et al. [34] on the influence of surface texturing on mixed lubricated sliding contacts, the deterministic asperity contact model is applied. By employing this contact model and solving the Reynolds equation, Stribeck-like curves for several cavity patterns with different geometry were plotted, and the behaviour of the coefficient of friction based on these parameters was investigated.

\section{Materials and Methods}

Here a deterministic mixed lubrication model is developed; in this model, the lubrication is based on a limited amount of lubricant supplied to include the effect of starvation on friction in parallel sliding contacts. This is realized by combining a model calculating the film thickness in a sliding contact under starved conditions [57], with a deterministic asperity contact model to calculate and study the friction. The results are presented in Stribeck-like curves. By using this model, the frictional performance of starved lubricated contacts as a function of velocity and texturing parameters such as cavity depth, size and density will be analysed, in particular within hydrodynamic and mixed lubrication regimes under starved conditions.

\subsection{Deterministic Asperity Model}

The Greenwood and Williamson contact model [40] assumes that asperities are spheres with a similar radius, and that asperity heights can vary randomly with a Gaussian probability distribution. However, in reality, this is rarely the case, as all of the asperities have the same radius; also, representing them as 
spheres or ellipsoids is more accurate. Moreover, the Gaussian height distribution is not an accurate approximation for most of the surfaces. As a recent advancement in optical measurement tools, the interference microscope can provide more accurate digital data of the surface topography which could be applicable for different applications, such as the calculation of the load carried by the deformed asperities of a rough surface when it is in contact with a flat surface (see Figure 1).

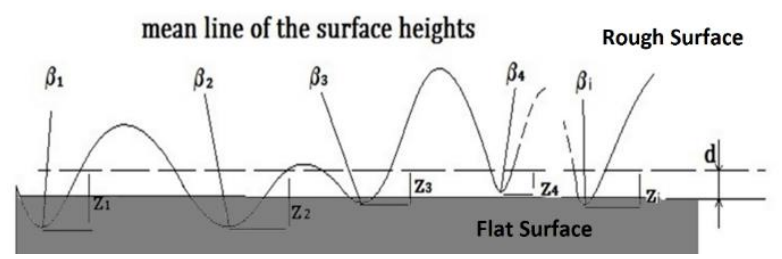

Figure 1. The schematic illustration of a flat surface and rough surface contact [34].

Figure 1, shows a flat on rough surface contact. For a given separation of two surfaces $(d)$, the total force carried by the contact, the real contact area and the number of asperities $s$, are measurable deterministically by summing the local contributions of the above-mentioned parameters. The asperities are assumed as ellipsoids with different radii $\left(\beta_{x i}\right.$ are the asperity radii in the sliding direction, and $\beta_{y i}$ is in perpendicular direction), as well as the fact that they deform independently of each other. From Figure 2, the deformation of an asperity can be defined as Equation 1:

$$
w_{i}=z_{i}-d
$$

where $z_{i}$ is the individual summit height, and $w_{i}$ is the indentation of each deformed asperity.

For a given value of $w_{i}$, by adding the individual components of each asperity contact, the asperities' normal load $\left(F_{C}\right)$ and the real contact area can be determined.

\subsection{Load Sharing Concept}

According to Johnson [39], in the case of a mixed lubrication regime $(\mathrm{ML})$, the total normal load $\left(F_{T}\right)$ is equal to the load carried by the boundary lubrication $\mathrm{BL}$ force component $\left(F_{C}\right)$ plus the hydrodynamic lubrication $\mathrm{HL}$ force component $\left(F_{H}\right)$, therefore:

$$
F_{T}=F_{C}+F_{H}
$$

Based on (Equation 2), coefficients $\left(\gamma_{1}\right)$ and $\left(\gamma_{2}\right)$ are introduced:

$$
\gamma_{1}=\frac{F_{T}}{F_{H}}, \gamma_{2}=\frac{F_{T}}{F_{C}}
$$

The two coefficients $\left(\gamma_{1}\right.$ and $\left.\gamma_{2}\right)$ are dependent on each other through the equation:

$$
1=\frac{1}{\gamma_{1}}+\frac{1}{\gamma_{2}}
$$

In the deterministic asperity contact model, the Stribeck curve for the contact between a rigid flat surface against a rough surface, can be calculated by employing the two $\left(\gamma_{1}\right)$ and $\left(\gamma_{2}\right)$ parameters, where these two coefficients are defined in Equation 3 . In the work of Gelinck [41], these two parameters are presented in terms of pressure (see Equation 5). By combining the well-known Greenwood and Williamson [40] contact model under a classical hypothesis of the Reynolds isothermal equation, the entire Stribeck curve can be calculated.

$$
\gamma_{1}=\frac{p_{T}}{p_{H}}, \gamma_{2}=\frac{P_{T}}{p_{C}}
$$

where $p_{T}$ is the total pressure carried by the contact, $p_{C}$ is the pressure on asperities, and $p_{H}$ is the pressure carried by the hydrodynamic component of mixed lubrication [48].

In mixed lubricated contacts, in order to calculate the coefficient of friction the asperity and hydrodynamic load components $\left(F_{C}\right.$ and $\left.F_{H}\right)$, as well as the related film thickness, must be determined. By solving the following three equations, it is possible to determine the above-mentioned three parameters:

I. The first equation is Equation $2\left(F_{T}=F_{C}+F_{H}\right)$, in order to consider the load components in the $\mathrm{BL}$ component and the $\mathrm{HL}$ component, and their relation to the total load.

II. The second equation is the film thickness relation. In this study, the film thickness calculation is based on solving the Reynolds equation for textured surfaces [57].

This Reynolds equation is derived from the NavierStokes equation by taking the narrow gap assumption into account. In the Cartesian coordinate system, the Reynolds equation can be written as:

$$
\begin{aligned}
\frac{\partial}{\partial x}\left(\frac{\rho h^{3}}{\eta}\right. & \left.\frac{\partial p}{\partial x}\right)+\frac{\partial}{\partial y}\left(\frac{\rho h^{3}}{\eta} \frac{\partial p}{\partial y}\right) \\
& =6\left(u_{0}\right) \frac{\partial(\rho h)}{\partial x} \\
& +6 \rho h \frac{\partial\left(u_{0}\right)}{\partial x}+12 \frac{\partial(\rho h)}{\partial t}
\end{aligned}
$$

where $p$ is the lubricant pressure, $\eta$ is the viscosity, $h$ is the film thickness, and $u_{0}$ is the sum velocity. In the absence of textures over the surface in parallel sliding contacts, the right side of the Reynolds equation equals zero; therefore there will be no pressure build-up in contact, and no film can get formed.

At the outlet of the cavity, the lubricant is dragged through a converging region, and as a result, pressure 
is generated. The flow divergence at the entry of the cavity results in negative pressure. This negative pressure is suppressed by cavitation, and as a cavitation product, vapour bubbles appear in the lubricant film. The Jakobsson-Floberg-Olsson model divides the lubrication film into two zones. The first zone is the lubricant film without the cavitation effect; therefore no vapour bubble exists in this zone, and the Reynolds equation is valid. In the second zone, where cavitation does take place, the lubricant occupies just a fraction of the film gap, and the vapour bubbles exist in the void fraction. In this zone the pressure is taken as a constant, [58].

By suggesting the use of a switch function, Elrod introduced a universal solution for cavitated and full film zones (see Equation 7). In equation 7, $\varphi$ represents a dimensionless dependent variable and $F$ is the aforementioned switch function, and these parameters are defined as in a liquid zone, where $F=1, \varphi \geq 0$ and $p=\varphi$, and in the cavitated region, $F=0, \varphi<0$ and $p=0$.

The steady-state mass-conservation Reynolds equation, taking the Elrod cavitation algorithm into account, can be written in a Cartesian coordinate system as (Equation 7) [59]:

$$
\begin{aligned}
& \frac{\partial}{\partial x}\left(\frac{h^{3}}{\eta} \frac{\partial(F \varphi)}{\partial x}\right)+\frac{\partial}{\partial x}\left(\frac{h^{3}}{\eta} \frac{\partial(F \varphi)}{\partial y}\right) \\
= & \frac{6 u_{0}}{p_{a}-p_{c}} \frac{\partial((1+(1-F) \varphi) h)}{\partial x}
\end{aligned}
$$

In this study, chevron and groove patterns have been investigated. Figure 2, shows the different cavity shapes and the parameters characterizing their geometry. The chevron pattern is defined by two similar equilateral triangles of different sizes. When the inner edge length of the chevron approaches zero, the chevron pattern transforms into a triangular pocket. For these two cases, the centre of the unit cell coincides with the midpoint of the altitude line of the triangle or chevron shape, (see also [60]). All patterns have a rectangular cross-sectional profile, (see Figure 3 ). The general film thickness formula can be written as (Equation 8):

$$
h=h_{0}+h_{\text {macro }}+h_{\text {texture }}
$$

When both interacting surfaces of contact are flat, $h_{\text {macro }}$ is negligible. In the case of a flat on flat sliding contact, the film thickness (Equation 8) reduces to (Equation 9) $[60,61]$ :

$$
\frac{h(x, y)}{h_{0}(x, y)}=1+H(x, y)
$$

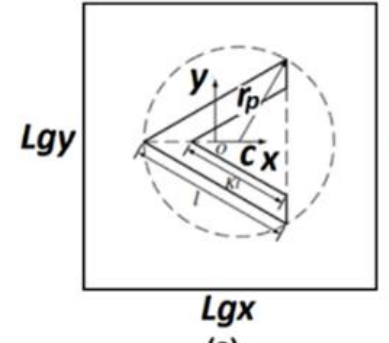

(a)

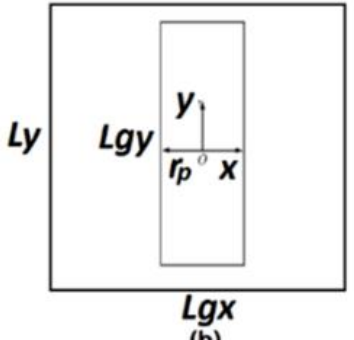

(b)
Figure 2. Geometrical scheme of patterns: (a)

$$
\text { Chevron, (b) Groove }
$$

(Reproduced with the permission of Mingfeng

Qiu, Bret R. Minson, Bart Raeymaekers, Tribology International, published by Elsevier, 2013) [60]w.

The film thickness formula for the chevron can be written as (Equation 10):

$$
\begin{array}{r}
H(x, y)= \begin{cases}0, & (X, Y) \notin \Omega \\
\frac{T_{d}}{h_{0}}, \quad & (X, Y) \in \Omega\end{cases} \\
\Omega:-\frac{3}{4} \leq X \\
\leq \text { and }\left\{\begin{array}{c}
\frac{1}{\sqrt{3}} X+\frac{\sqrt{3}}{2}\left(K-\frac{1}{2}\right) \leq \\
Y \leq \frac{1}{\sqrt{3}} X+\frac{\sqrt{3}}{4} \\
-\frac{1}{\sqrt{3}} X-\frac{\sqrt{3}}{4} \leq \\
Y \leq-\frac{1}{\sqrt{3}} X+\frac{\sqrt{3}}{2}\left(\frac{1}{2}-K\right)
\end{array}\right.
\end{array}
$$

The film thickness formula for the grooves is given in (Equation 11):

$$
\Omega:-\frac{1}{2} \leq X \leq \frac{1}{2} \text { and } \frac{1}{2} \leq Y \leq \frac{1}{2}
$$

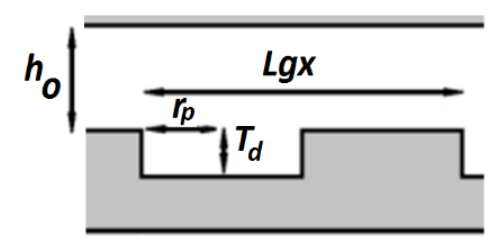

Figure 3. Schematic illustration of the cavity profile.

In this simulation $r_{p}$ is the characteristic radius for the chevron patterns and the half width of the grooves.

To solve the Elrod cavitation algorithm for Reynolds equation (Equation 7) the tri-diagonal matrix algorithm (TMDA) is used, and in order to reduce the storage needed for calculation the line-by-line TDMA solver (Patankar [62]) is employed. The TDMA is a direct method for a one-dimensional situation, but by solving it iteratively line-by-line it is possible to apply it for two 
and three-dimensional problems as well. [63]. The algorithm for this numerical solution is presented in appendix $A$.

III. The third equation is derived from the equilibrium of the modified relation for the central pressure and average contact pressure carried by asperities, represented as [48].

$$
F_{\mathrm{C}}=\sum_{i=1}^{N} \frac{2}{3} E^{\prime} R_{i}\left(z_{i}-d\right)
$$

In Equation 12, $E^{\prime}$ is the combined elasticity modulus and $R_{i}$ is the reduced radius of the cylinder. The reduced elastic modulus is given by:

$$
\frac{2}{E^{\prime}}=\frac{1-v_{1}^{2}}{E_{1}}+\frac{1-v_{2}^{2}}{E_{2}}
$$

where $E_{1}=E_{2}=E$ and $v_{1}=v_{2}=v$.

The total friction force $\left(F_{f}\right)$ in the $\mathrm{ML}$ regime can be calculated as the sum of the shear force of the lubricant $\left(F_{f H}\right)$ and friction force of the contacting asperities and (Equation 14):

$$
F_{f}=\sum_{i=1}^{N} \iint_{A_{c i}} \tau_{c i} d A_{c i}+F_{f H}
$$

In Equation 14, $\tau_{c i}$ is the shear stress at the asperity contact, $N$ is the number of contacting asperities and $A_{c i}$ the area of contact of a single asperity contact. For the hydrodynamic component of shear force $\left(F_{f H}\right)$, the friction force can be written as:

$$
F_{f H}=\tau_{H} A_{H}
$$

where $\tau_{H}$ is the shear stress of the lubricant and $A_{H}$ the contact area of the hydrodynamic component. Friction is assumed to be of the Coulomb type for the contacting asperities:

$$
f_{C i}=\frac{\tau_{C i}}{p_{C i}}
$$

with $p_{C i}$ the average contact pressure on the $i^{\text {th }}$ asperity, and $f_{C i}$ the coefficient of friction which is assumed constant for all asperities; then the double integral in Equation 14 can be written as:

$$
\sum_{i=1}^{N} \iint_{A_{c i}} f_{C} p_{c i} d A_{c i}=f_{C} F_{C}
$$

The value of $f_{C}$ is measurable from experiments, and in these calculations this value is set to 0.1 based on the measurements presented in appendix $B$. The coefficient of friction is given by:

$$
f=\frac{F_{f}}{F_{N}}=\frac{f_{C} F_{C}+F_{f H}}{F_{N}}
$$

It is worth mentioning that, in the absence of texturing features in parallel sliding, no lubricant film in contact can be formed, and the two sliding surfaces will stick to each other. Therefore, the coefficient of friction will be constant and equal to the coefficient of friction in the boundary lubrication regime $\left(f_{C}\right)$.
In order to study the effect of starvation for textured surfaces with different texturing patterns, and to investigate the frictional behaviour based on the different texturing parameters, several simulations are carried out.

\section{Problem Definition and Its Solution}

In order to investigate the effect of lubricant supply on the coefficient of friction for different texturing parameters and patterns, several simulations were performed.

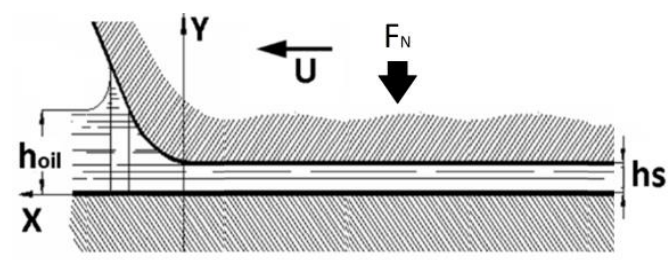

Figure 4. Schematic illustration of the input film thickness $\left(h_{\text {oil }}\right)$ and the calculated film thickness $\left(h_{s}\right)[64]$.

In Figure 4, when the input film thickness $\left(h_{\text {oil }}\right)$ is smaller than the calculated film thickness for nonstarved lubrication $\left(h_{s}\right)$, the contact is operated under starved conditions. The limited amount of lubricant in the input region of contact $\left(h_{\text {oil }}\right)$ can affect the coefficient of friction for the textured surfaces. To gain a better understanding of the starved lubrication phenomenon, the influence of input film thickness and texturing parameters, such as texture pitch $\left(P_{x}\right)$, texture depth $\left(T_{d}\right)$ and texture size $(S)$, on the coefficient of friction is investigated. These simulations are based on the linear groove and chevron patterns (see Figure 5).

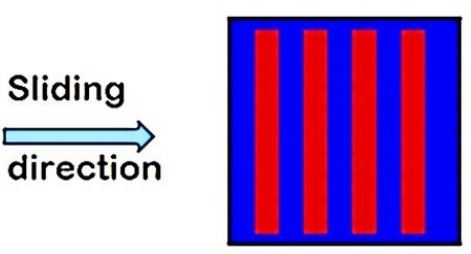

(a)

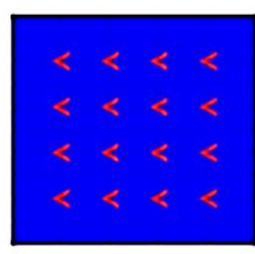

(b)
Figure 5. Schematic illustration of different patterns (a) Grooves, (b) Chevron pattern [34].

In order to investigate the effect of texturing, the geometrical parameters are studied. In this parametric study, the effect of the geometrical parameters determining the texture shape and the effect of the textured area fraction upon the frictional behaviour of the starved lubrication conditions are studied. By introducing texture pitch $\left(P_{x}\right)$, it is possible to define the textured area fraction. 
The pitch is calculated as:

Cavity size: $S=2 r_{p}$ and Pitch in $\mathrm{x}$ direction: $P_{x}=$ $S / L_{g x}$

In addition, texture depth $\left(T_{d}\right)$ can affect the film formation in friction in textured surfaces. It is possible to define the geometry of grooves by these three parameters. Another parameter that can help to define the geometry of chevrons is the cavity width ratio, which is represented by $K$ in Figure 6 .

Prior study of film thickness [57] showed that the rectangular cross-section can build a thicker lubricant film in contact and is more efficient than the circular cross-sectional patterns; therefore in the present article the rectangular cross-section patterns are employed (see Figure 3). Here, linear grooves and chevrons will be analysed. The operating conditions applied in this calculation are presented in Table 1. The analyses of the roughness measurement and surface topography were performed using a Keyence Color 3D LASER Scanning Microscope (Keyence, Osaka, Japan), which uses a violet LASER $\lambda=388 \mathrm{~nm}$.

Table 1. Operating conditions.

\begin{tabular}{cc}
\hline Properties & Value \\
\hline Normal load & $5 \mathrm{~N}$ \\
Average contact pressure & $0.05 \mathrm{MPa}$ \\
Contact area & $10^{-4} \mathrm{~m}^{2}$ \\
Lubricant viscosity & $8 \mathrm{mPa} \cdot \mathrm{s}$ \\
\hline
\end{tabular}

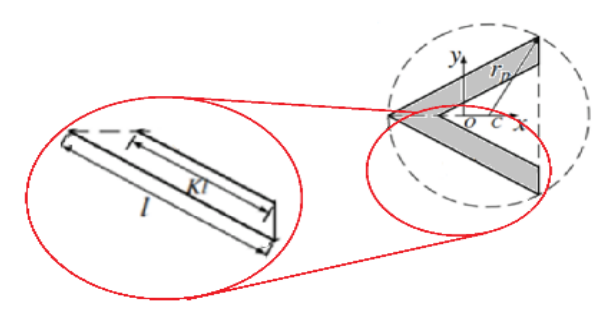

Figure 6. Chevron cavity width ratio.

where $K=$ Inner wall length/outer wall length. In these calculations $(K)$ is constant, and equal to 0.5 .

To validate the model and algorithm, a comparison was performed between the experimental measurements of Kovalchenko et al. [37] and the numerical results from the algorithm developed in this work. It is worth mentioning that, although the developed algorithm can calculate the friction in all three lubrication regimes within this section just to validate the code, the comparison takes place in the hydrodynamic lubrication regime. Therefore, the numerical and measurement results are dealing only with the hydrodynamic lubrication regime. Kovalchenko et al. [37] investigated the effect of size and the density of circular pockets on the coefficient of friction (see Figure 7). In this study the Disk 3 results are compared with results from the developed numerical algorithm.

Disk 3

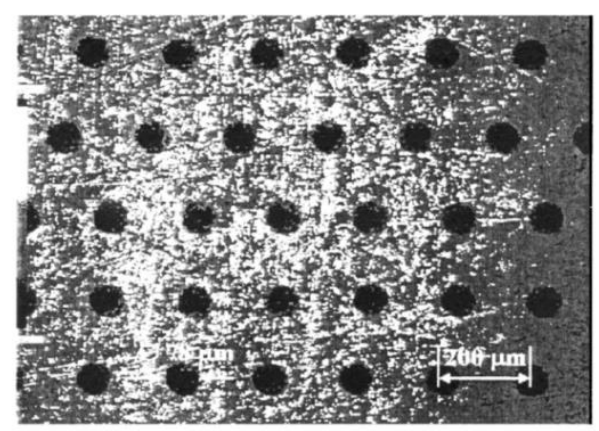

Figure 7. Disk 3 dimple array, (Reproduced with permission from Andriy Kovalchenko, Oyelayo Ajayi, Ali Erdemir, et al., Tribology Transactions, published by Taylor and Francis, 2004) [37].

In Disk 3 the cavity depth is $5.5 \mu \mathrm{m}$, the cavity size $\left(S=2 r_{p}\right)$ is $78 \mu \mathrm{m}$ and the texture density is $28 \%$. The measurement results for this disk are presented in Figure 8.

Experimental data extracted from Figure 8 and the numerical results are based on the calculation of coefficient of friction for the full film condition, when the lubricant kinematic viscosity is $1247 \mathrm{cSt}$ at $40{ }^{\circ} \mathrm{C}$, and the normal load of $20 \mathrm{~N}$ is applied.

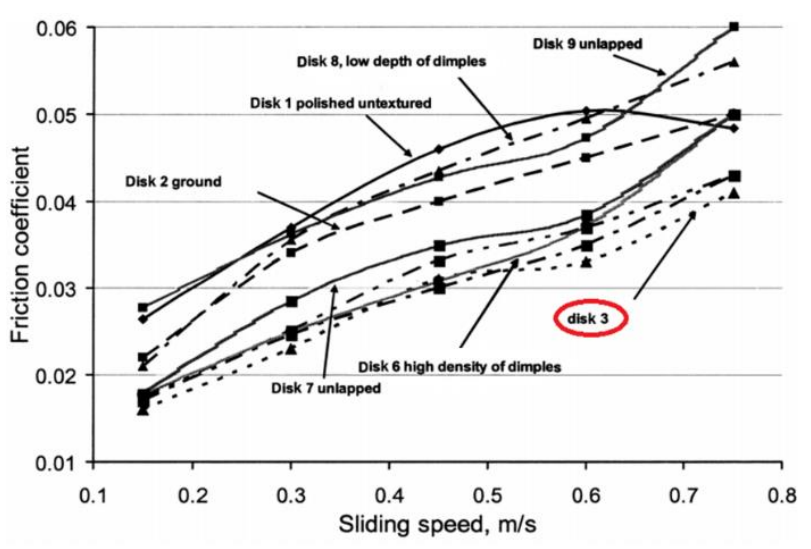

Figure 8. Measurement results (Reproduced with permission from Andriy Kovalchenko, Oyelayo Ajayi, Ali Erdemir, et al., Tribology Transactions, published by Taylor and Francis, 2004) [37]. 


\section{- 00 \\ - 00 \\ - 00}

(a)

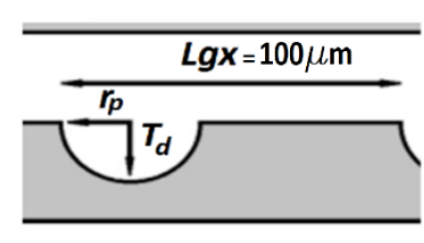

(b)
Figure 9. (a) Simulated texture array and (b) cavity profile.

The texture array is illustrated schematically in Figure 9. The comparison between the numerical results and the experimental measurements for circular pockets with $T_{d}=5 \mu \mathrm{m}, r_{p}=15 \mu \mathrm{m}$ and $P_{x}=0.3$, are presented in Figure 10.

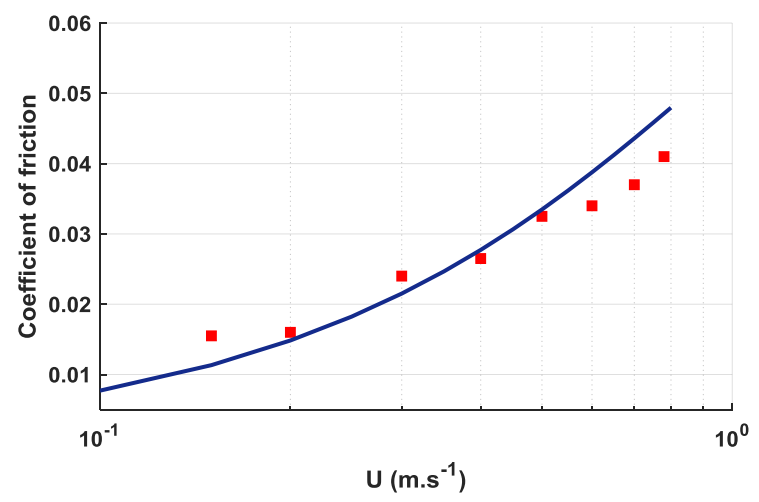

Figure 10. Comparison between numerical and experimental results.

From Figure, 10 it is possible to see that there is good agreement between the values of the coefficient of friction calculated by the numerical model (blue line) and the experimentally measured results (red diamonds). When the velocity is $0.5 \mathrm{~m} \cdot \mathrm{s}^{-1}$ the calculated value is around $1 \%$ less than the measured value for Disk 3 that is in reasonable range. Moreover, results show that the calculated coefficient of friction has the same trend as the measured results for the coefficient of friction.

\section{Results}

\subsection{The Effect of Texture Depth $\left(T_{d}\right)$}

In order to study the influence of the effect of texturing in starved lubricated contact with respect to the texture depth, several simulations were carried out, based on groove and chevron texturing patterns. In these calculations, the texturing parameters other than the texture depth are constant. The coefficient of friction is calculated based on three different values of texture depth, and ranges from 5 to $10 \mu \mathrm{m}$. In Figure 11 and Figure 12, the simulation results show the effect of different values for the texture depth based on starved and non-starved lubrication for grooves and chevrons. In Figure 11a, it can be seen from the results that a depth of $10 \mu \mathrm{m}$ gives the lowest coefficient of friction. In Figure 11b, a limited input film thickness is applied in order to simulate the starvation. The results in Figure $11 \mathrm{~b}$ show that the calculated coefficient of friction for different values of texture depth tends to the same values, therefore the Stribeck curves are merging together. In the calculations shown, the input film thickness $\left(h_{\text {oil }}\right)$ is set to $10 \mu \mathrm{m}$. The texturing properties of subsequent calculations are given in Table 2.

Table 2. Texturing properties.

\begin{tabular}{cc}
\hline Parameter & Value \\
\hline Texture pitch $\left(P_{x}\right)$ & 0.4 \\
Cavity size $(S)$ & $100 \mu \mathrm{m}$ \\
\hline
\end{tabular}

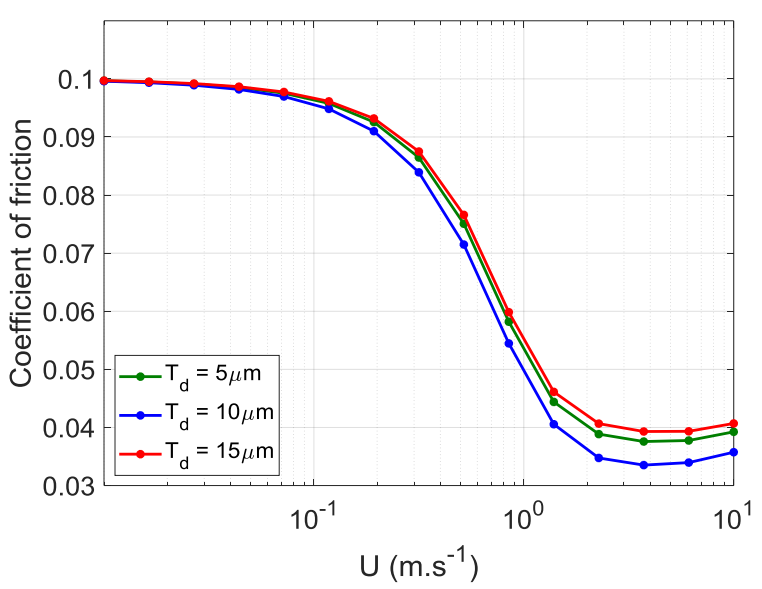

(a)

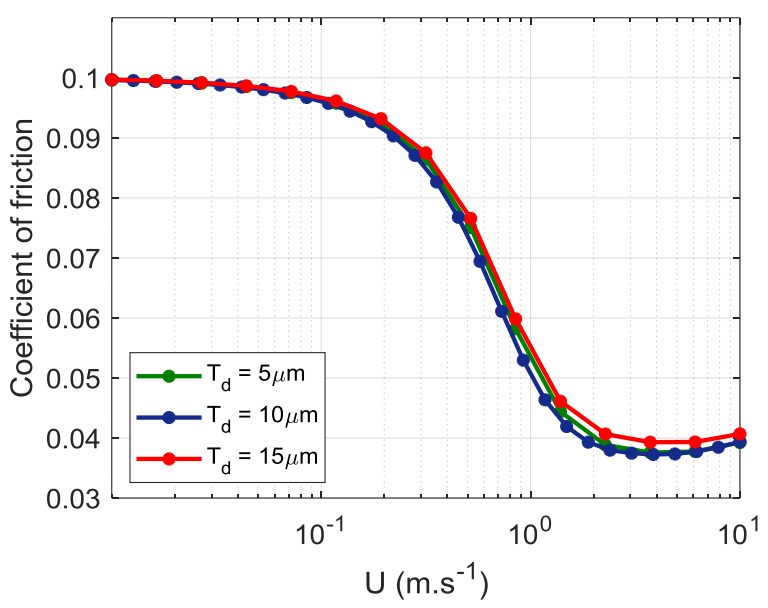

(b)

Figure 11. Stribeck curves as a function of texture depth for grooves when the lubrication is: (a) Non-starved, (b) starved, and $h_{\text {oil }}=$ $10 \mu \mathrm{m}$. 


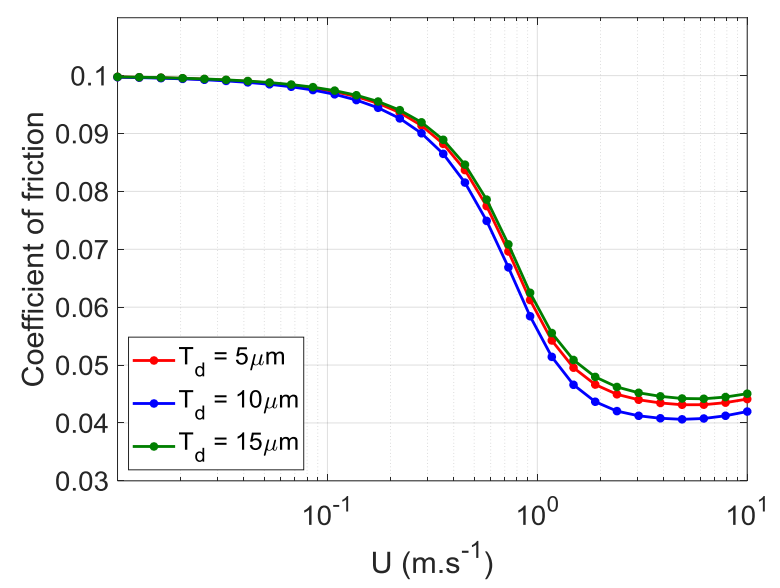

(a)

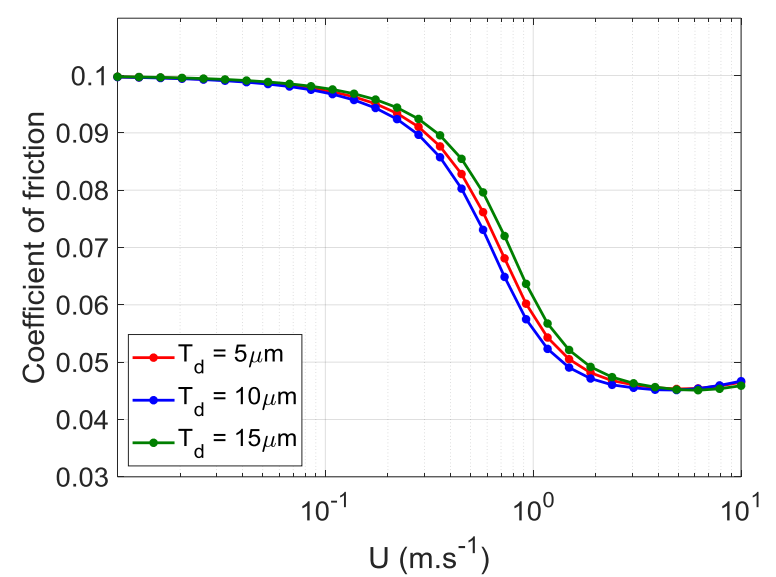

(b)

Figure 12. Stribeck curves as a function of texture depth for chevrons when the lubrication is: (a) Non-starved, (b) starved, and $h_{\text {oil }}=$ $10 \mu \mathrm{m}$.

In Figure 12a, in the case of chevron patterns similar to the groove pattern, the lowest coefficient of friction in the case of non-starved lubrication is achievable when the depth is around $10 \mu \mathrm{m}$. In Figure $12 \mathrm{~b}$, in the case of starved lubrication, results show that the calculated coefficient of friction for different values of texture depth is tending to the same values, therefore the Stribeck curves are merging. By comparing the curves in Figure 12, and based on the results from the study on film thickness [57] (which showed that a greater film thickness is achievable for grooves and chevrons when the depth is around $10 \mu \mathrm{m})$, it is possible to conclude that the lowest coefficient of friction is achievable when the film thickness has the highest value. This is because in the case of mixed lubrication an increasing film thickness separates the surfaces and reduces contact. Although the growth in the depth of cavities to the optimum values leads to the greater film thicknesses, in the case of starved lubrication, this growth in lubricant film thickness is limited due to the limit in oil supply; this will result in a limited effect of this parameter on the coefficient of friction as shown in Figure 12.

\subsection{The Effect of Texture Size (S)}

In this section, to study the effect of size on the coefficient of friction for starved lubricated contacts, the texture depth and pitch are set as constant, and the size is changed. The coefficient of friction is calculated for three different texture size parameters $(S)$,. In Figure 13 and Figure 14, the simulation results show the effect of different values for texture size-based-starved and non-starved lubrication for grooves and chevrons. In Figure 13a, in the case of groove patterns when the size is around $100 \mu \mathrm{m}$, the friction force has the lowest value. In Figure 13b, the limited input film thickness is applied in order to simulate the starvation. The results in Figure 13b show that the calculated Stribeck curves for different values of texture size are merging in the case of starved lubrication. Texturing properties for calculations of Figure 13 and Fig 14 are shown in Table 3.

Table 3. Texturing properties.

\begin{tabular}{cc}
\hline Parameter & Value \\
\hline Texture depth $\left(T_{d}\right)$ & $10 \mu \mathrm{m}$ \\
Texture pitch $\left(P_{x}\right)$ & 0.4 \\
\hline
\end{tabular}

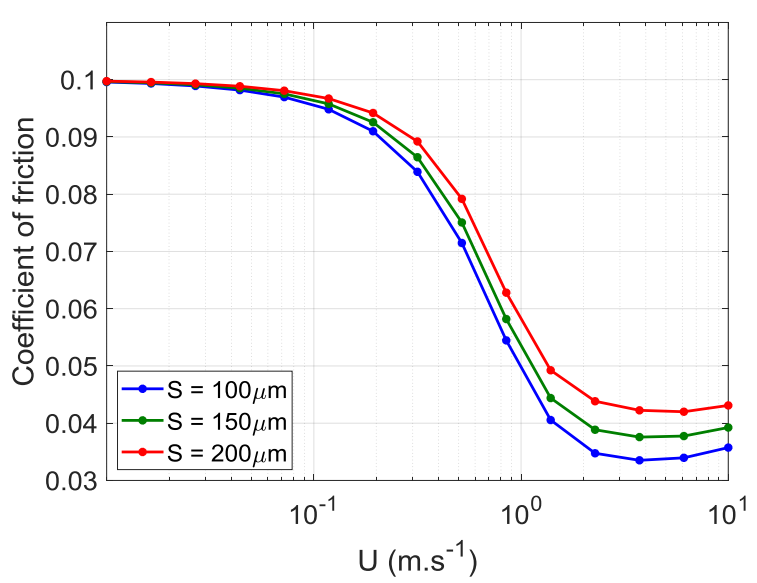

(a) 


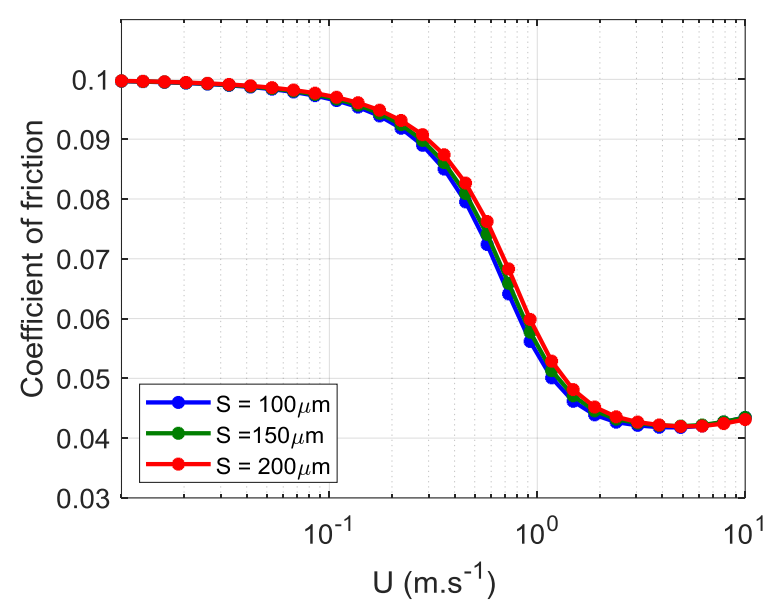

(b)

Figure 13. Stribeck curves as a function of cavity size $(S)$ for grooves when the lubrication is: (a) Non-starved, (b) starved, and $h_{\text {oil }}=10 \mu \mathrm{m}$.

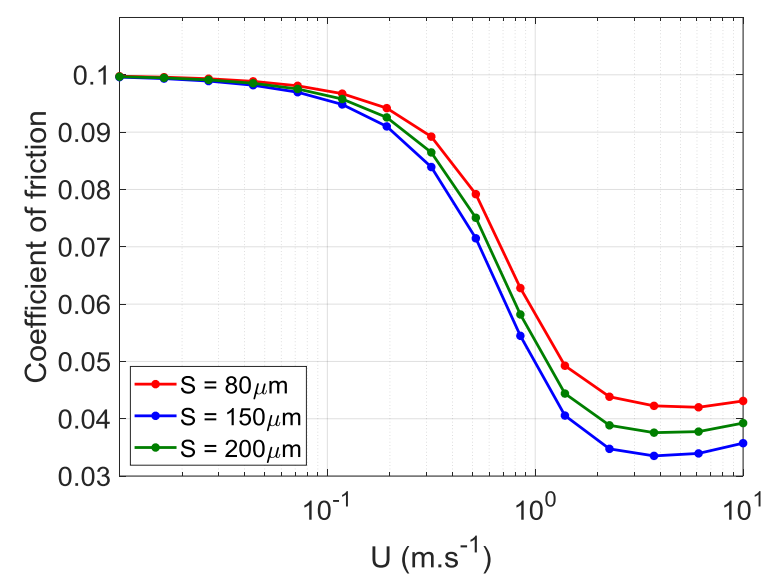

(a)

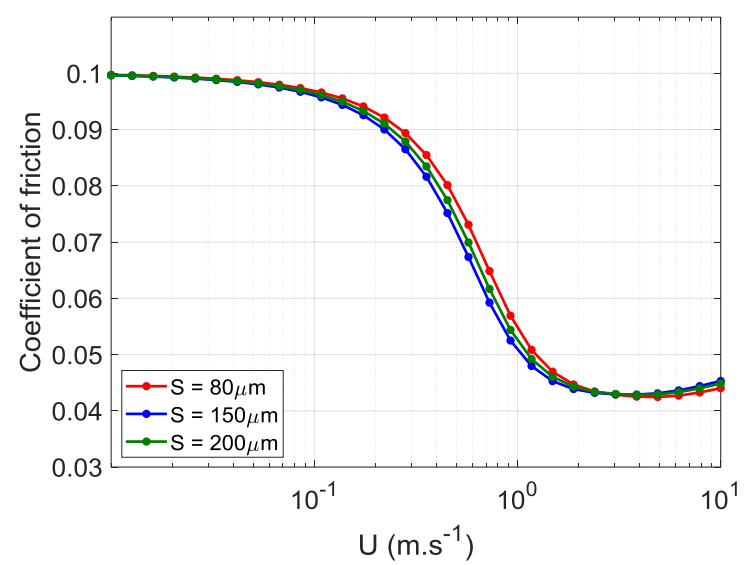

(b)

Figure 14. Stribeck curves as a function of cavity size for chevrons when the lubrication is: (a) Non-starved, (b) starved, and $h_{\text {oil }}=10 \mu \mathrm{m}$.

In Figure 14a, in the case of a chevron pattern similar to the groove pattern, the lowest coefficient of friction in the case of non-starved lubrication is achievable when the size is around $150 \mu \mathrm{m}$. Also in this case, starved lubrication results in the merging of the curves as shown in Figure 14b. Consequently, as mentioned and as achieved and shown in Figure 14, changing the size parameters has an influence on the coefficient of friction, but this effect is limited with respect to values close to the optimum value for this parameter.

\subsection{The Effect of Texture Pitch $\left(P_{x}\right)$}

In Figure 15 and Figure 16, the simulation results show the effect of different values for texture pitch based on starved and non-starved lubrication for grooves and chevrons. In Figure 15a, in the case of a groove pattern when the pitch is around 0.4 , according to the results from the study on film thickness [57] the lubricant film is thicker; when the lubrication is in a mixed lubrication regime the thicker film results in a lower solid-solid contact. Therefore, the friction has the lowest value when $P_{x}=0.4$. In Figure 15b, the limited input film thickness is applied in order to calculate the friction in the starved lubrication. The results in Figure $15 \mathrm{~b}$ show that when the lubrication is in the starved regime the calculated coefficient of friction for different values of texture size is tending to the same values, therefore the Stribeck curves are merging together. Texturing properties for calculations of Figure 15 and Figure 16 are shown in Table 4.

Table 4. Texturing properties

\begin{tabular}{cc}
\hline Parameter & Value \\
\hline Texture depth $\left(\mathrm{T}_{\mathrm{d}}\right)$ & $10 \mu \mathrm{m}$ \\
Cavity size $(\mathrm{S})$ & $100 \mu \mathrm{m}$ \\
\hline
\end{tabular}

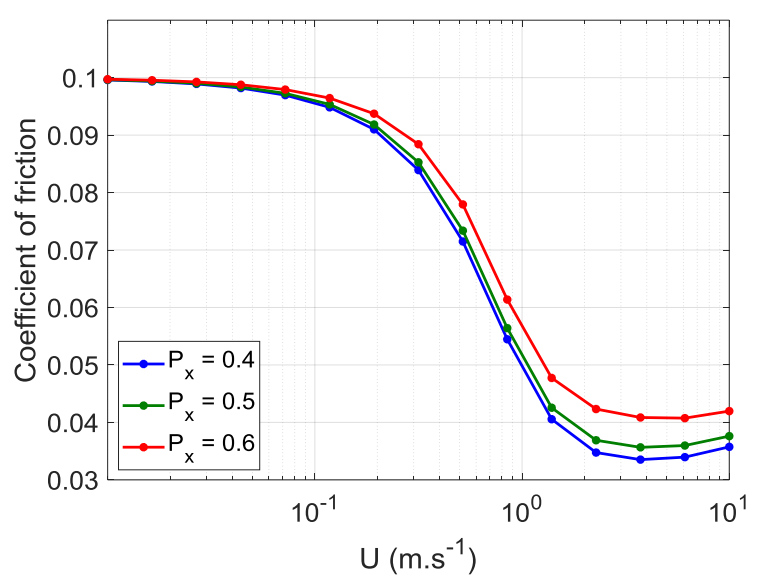

(a) 


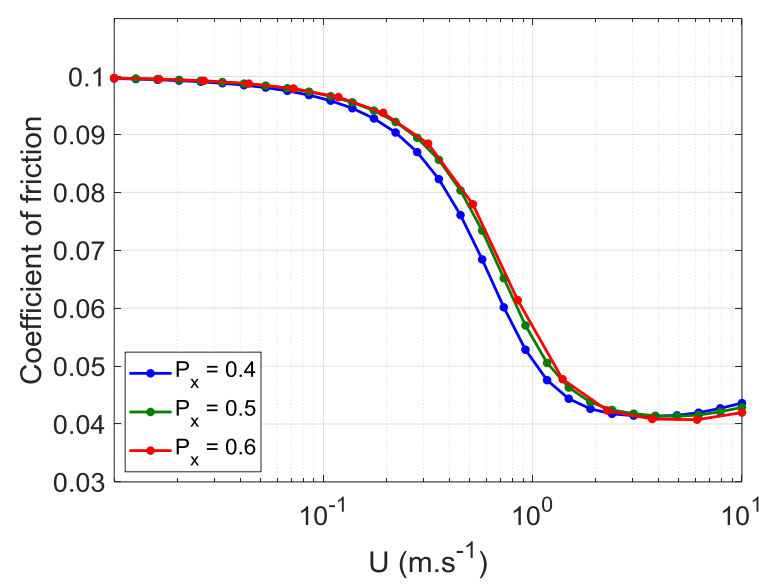

(b)

Figure 15. Stribeck curves as a function of texture pitch for grooves when the lubrication is:

(a) Non-starved, (b) starved, and $h_{\text {oil }}=10 \mu \mathrm{m}$.

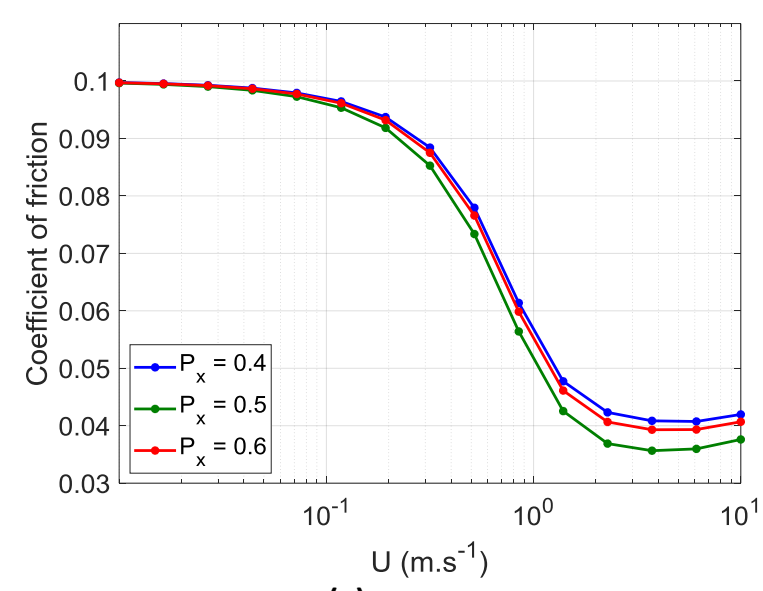

(a)

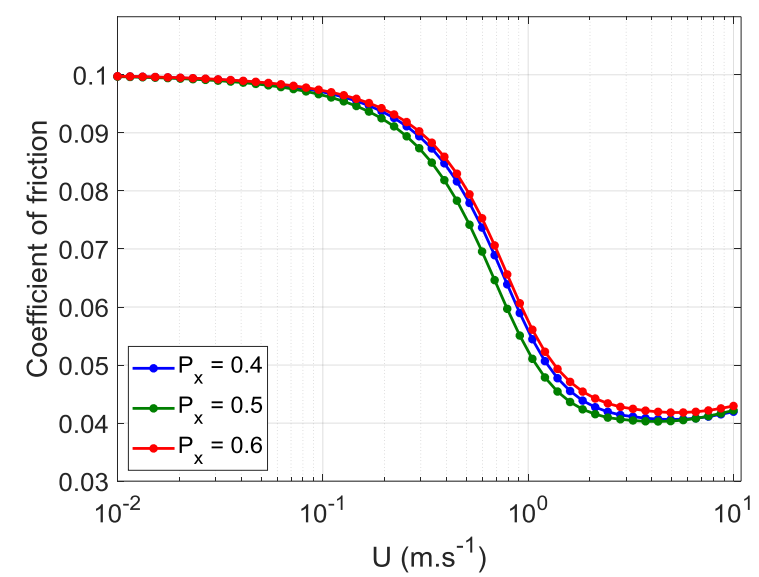

(b) friction in the case of non-starved lubrication is achievable when the pitch is around 0.5 . In Figure $16 \mathrm{~b}$, it is possible to observe the same behaviour of the merging in Stribeck curves for chevrons in Figure 12b, for the groove pattern. Therefore, due to the limit in lubricant supply, which results in limited lubricant film growth, the same trend of behaviour is also predictable for other patterns when the starvation in lubrication occurs, i.e. triangular pockets and circular pockets.

\subsection{Effect of Input Film Thickness on the Coefficient of Friction}

In Fig. 17.a, the calculated film thickness and in Fig. 17.b, the calculated coefficient of friction for grooves, when $T_{d}=10 \mu m, S=100 \mu m$ and $P_{x}=0.4$, is shown. In these calculations, the effect of different input film thickness is studied. The coefficient of friction for four different input film thicknesses $h_{\text {oil }}=$ $0.025,4,6$ and $8 \mu m$ and the film thickness for three different input film thicknesses $h_{\text {oil }}=0.5,4$ and $6 \mu \mathrm{m}$ are calculated.

Table 5. Texturing properties and operating conditions.

\begin{tabular}{cc}
\hline Parameter & Value \\
\hline Texture depth $\left(\mathrm{T}_{\mathrm{d}}\right)$ & $10 \mu \mathrm{m}$ \\
Texture pitch $\left(\mathrm{P}_{\mathrm{x}}\right)$ & 0.4 \\
Cavity size $(\mathrm{S})$ & $100 \mu \mathrm{m}$ \\
Normal load $\left(\mathrm{F}_{\mathrm{T}}\right)$ & $5 \mathrm{~N}$ \\
Average contact pressure & $0.05 \mathrm{MPa}$ \\
Lubricant viscosity & $0.08 \mathrm{~Pa} \cdot \mathrm{s}$ \\
\hline
\end{tabular}

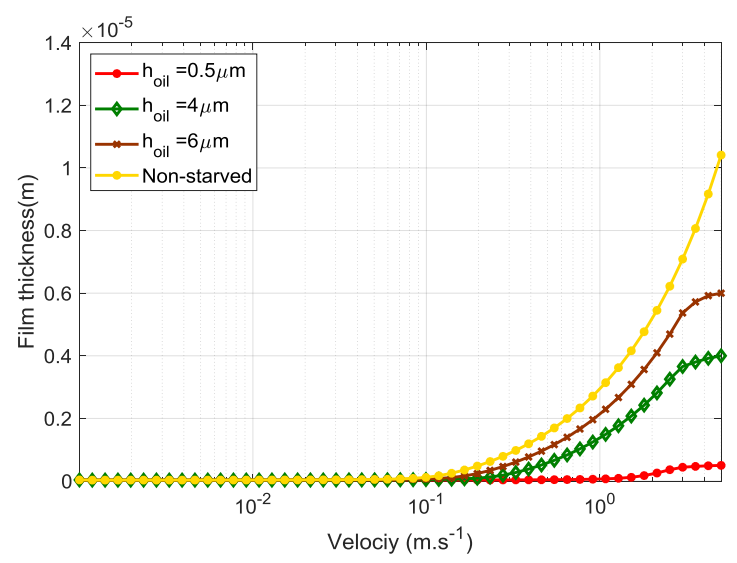

(a)

Figure 16. Stribeck curves as a function of texture pitch for chevrons when the lubrication is: (a) Non-starved, (b) starved, and $h_{\text {oil }}=$ $10 \mu \mathrm{m}$.

In Figure 16a, in the case of a chevron pattern similar to the groove pattern, the lowest coefficient of 


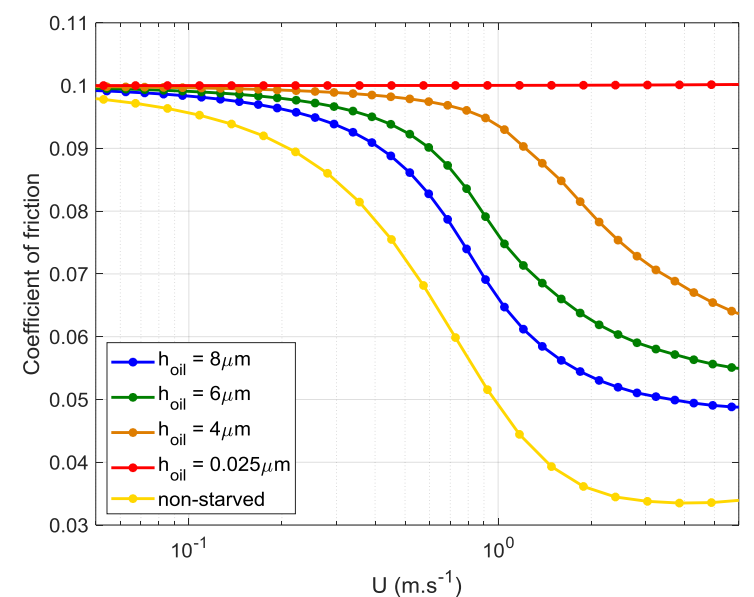

(b)

Figure 17. Effect of input film thickness on (a) film thickness, (b) coefficient of friction.

The results of the Stribeck curve and the film thickness for different values of the oil thickness applied to the contact are presented in Fig. 17.a and Fig. 17.b. The behaviour of the starved Stribeck curve and the film thickness as a function of the applied oil thickness can be described as follows:

1. When the input film thickness $\left(h_{\text {oil }}\right)$ is lower than $0.025 \mu \mathrm{m}$, the Stribeck curve transforms into a straight line, and the film thickness for different velocities stays constant in the same level as it is in the BL regime.

2. If the $h_{\text {oil }}$ varies between 0.025 and $8 \mu \mathrm{m}$, the friction level in the $H L$ and $M L$ regimes starts to decrease, and the film thickness increases. By reducing the value of applied oil layer thickness to the contact, the mixed lubrication regime becomes less steep in this range of applied oil film thickness.

It concludes that, although the starvation has no influence on the transition of lubrication regimes, the friction level changes due to starved lubrication.

\section{Discussion}

In this article, the influence of starvation and surface texturing on each other has been investigated by performing a set of parametric studies. The variation range of texturing parameters in these parametric studies was chosen based on the sizes which can give more fluctuation in the frictional behaviour of contacts; these ranges were chosen from the previous study on the effect of surface texturing on film thickness [57].
Based on the results in this section, texturing for flat-flat sliding contacts with a starved lubrication regime can be helpful for reducing the coefficient of friction. However, based on results (Figures 7-13), it must be considered that increasing texturing parameters to the optimum values in the case of contacts with higher sliding velocities (lubrication regime in hydrodynamic lubrication) may not be as beneficial as at lower velocities. In other words, starvation can limit the beneficial influence of surface texturing at higher velocities. Therefore, surface texturing in the case of starved lubrication may not be advantageous in reducing the friction based on the operating conditions and any limit in lubricant input film.

The parametric study in this article shows that, although the growth in the depth of cavities to the optimum values leads to the higher film thicknesses in the case of starved lubrication, it is possible to limit this growth in lubricant film thickness by limiting the oil supply, as is shown in Figure 9. The same frictional behaviour and merging in Stribeck curves is observable in the case of the study of the effect of size and pitch parameters. Changing the size and pitch parameters has an influence on the coefficient of friction, but for the values close to the optimum value for this parameter, this effect is limited. Furthermore, due to the limit in lubricant supply, the same trend of behaviour can also be made predictable for other patterns, i.e. triangular pockets and circular pockets when the starved lubrication regime is occurring in contact. Moreover, in the case of a chevron pattern similar to the groove pattern, the lowest coefficient of friction in the case of non-starved lubrication is achievable when the depth is around $10 \mu \mathrm{m}$ and the pitch is around 0.5 .

Based on the results for the section on the effect of the input film thickness on the coefficient of friction, this parameter can have a vital role on the efficiency of texturing. In particular, when the input film thickness $\left(h_{\text {oil }}\right)$ is lower than $2.5 \mu \mathrm{m}$, the Stribeck curve transforms into a straight line and the film thickness does not change in comparison with the boundary lubrication regime film thickness.

If the $h_{\text {oil }}$ varies between 2.5 and $8 \mu \mathrm{m}$, the friction level in the $\mathrm{HL}$ and $\mathrm{ML}$ regimes starts to decrease, and the film thickness increases.

The influence of roughness is greater in the boundary lubrication regime and mixed lubrication to the point at which the transition between $\mathrm{ML}$ and $\mathrm{HL}$ occurs. Therefore, employing surfaces with a higher roughness can shift the Stribeck curve; this shift in addition to the effect of starvation can reduce the 
influence of texturing and application of optimized texture properties.

\section{Conclusion}

The goal of this investigation was to study and predict the effect of surface texturing on frictional behaviour for parallel sliding contacts under starved lubrication conditions. In addition, the efficiency of surface texturing as a method to reduce the friction in starved lubricated contact is also studied. This model is a numerical algorithm based on the Reynolds equation with the Elrod cavitation algorithm formulation. By applying the value of calculated film thickness in the deterministic asperity model, the coefficient of friction is calculated. In this article, Stribeck curves for different situations are plotted. The effect of several parameters on starved regime frictional behaviour, such as depth, size and texture pitch, has been studied.

In this study, the deterministic asperity contact model is employed efficiently, considering the effect of different scales of surface features (roughness and texture) on the coefficient of friction that is not dependent on the directions of the asperities.

1. This approach allows the effect of texture and roughness to influence the friction independently; this may be beneficial in optimizing the surface texture.

2. In order to reduce the friction in starved lubrication conditions, surface texturing has a beneficial effect, and this effect is also presented in the numerical study of Gu et al. [33,65].

3. The positive effect is more sensible mostly when contacts are in lower sliding velocities. When the sliding velocity reaches higher values, the effect of texturing can be influenced by the input film thickness.

4. In the case of starved lubrication, when the value of this input film thickness $\left(h_{\text {oil }}\right)$ decreases, the starvation effect gains a greater influence upon the film thickness. Therefore, the effect of variation of the texture parameters (pitch, depth and size) on the coefficient of friction is also decreasing.

5. Surface texturing in starved lubricated conditions, based on operating conditions and a limit in lubricant input film, may not be advantageous as a method to reduce the fiction.

6. In order to apply the texturing in starved lubricated contacts a simulation of the coefficient of friction and film thickness based on texturing and lubricant properties can help to avoid the unnecessary surface texturing.

It is worth mentioning that based on the operating conditions, thermal and atmosphere effects could play an important role in lubrication. These effects should be included in the numerical simulation for accurate performance predictions. However, since these effects were not considered texture-specific, which is the focus of this article, they have not been included in this article.

Author Contributions: Conceptualization, D.J.S., E.L.D. and D.B.; Methodology, D.J.S. and D.B.; Software, D.B.and A.A.; Validation, D.B. and D.J.S.; Formal Analysis, D.B. and D.J.S.; Investigation, D.B. and D.J.S.; Writing-Original Draft Preparation, D.B.; Writing-Review \& Editing, D.B., E.L.D., M.B.d.R. and D.J.S.; Supervision, D.J.S.

Funding: This research was funded by Materials Innovation Institute (M2i) grant number M21.1.11448.

Conflicts of Interest: The authors declare no conflict of interest.

\section{Nomenclature}

\begin{tabular}{|c|c|c|}
\hline Parameters & Description & Unit \\
\hline$A_{C}$ & $\begin{array}{l}\text { Real area of asperity } \\
\text { contact }\end{array}$ & $\mathrm{m}^{2}$ \\
\hline $\mathrm{D}$ & Separation & $\mathrm{m}$ \\
\hline $\mathrm{f}$ & Coefficient of friction & - \\
\hline$f_{C}$ & $\begin{array}{l}\text { Coefficient of friction in } \\
\text { BL regime }\end{array}$ & - \\
\hline$F$ & $\begin{array}{l}\text { Elrod cavitation } \\
\text { algorithm switch } \\
\text { function }\end{array}$ & - \\
\hline$F_{C}$ & $\begin{array}{l}\text { Load carried by the } \\
\text { asperities }\end{array}$ & $\mathrm{N}$ \\
\hline$F_{H}$ & $\begin{array}{l}\text { Load carried by the } \\
\text { hydrodynamic } \\
\text { component }\end{array}$ & $\mathrm{N}$ \\
\hline$F_{f}$ & Friction force & $\mathrm{N}$ \\
\hline$F_{f H}$ & $\begin{array}{l}\text { Hydrodynamic friction } \\
\text { force }\end{array}$ & $\mathrm{N}$ \\
\hline$F_{T}$ & $\begin{array}{l}\text { Normal load on the } \\
\text { contact }\end{array}$ & $\mathrm{N}$ \\
\hline$h$ & Film thickness & $\mathrm{m}$ \\
\hline$h_{\text {oil }}$ & $\begin{array}{l}\text { Limited value of input } \\
\text { film thickness }\end{array}$ & $\mathrm{m}$ \\
\hline$h_{0}$ & Film thickness & $\mathrm{m}$ \\
\hline$H$ & $\begin{array}{l}\text { Dimensionless local } \\
\text { depth of textured } \\
\text { surface }\end{array}$ & - \\
\hline$L_{g x}$ & $\begin{array}{l}\text { Texture cell length in } x \text { - } \\
\text { direction }\end{array}$ & $\mathrm{m}$ \\
\hline$L_{x}$ & $\begin{array}{l}\text { Textured area in } x- \\
\text { direction }\end{array}$ & $\mathrm{m}$ \\
\hline$L_{y}$ & $\begin{array}{l}\text { Textured area in y- } \\
\text { direction }\end{array}$ & $\mathrm{m}$ \\
\hline$p$ & Pressure & $\mathrm{Pa}$ \\
\hline
\end{tabular}




\begin{tabular}{|c|c|c|}
\hline$p_{a}$ & Ambient pressure & $\mathrm{Pa}$ \\
\hline$p_{c}$ & Cavitation pressure & $\mathrm{Pa}$ \\
\hline$P$ & Dimensionless pressure & - \\
\hline$P_{a}$ & $\begin{array}{l}\text { Average contact } \\
\text { pressure }\end{array}$ & $\mathrm{Pa}$ \\
\hline$P_{x}$ & Texture pitch & - \\
\hline$r_{p}$ & $\begin{array}{l}\text { Cavity characteristic } \\
\text { depth }\end{array}$ & $\mathrm{m}$ \\
\hline$S$ & Cavity size $=2 r_{p}$ & $\mathrm{~m}$ \\
\hline$T_{d}$ & Texture depth & $\mathrm{m}$ \\
\hline$U_{0}$ & Sum velocity & - \\
\hline$X$ & $\begin{array}{l}\text { Dimensionless Cartesian } \\
\text { coordination }\end{array}$ & - \\
\hline$Y$ & $\begin{array}{l}\text { Dimensionless Cartesian } \\
\text { coordination }\end{array}$ & - \\
\hline$w_{i}$ & $\begin{array}{l}\text { Compliance of an } \\
\text { asperity }\end{array}$ & $\mathrm{m}$ \\
\hline$z_{i}$ & Asperity height & $\mathrm{m}$ \\
\hline
\end{tabular}

\begin{tabular}{|c|c|c|}
\hline$\rho$ & Density & $\mathrm{kg} \cdot \mathrm{m}^{-3}$ \\
\hline$\gamma_{1}$ & $\begin{array}{l}\text { Adaption parameter for } \\
\text { hydrodynamic } \\
\text { component in } \mathrm{ML}\end{array}$ & - \\
\hline$\gamma_{2}$ & $\begin{array}{l}\text { Adaption parameter for } \\
\text { asperity contact } \\
\text { component in } \mathrm{ML}\end{array}$ & - \\
\hline$\eta$ & Dynamic viscosity & $\mathrm{Pa} \cdot \mathrm{s}$ \\
\hline$\tau_{C}$ & $\begin{array}{l}\text { Shear stress of asperity } \\
\text { contact }\end{array}$ & $\mathrm{Pa}$ \\
\hline$\varphi$ & $\begin{array}{l}\text { Cavitation } \\
\text { dimensionless variable }\end{array}$ & - \\
\hline
\end{tabular}

\section{Appendix A}

Mixed lubrication Model Algorithm

For starved lubrication conditions, the coefficient of friction in the ML regime can be calculated as explained in the flowchart below (see Figure A1). 


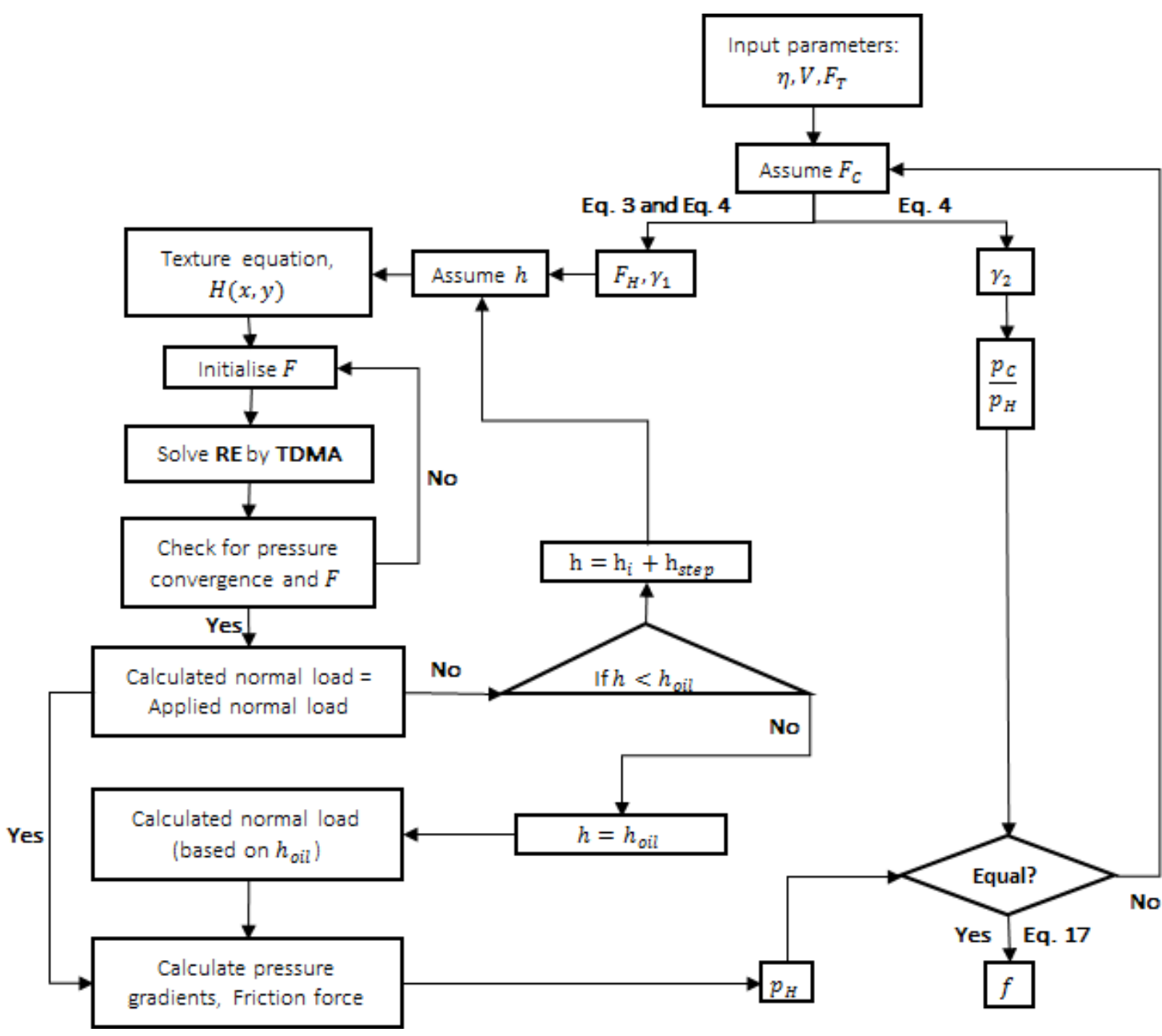

Figure A1. Flow chart of mixed lubrication under starved lubrication conditions.

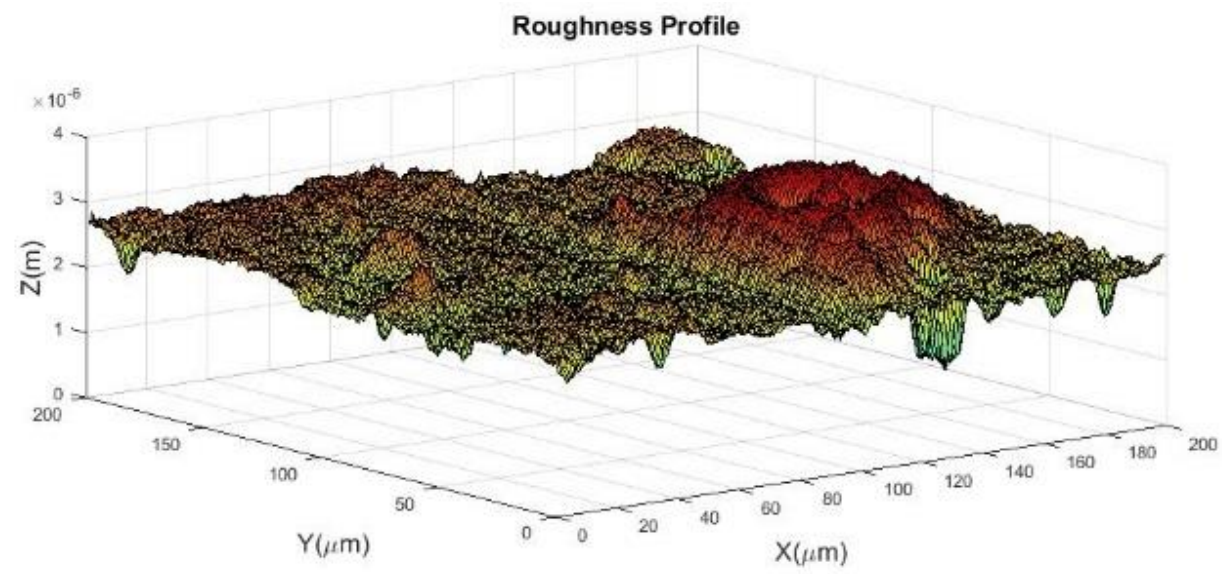

Figure B2. Surface roughness profile based on the Laser Scanning Microscope measurements $\left(R_{a}=0.11 \mu \mathrm{m}\right)$. 


\section{Appendix B}

\section{Determination of Roughness Parameters}

In this study, calculations were performed using the roughness measured by images extracted from the laser microscope; these images are obtained from roughness height measurements. In this appendix, one of these images is shown as an example, and the equations for calculating the roughness parameters are presented.

\section{B.1. Roughness Measurement}

In order to calculate the boundary lubrication component, the roughness measurement is essential. As mentioned in the article, in the case of the deterministic approach the real measured height of asperities is needed so as to calculate the separation between the opposing surfaces. In order to achieve the height of the asperities, the surface topography for textured surfaces is measured by using microscopic images. These analyses have been performed using a Keyence Color 3D Scanning Microscope (Keyence, Osaka, Japan), which uses a violet Laser $\lambda=388 \mathrm{~nm}$. The result from a roughness measurement in the case of a chevron textured sample is presented in Figure B1, (standard lens $50 \times$ is employed).

200. 0

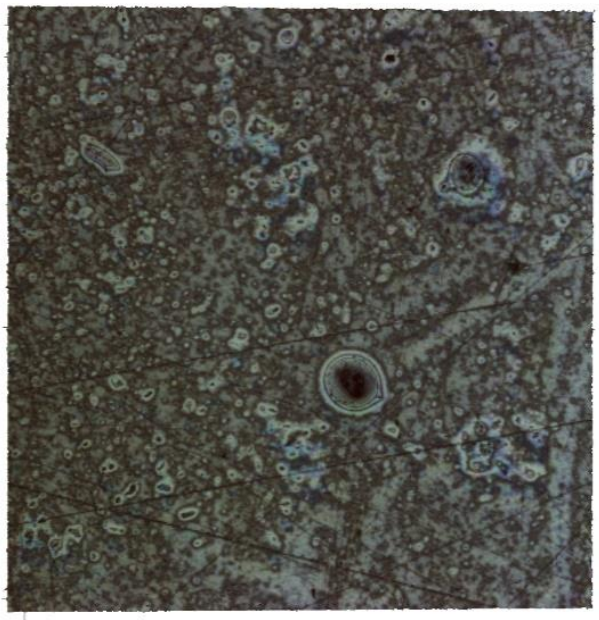

0. $0 \mu \mathrm{m}$

$$
\text { 0. } 0 \mu \mathrm{m}
$$

100. 0

200. 0

Figure B1. Surface image by Laser Scanning Microscope.

The roughness data measured by confocal microscope is illustrated in Figure B2.

Figure B3 shows the real measured probability density of asperities against the dimensionless asperity height, in order to compare the measured roughness with the Gaussian roughness distribution. The red line represents the Gaussian probability density distribution of one surface.

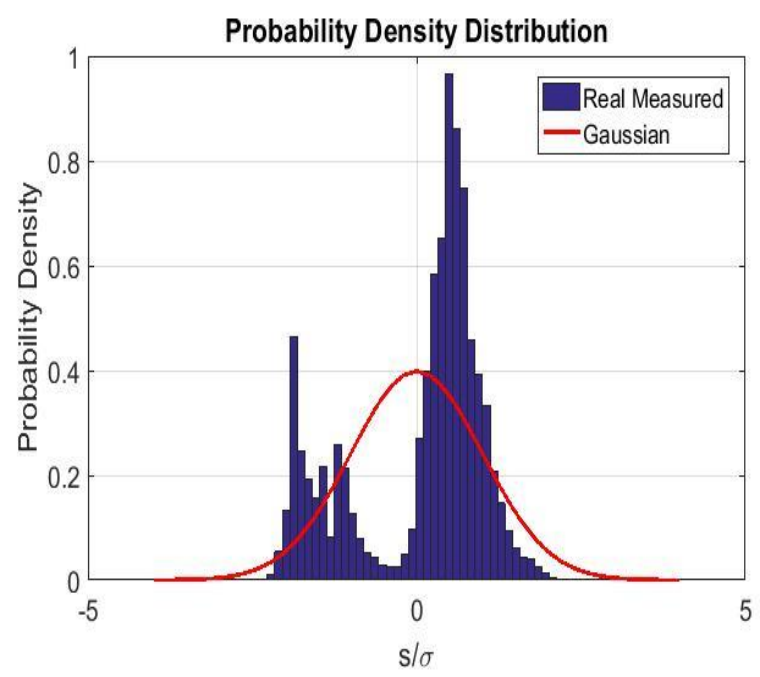

Figure B3. Real measured and Gaussian distribution of surface heights as a function of the dimensionless asperity height $(s / \sigma)$.

The Summit is defined as a point that is higher than its eight neighbour points (see Figure B4).

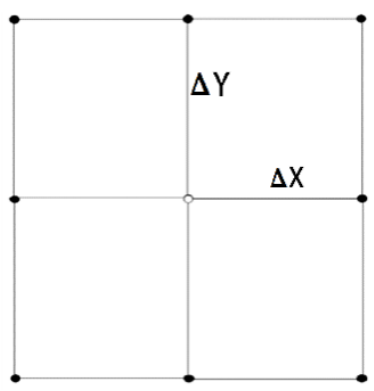

Figure B4. Definition of a Summit.

To determine the radius of an asperity the threepoint definition is employed. In Figure B4, $\Delta x, \Delta y$ are the steps or pixel size, and the asperity radii in both the $x$ and $y$ directions can be calculated as:

$$
\begin{aligned}
& \beta_{x}^{-1}=\frac{z_{x-\Delta x, y}-2 z_{x, y}+z_{x+\Delta x, y}}{\Delta x^{2}} \\
& \beta_{y}^{-1}=\frac{z_{x-\Delta x, y}-2 z_{x, y}+z_{x+\Delta x, y}}{\Delta y^{2}}
\end{aligned}
$$

In Equation $\mathrm{B} 1, \beta_{x}$ is the asperity radii in $\mathrm{x}$ direction; in Equation $\mathrm{B} 2, \beta_{y}$ is the asperity radii in $y$ direction and $z_{x, y}$ is the local surface height at location $(x, y)$. The combined summit radius $\beta_{i}$ of the radii in the two perpendicular directions $\beta_{x}$ and $\beta_{y}$ is obtained by:

$$
\beta_{i}=\sqrt{\beta_{x i} \cdot \beta_{y i}}
$$


To calculate the average summit radius $(\bar{\beta})$ we have:

$$
\bar{\beta}=\frac{1}{n} \sum_{i=1}^{n} \beta_{i}
$$

Therefore, the calculated average radius of asperity $(\bar{\beta})$, is equal to $4.8 \times 10^{-8} \mathrm{~m}$.

\section{References}

[1] Evans, H. P., Snidle, R. W., and Sharif, K. J., 2009, "Deterministic mixed lubrication modelling using roughness measurements in gear applications," Tribology International, 42(10), pp. 1406-1417.

[2] Faraon, I., and Schipper, D. J., 2007, "Stribeck curve for starved line contacts," Journal of Tribology, 129(1), pp. 181-187.

[3] Gethin, D., and Medwell, J., 1987, "Paper III (iv) Starvation effects in two high speed bearing types," Tribology series, 11, pp. 81-92.

[4] Eugen, B. T., and Sandi, B. C., 2012, "Specific problems of high speed bearings," Journal of Engineering Studies and Research, 18(1).

[5] Olaru, D., and Gafitanu, M., 1997, "A new methodology to estimate starvation in ball bearings," Lubrication Science, 4(1), pp. 93-106.

[6] Wedeven, L. D., Evans, D., and Cameron, A., 1971, "Optical analysis of ball bearing starvation," J Lubric Technol Trans ASME, 93 Ser F(3), pp. 349363.

[7] Pemberton, J., and Cameron, A., 1976, "A mechanism of fluid replenishment in elastohydrodynamic contacts," Wear, 37(1), pp. 185-190.

[8] Kingsbury, E., 1973, "Cross flow in a starved EHD contact," ASLE Transactions, 16(4), pp. 276-280.

[9] Chiu, Y. P., 1974, "An analysis and prediction of lubricant film starvation in rolling contact systems," ASLE Transactions, 17(1), pp. 22-35.

[10] Damiens, B., Venner, C. H., Cann, P. M. E., and Lubrecht, A. A., 2004, "Starved lubrication of elliptical EHD contacts," Journal of Tribology, 126(1), pp. 105-111.

[11] Chevalier, F., Lubrecht, A. A., Cann, P. M. E., Colin, F., and Dalmaz, G., 1998, "Film thickness in starved EHL point contacts," Journal of Tribology, 120(1), pp. 126-132.

[12] Jakobsson, B., and Floberg, L., 1957, The finite journal bearing, considering vaporization: (Das Gleitlager von endlicher Breite mit Verdampfung), Chalmers University of Technology, Chalmers Tekniska Högskolas handlingar ; Avd. Maskinteknik

[13] Olsson, K. O., 1965, Cavitation in Dynamically Loaded Bearings, Scandinavian University Books, Chalmers University of Technology, Institute of Machine Elements. Report.
[14] Brewe, D. E., and Hamrock, B. J., 1982, "Analysis of starvation effects on hydrodynamic lubrication in nonconforming contacts," TRANS. ASME J. LUBR. TECHNOL., 104(3 , Jul. 1982), pp. 410-417.

[15] Boness, R. J., 1970, "Effect of oil supply on cage and roller motion in a lubricated roller bearing.," J Lubric Technol Trans ASME, 92 Ser F(1), pp. 39-53.

[16] Chevalier, F., Lubrecht, A. A., Cann, P. M. E., Colin, F., and Dalmaz, G., 1995, "Starved Film Thickness: a Qualitative Explanation," Tribology Series, C. M. T. T. H. C. C. D. Dowson, and G. Dalmaz, eds., Elsevier, pp. 249-257.

[17] Elrod, H. G., 1981, "A Cavitation algorithm," Journal of lubrication technology, 103(3), pp. 350354.

[18] Elrod, H. G., and Adams, M. L., 1975, "A computer program for cavitation and starvation problems.."

[19] Cann, P. M. E., and Lubrecht, A. A., 2007, "Bearing performance limits with grease lubrication: The interaction of bearing design, operating conditions and grease properties," Journal of Physics D: Applied Physics, 40(18), pp. 5446-5451.

[20] Hirst, W., 1974, "Scuffing and its prevention," Chart Mech Eng, 21(4), pp. 88-92.

[21] Glavatskih, S. B., McCarthy, D. M. C., and Sherrington, I., 2005, "Hydrodynamic Performance of a Thrust Bearing with Micropatterned Pads," Tribology Transactions, 48(4), pp. 492-498.

[22] Brizmer, V., Kligerman, Y., and Etsion, I., 2003, "A Laser Surface Textured Parallel Thrust Bearing," Tribology Transactions, 46(3), pp. 397-403.

[23] Etsion, I., 2010, "State of the Art in Laser Surface Texturing," Advanced Tribology, J. Luo, Y. Meng, T. Shao, and Q. Zhao, eds., Springer Berlin Heidelberg, pp. 761-762.

[24] Etsion, I., and Burstein, L., 1996, "A Model for Mechanical Seals with Regular Microsurface Structure," Tribology Transactions, 39(3), pp. 677683.

[25] Etsion, I., Halperin, G., Brizmer, V., and Kligerman, Y., 2004, "Experimental Investigation of Laser Surface Textured Parallel Thrust Bearings," Tribology Letters, 17(2), pp. 295-300.

[26] Etsion, I., Kligerman, Y., and Halperin, G., 1999, "Analytical and Experimental Investigation of Laser-Textured Mechanical Seal Faces," Tribology Transactions, 42(3), pp. 511-516.

[27] Kovalchenko, A., Ajayi, O., Erdemir, A., Fenske, G., and Etsion, I., 2005, "The effect of laser surface texturing on transitions in lubrication regimes during unidirectional sliding contact," Tribology International, 38(3), pp. 219-225.

[28] Ronen, A., Etsion, I., and Kligerman, Y., 2001, "Friction-Reducing Surface-Texturing in 
Reciprocating Automotive Components," Tribology Transactions, 44(3), pp. 359-366.

[29] Ryk, G., Kligerman, Y., and Etsion, I., 2002, "Experimental Investigation of Laser Surface Texturing for Reciprocating Automotive Components," Tribology Transactions, 45(4), pp. 444-449.

[30] Briscoe, B., Scruton, B., and Willis, F., 1973, "The shear strength of thin lubricant films," Proceedings of the Royal Society of London. A. Mathematical and Physical Sciences, 333(1592), pp. 99-114.

[31] Kango, S., Sharma, R., and Pandey, R., 2014, "Thermal analysis of microtextured journal bearing using non-Newtonian rheology of lubricant and JFO boundary conditions," Tribology International, 69, pp. 19-29.

[32] Guzek, A., Podsiadlo, P., and Stachowiak, G. W., 2013, "Optimization of textured surface in 2D parallel bearings governed by the Reynolds equation including cavitation and temperature," Tribology Online, 8(1), pp. 7-21.

[33] Gu, C., Meng, X., Xie, Y., and Kong, X., 2017, "Performance of surface texturing during start-up under starved and mixed lubrication," Journal of Tribology, 139(1), p. 011702.

[34] Bijani, D., Deladi, E., Akchurin, A., de Rooij, M., and Schipper, D., 2018, "The influence of surface texturing on the frictional behaviour of parallel sliding lubricated surfaces under conditions of mixed lubrication," Lubricants, 6(4), p. 91.

[35] Hamilton, D. B., Walowit, J. A., and Allen, C. M., 1966, "A Theory of Lubrication by Microirregularities," Journal of Basic Engineering, 88(1), pp. 177-185.

[36] Qiu, Y., and Khonsari, M. M., 2011, "Experimental investigation of tribological performance of laser textured stainless steel rings," Tribology International, 44(5), pp. 635-644.

[37] Kovalchenko, A., Ajayi, O., Erdemir, A., Fenske, G., and Etsion, I., 2004, "The effect of laser texturing of steel surfaces and speed-load parameters on the transition of lubrication regime from boundary to hydrodynamic," Tribology Transactions, 47(2), pp. 299-307.

[38] Raeymaekers, B., Etsion, I., and Talke, F. E., 2007, "Enhancing tribological performance of the magnetic tape/guide interface by laser surface texturing," Tribology Letters, 27(1), pp. 89-95.

[39] Johnson, K. L., Greenwood, J. A., and Poon, S. Y., 1972, "A simple theory of asperity contact in elastohydro-dynamic lubrication," Wear, 19(1), pp. 91-108.

[40] Greenwood, J. A., and Williamson, J. B. P., 1966, "Contact of Nominally Flat Surfaces," Proceedings of the Royal Society of London. Series A.
Mathematical and Physical Sciences, 295(1442), pp. 300-319.

[41] Gelinck, E. R. M., 1999, "Mixed lubrication of line contacts,"PhD thesis, University of Twente, Enschede.

[42] Shi, F., and Salant, R. F., 2000, "A mixed soft elastohydrodynamic lubrication model with interasperity cavitation and surface shear deformation," Journal of Tribology, 122(1), pp. 308-316.

[43] Patir, N., and Cheng, H. S., 1978, "Application of Average Flow Model to Lubrication Between Rough Sliding Surfaces," Journal of Lubrication Technology, 101, p. 10.

[44] Patir, N., and Cheng, H. S., 1978, "An Average Flow Model for Determining Effects of ThreeDimensional Roughness on Partial Hydrodynamic Lubrication," Journal of Lubrication Technology, 100(1), pp. 12-17.

[45] Hu, Y.-Z., Barber, G. C., and Zhu, D., 1999, "Numerical analysis for the elastic contact of real rough surfaces," Tribology transactions, 42(3), pp. 443-452.

[46] Hu, Y.-Z., and Zhu, D., 2000, "A full numerical solution to the mixed lubrication in point contacts," Journal of Tribology, 122(1), pp. 1-9.

[47] Hu, Y.-z., Wang, H., Wang, W.-z., and Zhu, D., 2001, "A computer model of mixed lubrication in point contacts," Tribology International, 34(1), pp. 65-73.

[48] Faraon, I. C., 2005, "Mixed lubricated line contacts, PhD thesis,"PhD Thesis, University of Twente, Enschede.

[49] Wakuda, M., Yamauchi, Y., Kanzaki, S., and Yasuda, Y., 2003, "Effect of surface texturing on friction reduction between ceramic and steel materials under lubricated sliding contact," Wear, 254(3-4), pp. 356-363.

[50] Wang, W.-Z., Huang, Z., Shen, D., Kong, L., and Li, S., 2013, "The effect of triangle-shaped surface textures on the performance of the lubricated point-contacts," Journal of Tribology, 135.

[51] Wang, X., Kato, K., Adachi, K., and Aizawa, K., 2001, "The effect of laser texturing of SiC surface on the critical load for the transition of water lubrication mode from hydrodynamic to mixed," Tribology International, 34(10), pp. 703-711.

[52] Etsion, I., 2004, "Improving tribological performance of mechanical components by laser surface texturing," Tribology Letters, 17(4), pp. 733-737.

[53] Ryk, G., and Etsion, I., 2006, "Testing piston rings with partial laser surface texturing for friction reduction," Wear, 261(7-8), pp. 792-796.

[54] Vilhena, L. M., Sedlaček, M., Podgornik, B., Vižintin, J., Babnik, A., and Možina, J., 2009, 
"Surface texturing by pulsed Nd:YAG laser," Tribology International, 42(10), pp. 1496-1504.

[55] Dunn, A., Wlodarczyk, K., Carstensen, J., Hansen, E., Gabzdyl, J., Harrison, P., Shephard, J., and Hand, D., 2015, "Laser surface texturing for high friction contacts," Applied Surface Science, 357, pp. 2313-2319.

[56] Saeidi, F., Parlinska-Wojtan, M., Hoffmann, P., and Wasmer, K., 2017, "Effects of laser surface texturing on the wear and failure mechanism of grey cast iron reciprocating against steel under starved lubrication conditions," Wear, 386-387, pp. 29-38.

[57] Bijani, D., Deladi, L. E., and Schipper, D. J., 2016, "The Influence of Surface Texturing on the Film Thickness in Parallel Sliding Surfaces," 20th International Colloquium Tribology: Technische Akademie Esslingen, p. 12.

[58] Coyne, J. C., and Elrod, H. G., 1965, Conditions for the Rupture of a Lubrication Film.

[59] Xiong, S., and Wang, Q. J., 2012, "Steady-state hydrodynamic lubrication modeled with the payvar-salant mass conservation model," Journal of Tribology, 134(3).

[60] Qiu, M., Minson, B. R., and Raeymaekers, B., 2013, "The effect of texture shape on the friction coefficient and stiffness of gas-lubricated parallel slider bearings," Tribology International, 67, pp. 278-288.

[61] Qiu, M., Delic, A., and Raeymaekers, B., 2012, "The effect of texture shape on the load-carrying capacity of gas-lubricated parallel slider bearings," Tribology Letters, 48(3), pp. 315-327.

[62] Patankar, S. V., 1980, Numerical heat transfer and fluid flow / Suhas V. Patankar, Hemisphere Pub. Corp. ; McGraw-Hill, Washington : New York.

[63] Versteeg, H. K., and Malalasekera, W., 1995, An Introduction to Computational Fluid Dynamics. The Finite Volume Method, Longman Group Ltd.

[64] Bijani, D., Deladi, E., de Rooij, M., and Schipper, D., 2018, "The influence of surface texturing on the film thickness in starved lubricated parallel sliding contacts," Lubricants, 6(3), p. 61

[65] Gu, C., Meng, X., Xie, Y., and Yang, Y., 2016, "Effects of surface texturing on ring/liner friction under starved lubrication," Tribology international, 94, pp. 591-605. 


\title{
Paper E
}

\section{The influence of surface texturing and boundary slip on the film thickness in parallel sliding surfaces}

\author{
Dariush Bijani, M.B. de Rooij , E.L. Deladi and D.J. Schipper
}

TAE 2018 Proceedings

The Influence of Surface Texturing and Boundary Slip on the Film Thickness in Parallel Sliding Surfaces

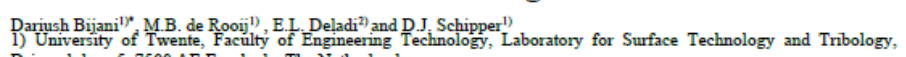
Drienerlolaan $5,7500 \mathrm{AE}$ Enschede, The Netherlands
2) Bosch Transmission technology, Dr. Hub Van Doorneweg 120,5026 RA, Tilburg, The Netherlands

*Corresponding author. Email: d.bijani@utwentenl

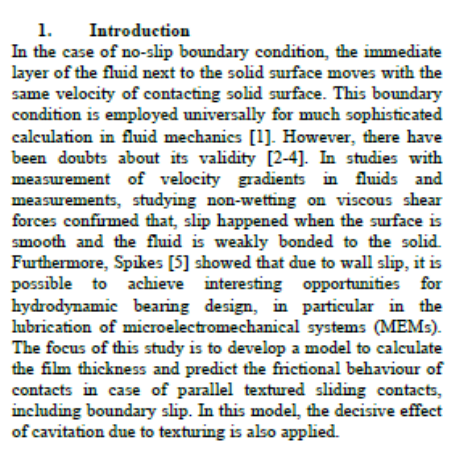

2. Mathematical solution

Experimental studies with wall slip make use of surfaces with different physical or chemical treatments together different solid/liquid interfacial properties Another common means to change the property of the solid/liquid interface is by addition of surfactants to the liquid lubricant. The velocity of the fluid flow at the wall surface can be effectively adjusted by putting different concentrations of surfactants $[6]$

A boundary condition for partial slip was proposed by Navier in 1823. This boundary condition is the most frequently used model to charactenze boundary slip. Naver ing a lis

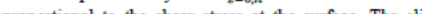
length, $b$, which is defined as the distance beyond the liquid/solid interface as shown in Fig. 1.b.

Where, $b$ is slip length, $u_{s}$ is slip velocity of the fluid $u_{z=0, h}=u_{s}=b \frac{\tau_{x}}{\eta}=\left.b \frac{d u}{d z}\right|_{z=0, h}$

along the surface $\mathrm{x}$-axis, $u$ is velocity component in $\mathrm{x}$ direction,

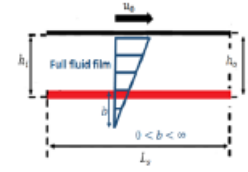

Figure 1: Schematic of wall slip model.

Spikes and Granick [1] introduced a new mathematical slip model for Newtonian fluids. Their model $\tau_{c}=\tau_{c o}+\frac{\eta}{b} u_{s}$ incorporates both the critical shear stress and constant slip length critena in which $\tau_{c o}$ is the critical shear stress at the onset of slip. The critical shear stress model adopted in the present study assumes that wall slip occurs only after the surface shear stress reaches the critical shear stress. The focus of this section is to show the importance of the critical shear stress choice for contacts

$X=\frac{x}{L}, Y=\frac{y}{B}, H=\frac{h}{h_{0}}, U_{s}=\frac{u_{s}}{u_{0}}, V_{s}=\frac{v_{s}}{u_{s}}, P$ $=\frac{p-p_{c}}{p_{\mathrm{a}}}, W=\frac{6 \eta u_{0} L^{2}}{p_{\mathrm{a}} h_{0}^{2}}$

By applying the boundary equations it is possible to reach to Eq 2 .

$\frac{\partial}{\partial X}\left(H^{3} \frac{\partial P}{\partial X}\right)+\frac{L^{2}}{B^{2}} \frac{\partial}{\partial Y}\left(H^{3} \frac{\partial P}{\partial Y}\right)=$

$W\left(\frac{1}{L} \frac{\partial H}{\partial X}+\frac{1}{L} \frac{\partial\left(H U_{s}\right)}{\partial X}+\frac{1}{B} \frac{\partial\left(H V_{s}\right)}{\partial Y}\right)$

The optimum slip area dimensions from work of Tauviginahman et al. [7] is applied to achieve a higher film thickness. For the boundary conditions for slip area we

$\left\{\begin{array}{l}z=h \rightarrow u=u_{s}, v=v_{s} \\ z=0 \rightarrow u=u_{0}, v=0\end{array}\right.$

(3)

$h_{h_{i}}=h_{o}=h$

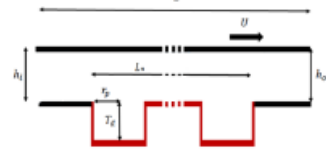

Figure 2: The schematic parallel sliding contacts, the red lines are showing the surfaces with boundary slip effect. 



\title{
The influence of surface texturing and boundary slip on the film thickness in parallel sliding surfaces
}

\author{
Dariush Bijani $^{1)^{*}}$, M.B. de Rooij ${ }^{1)}$, E.L. Deladi ${ }^{2)}$ and D.J. Schipper ${ }^{1)}$ \\ 1) University of Twente, Faculty of Engineering Technology, Laboratory for Surface Technology and \\ Tribology, Drienerlolaan 5, 7500 AE Enschede, The Netherlands
}

2) Bosch Transmission technology, Dr. Hub Van Doorneweg 120, 5026 RA, Tilburg, The Netherlands

*Corresponding author. Email: d.bijani@utwente.nl

\begin{abstract}
The influence of wall slip on fluid flow is of interest for many engineering topics involving liquid-solid interfacial phenomena, such as flow through porous media, liquid coatings and lubrication. Boundary slip can act as a method to reduce friction in lubricated contacts. Furthermore, surface texturing can also play a positive role in enhancing the film thickness. Therefore, a combination of these two methods can be beneficial in order to improve the tribological performance of lubricated contacts. Although the study of frictional behaviour of surfaces with boundary slip has been getting more attention in recent years, the combination of these two methods of surface property modification is interesting to investigate. For the no-slip boundary condition, the first layer of fluid molecules has the same velocity as the contacting solid surface and this condition has been widely applied in the field of fluid mechanics. In lubricated contacts with boundary slip, the first layer of the lubricant molecules moves at a different velocity from the solid surface. In this article, the frictional behaviour of boundary slip in parallel sliding lubricated contacts for textured surfaces is investigated. This study shows that boundary slip has a significant influence on the film thickness in the case of textured surfaces. Furthermore, it is possible to increase the film thickness, and enhance the frictional behaviour by modifying the boundary slip parameters.
\end{abstract}

\section{Keywords}

Hydrodynamic lubrication; surface texturing; boundary slip; film thickness; texturing patterns; numerical modelling

\section{Nomenclature}

\begin{tabular}{llc}
\hline Parameters & Description & Unit \\
\hline$\rho$ & Lubricant density in full film region & $\mathrm{Kg} \cdot \mathrm{m}^{-3}$ \\
$\eta$ & Dynamic viscosity & $\mathrm{Pa} \cdot \mathrm{s}$ \\
$\varphi$ & Cavitation dimensionless variable & - \\
$\mu$ & Coefficient of friction & - \\
$\mu_{r e f}$ & Coefficient of friction for reference condition & - \\
$\mu^{*}$ & Normalized coefficient of friction for slip length study $=\mu / \mu_{r e f}$ & - \\
$\tau_{x z}$ & Shear stress & $P a$ \\
$\tau_{c x}$ & Shear stress x-component when slip takes place at solid surface & $P a$ \\
$\tau_{c o}$ & Critical shear stress & $P a$ \\
$b$ & Slip length & $m$ \\
$F$ & Elrod cavitation algorithm switch function & - \\
$H$ & Dimensionless local depth of textured surface & - \\
\hline
\end{tabular}




\begin{tabular}{|c|c|c|}
\hline$h$ & Film thickness & $m$ \\
\hline$h_{0}$ & Contact separation & $m$ \\
\hline$L$ & Contact length & $m$ \\
\hline$L_{s}$ & Slip area length & $m$ \\
\hline$L_{g x}$ & Texture cell length in $\mathrm{x}$-direction & $m$ \\
\hline$L_{g y}$ & Texture cell length in y-direction & $m$ \\
\hline$L_{x}$ & Textured length in $\mathrm{x}$-direction & $m$ \\
\hline$L_{y}$ & Textured length in y-direction & $m$ \\
\hline$P$ & Dimensionless pressure & - \\
\hline$p$ & Pressure & $P a$ \\
\hline$p_{a}$ & Ambient pressure & $P a$ \\
\hline$p_{c}$ & Cavitation pressure & $P a$ \\
\hline$P_{x}$ & Texture pitch & - \\
\hline$r_{p}$ & Cavity characteristic width & $m$ \\
\hline$S$ & Cavity size $=2 r_{p}$ & $m$ \\
\hline$T_{d}$ & Texture depth & $m$ \\
\hline$u_{0}$ & Velocity & $m \cdot s^{-1}$ \\
\hline$u_{s}$ & Slip velocity in $\mathrm{x}$-direction & $m \cdot s^{-1}$ \\
\hline$v_{S}$ & Slip velocity in y-direction & $m \cdot s^{-1}$ \\
\hline$X$ & Dimensionless Cartesian coordination $=\mathrm{x} / \mathrm{r}_{\mathrm{p}}$ & - \\
\hline$Y$ & Dimensionless Cartesian coordination $=y / r_{p}$ & - \\
\hline
\end{tabular}

\section{Introduction}

In the case of a no-slip boundary condition, the immediate layer of the fluid next to the solid surface moves at the same velocity as the contacting solid surface. This boundary condition is employed universally in many sophisticated calculations in fluid mechanics [1]. However, there have been doubts about its validity [2-6]. Studies involving the measurement of velocity gradients in fluids $[7,8]$ and those involving the measurement of non-wetting surfaces and viscous shear forces $[9,10]$ confirmed that slip occurred when the surface is smooth and the fluid is weakly bonded to the solid [7, 8, 11-13]. Furthermore, Spikes [14, 15] showed that, due to wall slip, it is possible to achieve interesting opportunities for hydrodynamic bearing design, in particular in the lubrication of microelectromechanical systems (MEMs).

The effect of boundary slip on friction reduction is investigated In the study of Tauviqirrahman et al. [16]. In that study a combined optimized complex slip surface and an optimized slope incline ratio are obtained. It was shown that surface optimization of a parallel sliding gap with a slip surface can double the hydrodynamic load-carrying capacity and reduce the friction force by half of what the classical Reynolds theory predicts for an optimal slope inclination of a traditional sider contact. For a two-dimensional (finite length) journal bearing, Ma et al. [17] showed that the optimization of shape and size of the surface may enhance the frictional behaviour.

It has been found that some non-Newtonian liquids such as polymer melts show boundary slip effects at solid surfaces. This boundary slip is due to reduction in viscosity of the fluid layer close to the surface as a result of shear thinning or compositional variation [18-21]. In these cases, slip has been modelled by the Navier slip length model. The results in these studies show an agreement with the predictions and measurements [1]. For Newtonian liquids, the results were compared with this Navier model. Some studies obtained results were closely in line with the constant slip length model $[7,8$, 13] but others were not [12, 22-24].

To solve this issue, a wall slip model was developed by Spikes [15] in which a critical shear stress criterion is broadened to incorporate both a critical shear stress and a constant slip length criterion. Comparing this combined model with experiments showed rather good agreement.

Tauviqirrahman et al. $[25,26]$ showed that when the surface has partial boundary slip, it is possible to improve the performance of fluid bearings by 
increasing the lubricant film thickness due to the higher load-carrying capacity generation. In these studies, the lubricated sliding contact is operating under steady state conditions.

The focus of this study is to develop a model to calculate the film thickness and predict the frictional behaviour of contacts in case of parallel textured sliding contacts, including boundary slip. In this model, the decisive effect of cavitation due to texturing is also applied.

\section{Mathematical solution}

Experimental studies with wall slip make use of surfaces with different physical or chemical treatments together with different types of lubricants in order to achieve different solid/liquid interfacial properties. Another common method of changing the property of the solid/liquid interface is by adding surfactants to the liquid lubricant. The velocity of the fluid flow at the wall surface can be adjusted effectively by using different concentrations of surfactants [24].

In order to simulate the boundary slip phenomenon the main challenge is choosing the most realistic slip model and employing the most accurate boundary conditions for a hydrophobic surface.

Two main wall slip models have been adopted to describe the boundary slip: the slip length model (SLM) and the critical shear stress model (CSSM)[16].

\section{Slip length model (SLM):}

A boundary condition for partial slip was proposed by Navier in 1823. This boundary condition is the most frequently used model to characterize boundary slip. Navier introduced a linear boundary condition, which states that the liquid velocity term $\mathrm{u}_{\mathrm{z}=0, \mathrm{~h}}$ at the wall surfaces is proportional to the shear stress at the surface.

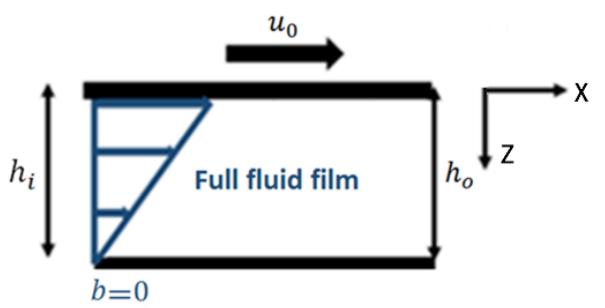

(a)

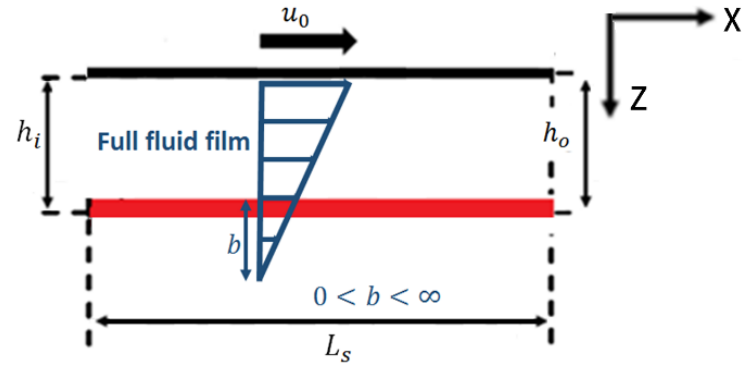

(b)

Figure 1. Schematic of wall slip model. (a) No-slip, (b) Boundary slip condition.

The slip length, $b$, is defined as the distance beyond the liquid/solid interface, as shown in Fig. 1.b.

$$
\mathrm{u}_{\mathrm{z}=\mathrm{h}}=\mathrm{u}_{\mathrm{s}}=\mathrm{b} \frac{\tau_{\mathrm{x}}}{\eta}=\left.\mathrm{b} \frac{\mathrm{du}}{\mathrm{dz}}\right|_{\mathrm{z}=\mathrm{h}}
$$

Where:

$u_{s}$ : Slip velocity of the fluid along the surface $x$-axis

$\mathrm{u}$ : Velocity component in $\mathrm{x}$-direction

$\eta$ : Bulk viscosity

$\tau_{\mathrm{x}}$ : Shear stress in $\mathrm{x}$-direction.

Critical shear stress model (CSSM):

Spikes and Granick [1] introduced a new mathematical slip model for Newtonian fluids. Their model $\tau_{c}=$ $\tau_{c o}+\frac{\eta}{b} u_{s}$ incorporates both the critical shear stress and the constant slip length criteria in which $\tau_{\text {co }}$ is the critical shear stress at the onset of slip. The critical shear stress model adopted in the present study assumes that wall slip occurs only after the surface shear stress reaches the critical shear stress. The focus of this section is on showing the importance of the critical shear stress choice for contacts with a textured surface combined with a slip boundary.

\subsection{Governing equations}

Based on the force equilibrium analysis of a fluid element one obtains:

$$
\frac{\partial p}{\partial x}=\frac{\partial \tau}{\partial z}
$$

For Newtonian flow:

$$
\tau=\eta \frac{\partial u}{\partial z}
$$

Substituting Eq. 2 in Eq. 3 and by applying the flow continuity the result is: 


$$
\begin{aligned}
\rightarrow \frac{\partial \mathrm{q}_{\mathrm{x}}}{\partial \mathrm{x}}+\frac{\partial \mathrm{q}_{\mathrm{y}}}{\partial \mathrm{y}}=0 & \\
\rightarrow & \frac{\partial}{\partial \mathrm{x}}\left(\frac{-\mathrm{h}^{3}}{12 \eta} \frac{\partial \mathrm{p}}{\partial \mathrm{x}}\right)+\frac{\partial}{\partial \mathrm{x}}\left(\frac{\mathrm{h}}{2}\left(\mathrm{u}_{0}+\mathrm{u}_{\mathrm{s}}\right)\right) \\
+ & \frac{\partial}{\partial \mathrm{y}}\left(\frac{-\mathrm{h}^{3}}{12 \eta} \frac{\partial \mathrm{p}}{\partial \mathrm{y}} \mathrm{h}^{3}\right)+\frac{\partial}{\partial \mathrm{y}}\left(\frac{\mathrm{v}_{\mathrm{s}} \mathrm{h}}{2}\right)=0 \\
\frac{\partial}{\partial \mathrm{x}}\left(\mathrm{h}^{3} \frac{\partial \mathrm{p}}{\partial \mathrm{x}}\right)+\frac{\partial}{\partial \mathrm{y}}\left(\mathrm{h}^{3} \frac{\partial \mathrm{p}}{\partial \mathrm{y}}\right) & 6 \mathrm{~h}\left(\mathrm{u}_{0} \frac{\partial \mathrm{h}}{\partial \mathrm{x}}+\frac{\partial\left(\mathrm{hu}_{\mathrm{s}}\right)}{\partial \mathrm{x}}\right. \\
& \left.+\frac{\partial\left(\mathrm{v}_{\mathrm{s}} \mathrm{h}\right)}{\partial \mathrm{y}}\right)
\end{aligned}
$$

By defining the following dimensionless parameters:

$$
\begin{aligned}
X=\frac{\mathrm{X}}{\mathrm{L}}, \mathrm{Y}=\frac{\mathrm{y}}{\mathrm{B}}, \mathrm{H} & =\frac{\mathrm{h}}{\mathrm{h}_{0}}, \mathrm{U}_{\mathrm{s}}=\frac{\mathrm{u}_{\mathrm{s}}}{\mathrm{u}_{0}}, \mathrm{~V}_{\mathrm{s}}=\frac{\mathrm{v}_{\mathrm{s}}}{\mathrm{u}_{0}}, \mathrm{P} \\
& =\frac{\mathrm{p}-\mathrm{p}_{\mathrm{c}}}{\mathrm{p}_{\mathrm{a}}}, \mathrm{W}=\frac{6 \eta \mathrm{u}_{0} \mathrm{~L}^{2}}{\mathrm{p}_{\mathrm{a}} \mathrm{h}_{0}^{2}}
\end{aligned}
$$

Eq. 4 becomes:

$$
\begin{aligned}
\frac{\partial}{\partial \mathrm{X}}\left(\mathrm{H}^{3} \frac{\partial \mathrm{P}}{\partial \mathrm{X}}\right) & +\frac{\mathrm{L}^{2}}{\mathrm{~B}^{2}} \frac{\partial}{\partial \mathrm{Y}}\left(\mathrm{H}^{3} \frac{\partial \mathrm{P}}{\partial \mathrm{Y}}\right) \\
& =\mathrm{W}\left(\frac{1}{\mathrm{~L}} \frac{\partial \mathrm{H}}{\partial \mathrm{X}}+\frac{1}{\mathrm{~L}} \frac{\partial\left(\mathrm{HU}_{\mathrm{s}}\right)}{\partial \mathrm{X}}\right. \\
& \left.+\frac{1}{\mathrm{~B}} \frac{\partial\left(\mathrm{HV}_{\mathrm{s}}\right)}{\partial \mathrm{Y}}\right)
\end{aligned}
$$

\subsection{Boundary conditions2.3.1 Slip Length Model (SLM)}

By applying boundary slip on the stationary surface it is possible to increase the tribological behaviour [16, 2527 i.e. to achieve a higher load-carrying capacity for the parallel sliding surface situation (see Fig. 2).

In addition, the slip area starts from the point where the flow enters the first cavity; the reason for using this geometry is to apply the effect of partial slip. Furthermore, the optimum slip area dimensions from work of Tauviqirrahman et al. [28] is applied to achieve a higher film thickness. Tauviqirrahman et al. [25] showed in their study how to achieve a thicker lubricant with an optimized partial slip surface area. Therefore, for the boundary conditions for slip area in Eq. 6 we have:

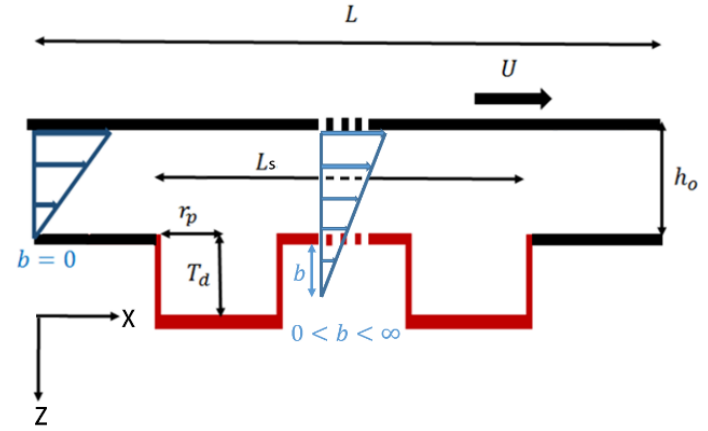

Figure 2. Parallel sliding contact (schematic), the red lines indicates part of the surface with boundary slip.

$$
\left\{\begin{array}{l}
\mathrm{z}=\mathrm{h} \rightarrow \mathrm{u}=\mathrm{u}_{\mathrm{s}}, \mathrm{v}=\mathrm{v}_{\mathrm{s}} \\
\mathrm{z}=0 \rightarrow \mathrm{u}=\mathrm{u}_{0}, \mathrm{v}=0 \\
\mathrm{~h}_{\mathrm{i}}=\mathrm{h}_{\mathrm{o}}=\mathrm{h}
\end{array}\right.
$$

2.3.2 Critical Shear Stress Model (CSSM) The two-component slip model reads:

$$
\tau_{c}=\tau_{c o}+\frac{\eta}{b} u_{s}
$$

From the boundary conditions in the case where slip occurs at the lower stationary surface, the upper and lower surfaces boundary condition reads:

$$
\left\{\begin{array}{c}
\mathrm{z}=0 ; \text { no }- \text { slip boundary condition } \\
\rightarrow \mathrm{u}=\mathrm{u}_{0}, \mathrm{v}=0 \\
\mathrm{z}=\mathrm{h} ; \text { Slip boundary condition } \\
\rightarrow \mathrm{u}=\mathrm{u}_{\mathrm{s}}, \mathrm{v}=\mathrm{v}_{\mathrm{s}}
\end{array}\right.
$$

Which in Eq. 7, $\mathrm{h}_{\mathrm{i}}=\mathrm{h}_{\mathrm{o}}=\mathrm{h}$ and $\tau=\tau_{\mathrm{cx}}$ when $\mathrm{z}=\mathrm{h}$.

$$
\begin{gathered}
\frac{\partial \mathrm{p}}{\partial \mathrm{x}}=\frac{\partial \tau_{\mathrm{xz}}}{\partial \mathrm{z}} \text { and } \\
\tau_{\mathrm{xz}}=\eta \frac{\partial \mathrm{u}}{\partial \mathrm{z}}=\frac{\partial \mathrm{p}}{\partial \mathrm{x}}(\mathrm{z}-\mathrm{h})+\tau_{\mathrm{cx}} \\
\rightarrow \mathrm{u}=\mathrm{u}_{0}+\frac{1}{\eta} \frac{\partial \mathrm{p}}{\partial \mathrm{x}}\left(\frac{\mathrm{z}^{2}}{2}-\mathrm{hz}\right)+\frac{\tau_{\mathrm{cx}}}{\eta} \mathrm{z}
\end{gathered}
$$

Calculating $u_{s}$ from the two component model of Spikes and Granick [29] (Eq. 7) and by applying the boundary conditions (Eq. 10):

$$
\mathrm{z}=\mathrm{h}: \mathrm{u}_{\mathrm{s}}=\mathrm{u}_{0}-\frac{1}{\eta} \frac{\partial \mathrm{p}}{\partial \mathrm{x}}\left(-\frac{\mathrm{h}^{2}}{2}\right)+\frac{\tau_{\mathrm{cx}}}{\eta} \mathrm{h}
$$

The nature of shear stress is always to resist the movement, therefore a sign function is employed in order to consider this effect (Eq. 11):

$$
u_{s}=\frac{\left(u_{0}-\frac{h^{2}}{2 \eta} \frac{\partial p}{\partial x}+\operatorname{sgn}\left(u_{s}\right) \frac{h}{\eta} \tau_{c o}\right)}{1+\frac{h}{b}}
$$

\subsection{Friction calculation}


In these simulations to calculate the coefficient of friction, we have:

$$
\mu=\frac{F_{f}}{W}
$$

where the friction force $\left(\mathrm{F}_{\mathrm{f}}\right)$ generated in a lubricated system is due to shearing the fluid. By integrating interface shear stress over the interface surface area, the friction force $\left(F_{f}\right)$, is obtained:

$$
F_{f}=\int_{0}^{w} \int_{0}^{l} \tau d x d y
$$

where (l) is the length and (w) is the width of surface. In addition, load support (W), is obtained by integration of the pressure:

$$
\mathrm{W}=\int_{0}^{\mathrm{W}} \int_{0}^{\mathrm{l}} \mathrm{pdx} \mathrm{dy}
$$

Therefore, in order to calculate the coefficient of friction, we have:

$$
\mu=\frac{\int_{0}^{w} \int_{0}^{l} \tau d x d y}{\int_{0}^{w} \int_{0}^{l} p d x d y}=\frac{\eta u_{0}}{p h}
$$

In this study the normalized coefficient of friction is employed so as to have a better understanding of boundary slip on textured surfaces on the frictional behaviour of contacts. The normalization of coefficient of friction is possible by dividing the calculated coefficient of friction with a reference coefficient of friction. The reference coefficient of friction can vary in different types of calculations.

$$
\mu^{*}=\frac{\mu}{\mu_{\text {ref }}}
$$

\subsection{Solution}

There are two groups of approaches to solving (Eq. 5), direct solvers and using iterative solvers. Further, it is more common to utilize the de-coupled iteration technique rather than the fully coupled iteration technique. In order to solve this modified Reynolds equation for large-scale lubrication problems, iterative solvers are more applicable because they avoid the storage requirement that a direct solver generally demands. Iterative solvers include nodal iteration and the tri-diagonal matrix algorithm (TMDA) (Patankar [30]). TDMA, also known as the Thomas algorithm, is a simplified form of the Gaussian elimination to solve the tri-diagonal system of equations. It breaks the problem into a series of tri-diagonal sub-problems where any entries outside the tri-diagonal portion are treated as source terms using the previous values. When one solves a two-dimensional problem, the TDMA solution column by column or row by row becomes iterative, and sweeping is done line-by-line and column-bycolumn or row-by-row. Supposing the domain is discretized by $\mathrm{n}_{\text {nodes }}=\mathrm{n}_{\text {col }} \times \mathrm{n}_{\text {row }}$ nodes.

In this case, the computed domain should be divided into a number of control volumes and the modified Reynolds equation will be solved using a TDMA. In the two-dimensional case, it is possible to solve the equations iteratively for the pressure at each grid point using the alternating direction implicit (ADI) method with the tri-diagonal matrix algorithm (TDMA). For more on this the reader is referred to [31]. The numerical solution is presented schematically in the flow chart in appendix $A$.

In their work on the effect of boundary slip combined with textured surfaces, Tauviqirrahman et al. [16, 2527] studied the influence of boundary slip on the loadcarrying capacity and friction force. Although they solved the modified Reynold equation, the effect of cavitation was ignored. Therefore, by considering the effect of cavitation and the boundary slip condition in the Reynolds equation a modified film thickness model is developed in this study.

\section{Results and discussion}

In this section, several numerical simulations have been performed to obtain the influence of boundary slip parameters on the film thickness for textured surfaces. In these calculations, the effect of slip length and critical shear stress $\left(\tau_{c o}\right)$ on hydrodynamic film thickness is investigated. The slip area length in these calculations is equal to $75 \%$ of the contact length; this decision was made based on the study of Tauviqirrahman et al. [28] on the optimization of the boundary slip area.

Table 1. Range of operating parameters.

\begin{tabular}{c|l|c} 
Parameter & Value & Unit \\
\hline Texture depth $\left(\mathrm{T}_{\mathrm{d}}\right)$ & 10 & $\mu \mathrm{m}$ \\
\hline Texture size $(\mathrm{S})$ & 150 & $\mu \mathrm{m}$ \\
\hline Texture Pitch $\left(\mathrm{P}_{\mathrm{x}}\right)$ & 0.5 & - \\
\hline Viscosity & 8 & $\mathrm{mPa}$ \\
\hline Load & 20 & $\mathrm{~N}$ \\
\hline Critical shear stress $\left(\tau_{\mathrm{co}}\right)$ range & $1-2000$ & $\mathrm{kPa}$ \\
\hline Slip area length $\left(\mathrm{L}_{\mathrm{s}} / \mathrm{L}\right)$ & 0.75 & - \\
\hline Contact area & $1 \times 10^{-6}$ & $\mathrm{~m}^{2}$
\end{tabular}


Based on parameters given in table 1, calculations were made in order to find the influence of slip length and critical shear stress on film thickness for textured surfaces, as shown in Fig. 3.

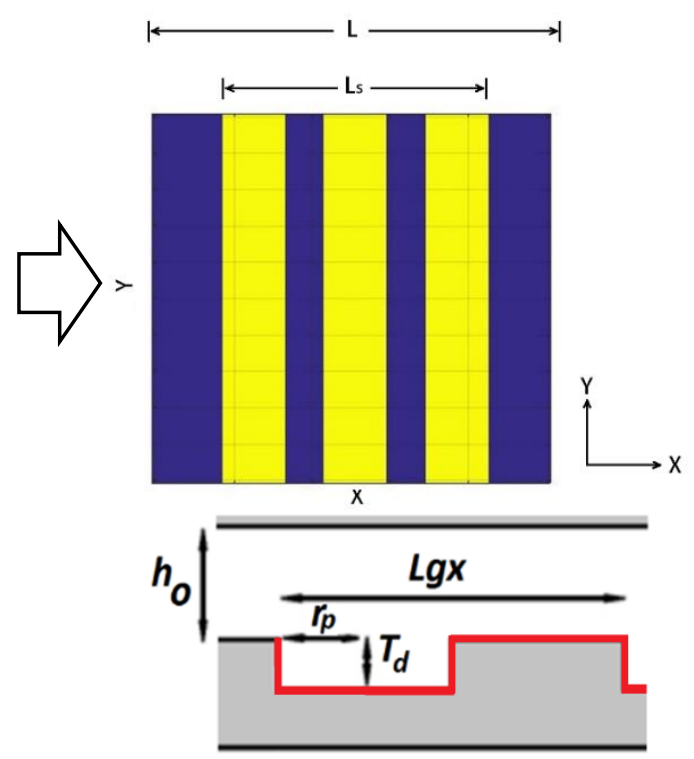

Figure 3. Texture pattern (open groove pattern).

In this study, a rectangular geometry for the texture cell shape is used.

Cavity size: $\mathrm{S}=2 \times \mathrm{r}_{\mathrm{p}}$

Pitch in $x$ direction: $P_{x}=S / L_{g x}$

\subsection{Effect of Slip length (b)}

One of the two main parameters used to characterize wall slip is the slip length. In order to study the influence of this parameter texturing parameters $\left(\mathrm{T}_{\mathrm{d}}, \mathrm{S}\right.$ and $\left.\mathrm{P}_{\mathrm{x}}\right)$ are set as constant and the slip length (b) is varied from 0 to $40 \mu \mathrm{m}$.

As can be seen in Fig. 4, for the textured surface in parallel sliding contact (the wedge effect is absent) increasing the slip length leads to a significant improvement in the film thickness. However, after the slip length passes $20 \mu \mathrm{m}$, the increase in this parameter causes a smaller improvement in film thickness in comparison with the values of slip length smaller than $20 \mu \mathrm{m}$.

The existence of a greater slip length can cause a higher slip velocity on a slippery wall, which results in a larger velocity difference between the fluid velocity on areas with boundary slip and that on non-slip areas. This difference in velocity will cause a pressure gradient, which results in a greater film thickness.

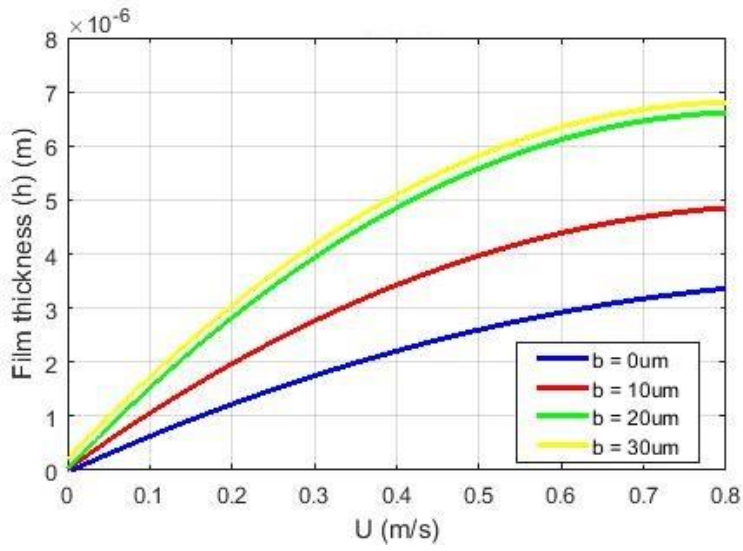

Figure 4. Influence of slip length (b) on film thickness.

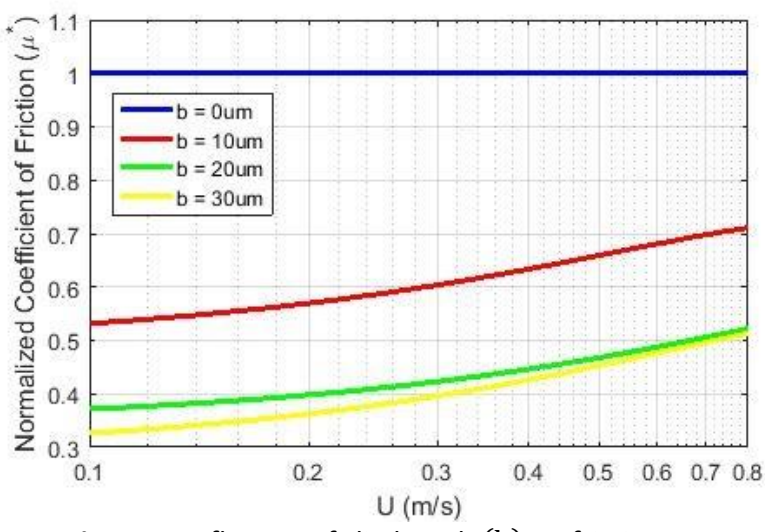

Figure 5. Influence of slip length (b) on friction.

In Fig. 5, the effect of the variation of the slip length on the frictional behaviour of textured surfaces is presented. The coefficient of friction as a function of velocity is calculated for four different values of slip length. The normalized coefficient of friction is calculated by dividing the calculated values by the coefficient of friction for the no-slip condition ( $\mu_{\text {ref }}=$ $\left.\mu_{\mathrm{b}=0}\right)$. This normalization is used to indicate the frictional behaviour of contacts based on different slip lengths.

With respect to the normalized coefficient of friction $\left(\mu^{*}\right)$, the increase in the slip length leads to a decrease in this parameter $\left(\mu^{*}\right)$. It can also be observed that if the slip length decreases to zero, $\mu^{*}$ leads to its no-slip value. It is shown that the rate of friction drop is higher from $b=0 \mu \mathrm{m}$ to $\mathrm{b}=20 \mu \mathrm{m}$ than from $\mathrm{b}=$ $20 \mu \mathrm{m}$ to $\mathrm{b}=30 \mu \mathrm{m}$.

\subsection{Effect of critical shear stress $\left(\boldsymbol{\tau}_{\text {co }}\right)$}

The focus of this subsection is to show the effect of the critical shear stress on the frictional behaviour of parallel sliding textured surfaces. Therefore, in these calculations the critical shear stress of a partially 
slip/textured surface is changed between [1kPa $2 \mathrm{MPa}$ ] to observe the influence of this parameter over the film thickness and friction. As can be observed from Fig. 6, the critical shear stress has a significant effect on the predicted film thickness. The greatest film thickness is obtained when employing a surface with a low critical shear stress $\left(\tau_{\text {co }}=1 \mathrm{kPa}\right.$ in this case). For a low critical shear stress, more lubricant can enter the contact, thereby increasing the hydrodynamic pressure.

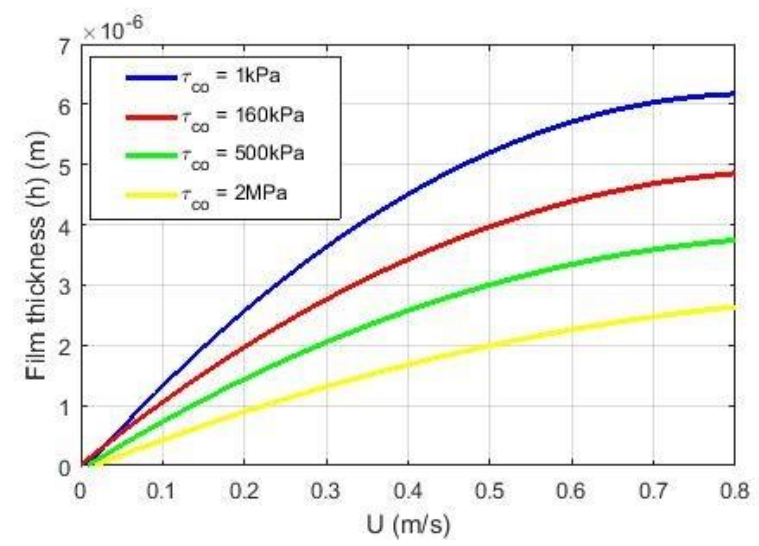

Figure 6. Influence of critical shear stress $\left(\tau_{\mathrm{co}}\right)$ on film thickness.

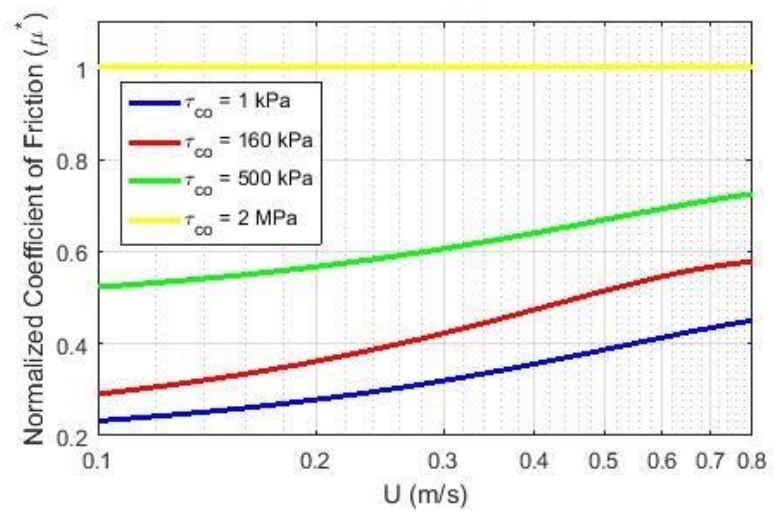

Figure 7. Influence of critical shear stress $\left(\tau_{\mathrm{co}}\right)$ on friction.

Increasing the critical shear stress causes the shear stress between the lubricant and wall to increase at the slippery surface, resulting in more resistance of the fluid flow in the contact area and consequently a lower slip velocity and lower load-carrying capacity. Therefore, increasing the critical shear stress reduces the film thickness.

For friction as a function of the critical shear stress $\left(\tau_{\text {co }}\right)$ as shown in Fig. 7 , the normalized coefficient of friction $\mu^{*}$ is used. To normalize the coefficient of friction, the highest coefficient of friction calculated, i.e. when $\tau_{\text {co }}=2 \mathrm{MPa}$ is taken as the reference coefficient of friction $\left(\mu_{\text {ref }}\right)$. This value of coefficient of friction is equal to the value of coefficient of friction when there is no boundary slip. From Fig. 7 it is clear that by increasing the critical shear stress $\left(\tau_{\text {co }}\right)$ the coefficient of friction of textured surfaces increases; in other words, the lowest normalized coefficient of friction is achievable when $\tau_{\text {co }}$ is close to zero. Therefore, when the critical shear stress value is reduced to a very low level, i.e. close to zero, the boundary slip leads to a significant improvement in friction reduction.

\section{Discussion}

The results of the numerical calculations showed that the existence of boundary slip leads to an increase in the film thickness and a reduction in the coefficient of friction. With respect to the film thickness, a textured surface with boundary slip is superior to merely a textured surface.

The existence of boundary slip and slip velocity across the surfaces results in a higher average inlet velocity of lubricant into the contact area. This increase in average velocity causes the growth in fluid flow in the contact region and, as a result, a higher amount of lubricant in that region, i.e. a larger separation between two surfaces. Therefore, by increasing the slip velocity it is possible to thicken the lubricant film in the contact region.

The slip length can control the fluid flow and, as shown, the film thickness in the contact region. It is worth considering that in this study the full film lubrication $(\mathrm{HL})$ is investigated, and that in the $\mathrm{HL}$ regime a higher value for the slip length results in a lower coefficient of friction because of a thicker film (see Eq. 13). Therefore, if the reduction of friction in the full film lubrication situation is the goal, employing boundary slip is beneficial when surface texturing is present.

\section{Conclusions}

The aim of the investigation was to develop a numerical model in order to have a better understanding of film thickness for textured surfaces with boundary slip conditions. The model is based on a numerical algorithm based on the modified Reynolds equation with the Elrod cavitation algorithm formulation. The equations were made discrete using the finite difference method and solved using the TDMA iterative method. In addition, in order to study the frictional behaviour of parallel sliding contact when the texturing 
and boundary slip are applied, the normalized coefficient of friction is used.

From the study on the influence of slip length on the film thickness and friction it is possible to observe that a thicker lubricant film is achievable when the slip length increases, due to a higher slip velocity. The positive effect that increasing the slip length has on film formation is less significant when the value of this parameter is greater than $20 \mu \mathrm{m}$. With respect to the normalized coefficient of friction $\left(\mu^{*}\right)$, the increase in the slip length leads to a decrease in this parameter $\left(\mu^{*}\right)$.

The influence of the critical shear stress on film thickness and friction was also studied and based on the calculations it is observed that when the boundary slip is present, the highest film thickness is achievable at the lowest values of the critical shear strength. Therefore, by increasing the critical shear stress $\left(\tau_{\mathrm{co}}\right)$, the coefficient of friction in textured surfaces increases and the lowest normalized coefficient of friction is achievable when $\tau_{\text {co }}$ is close to zero.

Based on this study the optimum value for slip length (b) is around $20 \mu \mathrm{m}$. In the case of the critical shear stress $\left(\tau_{\mathrm{co}}\right)$ the lowest coefficient of friction is achieved when $\tau_{\mathrm{co}}=0 \mathrm{kPa}$. However, the rate of friction reduction between $\tau_{\text {co }}=1 \mathrm{kPa}$ and $\tau_{\text {co }}=160 \mathrm{kPa}$ is smaller than the rate friction reduction between $\tau_{\text {co }}=$ $160 \mathrm{kPa}$ and $\tau_{\text {co }}=250 \mathrm{kPa}$, so a critical shear stress around $160 \mathrm{kPa}$ can be beneficial to decreasing the friction in lubricated contacts.

\section{References}

[1] Spikes, H., and Granick, S., 2003, "Equation for Slip of Simple Liquids at Smooth Solid Surfaces," Langmuir, 19(12), pp. 5065-5071.

[2] Goldstein, S., 1938, "Modern developments in fluid mechanics," Vol. II, pp. 601-607.

[3] Vinogradova, O. I., 1999, "Slippage of water over hydrophobic surfaces," International Journal of Mineral Processing, 56(1-4), pp. 31-60.

[4] Debye, P., and Cleland, R. L., 1959, "Flow of liquid hydrocarbons in porous vycor," Journal of Applied Physics, 30(6), pp. 843-849.

[5] Ruckenstein, E., and Rajora, P., 1983, "On the noslip boundary condition of hydrodynamics," Journal of Colloid And Interface Science, 96(2), pp. 488-491.

[6] Churaev, N. V., Sobolev, V. D., and Somov, A. N., 1984, "Slippage of liquids over lyophobic solid surfaces," Journal of Colloid And Interface Science, 97(2), pp. 574-581.

[7] Pit, R., Hervet, H., and Leger, L., 2000, "Direct experimental evidence of slip in hexadecane: solid interfaces," Physical review letters, 85(5), p. 980.

[8] Pit, R., Hervet, H., and Léger, L., 1999, "Friction and slip of a simple liquid at a solid surface," Tribol Lett, 7(2-3), pp. 147-152.

[9] Zhu, Y., and Granick, S., 2002, "Limits of the hydrodynamic no-slip boundary condition," Phys Rev Lett, 88(10), p. 106102.

[10] Zhu, Y., and Granick, S., 2001, "Rate-Dependent Slip of Newtonian Liquid at Smooth Surfaces," Physical Review Letters, 87(9), p. 096105.

[11] Boehnke, U. C., Remmler, T., Motschmann, H., Wurlitzer, S., and Hauwede, J., 1999, "Partial air wetting on solvophobic surfaces in polar liquids," Journal of Colloid and Interface Science, 211(2), pp. 243-251.

[12] Zhu, Y., and Granick, S., 2001, "Rate-dependent slip of Newtonian liquid at smooth surfaces," Physical Review Letters, 87(9), pp. 961051961054.

[13] Baudry, J., Charlaix, E., Tonck, A., and Mazuyer, D., 2001, "Experimental Evidence for a Large Slip Effect at a Nonwetting Fluid-Solid Interface," Langmuir, 17(17), pp. 5232-5236.

[14] Spikes, H. A., 2003, "The half-wetted bearing. Part 1: extended Reynolds equation," Proceedings of the Institution of Mechanical Engineers, Part J: Journal of Engineering Tribology, 217(1), pp. 1-14.

[15] Spikes, H. A., 2003, "The half-wetted bearing. Part 2: potential application in low load contacts," Proceedings of the Institution of Mechanical Engineers, Part J: Journal of Engineering Tribology, 217(1), pp. 15-26.

[16] Tauviqirrahman, M., Ismail, R., Jamari, and Schipper, D. J., 2013, "Optimization of the complex slip surface and its effect on the hydrodynamic performance of two-dimensional lubricated contacts," Computers \& Fluids, 79(0), pp. 27-43.

[17] Ma, G. J., Wu, C. W., and Zhou, P., 2007, "Wall slip and hydrodynamics of two-dimensional journal bearing," Tribology International, 40(7), pp. 10561066.

[18] Schowalter, W. R., 1988, "The behavior of complex fluids at solid boundaries," Journal of Non-Newtonian Fluid Mechanics, 29(C), pp. 25-36.

[19] Léger, L., Raphaël, E., and Hervet, H., 1999, "Surface-anchored polymer chains: Their role in adhesion and friction," Adv. Polym. Sci., 138, pp. 185-225. 
[20] Denn, M. M., 2001, "Extrusion instabilities and wall slip," Annual Review of Fluid Mechanics, pp. 265-287.

[21] Wang, S. Q., 1999, "Molecular transitions and dynamics at melt/wall interfaces: Origins of flow instabilities and wall slip," Adv. Polym. Sci., 138.

[22] Craig, V. S. J., Neto, C., and Williams, D. R. M., 2001, "Shear-dependent boundary slip in an aqueous Newtonian liquid," Physical Review Letters, 87(5), pp. 054504/054501054504/054504.

[23] Bonaccurso, E., Kappl, M., and Butt, H. J., 2002, "Hydrodynamic force measurements: Boundary slip of water on hydrophilic surfaces and electrokinetic effects," Physical Review Letters, 88(7), pp. 761031-761034.

[24] Zhu, Y., and Granick, S., 2002, "No-slip boundary condition switches to partial slip when fluid contains surfactant," Langmuir, 18(26), pp. 1005810063.

[25] Tauviqirrahman, M., 2013, "Lubricated MEMS: effect of boundary slippage and texturing, PhD Thesis," PhD PhD Thesis, Unversity of Twente, Enschede.

[26] Tauviqirrahman, M., Ismail, R., Jamari, J., and Schipper, D. J., 2013, "Combined effect of texturing and boundary slippage in lubricated sliding contacts," Tribology International, 66(0), pp. 274-281.

[27] Tauviqirrahman, M., Ismail, R., Jamari, J., and Schipper, D. J., 2013, "Computational analysis of the lubricated-sliding contact with artificial slip boundary," International Journal of Applied Mathematics and Statistics, 35(5), pp. 67-80.

[28] Tauviqirrahman, M., Ismail, R., Jamari, J., and Schipper, D. J., 2013, "A study of surface texturing and boundary slip on improving the load support of lubricated parallel sliding contacts," Acta mechanica, 224(2), pp. 365-381.

[29] Choo, J. H., Spikes, H. A., Ratoi, M., Glovnea, R., and Forrest, A., 2007, "Friction reduction in lowload hydrodynamic lubrication with a hydrophobic surface," Tribology International, 40(2), pp. 154-159.

[30] Patankar, S. V., 1980, Numerical heat transfer and fluid flow / Suhas V. Patankar, Hemisphere Pub. Corp. ; McGraw-Hill, Washington : New York.

[31] Bijani, D., Deladi, L. E., and Schipper, D. J., 2016, "The Influence of Surface Texturing on the Film Thickness in Parallel Sliding Surfaces," 20th International Colloquium Tribology: Technische Akademie Esslingen, p. 12. 


\title{
Paper F
}

\section{An experimental study on the influence of surface texturing in flat on flat lubricated contacts}

\author{
Dariush Bijani, M.B. de Rooij, G.R.B.E. Römer, E.L. Deladi and D.J. Schipper
}

(Article in press)

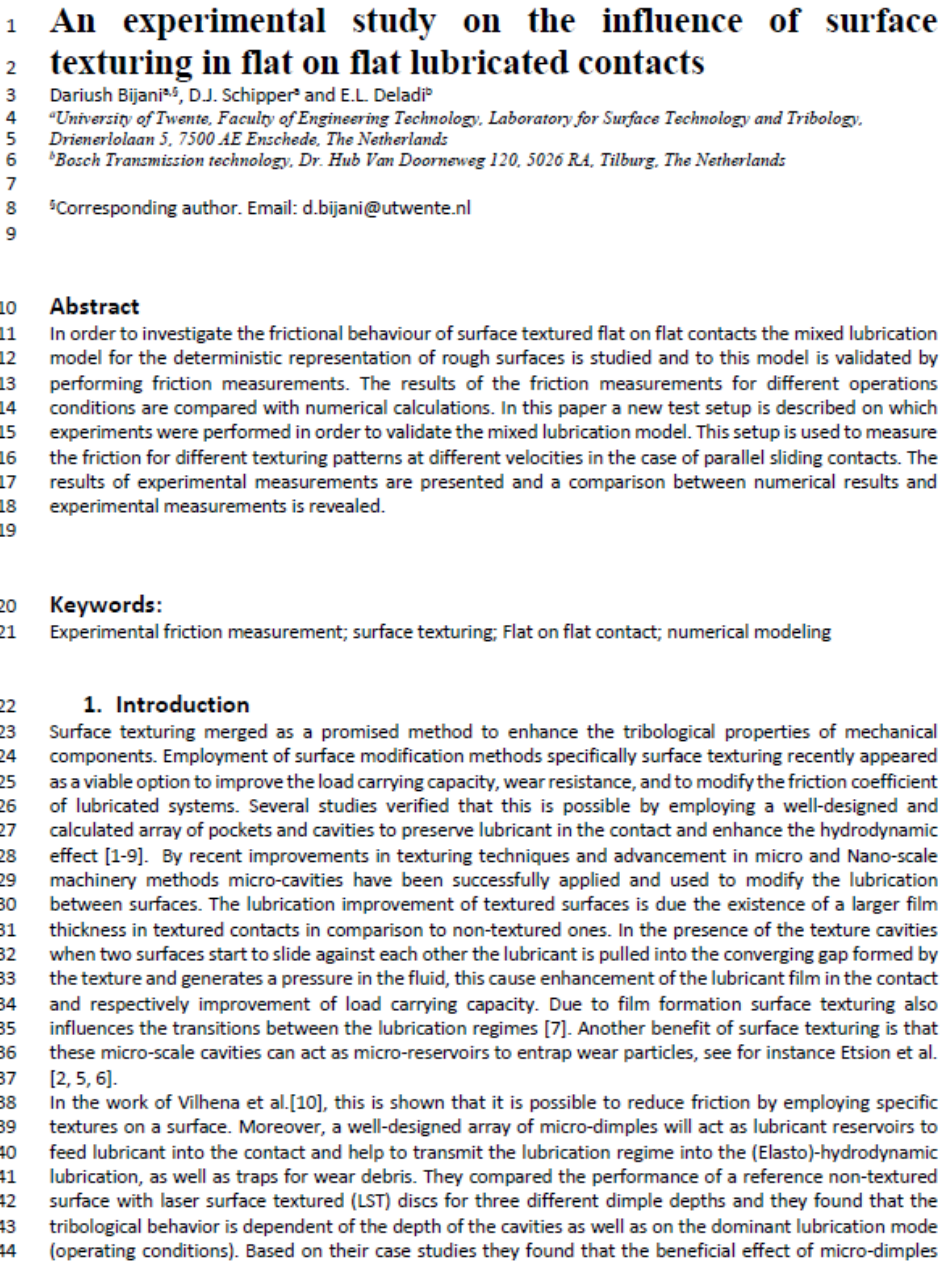

An experimental study on the influence of surface

Wniversity of Twente, Faculty of Engineering Tech olo

Bosch Transmission technology. Dr. Hub Van Doorneweg 120, 5026 RA, Tilburg. The Netherland

${ }^{5}$ Corresponding author. Email: d.bijani@utwente.n

Abstract

In order to investigate the frictional behaviour of surface textured flat on flat contacts the mixed lubrication model for the deterministic representation of rough surfaces is studied and to this model is validated by results of experimental measurements are presented and a comparison between numerical results and

Keywords:

Experimental friction measurement; surface texturing; Flat on flat contact; numerical modeling

1. Introduction d Nano-scale p formed by the texture and generates a pressure in the fluid, this cause enhancement of the lubricant film in the contact influences the transitions between the lubrication regimes [7]. Another benefit of surface texturing is that these micro-scale cavities can act as micro-reservoirs to entrap wear particles, see for instance Etsion et al.

In the work of Vilhena et al. [10], this is shown that it is possible to reduce friction by employing specific textures on a surface. Moreover, a well-designed array of micro-dimples will act as lubricant reservoirs to surface with laser surface textured (LST) discs for three different dimple depths and they found that the tribological behavior is dependent of the depth of the cavities as well as on the dominant lubrication mode
\end{abstract}





\title{
An experimental study on the influence of surface texturing in flat on flat lubricated contacts
}

\author{
Dariush Bijani, ${ }^{a}$, M.B. de Rooij ${ }^{b}$, G.R.B.E. Römer ${ }^{c}$, E.L. Deladid and D.J. Schipper ${ }^{b}$ \\ a Materials Innovation Institute (M2i), P.O. Box 5008, 2600 GA Delft, the Netherland, \\ bUniversity of Twente, Faculty of Engineering Technology, Laboratory for Surface Technology and Tribology, \\ Drienerlolaan 5, 7500 AE Enschede, The Netherlands \\ 'University of Twente,, Faculty of Engineering Technology, Laser Processing, Drienerlolaan 5, 7500 AE Enschede, The \\ Netherlands

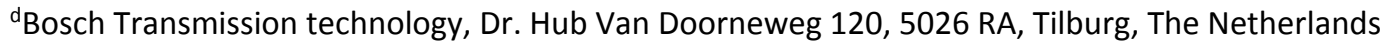

${ }^{\S}$ Corresponding author. Email: d.bijani@utwente.nl

\begin{abstract}
In order to investigate the frictional behaviour of surface textured flat on flat contacts the mixed lubrication model for the deterministic representation of rough surfaces is studied and this model is validated by performing friction measurements. The results of the friction measurements for different operating conditions are compared with numerical calculations. In this paper a new test setup is described on which experiments were performed in order to validate the mixed lubrication model. This setup is used to measure the friction for different texturing patterns at different velocities in the case of parallel sliding contacts. The results of the experimental measurements are presented and a comparison between the numerical results and the experimental measurements is explained.
\end{abstract}

\section{Keywords:}

Experimental friction measurement; surface texturing; Flat on flat contact; numerical modeling

\section{Introduction}

Surface texturing emerged as a promising method of enhancing the tribological properties of mechanical components. Employment of surface modification methods, and specifically surface texturing recently appeared as a viable option to improve the loadcarrying capacity wear resistance, and to modify the friction coefficient of lubricated systems. Several studies verified that this is possible by employing a welldesigned and calculated array of pockets and cavities to preserve lubricant in the contact and enhance the hydrodynamic effect [1-9]. Thanks to recent improvements in texturing techniques and advancement in micro and nano-scale machinery methods micro-cavities have been successfully applied and used to modify the lubrication between surfaces. The lubrication improvement of textured surfaces is due to the existence of a greater film thickness in textured contacts than in non-textured ones. In the presence of the texture cavities when two surfaces start to slide against each other, the lubricant is pulled into the converging gap formed by the texture and generates a pressure in the fluid, causing enhancement of the lubricant film in the contact and improvement of the load-carrying capacity. Due to film formation, surface texturing also influences the transitions between the lubrication regimes [7]. Another benefit of surface texturing is that these micro-scale cavities can act as micro-reservoirs to entrap wear particles, see for instance Etsion et al. [2, 5, 6].

In the work of Vilhena et al.[10], it is shown that it is possible to reduce friction by employing specific textures on a surface. Moreover, a well-designed array of micro-dimples will act as lubricant reservoirs to feed lubricant into the contact and help to transmit the lubrication regime into the (Elasto)-hydrodynamic 
lubrication, as well as traps for wear debris. They compared the performance of a reference nontextured surface with laser surface textured (LST) discs for three different dimple depths and found that the tribological behaviour is dependent on the depth of the cavities as well as on the dominant lubrication mode (operating conditions). Based on their case studies they found that the beneficial effect of micro-dimples becomes more significant with an increase in dimple depth as well as for higher sliding speeds. They conclude that, at higher sliding speeds, micro-dimples on a sliding surface can act as micro pressure chambers, which provide hydrodynamic action.

Further, the influence of surface texturing and its geometrical characteristics on lubrication and the transition from mixed to hydrodynamic lubrication is studied by Galda et al. [11]. A block on ring machine is used in their experimental investigation. Tests were conducted at increasing sliding speed in the range of $0.08-0.69 \mathrm{~m} . \mathrm{s}^{-1}$ under conformal contact conditions with unidirectional sliding. They showed that by employing the proper shape and dimensions the friction characteristic of the sliding pairs could be improved with comparison to non-textured surfaces. In their work they conclude that, the spherical oil pockets and the dimples of long drop shape are more efficient in minimizing the coefficient of friction than pits of short drop shape. Moreover, in their measurements the influence of texture density is studied with respect to lubrication regime transitions. In another study Galda et al. [12] investigated experimentally the possibility of friction reduction on surface textured journal bearings during the shut-down period. Measurements were performed on journal bearings with oil pockets in the journal surface mating with a smooth bearing and the surface texture on the bearing surface co-acting with a standard smooth journal. To illustrate the capability of a considerable friction reduction the friction torque values of the examined sliding pairs were compared as a function of the rotational speed and Hersey number. They found that, during shut-down, the textured journal bearings remained in hydrodynamic lubrication for longer and the lubrication regime changes to mixed lubrication at lower speeds than the smooth journal bearing. Within these measurements at a low Hersey number considerably smaller friction coefficient values are obtained for the textured series than for the smooth surfaces, but the friction of the textured series tended to increase at a higher Hersey number. They conclude that to employ the texture in contacts for specific applications the optimum texture dimensions have to be determined.

In order to investigate the effects of geometrical characteristics and operating conditions Zhong et al. [13] examined the tribological performance of hexagonal textures of two orientations $\left(\theta=0^{\circ}\right.$ and $\left.90^{\circ}\right)$. They developed a numerical model and fabricated the corresponding specimens by laser texturing to study these specimens both theoretically and experimentally. Overall, their experiments have verified the trends identified from the simulations that the hexagonal textured surface with an area density of $25 \%$ can reduce the friction coefficient up to $41 \%$, as compared to a smooth surface. In this study, the coefficient of friction for chevron and groove textured surfaces are measured. Further, the measurement results are compared with results from the numerical calculations and simulation of frictional behaviour of flat on flat contacts. In appendix A, the method and algorithm to calculate the coefficient of friction in flat on flat contact are explained.

\section{Measurement setup}

To measure friction of lubricated plan parallel sliding textured surfaces, the test setup and method is used as described in this section are used as to validate the numerical model that is described in Appendix $A$,

To measure the friction in a flat on flat situation, the most challenging obstacle is to prepare samples with respect to flatness and to align these flat surfaces. The existence of any misalignment or surface waviness of the samples will cause a measurement error. The operating conditions of these measurements are presented in Table 1.

Table 1. Measurement conditions.

\begin{tabular}{l|l} 
Normal load & $5,10 \mathrm{~N}$ \\
\hline Velocities & $0.05,0.1,0.2,0.3,0.4,0.5 \mathrm{~m} . \mathrm{s}^{-1}$ \\
\hline Lubricant type & NS3 Shell \\
\hline Lubricant & $32 \mathrm{mPa} . \mathrm{s}$ \\
viscosity &
\end{tabular}

\subsection{Pin-on-Disk}

The experiments were conducted in a pin-on-disk tribotester (CSEM), see Fig. 4. The tested specimens are flat (textured) steel blocks (the pin) pressed against 
rotating steel disks held in a cup filled with the lubricant used. The samples were prepared in the workshop of the University of Twente; the laser texturing of the samples was conducted at Philips and at the laser lab of the University of Twente.

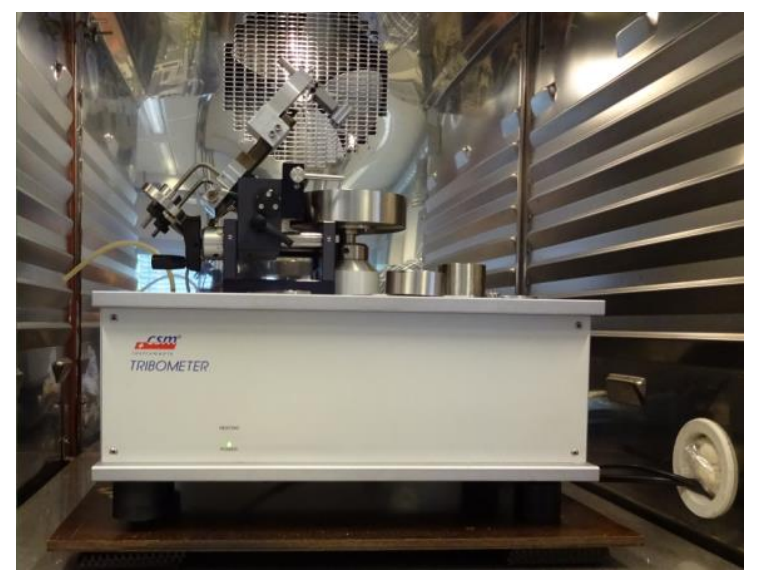

(a)

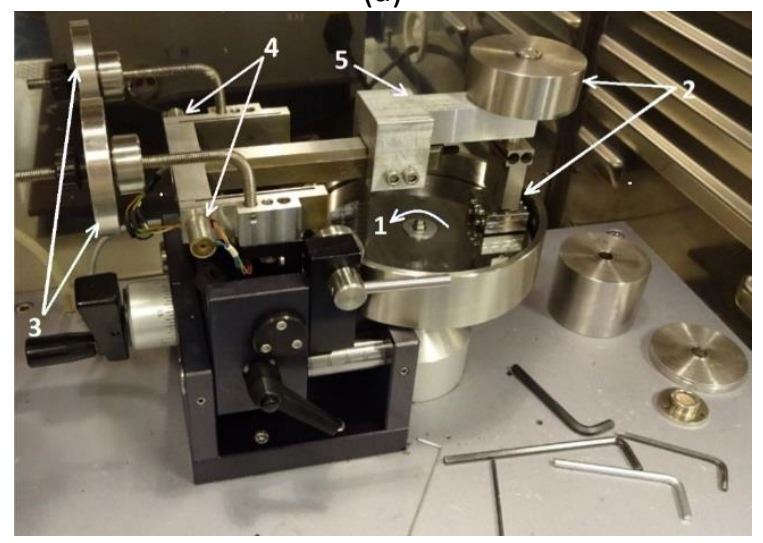

(b)

Figure 1. (a) Pin-on-disk setup. (b) Detail of the setup.

The set-up is most frequently used to test the friction in a ball-on-disk configuration (point contact). Since we are interested in measuring friction in a flat-on-flat configuration, the set-up has been modified to include a special holder to compensate for alignment issues. Further, a larger disk is used to make the contact inlet lubricant flow as uniform as possible (using the available set-up).

Fig. 4.b shows the setup in more detail. The numbered parts are:

1. Smooth, stainless steel rotating disk,

2. Sample holder with a dead weight on top,

3. Counter weights to balance the mass of the arm (5), sample and sample-holder (2),

4. "Friction" sensors,

5. Arm extension.
The arm is mounted to the frame via flexures near the sensors. As the disk rotates the friction force will cause the arm to deflect. This deflection is measured and converted into a friction force. The coefficient of friction is obtained by dividing the friction force by the normal load (for the load caused by the weight) using software provided by CSEM Instruments.

\subsection{Sample holder}

In order to have an aligned flat-flat contact it is necessary to have a self-adjustment mechanism for the samples. By pivoting in two directions (parallel and perpendicular to the sliding direction) around the centre point of contact, it is possible for the sample to align. Therefore, by mounting such a sample holder into the pin-on-disk machine the sample can self-adjust in parallel and perpendicular direction to the sliding direction.

The schematic detail of the specimen holder is presented in Fig. 2. As can be seen, the specimen holder has three main parts. From top to bottom of the holder, the first part (1) is the hinge, the second part (2) is the elastic plates and the third part (3) is the specimen grip. The purpose of the hinge is to adjust the sample with respect to misalignments, irregularities on the disk in perpendicular direction (1) and in the moving direction (2) as shown in Fig. 2.

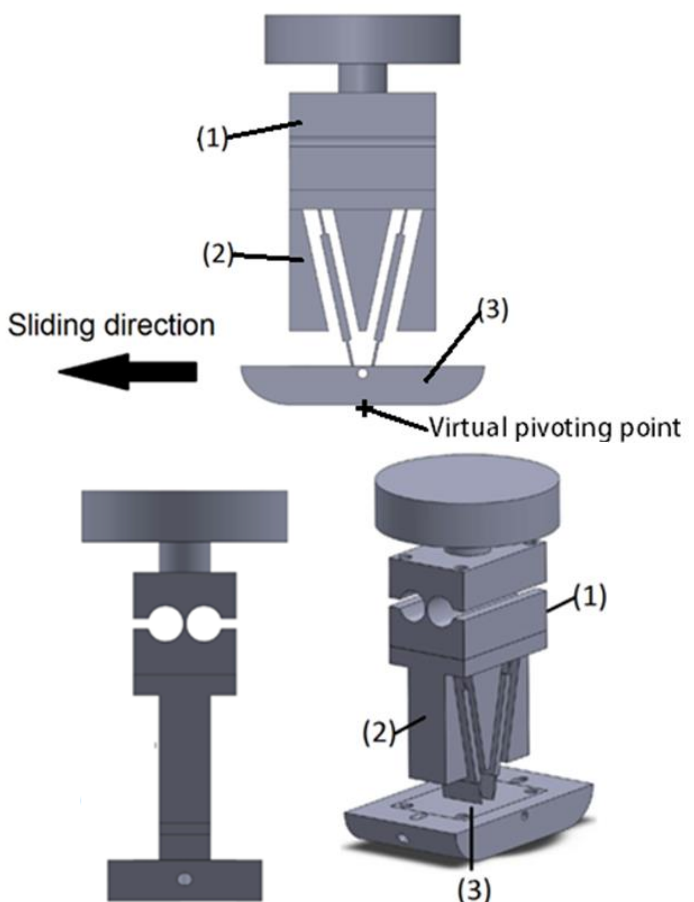

Figure 2. Schematic illustration of the designed specimen holder. 
In Fig. 2, details of the designed specimen holder is shown as well as the mounted specimen holder in the pin-on-disk tribometer.

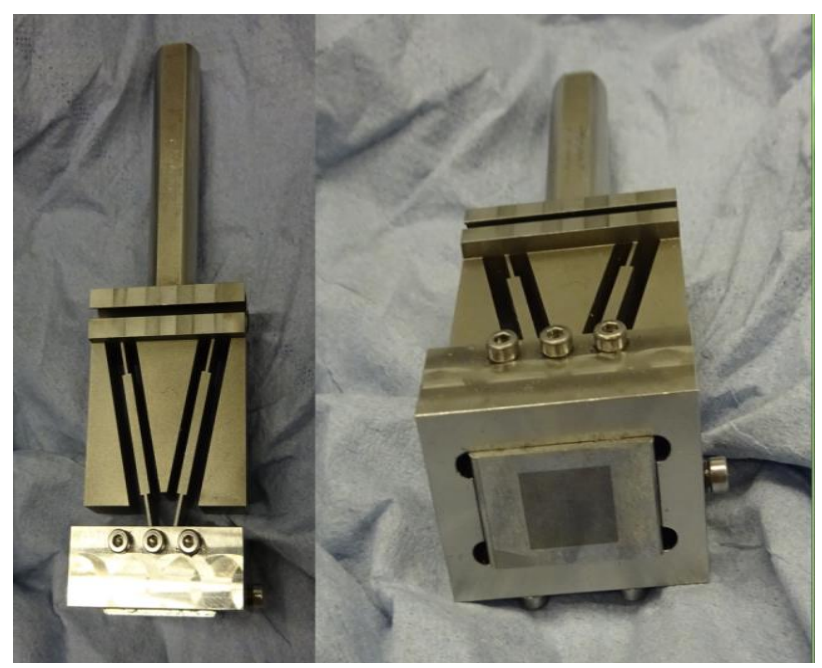

(a)

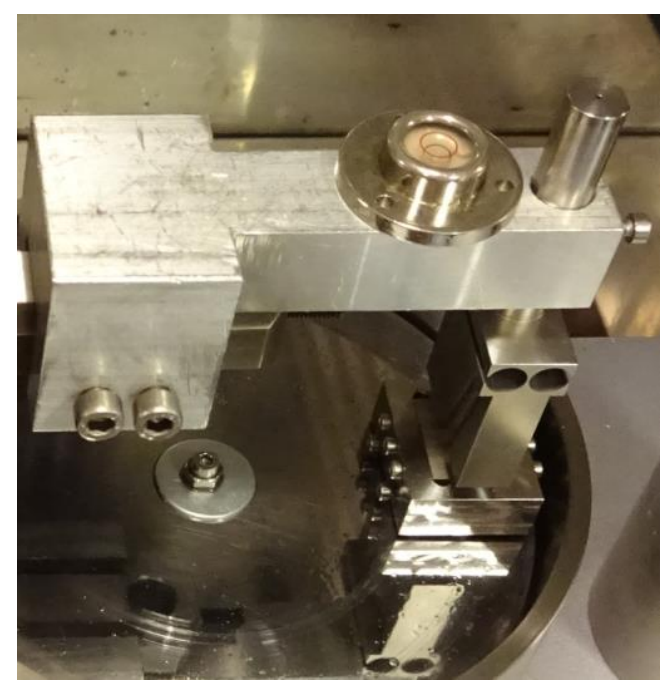

(b)

Figure 3. a) Details of the sample holder, b) the mounted specimen holder.

\subsection{Samples}

As an example in Fig. 4, the topography of a chevron cell measured with the confocal microscope is presented.
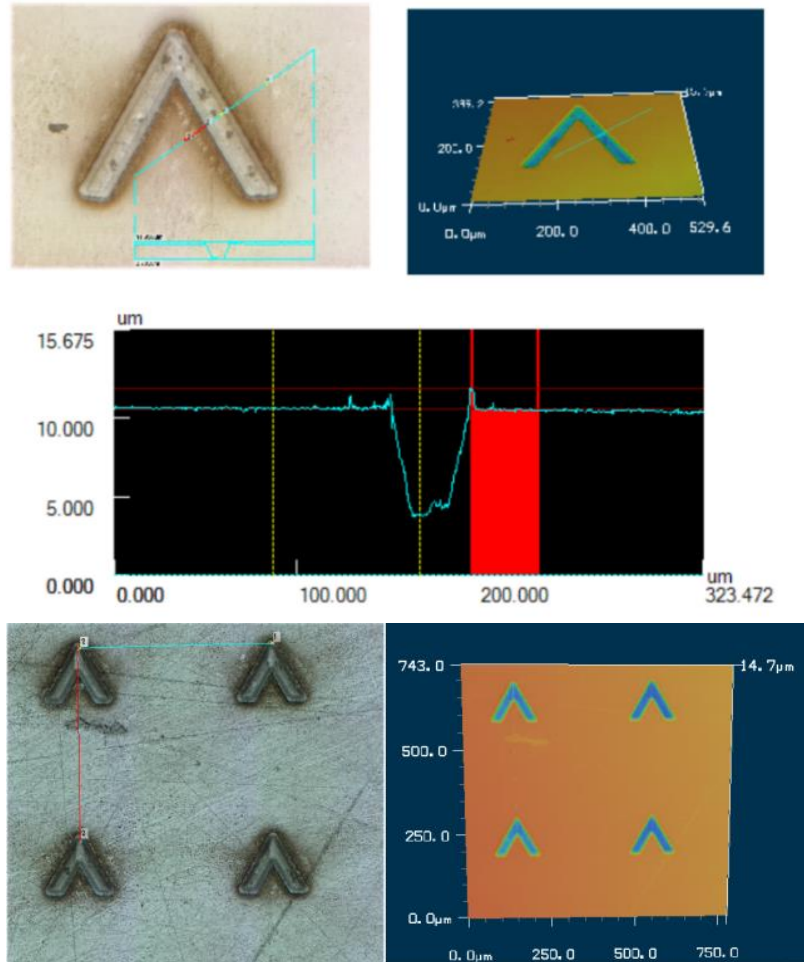

Figure 4. Confocal microscope images of LST chevron pattern.

A schematic illustration of textured area of the samples shown in Fig. 5.

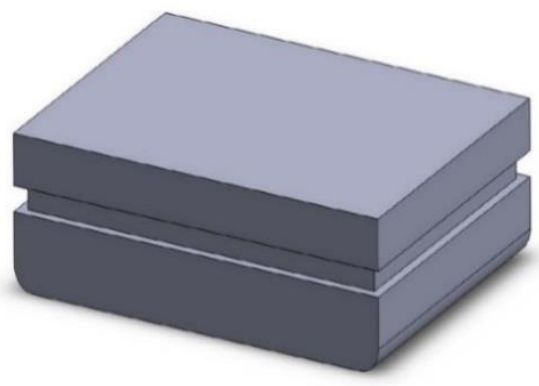

(a)

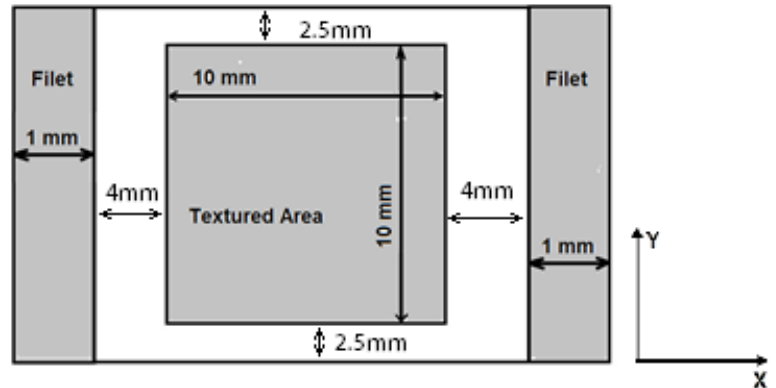

(b) 


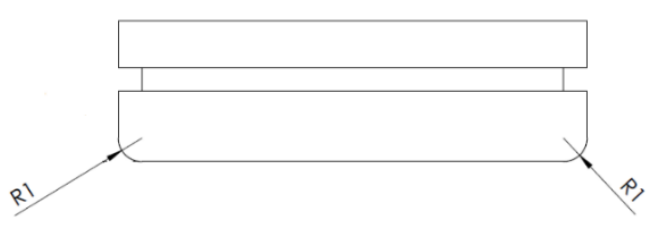

(c)

Figure 5. (a) 3D Schematic illustration of sample, (b) Schematic illustration and dimensions of the textured area on bottom surface of the samples, (c) geometry inlet and outlet specimen.

To stream a uniform lubricant film into the flat on flat contact area, the inlet and outlet fillets with radius of $1 \mathrm{~mm}$ are designed and the symmetrical design of samples and use of outlet fillet makes the outlet flow uniform as well. The textured area is located on the flat area of surface with $4 \mathrm{~mm}$ distance from the inlet and outlet fillets to avoid the possible influence of wedge effect caused by the curvature of fillets.

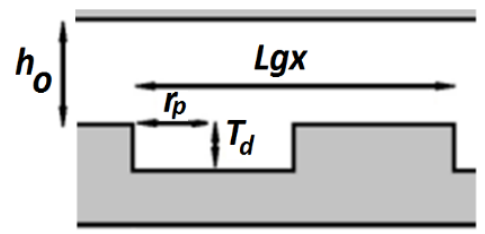

Figure 6. Schematic illustration of the cavity profile.

In Fig. 6 the parameters characterizing chevron and groove patterns are presented. The chevron pattern is defined by two similar equilateral triangles of different sizes and the centre of the unit cell coincides with the midpoint of the altitude line of the chevron shape, see also [22]. In Figure 6, $r_{\mathrm{p}}$ is the characteristic radius for chevron patterns, which is the half width of groove as well. The cross-sectional profiles of these two patterns are rectangular (Fig. 6).

Different depths, pitches and sizes of texturing are selected as presented in Table 2:

Table 2. Textured samples characteristics.

\begin{tabular}{|c|c|c|c|c|c|c|}
\hline \multirow{3}{*}{$\begin{array}{l}\text { Pitch } \\
\text { Depth }(\mu m)\end{array}$} & \multirow{2}{*}{\begin{tabular}{|c|} 
Chevrons \\
0.4 \\
\end{tabular}} & \multicolumn{5}{|c|}{ Grooves } \\
\hline & & \multicolumn{4}{|c|}{0.4} & 0.45 \\
\hline & 10 & 5 & \multicolumn{3}{|c|}{10} & 10 \\
\hline $\begin{array}{l}\text { Cavity length } \\
\text { in } X(\mu m)\end{array}$ & 100 & $\begin{array}{c}10 \\
0\end{array}$ & 50 & 100 & 200 & 200 \\
\hline $\begin{array}{l}\text { Cavity length } \\
\text { in } Y(\mu \mathrm{m})\end{array}$ & 100 & 10 & 10 & 10 & 10 & 10 \\
\hline $\begin{array}{l}\text { Number of } \\
\text { arrays in } X\end{array}$ & 40 & 40 & 80 & 40 & 20 & 23 \\
\hline $\begin{array}{l}\text { Number of } \\
\text { arrays in } Y\end{array}$ & 40 & 1 & 1 & 1 & 1 & 1 \\
\hline
\end{tabular}

\subsection{Confocal Microscope}

Surface topography analyses have been performed using a Keyence Colour 3D LASER Scanning Microscope which uses a violet $\operatorname{LASER} \lambda=388 \mathrm{~nm}$. The control unit is a Keyence VK- $9700 \mathrm{~K}$ combined with a Keyence VK9710K measurement unit, shown in Fig. 8.

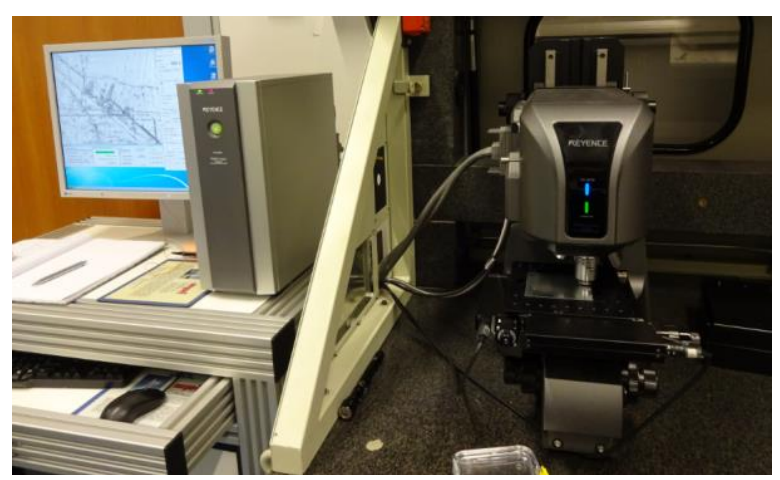

Figure 7. Keyence VK-9700K confocal microscope with a VK9710K measurement unit.

\section{Results and discussion}

In this section the results of several friction experiments that were performed are presented.

\subsection{Non-textured surface}

Firstly, initial measurements using a non-textured sample were performed. In the case of non-textured samples no pressure is built up and therefore there is no lubricant film formation. Further, based on the study of the coefficient of friction and the effect of surface texturing on it, when there is no film formation the coefficient of friction is expected to be equal to the coefficient of friction under boundary lubrication conditions.

If a misalignment exists, it is possible for the contact to operate in the boundary lubrication regime in one direction, whereas in the opposite direction (because of the existence of a wedge effect) the lubrication will be in a different lubrication regime and the coefficient of friction will be lower. For these reasons, friction is measured for several velocities and to check the reliability of specimen holder these measurements were also performed in the opposite sliding direction. In Fig. 8, the Stribeck curve for the non-textured sample in both sliding directions is presented. The measurements are performed for four different velocities, and at least three measurements were executed for every value of a velocity. The error bars in these results show the maximum and minimum values 
measured and the line through the error bar shows the average measured value of the coefficient of friction as a function of velocity.

In Fig. 8, it is shown that for the non-textured samples the coefficient of friction is constant, i.e. no hydrodynamic action is found. The level for the coefficient of friction is in the range of $0.105-0.115$, and the mean value is approximately 0.11 . The friction in both directions is approximately the same showing that the sample holder performs well.

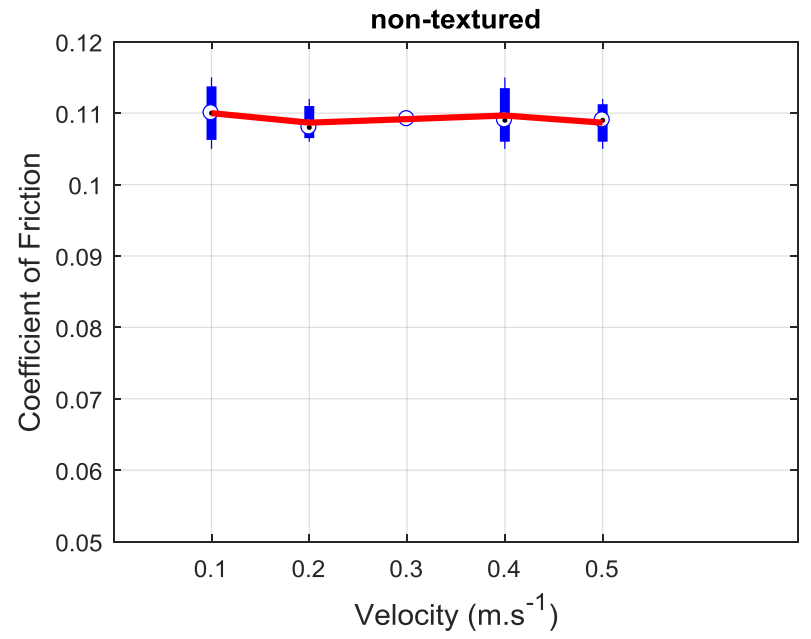

Figure 8. Coefficient of friction as a function of velocity for non-textured flat-flat contact $\left(F_{T}=5 \mathrm{~N}\right.$ and $\eta=32 \mathrm{mPa}$.s $)$.

\subsection{Measurement results with textured surfaces}

After the validation of the sample holder's reliability, friction measurements using samples with different texturing shapes and dimensions were performed. Several measurements for grooved samples (Fig. 9) and one chevron sample (Fig. 10) were performed; the data extracted from these measurements is used to examine the numerical predictions with respect to the influence of texturing parameters, see section 3.3 et seq. The influence of cavity depth, size and pitch on friction was the focus of these measurements.

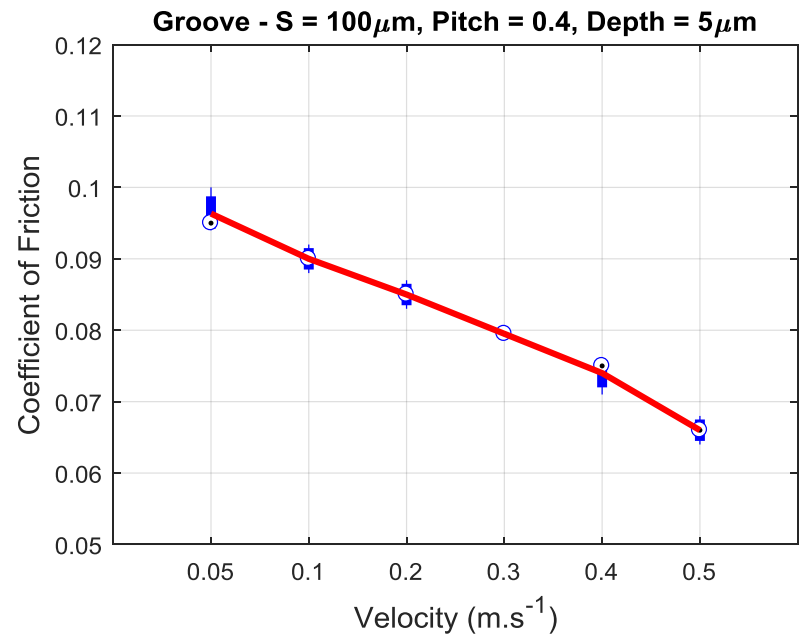

(a)

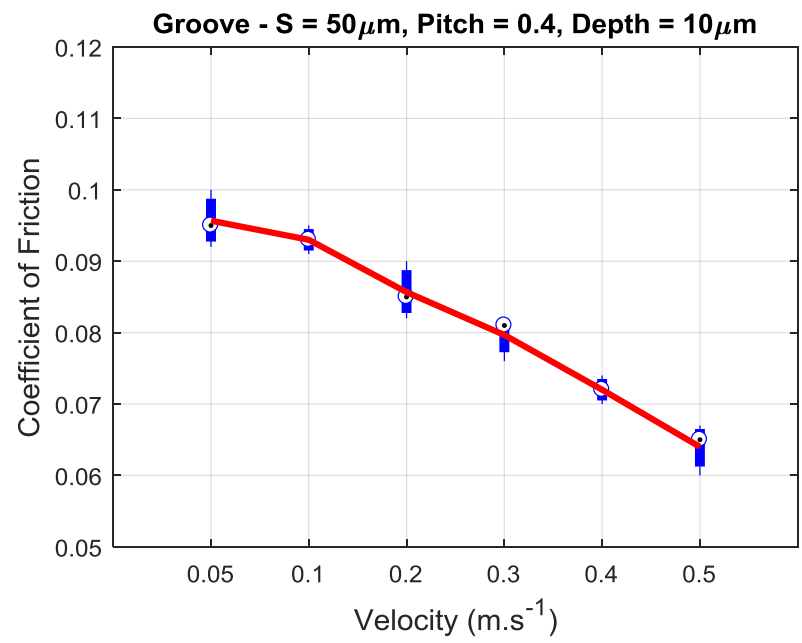

(b)

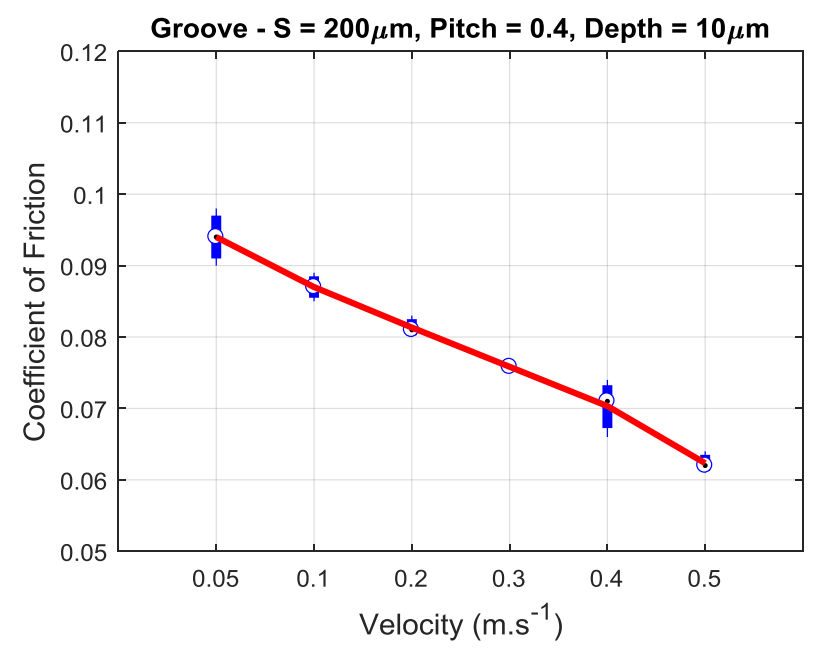

(c) 


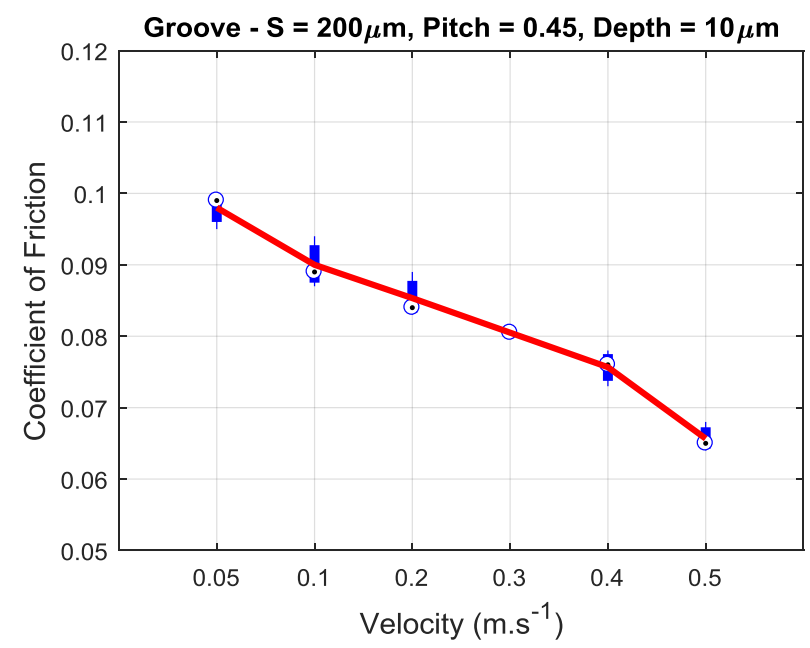

(d)

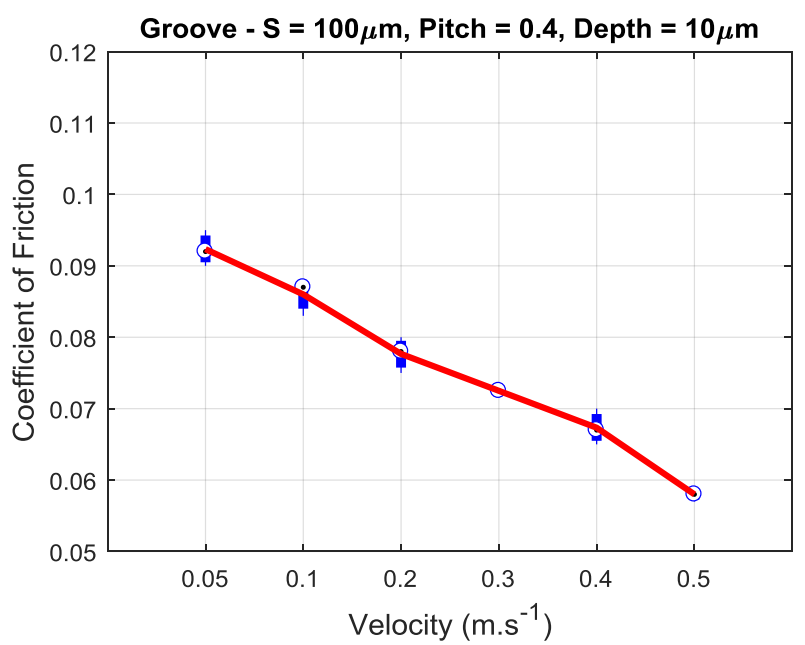

(e)

Figure 9. Coefficient of friction measurement results for grooved textured samples $\left(F_{T}=10 \mathrm{~N}\right.$ and $\eta=32 \mathrm{mPa}$. $\left.\mathrm{s}\right)$.

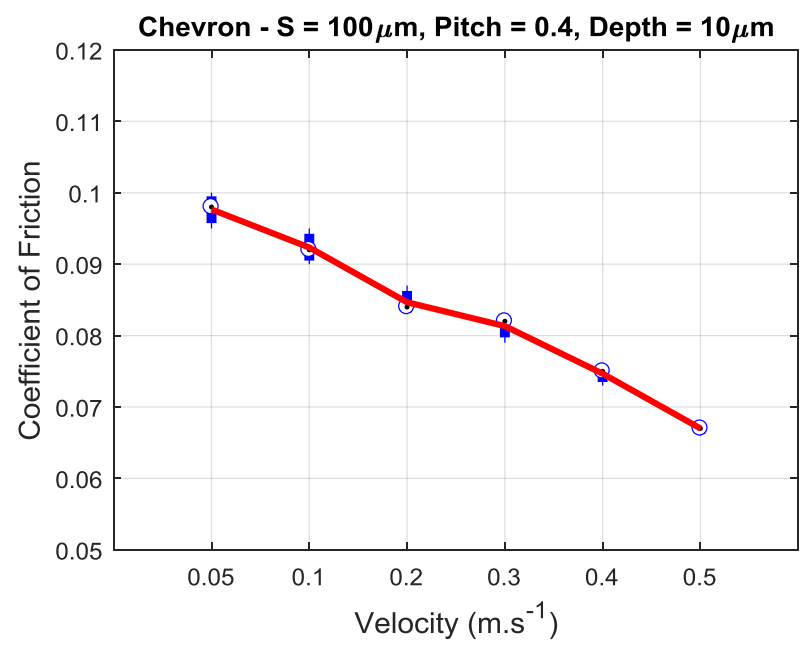

Figure 10. Coefficient of friction measurement results for chevron textured samples $\left(F_{T}=10 \mathrm{~N}\right.$ and $\eta=32 \mathrm{mPa}$. $\left.\mathrm{s}\right)$.
The figures show that the coefficient of friction in the boundary lubrication regime is around 0.10 , therefore in the subsections 3.3 to 3.6 where the calculated coefficient of friction and measured coefficient of friction are compared, this value for the BL coefficient of friction is used as input for the friction model.

\subsection{Pattern}

In this sub-section, the calculated coefficient of friction based on the numerical model is compared with the coefficient of friction measured with the pin-on-disk experiments. In Fig. 11, the numerical and experimental results of the coefficient of friction in the case of groove and chevron patterns are plotted. In this case the grooves have $10 \mu \mathrm{m}$ depth $\left(T_{d}\right), 100 \mu \mathrm{m}$ size $(S)$ and the pitch $\left(P_{x}\right)$ is equal to 0.4 . The size of the chevrons used in this experiment is equal to aforementioned value, i.e. $S=100 \mu m, T_{d}=10 \mu m$ and $P_{x}=0.4$.

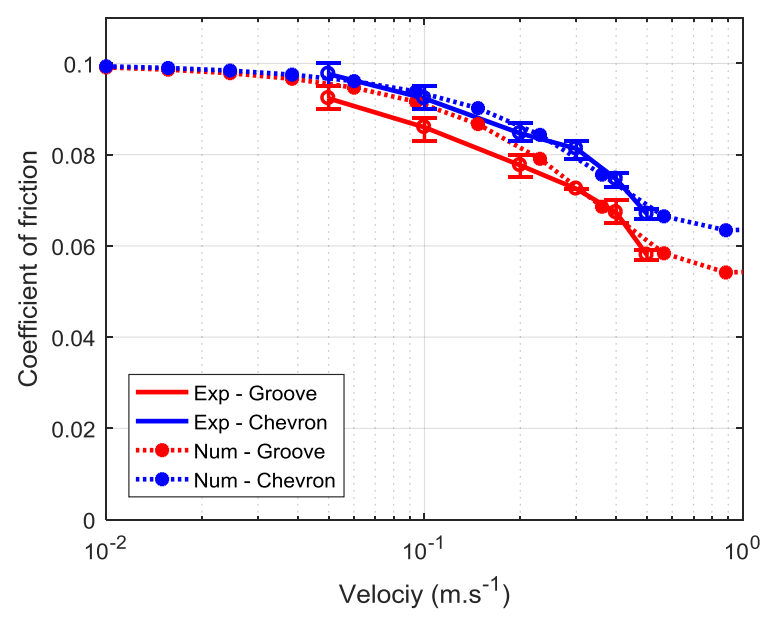

Figure 11. Numerical results vs experimental measurement results for groove and chevron patterns.

One can conclude from Fig 11 that both the numerical and the experimental coefficient of friction show the same trend for the coefficient of friction as a function of velocity (Stribeck curve). The calculated coefficient of friction is in close agreement with the measured coefficient of friction, and finally, both the numerical and experimental results show that the grooves are more effective than the chevrons for reducing the coefficient of friction. In both cases, the model is overestimating the measured coefficient of friction.

\subsection{Depth $\left(\mathbf{T}_{d}\right)$}

After checking the efficiency of the texture pattern, the influence of cavity depth on the frictional behaviour of 
the contacts was investigated. To achieve this goal, the coefficient of friction for two samples with a groove pattern is measured. The cavity depth for these two samples is respectively $5 \mu \mathrm{m}$ and $10 \mu \mathrm{m}$, the size parameter $(S)$ for both samples is equal to $100 \mu \mathrm{m}$ and the pitch parameter is equal to 0.4 . The measurements and calculations were based on the velocity range between $0.05 \mathrm{~m} . \mathrm{s}^{-1}$ and $0.5 \mathrm{~m} . \mathrm{s}^{-1}$. The numerical results and the experimental results are presented in Fig. 12.

In Fig. 12 the numerical results show that when the depth increases from $5 \mu \mathrm{m}$ to $10 \mu \mathrm{m}$, the coefficient of friction reduces and the trend in friction reduction is similar in both cases. A lower friction is achievable when the depth of the grooves is greater, due to a thicker film that is generated by applying grooves with a cavity depth close to the optimum value.

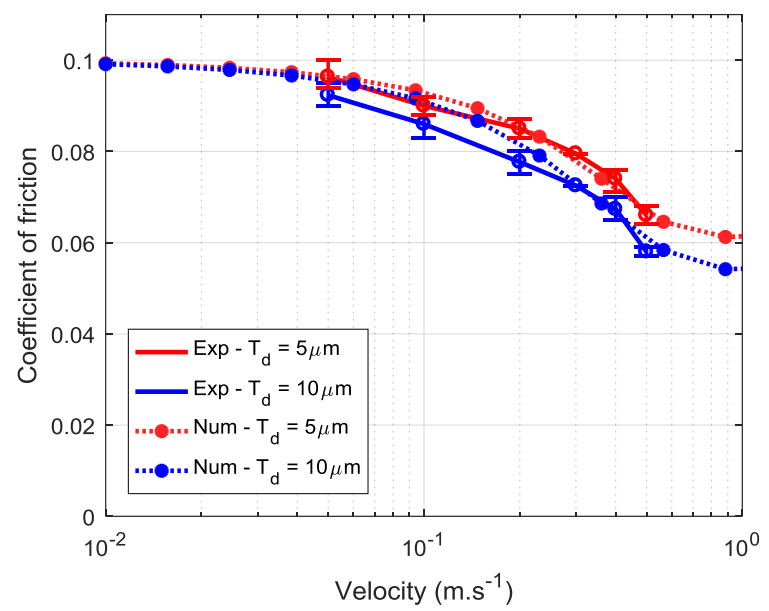

Figure 12. Comparison of numerical results vs experimental results for grooves with different cavity depth $\left(T_{d}\right)$.

\subsection{Size $(\boldsymbol{S})$}

These measurements were performed under $5 \mathrm{~N}$ normal load. The coefficient of friction for three samples with a groove pattern of a different size $(S)$ was measured. The cavity size for these three samples are $50 \mu \mathrm{m}, 100 \mu \mathrm{m}$ and $150 \mu \mathrm{m}$ respectively. The depth parameter $\left(T_{d}\right)$ for these samples is equal to $10 \mu \mathrm{m}$ and the pitch parameter $\left(P_{x}\right)$ is equal to 0.4 . The measurements and calculations were performed for a velocity range between $0.05 \mathrm{~m} . \mathrm{s}^{-1}$ to $0.5 \mathrm{~m} . \mathrm{s}^{-1}$. The numerical and experimental results are presented in Fig. 13.

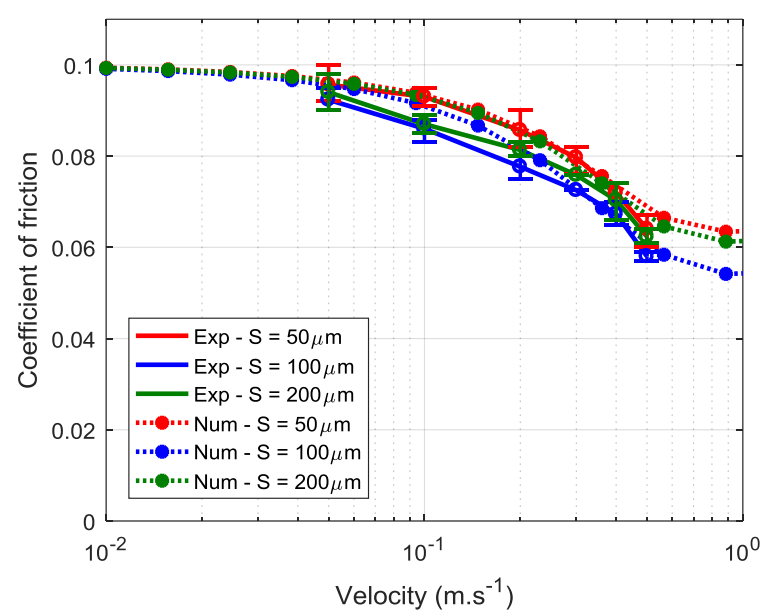

Figure 13. Comparison of numerical results vs experimental results for grooves with different cavity size (S).

Fig. 13, shows that, like the previous numericalexperimental comparison, the trend of friction reduction is similar in both cases and they are in the same range. These results validate that, when size of the grooves is around $100 \mu \mathrm{m}$ the lowest coefficient of friction is achievable, and the highest coefficient of friction for these three samples is obtained when the size of the cavities is around $50 \mu \mathrm{m}$. This frictional behaviour of groove pattern is due to the thicker film produced by applying the grooves with the cavity size close to the optimum value.

\subsection{Pitch $\left(\boldsymbol{P}_{x}\right)$}

The final parameter investigated is the pitch. The influence of this parameter on the frictional behaviour of sliding plane parallel contacts has been investigated experimentally and numerically. The coefficient of friction for two samples with a groove pattern is measured. The pitch parameters $\left(P_{x}\right)$ for these three samples are 0.40 and 0.45 , the depth parameter $\left(T_{d}\right)$ for these samples is equal to $10 \mu \mathrm{m}$ and the size $(S)$ for both of them is equal to $100 \mu \mathrm{m}$. The numerical as well as the experimental results are presented in Fig. 14. 


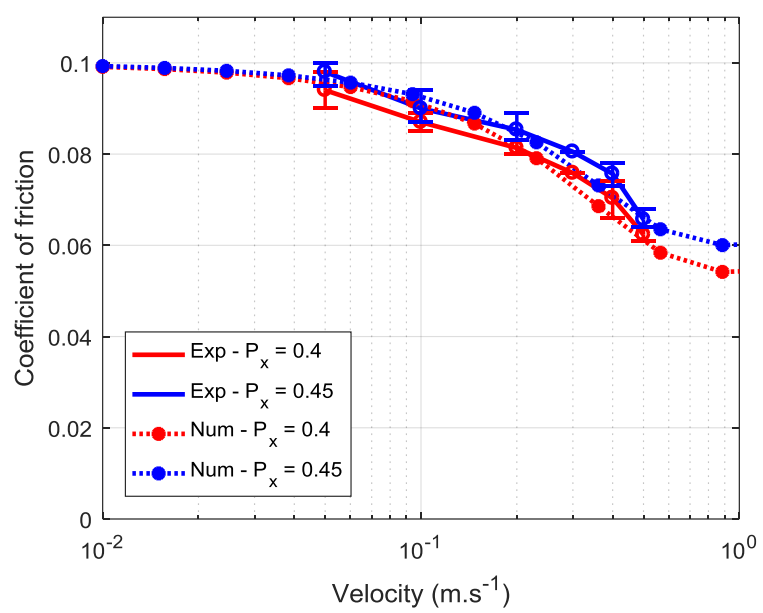

Figure 14. Comparison of numerical results vs experimental results for grooves with different pitch parameters $\left(P_{x}\right)$.

Figure 14 , shows that when $P_{x}$ is equal to 0.4 a lower coefficient of friction is obtained.

In section 3.3, the experimental results show that the grooves are more effective than the chevrons in reducing the coefficient of friction, which shows the dominant effect of more textured area than that created by applying chevrons. This effect is also observable from the numerical calculation.

\section{Conclusions}

In this paper, several experiments in order to validate the mixed lubrication model with respect to texturing parameters are performed. Firstly, measurements are performed to obtain the coefficient of friction under boundary lubrication conditions by using a nontextured sample. After that, measurements are performed to study the influence of different texturing parameters on friction and compared with simulations based on the model presented in this thesis. In conclusion:

- The trend of friction reduction both numerically and experimentally is similar in both cases.

- The calculated coefficient of friction is in close agreement with the measured coefficient of friction.

- The grooves are more effective in reducing the coefficient of friction both numerically and experimentally.

- The lowest friction is achievable when the depth of the grooves is around $10 \mu \mathrm{m}$.

- These results validate that when the size of grooves is around $100 \mu \mathrm{m}$, the lowest coefficient of friction is achievable.
- In the case of a groove pattern the lowest coefficient of friction is achievable when $P_{x}=$ 0.4 .

\section{Appendix A}

In numerical modeling of tribological phenomenon and to study the asperity contact, most of the contact mechanics theories like Greenwood and Williamson's contact model [14] (G\&W) approximate asperities geometry by assuming them as spheres or ellipsoids. Further, in G\&W the asperities are assumed as the spheres with the same radius and the summit heights is restricted to a Gaussian distribution but, in reality the asperities do not have the same radius and most of the surfaces have a height distribution, which is not Gaussian. In this study, the deterministic roughness model is used to calculate the load carried by the asperities of the measured surface when it is deformed by a flat surface. In the deterministic roughness model the digital data of the measured surface topography is provided by an interference microscope. In Fig. A1, the flat surface on a rough surface, contact is presented. In a deterministic model, by summing up the local contributions for any specific value of separation parameter $(d)$, it is possible to calculate several frictional parameters, such as the number of asperities, the real contact area and the total force carried by the contacts.

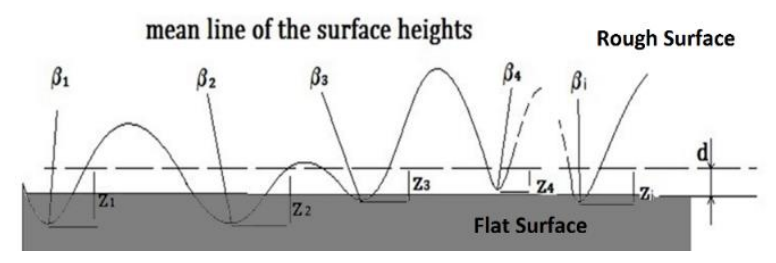

Figure A1. The contact between a rough surface and a flat surface [15].

In this model the asperities are assumed as ellipsoids with different radii which they deform independently from each other. The real contact area and normal load over the asperities are calculable based on the indentation and individual components of each asperity contact and it is possible to calculate the indentation of each asperity based on the given separation parameter and its individual summit height:

$$
w_{i}=z_{i}-d
$$


In the mixed lubrication regime the total normal load is achievable by knowing the normal load on asperity contact and fluid film [16]:

$$
F_{\mathrm{T}}=F_{\mathrm{C}}+F_{\mathrm{H}}
$$

Where $F_{T}$ is the total normal load, $F_{C}$ is the load carried by the asperities or boundary lubrication force component $(\mathrm{BL})$ and $F_{H}$ is the hydrodynamic lubrication ( $\mathrm{HL}$ ) force component, on (Eq. A.2), coefficients $\left(\gamma_{1}\right)$ and $\left(\gamma_{2}\right)$ are introduced:

$$
\gamma_{1}=\frac{F_{\mathrm{T}}}{F_{\mathrm{H}}}, \gamma_{2}=\frac{F_{\mathrm{T}}}{F_{\mathrm{C}}}
$$

These two coefficients $\left(\gamma_{1}\right.$ and $\left.\gamma_{2}\right)$ are mutually dependent:

$$
1=\frac{1}{\gamma_{1}}+\frac{1}{\gamma_{2}}
$$

In order to model the frictional behaviour of contacts in mixed lubrication three different equations must be solved, and the film thickness equation is the first equation to calculate the hydrodynamic component of mixed lubrication. In this study, the film thickness calculation is based on solving the Reynolds equation [17].

The Reynolds equation is deduced from the NavierStokes equations under the narrow gap and small Reynolds number assumptions. In addition, in film thickness calculation when the negative pressure is not negligible the cavitation effect must be considered. In this study the Jakobsson-Floberg-Olsson (JFO) model is employed to enhance the Reynolds equation in such a way as to consider the effect of cavitation in lubricant film. In (JFO) the lubrication film is divided into two zones.

- The zone with a complete lubricant film, in this region the Reynolds equation applies.

- The zone which the vapour bubbles are formed within the void fraction. In this zone, cavitation takes place and only a fraction of the lubricant film gap is occupied. The pressure throughout the cavitation area is taken as constant and is equal to cavitation pressure $[17,18]$.

According to the Payvar-Salant, taking cavitation into account the steady-state mass-conservation Reynolds equation can be written in a Cartesian coordinate system as (Eq. A.5) model [17, 19-21]:

$$
\begin{aligned}
& \frac{\partial}{\partial x}\left(\frac{h^{3}}{\eta} \frac{\partial(F \varphi)}{\partial x}\right)+\frac{\partial}{\partial x}\left(\frac{h^{3}}{\eta} \frac{\partial(F \varphi)}{\partial y}\right) \\
= & \frac{6 u_{0}}{p_{a}-p_{c}} \frac{\partial((1+(1-F) \varphi) h)}{\partial x}
\end{aligned}
$$

where $h$ is the film thickness, $\eta$ is the viscosity, $u_{0}$ is the sum velocity, $p_{a}$ is the ambient pressure, $p_{c}$ is the cavitation pressure, $F$ is a dimensionless dependent variable and $\varphi$ is the cavitation index $[17,21]$.

The second equation to calculate the friction of textured surfaces in mixed lubrication is the topography equation of textured area (Eq. A.6). The general film thickness formula can be written as (Eq. A.6):

$$
h=h_{0}+h_{\text {macro }}+h_{\text {texture }}
$$

In case of a flat-flat contact, the macro geometry is omitted and the film thickness (Eq. A.6) reduces to (Eq. A.7) $[22,23]$ :

$$
\frac{h(x, y)}{h_{0}(x, y)}=1+H(x, y)
$$

To solve (Eq. A.5) iteratively, the tri-diagonal matrix algorithm (TMDA) is used and for reducing the storage needed for calculation the line-by-line TDMA solver (Patankar [24]) is applied. For a two-dimensional problem, the TDMA solution column by column or row by row becomes iterative, and sweeping is done line by line and column by column or row by row. For threedimensional problems, the TDMA is applied line by line on a selected plane and then the calculation is moved to the next plane, scanning the domain plane-by-plane [25].

The third equation is derived from the equilibrium of the modified relation for the central pressure and average contact pressure carried by asperities, which it can be presented as [15].

$$
F_{c}=\sum_{i=1}^{N} \frac{2}{3} E^{\prime} \beta_{i}\left(z_{i}-d\right)
$$

By combining the shear force of the lubricant $\left(F_{f h}\right)$ and the friction force of the contacting asperities, the total friction force $\left(F_{f}\right)$ is achievable:

$$
F_{f}=\sum_{i=1}^{N} \iint_{A_{c i}} \tau_{c i} d A_{c i}+F_{f H}
$$

where the first term of right side of equation is the contacting asperity friction force and second term is the friction due to shear in the lubricant. In contacting asperity friction force term, $N$ is the number of contacting asperities, $\tau_{c i}$ is the shear stress at the asperity contact and $A_{c i}$ is the area of contact of a single asperity. By assuming the Coulomb type friction force for the contacting asperities, the relation between the average contact pressure $\left(p_{C i}\right)$ and the asperity coefficient of friction $\left(\mu_{C i}\right)$ is: 


$$
\mu_{c i}=\frac{\tau_{C i}}{p_{C i}}
$$

The asperity contact coefficient of friction $\left(\mu_{C i}\right)$ is assumed constant for all asperities, therefore the boundary lubrication component of the Eq. A. 8 can be written as:

$$
\sum_{i=1}^{N} \iint_{\mathrm{A}_{\mathrm{ci}}} \mu_{c} P_{c i} d A_{c i}=\mu_{c} F_{C}
$$

In this study, this value the value of $\mu_{C}$ is determined experimentally and is extracted from tribometer measurements. The coefficient of friction can be written as:

$$
\mu=\frac{F_{f}}{F_{N}}=\frac{\mu_{c} F_{C}+F_{f H}}{F_{N}}
$$

In order to study the effect surface and to compare the measurements results with the numerical calculations several simulations are carried out. The samples' characteristics for these calculations are presented in table A.1.

Table A.1. Samples characteristics.

\begin{tabular}{cc}
\hline Parameter & Value \\
\hline Elasticity modulus, $E$ & $210 \mathrm{GPa}$ \\
Poisson's ratio, $v$ & 0.3 \\
\hline
\end{tabular}

By using the measured data for the surface roughness, the 3D illustration is calculated (see Figure A2).

The coefficient of friction in the ML regime for the starved condition can be calculated as explained in the chart presented below, (see figure A.3). 


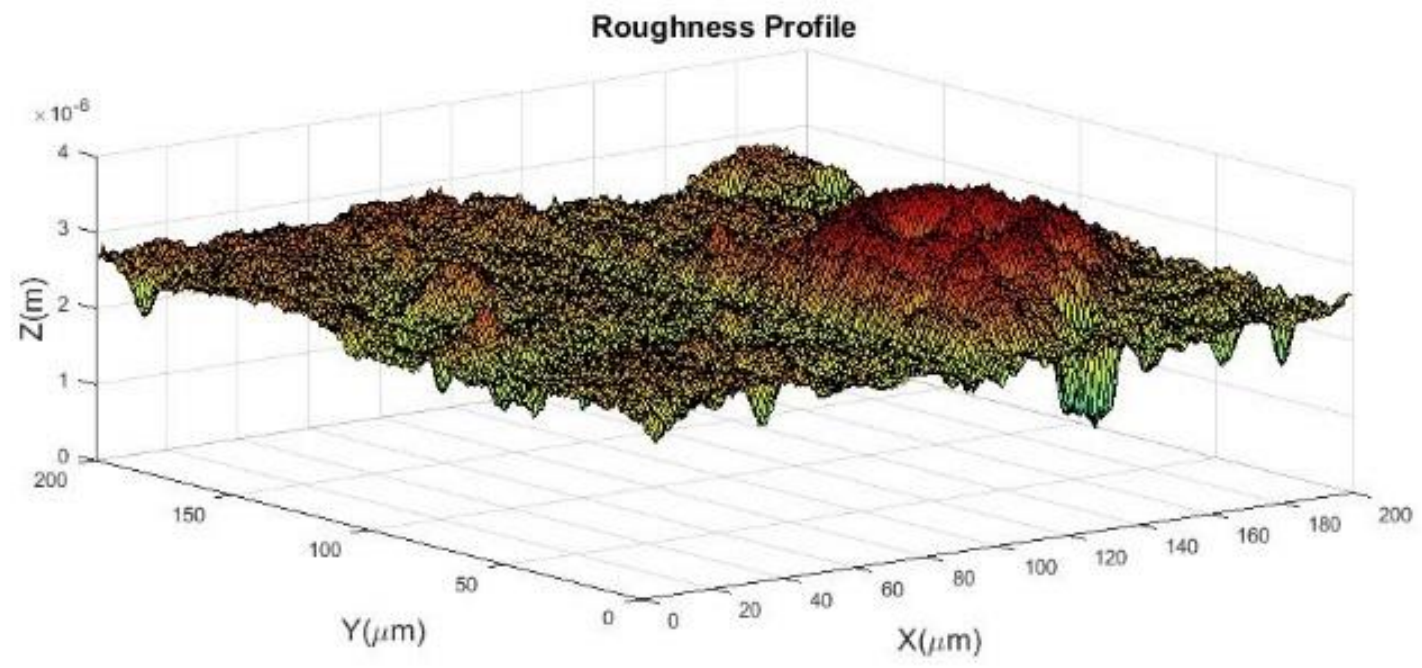

Figure A.2. Roughness measurement surface profile based on the measured roughness by Laser Scanning Microscope measurements, in this case $R_{a}=0.11 \mu \mathrm{m}$.

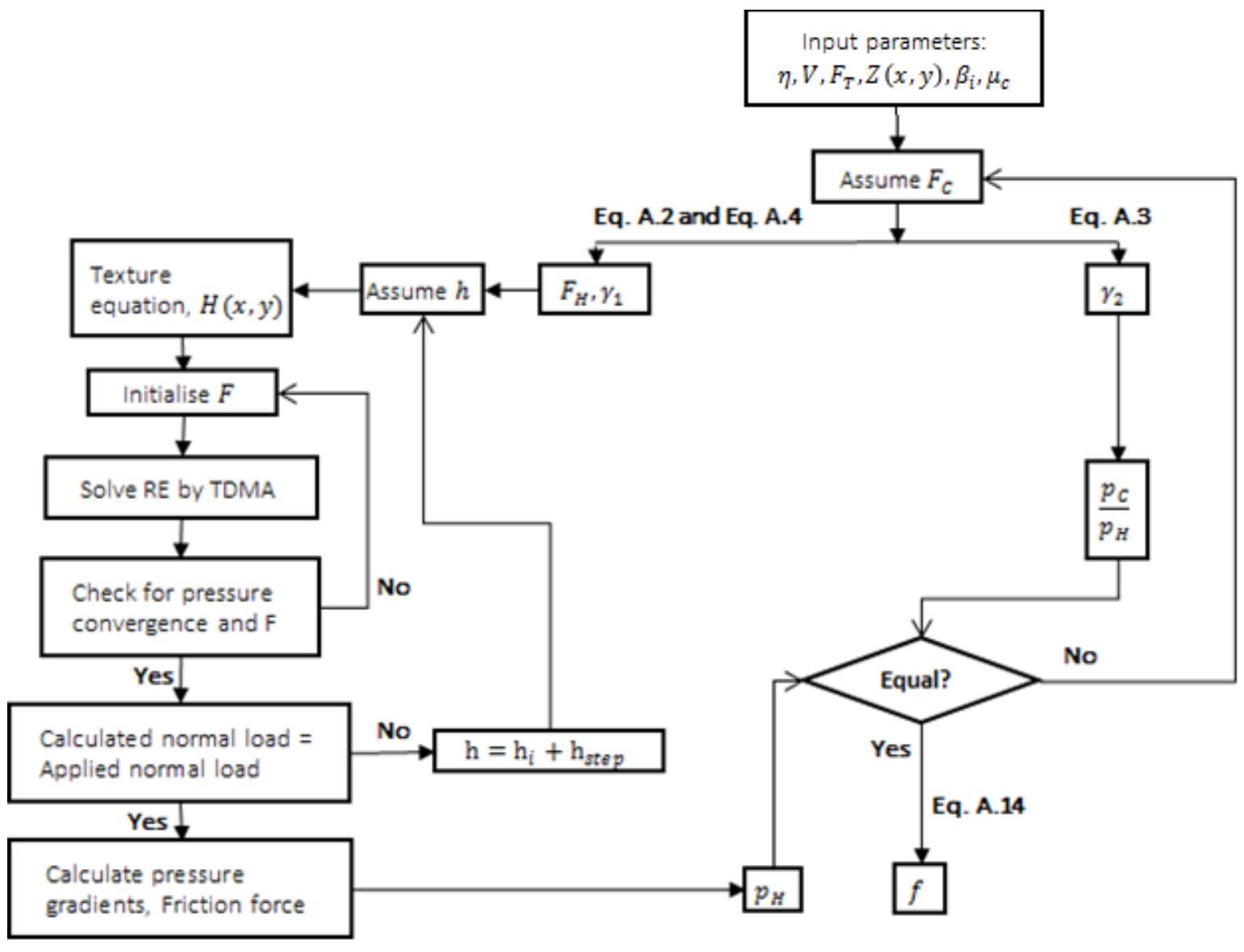

Figure A.3. Mixed lubrication model. 


\section{Nomenclature}

\begin{tabular}{|c|c|c|}
\hline Parameters & Description & Unit \\
\hline$E^{\prime}$ & Reduced elasticity modulus & $P a$ \\
\hline$F$ & $\begin{array}{l}\text { Elrod cavitation algorithm } \\
\text { switch function }\end{array}$ & - \\
\hline$F_{C}$ & Load carried by the asperities & $N$ \\
\hline$F_{f}$ & Friction force & $N$ \\
\hline$F_{f H}$ & Hydrodynamic friction force & $N$ \\
\hline$F_{H}$ & $\begin{array}{l}\text { Load carried by the } \\
\text { hydrodynamic component }\end{array}$ & $N$ \\
\hline$F_{N}$ & $\begin{array}{l}\text { Dimensionless total normal } \\
\text { load }\end{array}$ & - \\
\hline$F_{T}$ & Normal load on the contact & $N$ \\
\hline$f$ & Coefficient of friction & - \\
\hline$f_{C}$ & $\begin{array}{l}\text { Coefficient of friction in } \mathrm{BL} \\
\text { regime }\end{array}$ & - \\
\hline$H$ & $\begin{array}{l}\text { Dimensionless local depth of } \\
\text { textured surface }\end{array}$ & - \\
\hline$h_{0}$ & Contact separation & $m$ \\
\hline$L_{g x}$ & $\begin{array}{l}\text { Texture cell length in } \mathrm{x}- \\
\text { direction }\end{array}$ & $m$ \\
\hline$L_{g y}$ & $\begin{array}{l}\text { Texture cell length in } y \text { - } \\
\text { direction }\end{array}$ & $m$ \\
\hline$L_{x}$ & Textured area in $\mathrm{x}$-direction & $m$ \\
\hline$L_{y}$ & Textured area in $y$-direction & $m$ \\
\hline$P$ & Dimensionless pressure & - \\
\hline$P_{x}$ & Texture pitch & - \\
\hline$p$ & Pressure & $\mathrm{Pa}$ \\
\hline$p_{a}$ & Ambient pressure & $\mathrm{Pa}$ \\
\hline$r$ & $\begin{array}{l}\text { Texture cell length in } \mathrm{x} \& \mathrm{y}- \\
\text { direction, in the case of } \\
\text { dimples }\end{array}$ & $m$ \\
\hline$r_{p}$ & Cavity characteristic width & $m$ \\
\hline$U_{0}$ & Sum velocity & $m \cdot s^{-1}$ \\
\hline$u_{0}$ & Sum velocity & $m \cdot s^{-1}$ \\
\hline$S$ & Cavity size $=2 r_{p}$ & $m$ \\
\hline$T_{d}$ & Texture depth & $m$ \\
\hline$w_{i}$ & Compliance of an asperity & $m$ \\
\hline$z_{i}$ & Asperity height & $m$ \\
\hline$G$ & Geometric parameter & - \\
\hline$\beta_{i}$ & Combined radius of summits & - \\
\hline$\eta$ & Dynamic viscosity & Pa.s \\
\hline$\mu_{c}$ & $\begin{array}{l}\text { Coefficient of friction in } \mathrm{BL} \\
\text { regime }\end{array}$ & - \\
\hline$\rho$ & Density & $\mathrm{kg} \cdot \mathrm{m}^{3}$ \\
\hline$\rho_{c}$ & $\begin{array}{l}\text { Lubricant density in full film } \\
\text { region }\end{array}$ & $\mathrm{kg} \cdot \mathrm{m}^{3}$ \\
\hline$\varphi$ & $\begin{array}{l}\text { Cavitation dimensionless } \\
\text { variable }\end{array}$ & - \\
\hline
\end{tabular}

\section{References}

[1] Glavatskih, S. B., McCarthy, D. M. C., and Sherrington, I., 2005, "Hydrodynamic Performance of a Thrust Bearing with Micropatterned Pads," Tribology Transactions, 48(4), pp. 492-498.

[2] Brizmer, V., Kligerman, Y., and Etsion, I., 2003, "A Laser Surface Textured Parallel Thrust Bearing," Tribology Transactions, 46(3), pp. 397-403.

[3] Etsion, I., 2010, "State of the Art in Laser Surface Texturing," Advanced Tribology, J. Luo, Y. Meng, T. Shao, and Q. Zhao, eds., Springer Berlin Heidelberg, pp. 761-762.

[4] Etsion, I., and Burstein, L., 1996, "A Model for Mechanical Seals with Regular Microsurface Structure," Tribology Transactions, 39(3), pp. 677 683.

[5] Etsion, I., Halperin, G., Brizmer, V., and Kligerman, Y., 2004, "Experimental Investigation of Laser Surface Textured Parallel Thrust Bearings," Tribology Letters, 17(2), pp. 295-300.

[6] Etsion, I., Kligerman, Y., and Halperin, G., 1999, "Analytical and Experimental Investigation of Laser-Textured Mechanical Seal Faces," Tribology Transactions, 42(3), pp. 511-516.

[7] Kovalchenko, A., Ajayi, O., Erdemir, A., Fenske, G., and Etsion, I., 2005, "The effect of laser surface texturing on transitions in lubrication regimes during unidirectional sliding contact," Tribology International, 38(3), pp. 219-225.

[8] Ronen, A., Etsion, I., and Kligerman, Y., 2001, "Friction-Reducing Surface-Texturing in Reciprocating Automotive Components," Tribology Transactions, 44(3), pp. 359-366.

[9] Ryk, G., Kligerman, Y., and Etsion, I., 2002, "Experimental Investigation of Laser Surface Texturing for Reciprocating Automotive Components," Tribology Transactions, 45(4), pp. 444-449.

[10] Vilhena, L. M., Podgornik, B., Vižintin, J., and Možina, J., 2011, "Influence of texturing parameters and contact conditions on tribological behaviour of laser textured surfaces," Meccanica, 46(3), pp. 567-575.

[11] Galda, L., Pawlus, P., and Sep, J., 2009, "Dimples shape and distribution effect on characteristics of Stribeck curve," Tribology International, 42(10), pp. 1505-1512.

[12] Galda, L., Sep, J., Olszewski, A., and Zochowski, T., 2019, "Experimental investigation into surface texture effect on journal bearings performance," Tribology International.

[13] Zhong, Y., Zheng, L., Gao, Y., and Liu, Z., 2019, "Numerical simulation and experimental investigation of tribological performance on 
bionic hexagonal textured surface," Tribology International, 129, pp. 151-161.

[14] Qiu, M., Minson, B. R., and Raeymaekers, B., 2013, "The effect of texture shape on the friction coefficient and stiffness of gas-lubricated parallel slider bearings," Tribology International, 67, pp. 278-288.

[15] Greenwood, J. A., and Williamson, J. B. P., 1966, "Contact of Nominally Flat Surfaces," Proceedings of the Royal Society of London. Series A. Mathematical and Physical Sciences, 295(1442), pp. 300-319.

[16] Faraon, I. C., 2005, "Mixed lubricated line contacts, PhD thesis,"PhD Thesis, University of Twente, Enschede.

[17] Johnson, K. L., Greenwood, J. A., and Poon, S. Y., 1972, "A simple theory of asperity contact in elastohydro-dynamic lubrication," Wear, 19(1), pp. 91-108.

[18] Bijani, D., Deladi, L. E., and Schipper, D. J., 2016, "The Influence of Surface Texturing on the Film Thickness in Parallel Sliding Surfaces," 20th International Colloquium Tribology: Technische Akademie Esslingen, p. 12.
[19] Coyne, J. C., and Elrod, H. G., 1965, Conditions for the Rupture of a Lubrication Film.

[20] Braun, M. J., and Hannon, W. M., 2010, "Cavitation formation and modelling for fluid film bearings: A review," Proceedings of the Institution of Mechanical Engineers, Part J: Journal of Engineering Tribology, 224(9), pp. 839-863.

[21] Payvar, P., and Salant, R. F., 1992, "Computational method for cavitation in a wavy mechanical seal," Journal of Tribology, 114(1), pp. 199-204.

[22] Xiong, S., and Wang, Q. J., 2012, "Steady-state hydrodynamic lubrication modeled with the payvar-salant mass conservation model," Journal of Tribology, 134(3).

[23] Qiu, M., Delic, A., and Raeymaekers, B., 2012, "The effect of texture shape on the load-carrying capacity of gas-lubricated parallel slider bearings," Tribology Letters, 48(3), pp. 315-327.

[24] Patankar, S. V., 1980, Numerical heat transfer and fluid flow / Suhas V. Patankar, Hemisphere Pub. Corp. ; McGraw-Hill, Washington : New York.

[25] Versteeg, H. K., and Malalasekera, W., 1995, An Introduction to Computational Fluid Dynamics. The Finite Volume Method, Longman Group Ltd. 